\title{
OPTIMIZACIÓN MULTIOBJETIVO INTELIGENTE DEL DIMENSIONAMIENTO ENERGÉTICO EN SISTEMAS FERROVIARIOS SEÑALIZADOS Y EN PLATAFORMAS SOFTWARE DE TIEMPO REAL
}

Autor: Manuel Soler Nicolau

Director: José Manuel Mera Sánchez de Pedro 


\section{Resumen}

La capacidad de transporte es uno de los baremos fundamentales para evaluar la progresión que puede llegar a tener un área económica y social. Es un sector de elevada importancia para la sociedad actual. Englobado en los distintos tipos de transporte, uno de los medios de transporte que se encuentra más en alza en la actualidad, es el ferroviario. Tanto para movilidad de pasajeros como para mercancías, el tren se ha convertido en un medio de transporte muy útil. Se encuentra dentro de las ciudades, entre ciudades con un radio pequeño entre ellas e incluso cada vez más, gracias a la alta velocidad, entre ciudades con gran distancia entre ellas. Esta Tesis pretende ayudar en el diseño de una de las etapas más importantes de los Proyectos de instalación de un sistema ferroviario: el sistema eléctrico de tracción.

La fase de diseño de un sistema eléctrico de tracción ferroviaria se enfrenta a muchas dudas que deben ser resueltas con precisión. Del éxito de esta fase dependerá la capacidad de afrontar las demandas de energía de la explotación ferroviaria. También se debe atender a los costes de instalación y de operación, tanto costes directos como indirectos. Con la Metodología que se presenta en esta Tesis se ofrecerá al diseñador la opción de manejar un sistema experto que como soluciones le plantee un conjunto de escenarios de sistemas eléctricos correctos, comprobados por resolución de modelos de ecuaciones. Correctos desde el punto de vista de validez de distintos parámetros eléctrico, como de costes presupuestarios e impacto de costes indirectos. Por tanto, el diseñador al haber hecho uso de esta Metodología, tendría en un espacio de tiempo relativamente corto, un conjunto de soluciones factibles con las que poder elegir cuál convendría más según sus intereses finales.

Esta Tesis se ha desarrollado en una vía de investigación integrada dentro del Centro de Investigaciones Ferroviarias CITEF-UPM. Entre otros proyectos y vías de investigación, en CITEF se ha venido trabajando en estudios de validación y dimensionamiento de sistemas eléctricos ferroviarios con diversos y variados clientes y sistemas ferroviarios. A lo largo de los proyectos realizados, el interés siempre ha girado mayoritariamente sobre los siguientes parámetros del sistema eléctrico:

- Calcular número y posición de subestaciones de tracción. Potencia de cada subestación.

- Tipo de catenaria a lo largo del recorrido. Conductores que componen la catenaria. Características.

- Calcular número y posición de autotransformadores para sistemas funcionando en alterna bitensión o $2 \mathrm{x} 25 \mathrm{kV}$.

- Posición Zonas Neutras.

- Validación según normativa de:

- Caídas de tensión en la línea

- Tensiones máximas en el retorno de la línea

- Sobrecalentamiento de conductores 
- Sobrecalentamiento de los transformadores de las subestaciones de tracción

La idea es que las soluciones aportadas por la Metodología sugieran escenarios donde de estos parámetros estén dentro de los límites que marca la normativa. Tener la posibilidad de tener un repositorio de posibles escenarios donde los parámetros y elementos eléctricos estén calculados como correctos, aporta un avance en tiempos y en pruebas, que mejoraría ostensiblemente el proceso habitual de diseño para los sistemas eléctricos ferroviarios.

Los costes directos referidos a elementos como subestaciones de tracción, autotransformadores, zonas neutras, ocupan un gran volumen dentro del presupuesto de un sistema ferroviario. En esta Tesis se ha querido profundizar también en el efecto de los costes indirectos provocados en la instalación y operación de sistemas eléctricos. Aquellos derivados del impacto medioambiental, los costes que se generan al mantener los equipos eléctricos y la instalación de la catenaria, los costes que implican la conexión entre las subestaciones de tracción con la red general o de distribución y por último, los costes de instalación propios de cada elemento compondrían los costes indirectos que, según experiencia, se han pensado relevantes para ejercer un cierto control sobre ellos. La Metodología cubrirá la posibilidad de que los diseños eléctricos propuestos tengan en cuenta variaciones de coste inasumibles o directamente, proponer en igualdad de condiciones de parámetros eléctricos, los más baratos en función de los costes comentados.

Analizando los costes directos e indirectos, se ha pensado dividir su impacto entre los que se computan en la instalación y los que suceden posteriormente, durante la operación de la línea ferroviaria. Estos costes normalmente suelen ser contrapuestos, cuánto mejor es uno peor suele ser el otro y viceversa, por lo que hace falta un sistema que trate ambos objetivos por separado.

Para conseguir los objetivos comentados, se ha construido la Metodología sobre tres pilares básicos:

- Simulador ferroviario Hamlet: Este simulador integra módulos para construir esquemas de vías ferroviarios completos; módulo de simulación mecánica y de la tracción de material rodante; módulo de señalización ferroviaria; módulo de sistema eléctrico. Software realizado en C++ y Matlab.

- Análisis y estudio de cómo focalizar los distintos posibles escenarios eléctricos, para que puedan ser examinados rápidamente. Pico de demanda máxima de potencia por el tráfico ferroviario.

- Algoritmos de optimización: A partir de un estudio de los posibles algoritmos adaptables a un sistema tan complejo como el que se plantea, se decidió que los algoritmos genéticos serían los elegidos. Se han escogido 3 algoritmos genéticos, permitiendo recabar información acerca del comportamiento y resultados de cada uno de ellos. Los elegidos por motivos de tiempos de 
respuesta, multiobjetividad, facilidad de adaptación y buena y amplia aplicación en proyectos de ingeniería fueron: NSGA-II, AMGA-II y $\varepsilon$-MOEA.

- Diseño de funciones y modelo preparado para trabajar con los costes directos e indirectos y las restricciones básicas que los escenarios eléctricos no deberían violar. Estas restricciones vigilan el comportamiento eléctrico y la estabilidad presupuestaria.

Las pruebas realizadas utilizando el sistema han tratado o bien de copiar situaciones que se puedan dar en la realidad o directamente sistemas y problemas reales. Esto ha proporcionado además de la posibilidad de validar la Metodología, también se ha posibilitado la comparación entre los algoritmos genéticos, comparar sistemas eléctricos escogidos con los reales y llegar a conclusiones muy satisfactorias.

La Metodología sugiere una vía de trabajo muy interesante, tanto por los resultados ya obtenidos como por las oportunidades que puede llegar a crear con la evolución de la misma. Esta Tesis se ha desarrollado con esta idea, por lo que se espera pueda servir como otro factor para trabajar con la validación y diseño de sistemas eléctricos ferroviarios.

\section{Abstract}

Transport capacity is one of the critical points to evaluate the progress than a specific social and economical area is able to reach. This is a sector of high significance for the actual society. Included inside the most common types of transport, one of the means of transport which is elevating its use nowadays is the railway. Such as for passenger transport of weight movements, the train is being consolidated like a very useful mean of transport. Railways are installed in many geography areas. Everyone know train in cities, or connecting cities inside a surrounding area or even more often, taking into account the high-speed, there are railways infrastructure between cities separated with a long distance. This Ph.D work aims to help in the process to design one of the most essential steps in Installation Projects belonging to a railway system: Power Supply System.

Design step of the railway power supply, usually confronts to several doubts and uncertainties, which must be solved with high accuracy. Capacity to supply power to the railway traffic depends on the success of this step. On the other hand is very important to manage the direct and indirect costs derived from Installation and Operation. With the Methodology is presented in this Thesis, it will be offered to the designer the possibility to handle an expert system that finally will fill a set of possible solutions. These solutions must be ready to work properly in the railway system, and they were tested using complex equation models.

This Thesis has been developed through a research way, integrated inside Citef (Railway Research Centre of Technical University of Madrid). Among other projects and research ways, in Citef has been working in several validation studies and dimensioning of railway power supplies. It is been working by a large range of clients 
and railways systems. Along the accomplished Projects, the main goal has been rounded mostly about the next list of parameters of the electrical system:

- Calculating number and location of traction substations. Power of each substation.

- Type of Overhead contact line or catenary through the railway line. The wires which set up the catenary. Main Characteristics.

- Calculating number and position of autotransformers for systems working in alternating current bi-voltage of called $2 \times 25 \mathrm{kV}$.

- Location of Neutral Zones.

- Validating upon regulation of:

○ Drop voltages along the line

- Maximum return voltages in the line

O Overheating/overcurrent of the wires of the catenary

- Avoiding overheating in the transformers of the traction substations.

Main objective is that the solutions given by the Methodology, could be suggest scenarios where all of these parameters from above, would be between the limits established in the regulation. Having the choice to achieve a repository of possible good scenarios, where the parameters and electrical elements will be assigned like ready to work, that gives a great advance in terms of times and avoiding several tests. All of this would improve evidently the regular railway electrical systems process design.

Direct costs referred to elements like traction substations, autotransformers, neutral zones, usually take up a great volume inside the general budget in railway systems. In this Thesis has been thought to bear in mind another kind of costs related to railway systems, also called indirect costs. These could be enveloped by those enmarked during installation and operation of electrical systems. Those derived from environmental impact; costs generated during the maintenance of the electrical elements and catenary; costs involved in the connection between traction substations and general electric grid; finally costs linked with the own installation of the whole electrical elements needed for the correct performance of the railway system. These are integrated inside the set has been collected taking into account own experience and research works. They are relevant to be controlled for our Methodology, just in case for the designers of this type of systems. The Methodology will cover the possibility that the final proposed power supply systems will be hold non-acceptable variations of costs, comparing with initial expected budgets, or directly assuming a threshold of budget for electrical elements in actual scenario, and achieving the cheapest in terms of commented costs from above.

Analyzing direct and indirect costs, has been thought to divide their impact between two main categories. First one will be inside the Installation and the other category will comply with the costs often happens during Railway Operation time. These costs normally are opposed, that means when one is better the other turn into worse, in costs meaning. For this reason is necessary treating both objectives separately, in order to evaluate correctly the impact of each one into the final system. 
The objectives detailed before build the Methodology under three basic pillars:

- Railway simulator Hamlet: This software has modules to configure many railway type of lines; mechanical and traction module to simulate the movement of rolling stock; signaling module; power supply module. This software has been developed using $\mathrm{C}++$ and Matlab R13a

- Previously has been mandatory to study how would be possible to work properly with a great number of feasible electrical systems. The target comprised the quick examination of these set of scenarios in terms of time. This point is talking about Maximum power demand peaks by railway operation plans.

- Optimization algorithms. A railway infrastructure is a very complex system. At the beginning it was necessary to search about techniques and optimization algorithms, which could be adaptable to this complex system. Finally three genetic multiobjective algorithms were the chosen. Final decision was taken attending to reasons such as time complexity, able to multiobjective, easy to integrate in our problem and with a large application in engineering tasks. They are: NSGA-II, AMGA-II and $\varepsilon$-MOEA.

- Designing objectives functions and equation model ready to work with the direct and indirect costs. The basic restrictions are not able to avoid, like budgetary or electrical, connected hardly with the recommended performance of elements, catenary and safety in a electrical railway systems.

The battery of tests launched to the Methodology has been designed to be as real as possible. In fact, due to our work in Citef and with real Projects, has been integrated and configured three real railway lines, in order to evaluate correctly the final results collected by the Methodology. Another topic of our tests has been the comparison between the performances of the three algorithms chosen. Final step has been the comparison again with different possible good solutions, it means power supply system designs, provided by the Methodology, testing the validity of them. Once this work has been finished, the conclusions have been very satisfactory.

Therefore this Thesis suggest a very interesting way of research and work, in terms of the results obtained and for the future opportunities can be created with the evolution of this. This Thesis has been developed with this idea in mind, so is expected this work could adhere another factor to work in the difficult task of validation and design of railway power supply systems. 


\section{Índice}

1. Introducción. Evolución sistemas de aporte de energía en el ferrocarril. Motivación y objetivos de la Tesis.....................................................................5

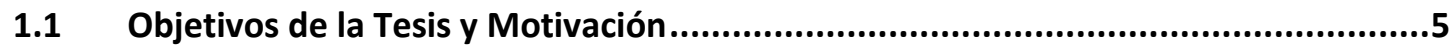

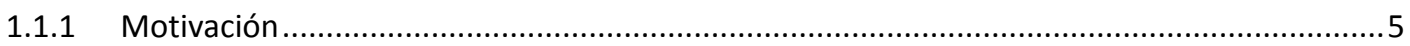

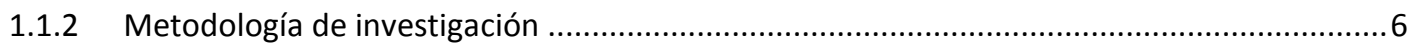

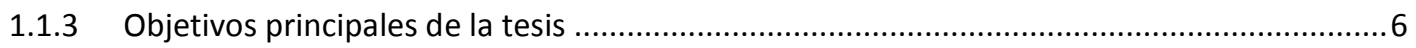

1.2 Sistemas de energía aplicados a líneas ferroviarias. Breve repaso histórico y

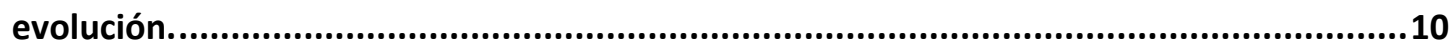

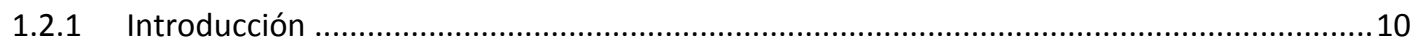

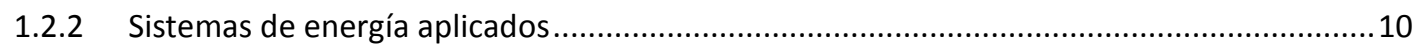

1.2.3 Implantación general del sistema de tracción eléctrico ferroviario e impacto social y

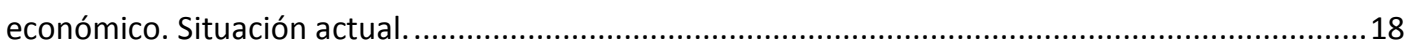

1.2.4 Características básicas Sistema eléctrico de tracción ferroviaria ..........................................20

2. Sistemas de electrificación Ferroviaria. Elementos clave para distribución de

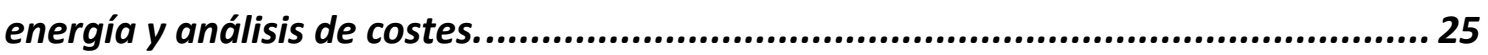

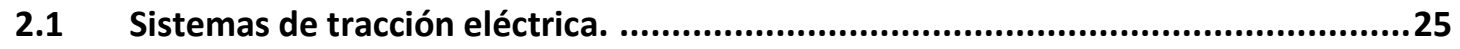

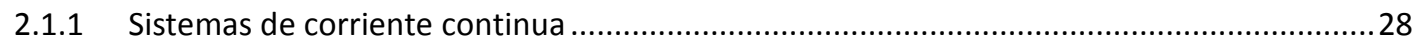

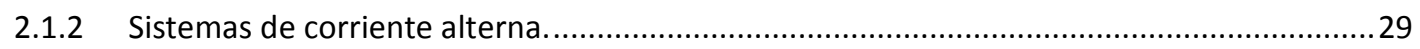

2.2 Elementos básicos sistemas electrificación ferroviaria. Instalación.......................37

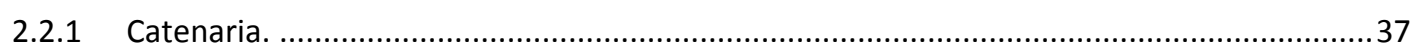

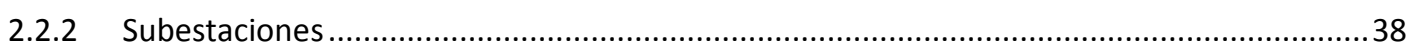

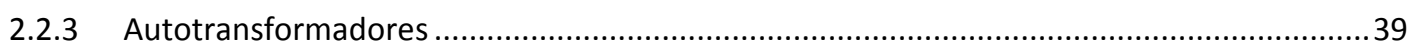

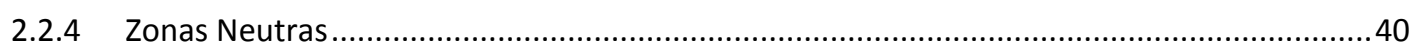

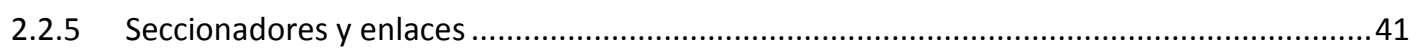

2.3 Zonas de especial interés para la evaluación de sistemas eléctricos ferroviarios ...42

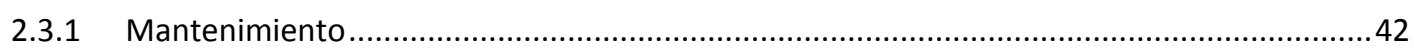

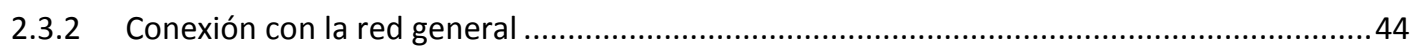

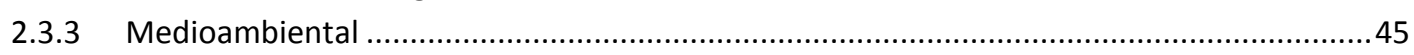

3. Herramienta software de simulación ferroviaria. Hamlet. ..............................50

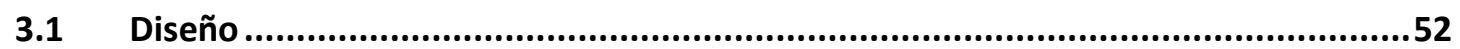

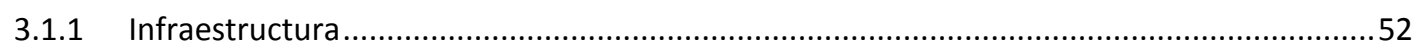

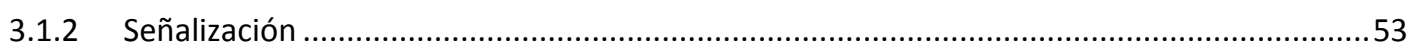

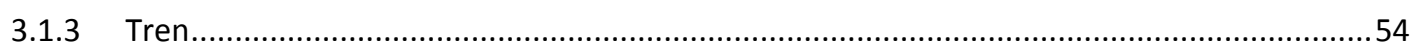

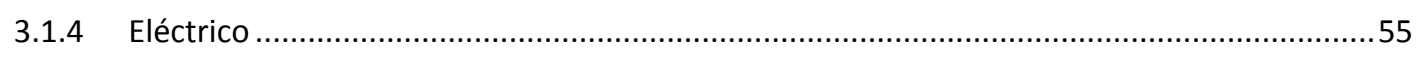

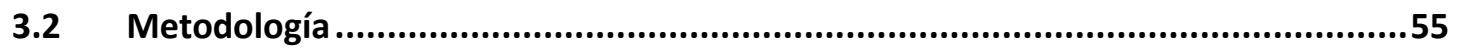

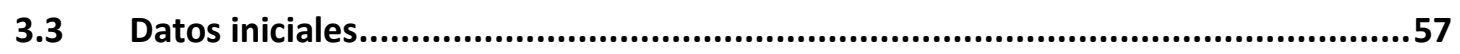

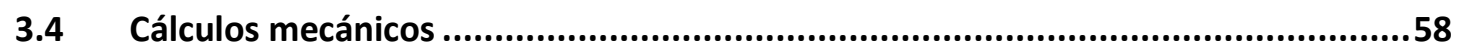

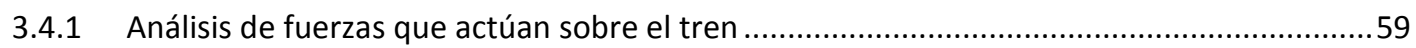

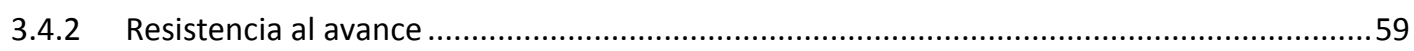




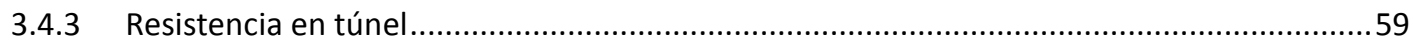

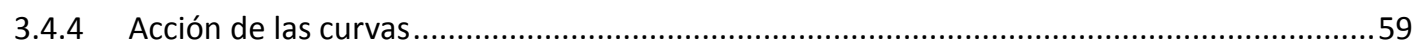

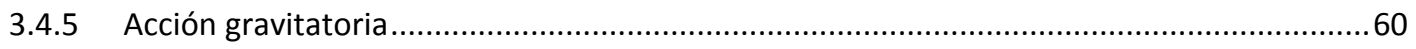

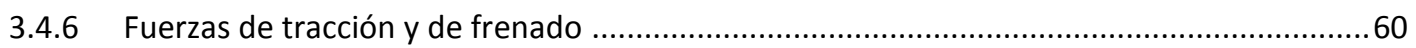

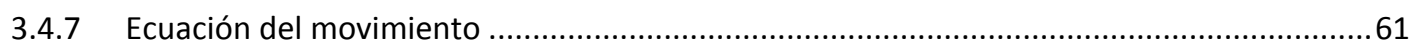

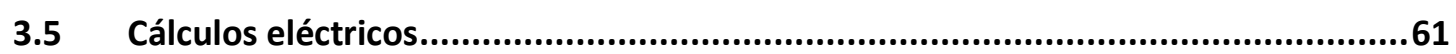

3.5.1 Modelo de la sección transversal .....................................................................................61

3.5.2 Calculo de la matriz de impedancias de los conductores ................................................62

3.5.3 Cálculo de la matriz de impedancias paralelo de los conductores ....................................63

3.5.4 Cálculo de las tensiones, intensidades y potencia en la línea ..........................................64

3.5.5 Cálculo de las intensidades de cortocircuito ................................................................67

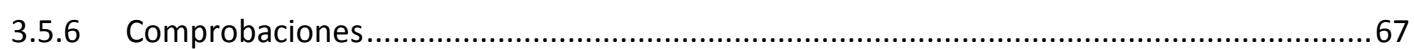

Resultados..................................................................................67

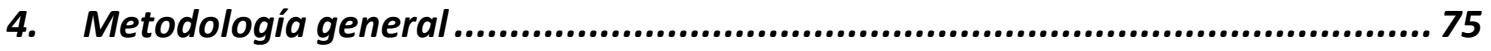

4.1 Algoritmos de optimización aplicados a problemas de Ingeniería .........................75

4.2 Elección de la Técnica de Optimización. Criterios clave y motivación. ...................884

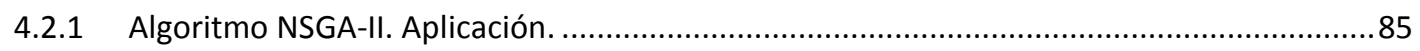

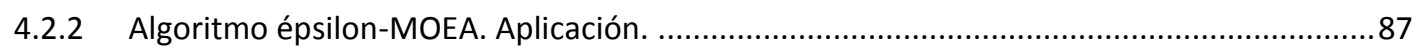

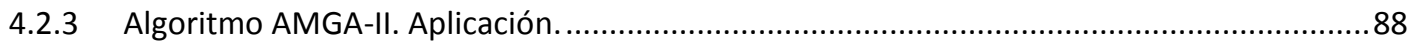

4.2.4 Aplicación de conjunto de algoritmos genéticos. Comparativa. ..........................................99

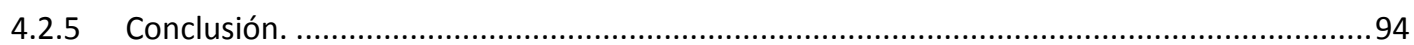

4.3 Discretización zonal y ponderación de costes.................................................95

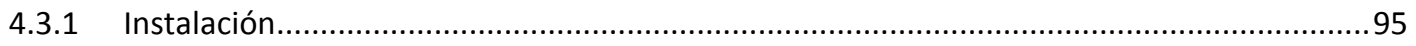

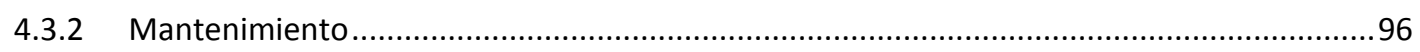

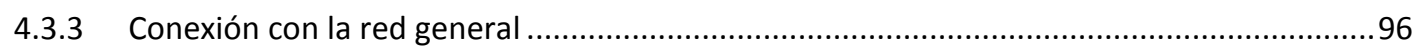

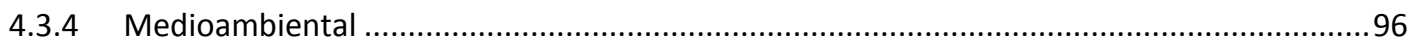

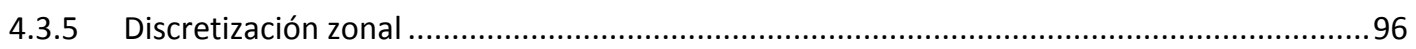

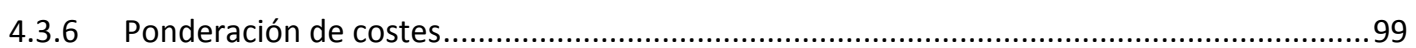

4.4 Pico de demanda máxima de potencia. Situación estacionaria de análisis del

Sistema de energía de tracción (SET). ............................................................ 102

4.4.1 Pasos críticos para analizar un dimensionamiento eléctrico...........................................102

4.4.2 Alternativas para conseguir punto de focalización consistente y fiable ..........................107

4.5 Función objetivo general.............................................................112

4.5.1 Codificación común del modelo de sistema...................................................................114

4.5.2 Codificación/Decodificación Catenaria...............................................................................117

4.5.3 Codificación/Decodificación Subestación de tracción. Grupos de transformación/rectificación de potencia...................................................................................... 118

4.5.4 Codificación/Decodificación Autotransformadores. ........................................................118

4.5.5 Codificación/Decodificación Zonas Neutras. ...................................................................119

4.5.6 Función objetivo de costes de Instalación .....................................................................120

4.5.7 Función objetivo de costes de Operación ..................................................................122

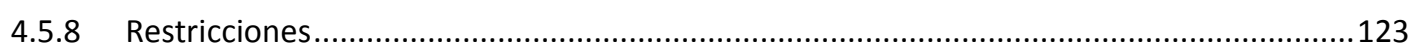

4.6 Conclusiones...................................................................................126 
5. Aplicación de la Metodología en Líneas Ferroviarias de DC. Pruebas y Resultados. 132

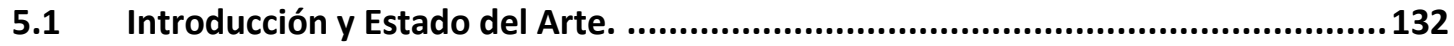

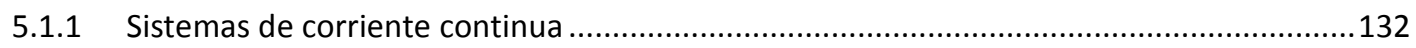

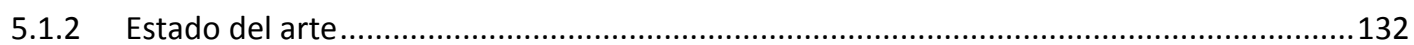

5.2 Líneas ferroviarias en DC para optimizar. Configuración inicial y Resultados.......137

5.3 CASO 1: Línea de Metro. Resultados con algoritmo genético NSGA-II .................139

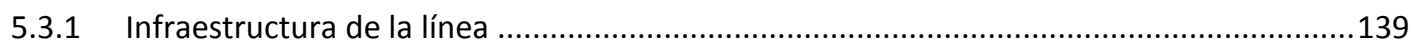

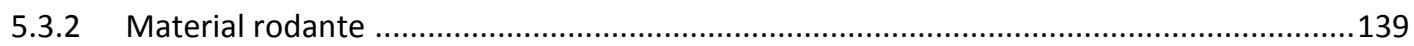

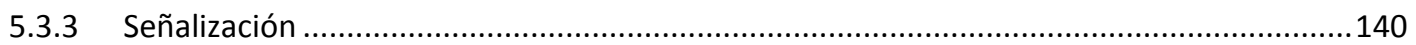

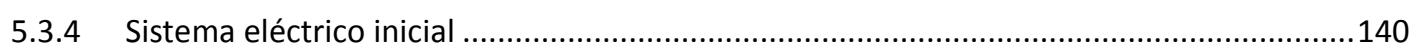

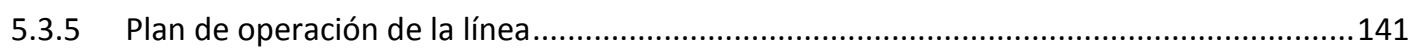

5.3.6 Configuración algoritmo genético. ............................................................................ 141

5.3.7 Resultados eléctricos configuración inicial ..................................................................... 143

5.3.8 Proceso optimización del CASO 1. Configuración y resultados. Análisis. ............................144

5.4 CASO 2: Línea de Metro. Subestaciones fijas. Uso tres técnicas optimización...... 151

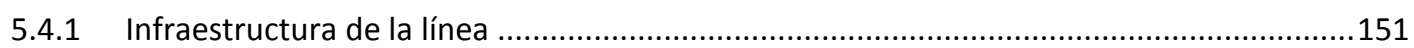

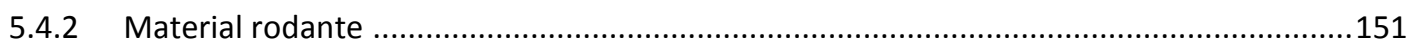

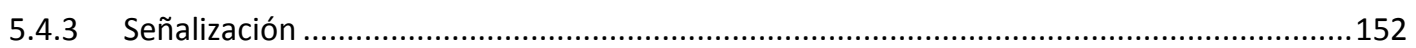

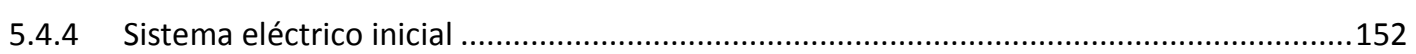

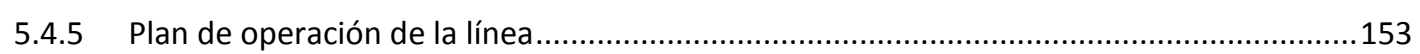

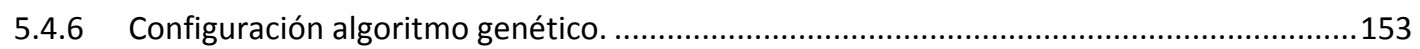

5.4.7 Resultados eléctricos configuración inicial .....................................................................155

5.4.8 Proceso optimización del CASO 2. Configuración y resultados. Análisis. ............................155

5.5 Comparación y conclusiones .................................................................. 182

6. Aplicación de la Metodología en Líneas Ferroviarias de AC. Pruebas y Resultados. 185

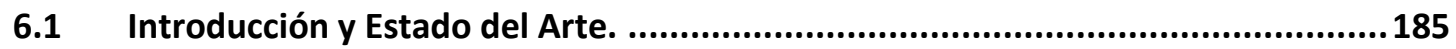

6.1.1 Sistemas de corriente alterna ..................................................................................... 185

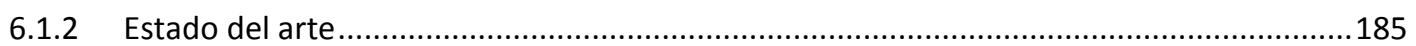

6.2 Líneas ferroviarias en AC para optimizar. Configuración inicial y Resultados. .......190

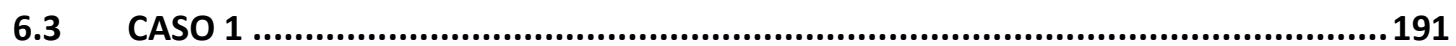

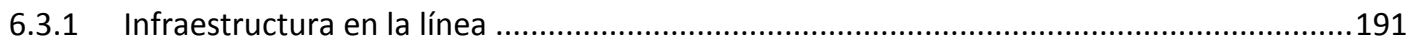

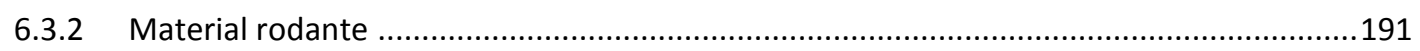

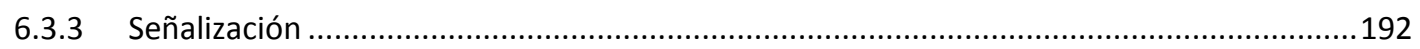

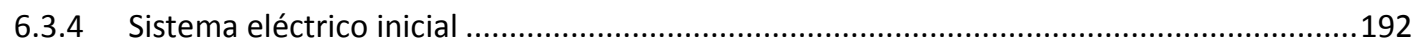

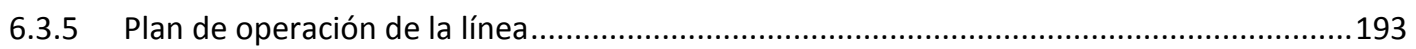

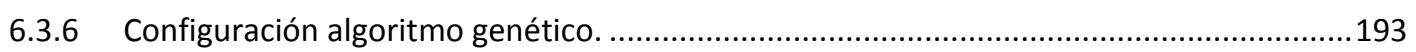

6.3.7 Comentarios configuración inicial .................................................................................. 195

6.3.8 Proceso optimización del CASO 1. Configuración y resultados. Análisis. ............................195

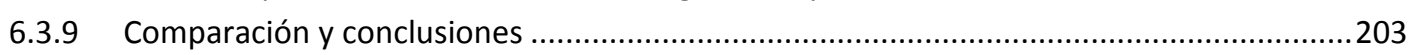

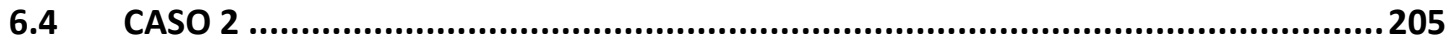

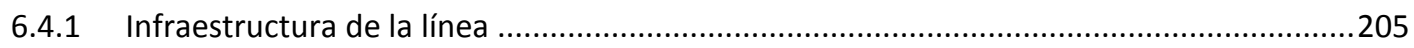

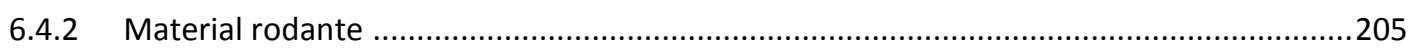




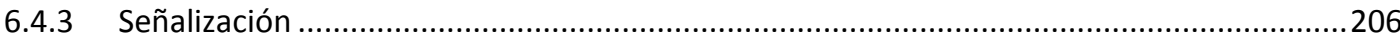

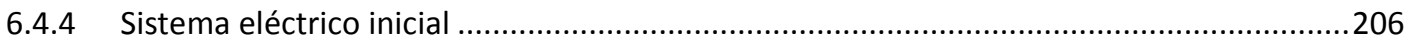

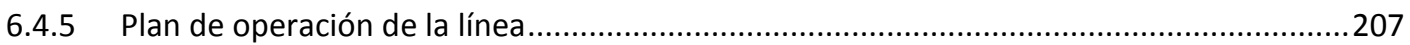

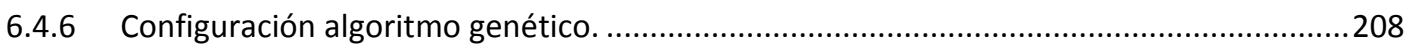

6.4.7 Resultados eléctricos configuración inicial ......................................................................209

6.4.8 Proceso optimización CASO2. Configuración y resultados. Análisis....................................211

6.4.9 Comparación entre ambos ejemplos y conclusiones. ...................................................232

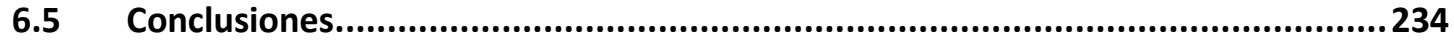

7. Conclusiones. Aportaciones y planteamiento futuro de mejora. Publicaciones $\mathbf{2 3 7}$

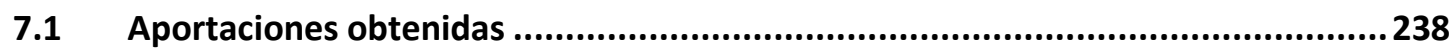

7.1.1 Implantación de Algoritmos genéticos en el proceso de optimización ..............................238

7.1.2 Aplicación directa a sistemas de electrificación .............................................................239

7.1.3 Ponderación y Discretización Zonal ......................................................................................239

7.1.4 Aplicación directa del Pico de Demanda Máxima de Potencia..........................................240

7.1.5 Obtención de resultados aplicando Tests de Convergencia para Restricciones aplicadas en el modelo de optimización .......................................................................................................240

7.2 Planteamiento futuro de la investigación ....................................................241

7.2.1 Incorporar algoritmo genético de última generación........................................................241

7.2.2 Elementos estadísticos en la aplicación de análisis del frente de Pareto..........................241

7.2.3 Proyecciones en las valoraciones de los elementos eléctricos.........................................242

7.2.4 Combinación con sistemas de regeneración de energía ..................................................2242

7.2.5 Combinación con cálculos de optimización de consumo de energía ...................................242

7.3 Publicaciones y visibilidad investigadora ...........................................243 


\section{Introducción. Evolución sistemas de aporte de energía en el ferrocarril. Motivación y objetivos de la Tesis.}

El capítulo tiene una primera parte en la cual se describen los puntos clave de la Tesis. Porque se ha decidido trabajar en esta vía de investigación concreta, qué ha motivado el pensar que era una buena alternativa y la utilidad presente y futura que se pretendía obtener con la aplicación de nuevos conceptos y aplicaciones a la materia escogida.

Por otra parte es interesante lo que plantea el segundo apartado del Capítulo. Se desarrollan de forma resumida el origen, evolución y estado actual de los sistemas de energía aplicados a sistemas ferroviarios, desde el carbón de los primeros días a la electrificación, sistema de energía preponderante en los sistemas actuales.

\subsection{Objetivos de la Tesis y Motivación}

\subsubsection{Motivación}

La movilidad de personas y transporte de mercancías siempre han sido un motivo estratégico para el desarrollo de los países y de su economía. Cuando los materiales fósiles tomaron protagonismo en la generación de movimiento mecánico, en detrimento de la tracción por fuerza animal, se inició una creciente preocupación por la búsqueda de esos materiales fósiles, y en perspectiva hacia futuro, al impacto medioambiental que producía. El ferrocarril es una opción estratégica muy potente que tienen a mano los gobiernos con la que planificar un mejor uso del transporte. La aportación del ferrocarril a nivel social y económico es muy elevada. Algunas de las ventajas inmediatas es por ejemplo, la disminución del tráfico rodado en las ciudades, con lo que conlleva el poder bajar niveles de polución, como a mejorar la movilidad de las personas debido a posibles masificaciones en hora punta. A nivel interurbano también se postula como un medio eficaz para transporte de mercancías y pasajeros. De esta manera el ferrocarril tiene un hueco importante en el tejido social, pero también es importante la inversión a realizar para poner en marcha una línea ferroviaria o para llevar a cabo su explotación diaria. Cada aspecto perteneciente a un proyecto ferroviario tiene un alto coste económico, y para líneas ferroviarias electrificadas, el diseño del sistema correcto es un apartado de gran importancia.

Una de las vías de trabajo e investigación en la que nos hemos centrado a lo largo de los años, ha sido cómo ayudar al diseño del sistema de energía, y más concretamente, a los sistemas ferroviarios que usan la electricidad para generar la tracción.

El sistema eléctrico que finalmente se opte por instalar, será un sistema complejo debido a la interrelación con otros factores tales como la infraestructura ferroviaria, el material rodante o el plan de explotación que se vaya a implantar en la línea. Esta complejidad hace que las posibilidades sean múltiples a la hora de escoger las características principales del dimensionamiento eléctrico que vaya a soportar la toma de energía para tracción. 
La casuística y escenarios posibles que puede plantearse el diseñador suelen ser muy complicados de analizar sin la ayuda de un software aplicado o simulador. Una vez que se tiene y se conoce el simulador, cada opción de escenario posible, debe ser lanzada con la herramienta. A la finalización, se podrán estudiar los resultados. El problema vuelve a ser la ingente cantidad de datos y de casos posibles, combinado con el tiempo que suele tardar cada simulación. Esto puede hacer que el proceso se alargue o peor aun, que se desestimen posibles escenarios por falta de tiempo para su análisis. Por esto y en base a nuestra experiencia en este tipo de estudios, se nos planteó darle un enfoque avanzado y desarrollar una metodología que mejore este proceso. Esta metodología deberá contemplar el máximo de factores posibles que estén involucrados en este tipo de diseños. El resultado final debería aportar a los ingenieros un conjunto de soluciones posibles en base a las características parametrizadas, de modo que tengan un espectro de diseños factibles para sus objetivos y conseguidos en un tiempo razonable. En definitiva, la creación de un sistema experto para la optimización del diseño del sistema eléctrico de tracción.

\subsubsection{Metodología de investigación}

El Centro de investigaciones ferroviarias (Citef) lleva colaborando con empresas dedicadas al ferrocarril desde sus inicios, y desde hace un tiempo, también se trabaja en proyectos de consultoría de dimensionamiento eléctrico ferroviario, tanto para líneas de próxima instalación como para novedades a incluir. Toda la experiencia acumulada ha servido para dotar a la nueva metodología de un empaque práctico y funcional. No obstante se requería de una aproximación mayor al problema.

Hacía falta un trabajo previo de investigación/documentación, para el que se han seguido dos vías. Una vía, es la que se rige por las diferentes normativas existentes relativas al dimensionamiento eléctrico ferroviario. Y la otra vía es la que se encuentra en las distintas publicaciones en revistas y libros científicos. Debido a la complejidad del problema y las distintas áreas que componen el mismo, se ha buscado y recogido mucha información de ambas vías, tanto para conseguir perfilar qué conocimientos serían necesarios para comprender y aplicar en cada área, como para observar el estado del arte en optimización del diseño de sistemas de energía ferroviario.

\subsubsection{Objetivos principales de la tesis}

El objetivo final es conseguir un sistema experto siguiendo una metodología determinada, que sea capaz de aportar valor a la toma de decisiones en cuestiones de diseño del sistema eléctrico de tracción. Para ello, se ha tenido que trabajar en los siguientes temas

\section{Simulador ferroviario multidisciplinar}

La cantidad de datos a manejar, fórmulas matemáticas y la precisión que se quiere conseguir para ser lo más fidedigno posible a las situaciones reales de la explotación 
ferroviaria a analizar, hacen que la manera más realista de atacar el problema sea usando un software especializado, y en este caso de un simulador.

Este simulador deberá ser capaz de trabajar con datos provenientes de:

- Infraestructura ferroviaria. Datos sobre geometría de la línea

- Material rodante. El simulador deberá ser capaz de integrar los parámetros necesarios para poder simular correctamente la dinámica de los trenes. Además no debería estar limitado a una serie concreta de trenes, al contrario, debería ser válido para cualquier tipo de material rodante. La potencia del simulador se corresponde con la calidad y diversidad de adaptación de cada una de sus partes.

- Señalización. La simulación correcta del tráfico existente en la línea y por tanto del plan de explotación establecido. Va a ser necesario contar con un módulo de señalización adaptable al sistema que esté en marcha en la línea a estudiar.

- Sistema eléctrico. Quizá el sistema más complejo debido a la carga matemática que conlleva y también el que más complejidad computacional conlleva. Siguiendo con la filosofía de los demás módulos, el sistema eléctrico también se ha desarrollado para que se pueda aplicar en cualquier sistema ferroviario, y por tanto sistema de electrificación.

En Citef se contaba con un simulador, Hamlet, que cumplía con los requisitos necesarios y expuestos anteriormente.

\section{Búsqueda y adaptación de algoritmos de optimización adecuados para el problema}

Esto forma parte de la investigación y análisis del problema que se está tratando. En las primeras etapas, había que tener muy claro el objetivo final, para posteriormente analizar profundamente los distintos algoritmos y técnicas de optimización que podían ayudar a conseguir esto. También consideramos oportuno que era conveniente tener el apoyo de más de un algoritmo de optimización para poder contrastar los resultados finales, tanto a nivel de evaluación de las funciones objetivo como de respuesta temporal de los mismos. El conocimiento exhaustivo del comportamiento de los algoritmos elegidos es también importante debido a la criticidad temporal del proceso general. Era importante concentrarse en combinaciones adecuadas de los parámetros críticos, para no perder tiempo en resultados finales poco satisfactorios de los algoritmos.

También ha sido necesario la adaptación de los algoritmos al sistema general, es decir al simulador. Como interactuaban y cuál es la forma más adecuada teniendo en cuenta las propiedades del simulador, es otro reto al que nos hemos enfrentado. 


\section{Acotar las variables críticas del modelo de optimización. Discretización zonal y ponderación de costes}

Como se ha explicado anteriormente, un proyecto de implantación inicial total o de modificación parcial de un sistema ferroviario implica muchos factores. Por una parte se tenía que buscar qué podía interesar más al diseñador/ingeniero de cara a obtener datos mesurables en la solución final. Por otra parte cómo se podría dar valor a esos conceptos que en una primera aproximación es muy complicado de obtener el precio exacto y quizás no sea lo más importante. Por este motivo, una vez especificados los aspectos que el sistema será capaz de aceptar y procesar, se pensó en cómo adaptarlo por medio del concepto de Discretización zonal. En cuanto a los elementos particulares también se exigía un tipo de valoración de cada uno de ellos, se optó finalmente por asociarles una ponderación en base a los valores reales de cada uno.

\section{Funciones objetivo y restricciones críticas inherentes al modelo}

Si se sigue secuencialmente el orden de los objetivos detallados hasta este punto, se puede comprobar que es necesario concretar un modo de computar el valor de cada escenario o diseño de dimensionamiento eléctrico propuesto. Ha hecho falta concretar en dos funciones objetivo, contrapuestas ambas entre sí, las características ponderadas anteriormente comentadas y la cantidad de cada una de ellas. También se ha integrado en una de las funciones objetivo un índice de calidad del sistema eléctrico.

Los sistemas de dimensionamiento eléctrico, según normativa, tienen un alto grado de restricciones que han de cumplir, tanto por tema de viabilidad del sistema como por temas de seguridad. En los algoritmos genéticos integrados en el trabajo, contienen la posibilidad de incluir restricciones que penalizan en caso de no cumplirlas. Esto ha sido tenido en cuenta, y se han adaptado algunas restricciones del sistema eléctrico para de esta manera, mejorar la toma de decisiones. También se ha pensado en incluir en las restricciones, una que limite el montante total de coste del dimensionamiento elegido. Todo esto para hacer más fiable el conjunto de soluciones finales.

\section{Análisis y búsqueda de criterios que sirvan para determinar y conocer el estado del sistema eléctrico para la tracción ferroviaria}

Lo que se trata en este punto es de cómo conseguir un criterio que sirva para valorar el impacto del plan de explotación sobre el dimensionamiento eléctrico de la línea. Es decir, el análisis de los posibles escenarios ferroviarios con los que se pueda trabajar con esta metodología, deben tener un denominador común en cuanto a conocer el sistema en general, estudiando solo una parte. Por eso se ha llamado criterio de expansión del comportamiento.

Este criterio finalmente, es el que calcula los picos de demanda máxima de potencia, que en el apartado correspondiente se detallará las características concretas, así como el proceso que se ha seguido para llegar a escogerlo. 
Integración general de las distintas áreas. Comprobación de la validez del sistema.

Por último, tener todos los actores del sistema funcionando correctamente por separado y una vez comprobado que funcionaban correctamente y que tenían sentido, lo próximo era integrarlo todo en el sistema común.

Las pruebas a realizar, teniendo en cuenta la multiplicidad de casos que se pueden dar, se ha tratado de conseguir datos reales de sistemas reales o en estudio. También se han probado los tres sistemas de electrificación que se han contemplado: corriente continua (DC), corriente alterna (AC) tanto simple (1x25) como dual (2x25).

Para la validez del sistema, obviamente, con los resultados finales y con el simulador ferroviario, se han escogido escenario propuestos y analizados para ver si cumplían la normativa y regulación ferroviaria. También era necesario obtener tiempos de respuesta de los algoritmos y testear el comportamiento de cada uno de ellos dependiendo de las circunstancias o parámetros iniciales. Todo esto debía ser satisfactorio, tanto en validez de los escenarios propuestos, como en el tiempo para la obtención de los mismos. 


\subsection{Sistemas de energía aplicados a líneas ferroviarias. Breve repaso histórico y evolución.}

Desde la aparición de la máquina de vapor, y desde que se aplicó a un sistema de transporte que se trasladaba por raíles, se ha ido pensando en cómo mejorar las prestaciones y también cómo adaptar las nuevas tecnologías al ferrocarril. Entre las evoluciones aplicadas están obviamente, las referidas al sistema de energía directa en los trenes. Es interesante detallar los principales momentos y qué tecnología o material hizo que los cambios de etapas fueran posibles, y otorgándole al ferrocarril el estatus que tiene hoy en día.

\subsubsection{Introducción}

A lo largo de la historia uno de los retos de las distintas civilizaciones ha sido encontrar una forma de mejorar los medios de transporte para movilizar tanto mercancías como personas. La aparición del ferrocarril a principios del siglo XIX [1], fue un claro punto de inflexión en la manera de ver el transporte, el comercio y viajes de personas. Esto hizo que las distancias fueran menores y supuso un surgimiento de una fuerte y pujante competencia con los otros medios de transportes de la época, y que provocara el auge de la investigación en tecnología asociada al nuevo medio de transporte, y por supuesto, a las nuevas formas de dotar de energía al mismo.

Hasta la fecha, el transporte ferroviario ha visto como el sistema de energía principal del que se nutre la tracción, ha ido adaptándose a la tecnología del momento. Vapor, Diesel, Eléctrico componen esa evolución compartida con las necesidades de las aplicaciones derivadas del ferrocarril. Actualmente existen algunas líneas ferroviarias usando levitación magnética [2], aunque de momento no están extendidas.

\subsubsection{Sistemas de energía aplicados}

\subsubsection{Vapor}

Los comienzos del transporte masivo de personas y mercancías por ferrocarril, tal y como lo conocemos hoy en día, comenzó teniendo la máquina de vapor como generador de energía. Los primeros intentos de evolución de la idea de una máquina que moviera aparatos mecánicos sin la actuación directa humana o animal, fue derivada de la necesidad del sector del metal, en las minas. A pesar de que ya se habían hecho algunos experimentos teniendo en cuenta las posibilidades para generar movimiento por parte del vapor [3], se toma como hito inicial y como persona más influyente debido a sus patentes a J. Watt [4]. Trabajando sobre un nuevo modelo de condensador, el vapor era condensado y separado por un cilindro. Esto mejoró las prestaciones de los trabajos anteriores. Las patentes conseguidas por Watt fueron claves en mejorar los procesos productivos, en general, no solo del transporte, y por supuesto fue clave en la Revolución Industrial del siglo XIX. 
Los cambios producidos se expandirían en todas las direcciones del nuevo tejido productivo. Entre estos cambios, y de forma particular, estarían las mejoras que se instauraron en el transporte de las materias primas primero, y posteriormente de personas en las minas. Uno de los pioneros, el científico inglés R. Trevitchik [5] propuso un nuevo artefacto de generación de energía móvil, las locomotoras aplicadas al transporte en las minas de hulla. Esto hizo mucho más eficiente el trabajo realizado. El nuevo medio de locomoción tenía carencias que imposibilitaban su aceptación a nivel más global, fuera de las minas.

El transporte por raíles no es un invento que se iniciara con el ferrocarril. Existen muchos ejemplos desde la Antigüedad que así lo atestiguan. El ferrocarril lo que introduce como novedad, es la ausencia de fuerza de arrastre animal y el cambio a materiales más resistentes para actuar como raíles. Tal y como se ha explicado, el ferrocarril tuvo gran importancia en los progresos de la Revolución Industrial. El transporte masivo de mercancías por terrenos generalmente, embarrados y con índices altos de rugosidad, hacían muy costosos los viajes. En este punto es donde los avances producidos a lo largo de los años usando la máquina de vapor, los prototipos e ingenios mecánicos, serían tenidos en cuenta para fomentar un nuevo medio de locomoción.

En la Inglaterra de principios de siglo XIX, G. Stephenson [6], mejoró el sistema de transporte por raíles usando la máquina de vapor, con el objetivo de dar más valor a los trabajos en las minas de carbón. Después de los intentos fallidos anteriores, la nueva locomotora ideada por Stephenson, inició su andadura en el año 1814. Estos primeras máquinas de arrastre que utilizaban las ideas de Watt, fueron aplicadas, como anteriormente, al transporte de materiales en las minas. No fue hasta el año 1825 que se aplicó al movimiento de personas. El trayecto entre las ciudades inglesas de Stockton y Darlington fue el primero. Cinco años más tarde, en 1830, se inauguró la primera línea ferroviaria con tráfico mixto de pasajeros y mercancías entre las ciudades de Manchester y Liverpool. La locomotora Rocket fue la pionera, desarrollada por Stephenson, que se muestra en la Figura 1.

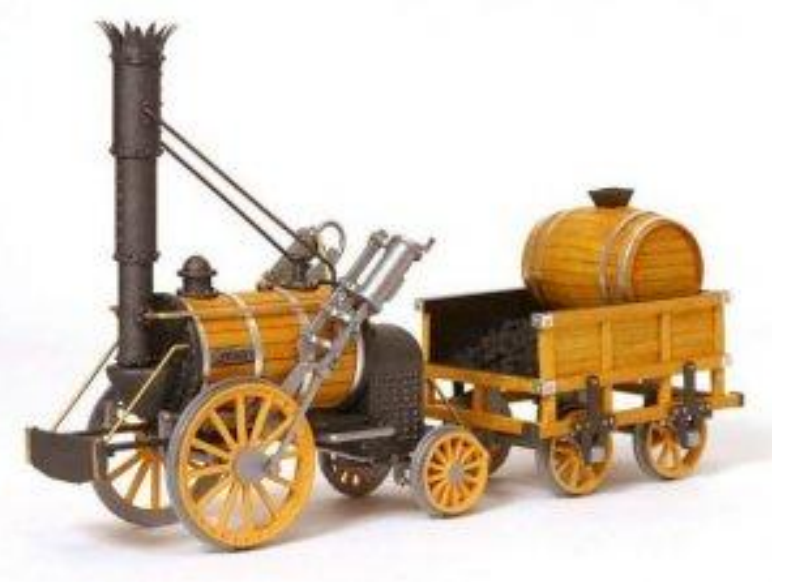

Figura 1 Modelo ilustrativo de la primera locomotora para tráfico ferroviario con pasajeros. Locomotora Rocket. 
La locomotora de vapor es una máquina robusta y sencilla. El vapor pasa; a presión, de la caldera a los cilindros, donde se expande y empuja al pistón hacia el otro extremo. En la carrera de retorno, se abre una lumbrera para dar salida al vapor. El recorrido alternativo del pistón se transforma en un movimiento giratorio gracias a las bielas, que mueven las ruedas de la locomotora.

El éxito obtenido en las primeras líneas ferroviarias, hizo que se expandiera a demás países de Europa y Norteamérica. En España la primera línea ferroviaria se inauguró en Cuba, por entonces pertenecía a la Corona Española. Se construyó entre 1835 y 1837, y cubría el trayecto entre La Habana y Bejucal. En la Península Ibérica el primer ferrocarril no llegó hasta 1848 y circulaba entre Mataró y Barcelona. Pero esta expansión del nuevo medio de locomoción, a pesar de las evidentes mejoras respecto de los anteriores medios de transporte, tenía dificultades (pesos muy altos, baja velocidad, dificultad de mantenimiento de carriles, etc...) que con el paso del tiempo y aparición de nuevas formas de energía aplicadas, harían que el transporte por máquina de vapor, fuera dando paso a otras.

\subsubsection{Diesel}

R. Diesel [7] a finales del siglo XIX, con la patente registrada, elaboró un sistema motorizado de combustión interna que proporcionaba energía para movimiento mecánico. Al igual que para el sistema de máquina de vapor, la adaptación al ferrocarril no fue inmediata.

Las primeras locomotoras que aplicaron este tipo de transmisión de la energía a la fuerza motriz, se sitúan en Suecia a principios del siglo XX [8]. El desarrollo de prototipos se frenó debido a la I Guerra Mundial, y no se volvió a retomar los trabajos de investigación hasta mediados los años 20 del siglo pasado. Después de este lapso de tiempo de inactividad en la progresión del trabajo con este tipo te tecnología, el Profesor ruso Yuri Lomonossoff (Figura 2), dotó al sistema inicial de mucha más eficiencia, ya que mejoró los problemas de transmisión aplicándole electricidad. Esta mejora hizo posible la implantación generalizada en el sistema ferroviario, y de esta manera, comenzar a competir con las locomotoras accionadas por máquinas de vapor 


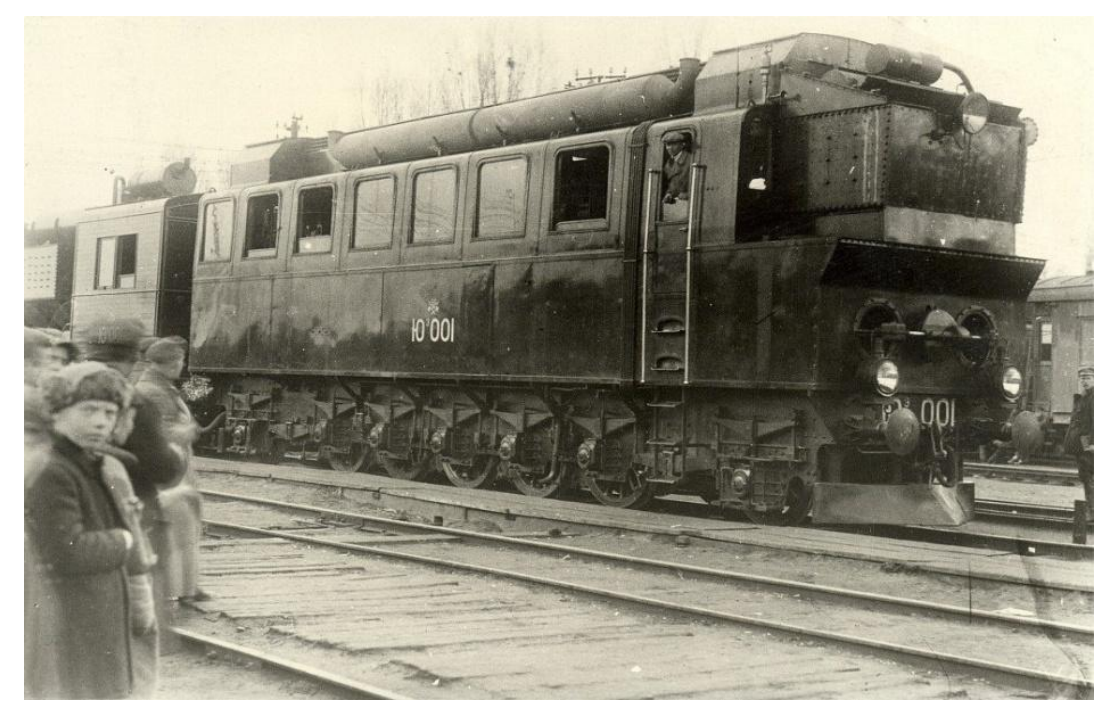

Figura 2 Locomotora ideada por Y. Lomonossoff propulsada por diesel.

Las locomotoras diesel son principalmente de estos tres tipos: diesel-eléctricas, diesel-hidráulicas y diesel-mecánicas. En la locomotora diesel-eléctrica, el motor diesel mueve una dinamo que alimenta de energía eléctrica a los motores eléctricos que mueven las ruedas. En diesel-hidráulica, el motor acciona una transmisión hidráulica que hace girar las ruedas mediante una especie de turbina en miniatura. Por último, en la diesel-mecánica, la transmisión se efectúa a través de una caja de engranajes, igual que en un automóvil. Las diesel-eléctricas son, con mucho, las más empleadas, aunque en algunos casos, se utilizan también las diesel-mecánicas para maniobras.

Las primeras locomotoras accionadas por diesel, fueron las combinadas con la electricidad. Con un motor de 6 cilindros podían alcanzar una potencia de $75 \mathrm{CV}$, a 55 rpm y acopladas a un generador de corriente continua (DC). Las prestaciones lógicamente fueron aumentando durante los años posteriores a la Gran Guerra, y los distintos países iban aceptando la nueva modalidad. En la década de los 30, en Alemania se puso en servicio el primer tren de "alta velocidad diesel-eléctrico" denominado "Flying Hamburguer". La implantación del convertidor de flujo eléctrico permitió un uso más económico del diesel. El salto en las prestaciones fue considerable, $820 \mathrm{CV}$ y $1400 \mathrm{rpm}$. Posteriormente en 1936, un material rodante de 3 coches articulado, era capaz de alcanzar los $127 \mathrm{~km} / \mathrm{h}$. La expansión de los trenes dieseleléctrico se hizo efectiva en los demás países europeos. En Estados Unidos, la empresa General Motors, en los años 30, ayudó enormemente al auge de esta tecnología.

Se sitúa en la década de los años treinta la entrada de las locomotoras con tracción diesel en España. La competencia con el tráfico rodado por carretera, hizo que las compañías ferroviarias entraran en crisis y tuvieron que adaptarse a las nuevas condiciones, intentando ser más competitivos. Las primeras máquinas diesel que se pusieron en marcha, fueron sobre todo para líneas secundarias y con poco tráfico. La Guerra civil y la postguerra pospusieron el auge del diesel. No fue hasta la década de los cincuenta-sesenta cuando comienzan a circular con más frecuencia trenes diesel para maniobras y para trenes de pasajeros, sobre todo diurnos con tráfico débil. En la 
actualidad, la mayor parte de tráfico ferroviario con diesel es para transporte de mercancías.

Al igual que pasó con el paso de la predominancia de la máquina de vapor hacia la otra forma de energía, el diesel, la electricidad como forma única de generación de energía de transmisión también se fue imponiendo. En la actualidad la locomoción Diesel aplicada a ferrocarriles para transporte de viajeros está en retroceso en favor de la electricidad como único sistema de energía. Es sobre todo en el transporte de mercancías donde las locomotoras Diesel tienen más vigencia en la actualidad.

\subsubsection{Eléctrica}

La tercera de las formas principales de locomoción es la tracción eléctrica. En el siglo XIX, al igual que para la máquina de vapor, también fue el inicio de la evolución masiva de la aplicación de la energía eléctrica a la vida cotidiana. En términos ferroviarios, hubieron algunos intentos pero fracasaron, hasta el descubrimiento por parte de W. von Siemens y Ch. Wheatstone en 1866 del principio de la Electrodinámica. Esto permitía generar grandes cantidades de energía efectiva, y por tanto multiplicar sus posibles aplicaciones. En 1879, Siemens mostró en la Feria de Comercio de Berlín (Figura 3), la primera locomotora eléctrica, con un motor DC de $2.2 \mathrm{~kW}$. Aunque no fue finalmente hasta 1895 que se instauró la primera línea completamente eléctrica entre Baltimore-Ohio.

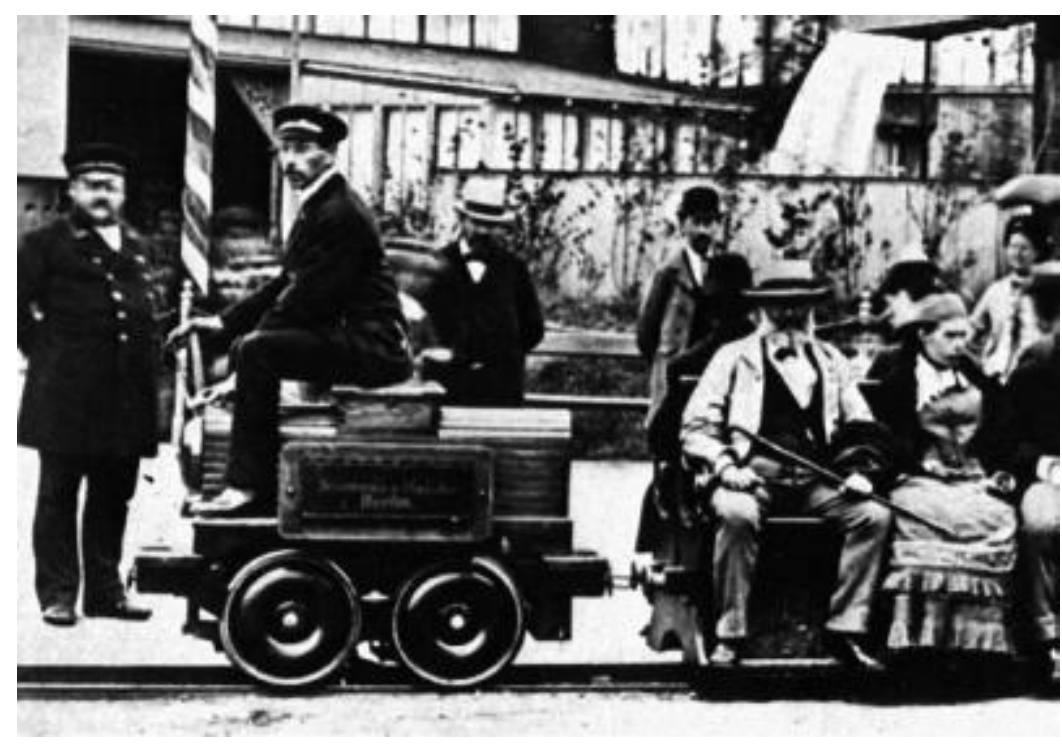

Figura 3 Feria de Comercio de Berlín 1879. Primera locomotora eléctrica desarrollada por W. Siemens

La motivación inicial para llevar adelante el proyecto de las líneas ferroviarias eléctricas, se basó principalmente, en evitar la emisión del monóxido de carbono por parte de las máquinas de vapor y también para mejorar la potencia que necesitan los grandes gradientes que tenían que subir los trenes, en definitiva, para sistemas ferroviarios en montaña. El Metro de Londres es el primer metro electrificado [9], y trataba de mejorar de esta manera los problemas derivados de la emisión de gases, la cual exigía un complicado sistema para tener que evacuarlos, por medio de sumideros 
espaciados a lo largo de la línea, por los que se dejaban salir los gases almacenados. Para la construcción en 1905 del túnel entre Brig (Suiza) e Iselle (Italia), tramo de 20 $\mathrm{km}$, que pasaba por los Alpes, concluyeron que la inhalación de monóxido de carbono por los pasajeros hacía inviable otro tipo de tracción que no fuera la eléctrica. La línea ferroviaria que subía al Lötschberg, tenía que atacar gradientes de porcentajes entre el 2.2 y el 2.7, además de radios de 300m. Se optó por tracción eléctrica y se terminó en el año 1913 , y con sistema de $15 \mathrm{kv}$ y $15 \mathrm{~Hz}$.

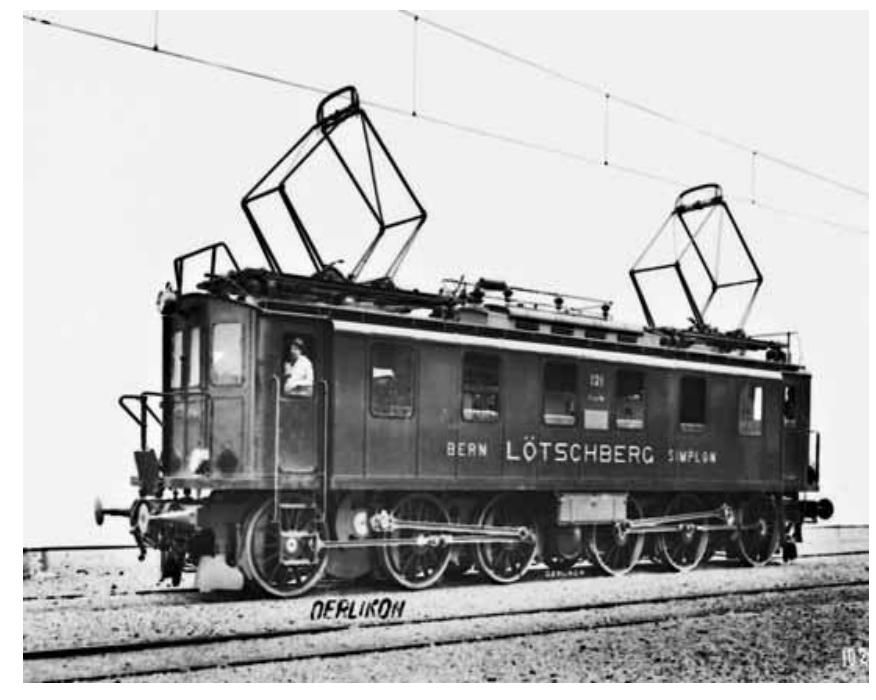

Figura 4 Serie Lötschberg año 1913

Las primeras locomotoras eléctricas funcionaban con baterías [10], pero las modernas toman la corriente de un cable aéreo o de un tercer raíl. En este último sistema, la corriente es suministrada por el raíl suplementario y retorna por los de rodadura. También se emplean sistemas de cuatro carriles, dos de ellos para alimentación y retorno de la corriente.

Para determinar el sistema de electrificación que se está usando en una línea determinada, se suele referir al sistema de corriente aplicado, corriente continua o alterna. A continuación se explicará brevemente la aparición, evolución y aplicación de cada una de ellas.

\subsubsection{Sistemas de tracción con corriente continua}

Las tensiones de trabajo para corriente continua dependen de cada sistema ferroviario en cada país. El conjunto de sistemas de alimentación trabajan con alguno de los siguientes: $\{600,750,900,1200,1500,3000\}$ [11]. Las distancias entre subestaciones, debido a las caídas de tensión y altas corrientes, suele ser pequeñas. Para líneas de corta distancia o de cercanías el rango suele estar entre los 5 y los $10 \mathrm{~km}$. Para sistemas metropolitanos no suelen ser mayores de $2-5 \mathrm{~km}$. Para líneas con más alto voltaje, las subestaciones pueden estar incluso separadas por $20 \mathrm{~km}$. Dependiendo por supuesto de la potencia máxima demandada por los trenes, el tráfico de la línea y la geometría de la misma. También suelen estar implantado en sistemas tranviarios y PRT/MRT. 
Las bajas tensiones de trabajo, que hacen más sencillo la adaptación a los motores embarcados de los trenes, hace muy compleja la transición entre las altas tensiones de la red de distribución y los valores que van a circular por el tercer carril. Esta complejidad en la transformación incrementa los costes de la explotación.

El sistema eléctrico de las subestaciones de tracción suele estar conformado por diversos transformadores de potencia, que se adaptan a la capacidad necesaria para la línea. Una vez que la tensión alterna proveniente de la red general se ha adaptado a la diseñada para la línea, o sea se tiene ya en baja tensión, las subestaciones de sistemas en DC, suelen tener puentes rectificadores de potencia.

En España, el sistema de $3 \mathrm{kV}$ es el elegido mayoritariamente para las redes de corriente continua de ferrocarril y de $750 \mathrm{~V} / 1500 \mathrm{~V}$ para metros. Mientras que en el resto de Europa, desde inicios del siglo XIX, fueron conformando su propio sistema de electrificación y tensión relativa.

\subsubsection{Sistemas de tracción con corriente alterna}

La implantación de la corriente alterna en sistemas ferroviarios trata de paliar los inconvenientes de los sistemas a baja tensión. Principalmente, con corriente alterna, alta tensión, las corrientes son menores y por tanto los conductores se calientan bastante menos siendo menores las pérdidas provocadas, por lo que es posible mantener una intensidad de corriente durante una longitud mayor. Por lo que para explotaciones de media y larga distancia es muy recomendable usar AC. Las grandes distancias deben ser asequibles a la confortabilidad y necesidades de los pasajeros, por lo que tienden a implantarse sistemas de alta velocidad en ellas. Los trenes que circulan demandan potencias elevadas, por lo que este sistema es muy adecuado, permitiendo esas prestaciones. Además permite no depender tanto de subestaciones cercanas como en DC. Las subestaciones se pueden espaciar mucho más.

Al estar trabajando en sistema trifásico el planteamiento para difuminar lo más posible el efecto de posibles desequilibrios en la línea, es dividir la misma en sectores eléctricos, de modo que cada uno pertenezca a una subestación de tracción con distinta fase.

Las conexiones con la red general se suelen efectuar a redes de $132 \mathrm{kV}, 220 \mathrm{kV}$ ó $400 \mathrm{kV}$. Las tensiones de alimentación aplicadas son:

- $\quad 15 \mathrm{kV} / 162 / 3 \mathrm{~Hz}$

- $\quad 25 \mathrm{kV} / 50 \mathrm{~Hz}$

- $50 \mathrm{kV}$

En España el sistema de electrificación elegido es el de $25 \mathrm{kV} / 50 \mathrm{~Hz}$. Está implantado en todas las líneas de alta velocidad que se han construido en los últimos 20 años. La instalación suele ser con el sistema bitensión, también llamado 2x25. Gracias a la implantación de autotransformadores a lo largo de la línea y de conductores llamados feeder de retorno, las intensidades que recorren la catenaria son mucho menores, y la 
tensión nominal es de $50 \mathrm{kV}$. Como mayor aportación de este sistema, y que está tan extendido en países como Francia, Japón, Italia, Rusia, es que permite una distanciación mayor entre subestaciones de tracción. Otra ventaja sobre el otro sistema es que las perturbaciones electromagnéticas son mucho menores. En cambio es un sistema mucho más caro de instalar y de mantener.

\subsubsection{Comparativa general entre sistemas de generación de energía ferroviaria.}

Llegados a este punto, se han resumido en la siguiente tabla los motivos por los que se ha ido consolidando el sistema de tracción eléctrico en detrimento de los otros dos, especialmente el que trabaja con máquina de vapor, que prácticamente está en desuso.

- Tracción eléctrica, ventajas e inconvenientes respecto los otros sistemas

- Frente a la máquina de vapor [12]

\begin{tabular}{|c|c|}
\hline Ventajas & Inconvenientes \\
\hline Mayor par de arranque & $\begin{array}{l}\text { Fuertes gastos de primer } \\
\text { establecimiento }\end{array}$ \\
\hline $\begin{array}{l}\text { Reversibilidad de motores - } \\
\text { regeneración de energía }\end{array}$ & $\begin{array}{c}\text { Mayores cargas financieras en } \\
\text { la explotación ferroviaria }\end{array}$ \\
\hline Mayor adherencia & $\begin{array}{c}\text { Influencia grande ante averías } \\
\text { de la transmisión de energía }\end{array}$ \\
\hline Gran aceleración & \\
\hline Mayor potencia específica & \\
\hline $\begin{array}{l}\text { Acoplamiento de muchos } \\
\text { motores }\end{array}$ & \\
\hline $\begin{array}{l}\text { Ausencia de monóxido de } \\
\text { carbono }\end{array}$ & \\
\hline Menor ruido ambiente & \\
\hline Mayor capacidad de tráfico & \\
\hline $\begin{array}{l}\text { Líneas de montaña, túneles, } \\
\text { fuertes rampas, etc.. }\end{array}$ & \\
\hline $\begin{array}{l}\text { Estaciones terminales y } \\
\text { subterráneas }\end{array}$ & \\
\hline $\begin{array}{l}\text { Escasez de combustibles } \\
\text { fósiles }\end{array}$ & \\
\hline
\end{tabular}

Tabla 1 Ventajas/inconvenientes vapor-eléctrico 
- Frente a la tracción diesel-eléctrica [13]

\begin{tabular}{|c|c|}
\hline $\begin{array}{c}\text { Pueden generar más CV } \\
\text { que el Diesel }\end{array}$ & $\begin{array}{c}\text { Coste inconicial de locomotoras } \\
\text { sensiblemente mayor al de las } \\
\text { Diesel }\end{array}$ \\
\hline $\begin{array}{c}\text { No tiene motor primario } \\
\text { (Diesel) }\end{array}$ & $\begin{array}{c}\text { Independencia de la línea de } \\
\text { electrificación. Puede circular } \\
\text { en cualquier infraestructura }\end{array}$ \\
\hline $\begin{array}{c}\text { Costes de mantenimiento } \\
\text { más bajo }\end{array}$ & $\begin{array}{c}\text { Coste inicial mayor por } \\
\text { instalación eléctrica } \\
\text { suplementaria (catenaria/tercer } \\
\text { carril, subestaciones, etc..) }\end{array}$ \\
\hline Mejor disponibilidad & \\
\hline Mayor durabilidad & \\
\cline { 1 - 1 } $\begin{array}{c}\text { No dependencia de material } \\
\text { fósil }\end{array}$ &
\end{tabular}

Tabla 2 Ventajas/Inconvenientes diesel-eléctrico

Los datos en la actualidad de vía electrificada respecto del total varían según los países de nuestro entorno. Mientras que en Suiza es del 100\%, en Gran Bretaña solo es del $40 \%$. En término medio estarían casos como el de España, Italia, Suecia y Holanda, cercanos al 70\%, y otro ejemplo con un poco menos de desarrollo de electrificación en la red ferroviaria sería Alemania, con un 59\%.

\subsubsection{Implantación general del sistema de tracción eléctrico ferroviario e impacto social y económico. Situación actual.}

El impacto que tuvo el ferrocarril en la sociedad es evidente que fue muy elevado. Un síntoma esclarecedor es la rápida expansión que tuvo en los países que entraban en contacto con las líneas ferroviarias del momento. En España, donde en general los caminos existentes no tenían condiciones para poder desarrollar velocidades elevadas, la implantación de los sistemas ferroviarios trajo grandes cambios. Por supuesto el movimiento de materias primas implicó gran desarrollo industrial. Pero no hay que obviar el desarrollo social que produjo. Las comunicaciones interpersonales mejoraron, el correo llegaba con mucha más celeridad, las personas se podían trasladar de forma más eficiente a las ciudades, con lo que se inició un movimiento migratorio que aumentó el tamaño de las mismas. Solo en España en los primeros 50 años de implantación, existen estudios que cifran en ahorros de hasta los 2500 millones de pesetas de la época.

En la actualidad existen otras connotaciones que siguen haciendo del ferrocarril un sistema de locomoción muy interesante tanto a nivel económico como social. A nivel social, la diversidad de medios de transporte que existen no menoscaba la necesidad del ferrocarril. La opción de evitar el movimiento de personas por carretera, además de disminuir el número de accidentes, disminuye también la polución, notándose sobre todo en grandes ciudades. 
Este impacto del que se habla, debe ser tratado estadísticamente para que pueda llegar a tener una comprensión mejor del alcance que tiene. Comparativamente se pueden y de hecho, se realizan estudios que permiten conocer la idoneidad de implantar un medio de transporte en contraposición a otros. Ejemplos y estudios como los que se presentan en las tablas siguientes.

Los datos presentados a continuación pertenecen a un proyecto ferroviario entre las ciudades de Alejandría y El Cairo [14].

\begin{tabular}{ccc}
\hline Tipo instalación & Cantidad & $\begin{array}{c}\text { Coste estimado total } \\
\text { (millones euros) }\end{array}$ \\
\hline Subestaciones & 5 & 100 \\
Catenaria & $236 \mathrm{~km}$ & 216 \\
Talleres & & 200 \\
Señalización & & 50 \\
Trabajo obra civil & & 250 \\
(infraestructura) & & 816 \\
\hline Total & & \\
\hline
\end{tabular}

Tabla 3 Ejemplo coste proyecto sistema eléctrico ferroviario

En la Tabla 3, se aprecia el coste significativo que tiene el sistema eléctrico dentro de un presupuesto de implantación de una nueva línea ferroviaria. Esto conlleva que un buen diseño del mismo, puede constituir un ahorro apreciable en el presupuesto y hacer mucho más competitivo el nuevo medio de transporte entre origen-destino.

\begin{tabular}{ccccc}
\hline \multirow{2}{*}{$\begin{array}{c}\text { Tipo de } \\
\text { emisión }\end{array}$} & \multicolumn{2}{c}{ Transporte por carretera } & \multicolumn{2}{c}{ Ferrocarril } \\
\cline { 2 - 5 } & $\begin{array}{c}\text { Coche } \\
\text { privado/taxi }\end{array}$ & Autobús & Diesel & Eléctrico \\
$\begin{array}{c}\text { Dióxido de } \\
\text { carbono }\end{array}$ & 126.7 & 35 & 92.1 & 68.4 \\
$\begin{array}{c}\text { Oxido nitroso } \\
\text { Monóxido de }\end{array}$ & 1.16 & 0.39 & 0.88 & 0.32 \\
$\begin{array}{c}\text { carbono } \\
\text { Hidrocarburos }\end{array}$ & 0.57 & 0.29 & 0.62 & 0.030 \\
\hline
\end{tabular}

Tabla 4 Comparativa medios de transporte afectación gases emitidos

Los resultados recogidos y resumidos en la Tabla 4, muestran claramente la razón del interés existente en fomentar el transporte por ferrocarril, focalizado en este caso, en el bajo impacto medioambiental que genera respecto de los otros grandes medios de transporte.

Conforme ha ido aumentando la instalación de nuevas líneas ferroviarias de alta velocidad, se han desarrollado estudios e investigaciones que apoyan con datos objetivos, el auge de este medio de transporte. En Tabla 5 se pueden observar los valores tan significativos, por ser bastante mejores, respecto al tráfico rodado y al avión, que ofrece el uso de la alta velocidad ferroviaria [15] [16]. En la Tabla se muestran los costes marginales externos (accidentes, ruido, polución, cambio climático, impacto en 
las ciudades) en situación de no congestión de tráfico. Se puede ver que la alta velocidad es para trayectos cortos, hasta casi una cuarta parte de los costes de coche y avión.

\begin{tabular}{ccc}
\hline Transporte & Trayecto Paris-Viena & Trayecto París-Bruselas \\
\hline Coche & 40.2 & 43.6 \\
Tren & 11.7 & 10.4 \\
Avión & 28.7 & 47.5 \\
\hline
\end{tabular}

Tabla 5 Costes marginales externos en ( $\frac{\text { euros }}{1000}$ pasajero $\left.-\mathbf{k m}\right)$ uso de transporte público en tramos entre dos grandes ciudades

En relación con el trabajo último referenciado, también aporta un dato acerca del coste habitual de superestructuras en proyectos ferroviarios. En [17], establecen que alrededor del $10 \%$ del coste total, se suele dotar para la electrificación.

En un mundo donde las políticas energéticas cada vez son más importantes e influyentes en las tomas de decisión de los gobiernos, esas decisiones se tornan más relevantes. Y conjuntamente, la energía eléctrica con el ferrocarril, hace que la importancia sea muy elevada y que cualquier elección debe ser analizada, mesurada y con poco margen de error. El trabajo que se presenta en esta Tesis es sensible a esta necesidad y trata de mejorar el proceso de diseño en tiempos de respuesta, costes y pérdidas de energía.

\subsubsection{Características básicas Sistema eléctrico de tracción ferroviaria}

El diseño del sistema eléctrico para la tracción tiene unas pautas y características básicas que se deben tener en cuenta. El objetivo de este apartado es enumerar los elementos básicos que se tienen en cuenta en el diseño, detallar apuntes acerca de la normativa o regulación que se suele seguir para diseñar y por último comentar la distinción entre los dos sistemas de electrificación existentes.

Tal y como se ha explicado anteriormente, la potencia de computación que existe actualmente ha hecho que los análisis de los posibles casos o escenarios de dimensionamiento eléctrico, tengan más capacidad. Para estudios con estas características, los diseñadores hacen uso de simuladores. La gran cantidad de datos, elementos clave y situaciones relacionadas, son las que se van a enumerar a continuación, para así dar una visión clara de la dimensión del problema que se está tratando y que se ha buscado mejorar el trabajo gracias a las investigaciones de esta Tesis.

\section{Geometría de la línea}

En la transición de los trenes de vapor a trenes Diesel, uno de los factores determinantes en los que se incidió para modificar el dominio de los anteriores, es la posibilidad mayor para subir grandes pendientes que ofrecía la tracción Diesel-eléctrica. La geometría de la línea es determinante tanto a la hora de calcular la demanda del 
tráfico, como, en el caso que nos ocupa, del dimensionamiento eléctrico, de los equipos que van a tener que ser instalados a lo largo de la vía para dar soporte energético.

No solo las rampas van a tener una incidencia en la demanda de los trenes. Todas aquellas fuerzas resistivas asociadas a una línea ferroviaria, inciden en este aspecto (p.ej. la fuerza a paso por túnel o resistencia al avance).

\section{Tipo de señalización/explotación ferroviaria}

Para la correcta realización de un diseño, se ha de contar con el tipo de señalización que se va a implantar a lo largo de la línea ferroviaria. Es evidente que el consumo de energía por parte del sistema de señalización no ocupa un porcentaje significativo, aunque lógicamente se debe tener en cuenta

De este modo, los resultados finales tendrán más validez, ya que el tráfico ferroviario dado, según los planes de explotación previstos, estarán soportados por un análisis más robusto y cercano a la realidad. Si no se hubiera tenido en cuenta la señalización, es posible que los resultados obtenidos no estuvieran acorde a lo que luego se implantará, y por tanto las demandas de energía pudieran ser sensiblemente diferentes.

\section{Material móvil}

El material móvil es uno de los componentes más importantes a la hora de evaluar las posibilidades del dimensionamiento. La gran parte de la energía estará asignada a las necesidades de los trenes. El consumo de energía proviene de la tracción y de los sistemas auxiliares del tren (a/c, luces, sistemas de climatización, etc..). En cualquier estudio ambos han de ser tenidos en cuenta.

Mientras que los sistemas auxiliares, se suele estimar cuánto van a gastar eficazmente, para la necesidad de tracción hace falta un modelo mucho más complejo, y preferiblemente un modelo matemático ayudado por un simulador. Este simulador deberá tener las características básicas relacionadas con la tracción de cada tren, además del plan de explotación, geometría de la línea y tipo de señalización, limitaciones de velocidad estáticas, dinámicas. Teniendo todo conjuntado el tren deberá calcular la velocidad necesaria para cada instante de tiempo y en función de esa velocidad, calcular la potencia que demanda. La siguiente lista compone los datos principales para calcular la potencia:

- Curvas de tracción y frenado

- Rendimiento mecánico

- Curva de limitación de tracción por corriente máxima.

- Coeficiente de regenerabilidad

- Curva de regeneración eléctrica

- Masa

- Velocidad máxima

- Freno de emergencia y servicio 
- Potencia de auxiliares

- Coeficientes de resistencia al avance.

$\underline{\text { Sistema eléctrico }}$

El desarrollo de la explicación de elementos importantes para el diseño del sistema eléctrico finaliza en la descripción inicial de los elementos básicos que se deben tener en cuenta y que han de formar parte de cualquier propuesta de diseño eléctrico [18].

- Sistema de electrificación

- Catenaria/tercer carril

- Características de los conductores

- Sección

- Material (resistividad)

- Permeabilidad del terreno

- Conductancia carril-tierra cálculos de retorno

- Sistema de retorno y tomas a tierra

- Subestaciones

- Potencia de transformadores/rectificadores

- Número y localización

- Reversibilidad

- Cables conexión con red de tracción. Alimentadores.

- Autotransformadores

- Número y localización

- Supercondensadores

- Número y localización

○ Zonas neutras

- Localización

- Costes relacionados con la implantación

- Medioambientales

- Conexión a la red general de distribución

- Mantenimiento

- Instalación

- Frenado regenerativo 


\section{Bibliografía}

[1] M. C. Duffy, "George Stephenson and the introduction of rolled railway rail,» Journal of Mechanical Working Technology, vol. 5, no 3-4, pp. 309-342, 1981.

[2] O. Motoharu, K. Shunsaku y O. Hisao, "Japan's superconducting Maglev train,» IEEE Instrumentation \& Measurement Magazine, vol. 5, no 1, pp. 9-15, 2002.

[3] L. T. Rolt, «Thomas Newcomen: the prehistory of the steam engine.,» Dawlish: David and Charles, 1963.

[4] B. Spear, "James Watt: The steam engine and the commercializatino of patents.,» World Patent Information, vol. 30, no 1, pp. 53-58, 2008.

[5] F. Trevithick, Life of Richard Trevithick: With an account of this inventions, (Vol 1). E. \& FN Spon., 1872.

[6] E. L. Ahrons, The British steam railway locomotive, 1825-1925, Locomotive publishing Company limited, 1927.

[7] R. Diesel, «Internal combustion engine. U.S. Patent № 608,845. 9,» 1898.

[8] P. Ransome-Wallis, Illustrated Encyclopedia of World Railway Locomotives., Courier Corporation, 2012.

[9] P. Connor, London Underground Electric Train, Crowood, 2015.

[10] A. Steinmel, Electric traction-motive power and energy supply: basics and practical experience, Oldenbourg Industrieverlag, 2008.

[11] M. M. Maynar y F. J. Fernández, Ferrocarriles Metropolitanos: tranvías, metros ligeros y metros convencionales, 2002.

[12] F. J. Ontiveros, Transportes por ferrocarril. Estudios económicos, 1940.

[13] W. W. Hay, Railroad engineering, (Vol 1) John Wiley \& Sons, 1982.

[14] F. E. Al-Tony y A. Lashine, «Cost Benefit analysis of railway electrification case study for Cairo-Alexandria railway line.," Impact Assesment and Project Appraisal, vol. 18, no 4, pp. 323-333, 2000.

[15] INFRAS/IWW, «External costs of transport. Report commissioned by UIC,» ZurichKarlsruhe-Paris, 2000.

[16] J. Campos y G. De Rus, «Some stylized facts about high-speed rail: A review of HSR 
experiences around the world,» Transport Policy, vol. 16, no 1, pp. 19-28, 2009.

[17] U. P. d. C. CENIT (Center for Innovation in Transport, «Estimation des Resources et des Activités Economiques Lies a la grand Vitesse.,» UIC, Paris, 2005.

[18] F. Kiessling, R. Puschmann, A. Schmieder y E. Schneider, Contact Lines for Electric Railways. Planning, Design, Implementation, Maintenance, Siemens, 2009. 


\section{Sistemas de electrificación Ferroviaria. Elementos clave para distribución de energía y análisis de costes.}

En un sistema experto donde se integran diversas áreas del conocimiento relativas a sistemas ferroviarios, existen muchos componentes de los cuales se ha de tener una información específica. No es un objetivo de la Tesis explicar con detalle todos los actores que, de alguna manera u otra, intervienen en este Sistema experto. Aun así introducir al lector en la temática que se va a ir tratando, se ha considerado una tarea fundamental para situar el trabajo. Este Capítulo está pensado para aglutinar la descripción de componentes básicos.

El capítulo se divide en tres grandes apartados. El primer apartado va a servir para poner en contexto sobre qué sistemas de electrificación se suelen definir la elección de distribuir la energía. Los dos grandes bloques son sistemas de corriente continua y corriente alterna. Se comentarán los aspectos básicos de cada uno así como ventajas e inconvenientes de cada implantación en sistemas ferroviarios. A lo largo del segundo se van a introducir los elementos clave de los sistemas eléctricos que componen los sistemas comentados en el apartado primero. Estos elementos van a ser fundamentales en el transcurso del proyecto de esta Tesis. Finalmente el tercer apartado está pensado con la idea de dotar de contenido a las distintas zonas críticas de afectación a sistemas ferroviarios. La combinación de todos estos elementos y su implicación dentro del modelo, se detallarán en el Capítulo 4.

\subsection{Sistemas de tracción eléctrica.}

Hoy en día existen diversos tipos de electrificación para los sistemas distribución de energía para la tracción. Aunque actualmente el sistema de corriente alterna trifásica se emplea universalmente, el sistema de corriente continua se utiliza en determinados casos, tal y como los tiene clasificados Bhargava [1]:

- Corriente continua:

○ Alta tensión:3000V

○ Media tensión:1500V

- Baja tensión: de $600 \mathrm{~V}$ a $1400 \mathrm{~V}$ (incluye en particular la mayor parte de los transportes urbanos: tranvías, suburbanos)

\section{- Corriente alterna:}

- Frecuencia industrial: casi exclusivamente $25 \mathrm{kV}$, a $50 \mathrm{~Hz}$ ó $60 \mathrm{~Hz}$

- Baja frecuencia: principalmente $15 \mathrm{kV}$, a $162 / 3 \mathrm{~Hz}$.

La amplia gama de tipos de tracción actualmente utilizados en todo el mundo tiene sus raíces en la historia, en el progreso técnico y en los niveles relativos del desarrollo económico de los distintos países. 
Las opciones iniciales, atribuibles al estado de las técnicas en su momento, condujeron a la persistencia de modos de tracción que ya no eran óptimos, pues existía el deseo de normalizar los equipos.

El punto importante es que el motor de tracción más eficaz ha sido siempre el de corriente continua, por tanto el problema consiste en transformar la energía, que viene generalmente en forma de corriente alterna suministrada por la red de alta tensión, aplicándola [2]:

- o bien a las subestaciones, que la rectifican y reducen la tensión antes de pasarla al sistema de la catenaria (línea de contacto), de modo que se obtengan líneas de tracción de corriente continua (a $3000 \mathrm{~V}$, a $1500 \mathrm{~V}$, a $750 \mathrm{~V}$, etc)

- o bien a las propias locomotoras, de modo que la línea de tracción monofásica puede ser también de alta tensión. Esta técnica no pudo introducirse hasta mucho después de la tracción en corriente continua, cuando fue posible introducir rectificadores suficientemente eficaces que pudiesen estar instalados en las locomotoras.

Por consiguiente, un gran número de países poseen grandes redes electrificadas en corriente continua, que incluso han sido ampliadas por razones de uniformidad y conveniencias operacionales, a pesar de que actualmente lo normal sería que se utilizase la corriente industrial para corrientes de este tipo.

Veamos las ventajas e inconvenientes que nos proporciona la elección de cada una de estas alternativas de electrificación [3]:

\section{- Corriente continua}

- Ventajas:

- facilidad en la variación de la velocidad de los motores,

○ tensión de operación del motor baja.

- Inconvenientes:

- necesidad de reducir la distancia entre las subestaciones,

○ intensidad/sección elevadas en las líneas aéreas de contacto (LAC).

\section{- Corriente alterna}

- Ventajas:

○ tensiones altas, y de ahí, corrientes muy bajas (intensidad/sección baja)

○ catenarias más ligeras 
- subestaciones (transformadores y auxiliares) más sencillas y más espaciadas.

- Inconvenientes:

○ problemas de regulación de los motores,

○ equipo de a bordo más complejo

- en ocasiones, necesidad de conversión de las frecuencia de red (15 $\mathrm{kV}, 162 / 3)$. 


\subsubsection{Sistemas de corriente continua}

Los primeros sistemas de electrificación y hasta bien avanzado el siglo XX, tuvieron como modelo aquellos que funcionaban con corriente continua. Los motores de los trenes directamente trabajaban con la tracción proporcionada desde las subestaciones. En las subestaciones de tracción son necesarios los rectificadores para convertir la distribución trifásica de la red de distribución a tensión nominal de corriente continua.

Los sistemas de corriente continua no tienen una complejidad muy elevada. Una de las características principales es que los conductores de las catenarias suelen tener secciones más gruesas, para evitar en la medida de lo posible grandes caídas de tensión. Además las subestaciones de tracción suelen estar separadas por distancias cortas o muy cortas, debido a otro fenómeno eléctrico a controlar, las corrientes que circulan por los cables, y de ese modo evitar sobrecalentamientos. Obviamente, las pérdidas que se suceden a lo largo del flujo de corriente por los cables, también son altas. Por último otro de los fenómenos a tener en cuenta en sistemas de corriente continua, es la corrosión que se provoca debido a las corrientes de fuga en retorno. Como el retorno suele fluir por los carriles, si no hay feeder de retorno, esa corrosión puede perturbar gravemente con el paso del tiempo el material conductor, por lo que se aplican otro tipo de medidas para intentar subsanar en lo posible, como son instalación de feeder de retorno o instalar tramos equipotenciales entre los carriles para de esta manera mitigar el efecto de las corrientes de fuga.

La arquitectura básica de las catenarias, en función de las necesidades de los tramos a distribuir la tracción, se tienen en cuenta otros tipos de catenaria:

- Catenaria rígida: muy usado en sistemas de metropolitano. Como su nombre indica, a diferencia de la catenaria flexible, ésta se compone de una barra rígida y por tanto mucho más pesada. Para sostenerla necesita de unos soportes más robustos y menos separados

- Tercer carril: otro de los sistemas usados en diversos proyectos de corriente continua, donde la toma de corriente se sitúa a nivel de suelo (contacto carril-patín). Las tensiones de contacto y de paso se ven afectadas precisamente por la instalación del carril, y es uno de los factores importantes a calcular y poder así evitar daños a terceros, daños materiales debido a perturbaciones electromagnéticas como daños personales. 


\subsubsection{Sistemas de corriente alterna.}

La división entre sistemas de electrificación en 1x25 o también llamada monotensión, y sistemas dual/bitensión o 2×25 es lo suficientemente importante para que la explicación se separe en dos subapartados diferentes.

\subsubsection{Alimentación 1x25}

El sistema de electrificación 1x25 transporta la potencia necesaria para la tracción y la alimentación de elementos auxiliares asociados a la instalación manteniendo tensiones de $25 \mathrm{kV}$ entre hilo de contacto y retorno.

Las subestaciones utilizadas transforman las altas tensiones de la red pública a las de alimentación del sistema de tracción. La tensión de salida en los centros de transformación es $25 \mathrm{kV}$ (aunque en la práctica se alimenta a 27,5 kV).

Toda la corriente se distribuye a través de la línea aérea en contacto con el pantógrafo del tren. El retorno se realiza por los carriles y la tierra como vemos en la Figura 1:

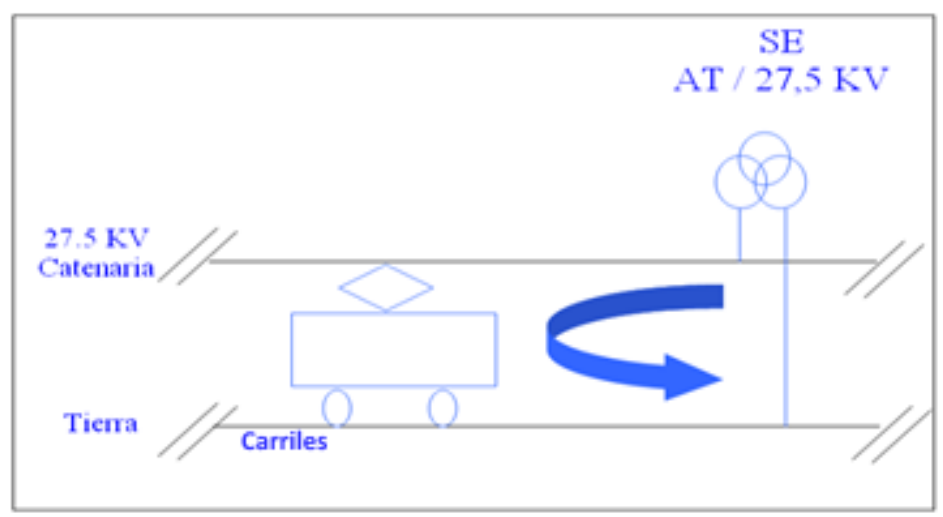

Figura 5 Alimentación 1×25

\subsubsection{Distribución de la corriente por los conductores}

La energía se distribuye desde las subestaciones hacia los trenes por medio de la catenaria. En el sistema de $1 \times 25$ se utiliza un sistema de alimentación simple con retorno por los carriles. En este sistema la corriente locomotriz tomada por el hilo de contacto vuelve a la fuente de alimentación a través del contacto de las ruedas con los carriles.

Como consecuencia de la fuga entre los carriles y tierra, cierta parte de la corriente deja el carril a lo largo de la denominada zona de efecto extremo. Para mejorar el efecto de apantallamiento y de esta forma disminuir las perturbaciones que genera el paso de corrientes tan elevadas por el retorno, la línea ferroviaria tiene un conductor de retorno auxiliar en paralelo con los carriles y soldado eléctricamente a éstos a intervalos regulares. Éste es un sistema simple con retorno por conductor y carriles (RC). Cuando el conductor de retorno tiene contacto metálico con los postes (y está conectado a los carriles a intervalos regulares, por ejemplo, cada 250 a 350 metros) se denomina hilo de 
puesta a tierra. Hay construcciones en las que el conductor de retorno está conectado al carril solamente cada 5 o $6 \mathrm{Km}$. Por lo tanto vemos que el sistema con conductor de retorno reduce la corriente de tierra y mejora el factor de apantallamiento en comparación con el sistema de retorno por los carriles.

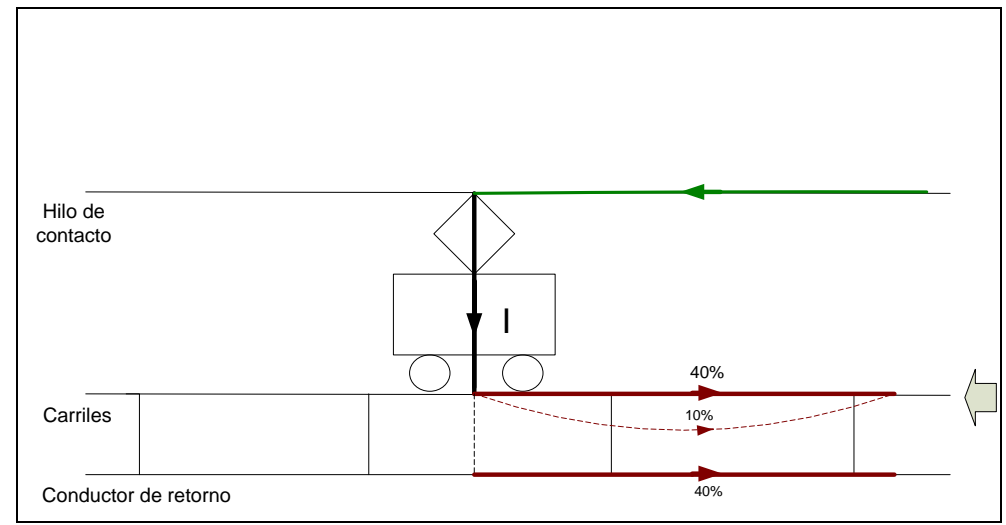

Figura 6 Reparto intensidades $1 \times 25$

La conexión desde los grupos transformadores de la subestación hacia la catenaria, suelen pertenecer a estos dos tipos:

\section{- Conexión en V}

Se realiza a base de un banco trifásico, mediante tres transformadores monofásicos conexión Dd, permitiendo la eliminación de un transformador.

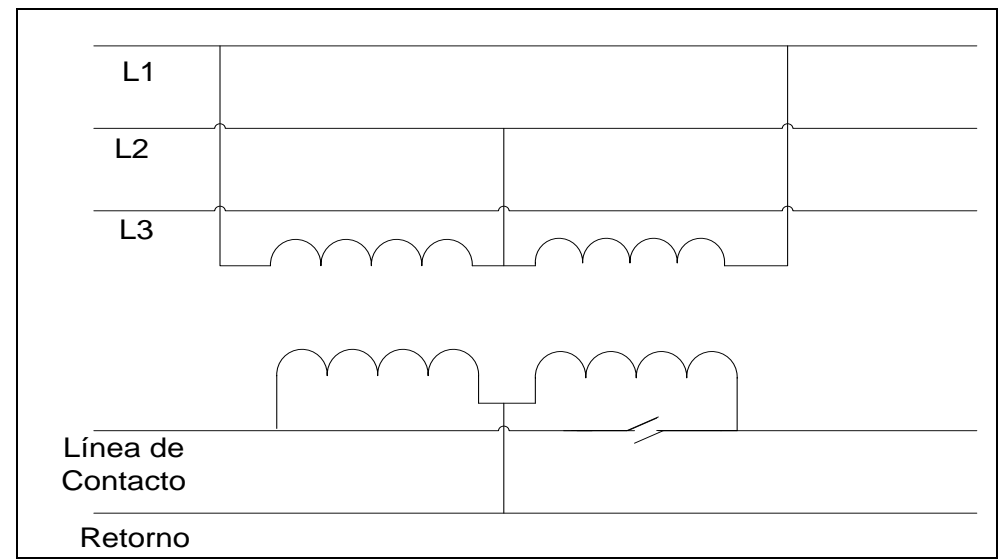

Figura 7 Subestación con conexión en V 


\section{- Conexión con transformadores trifásicos. Secundario en triángulo}

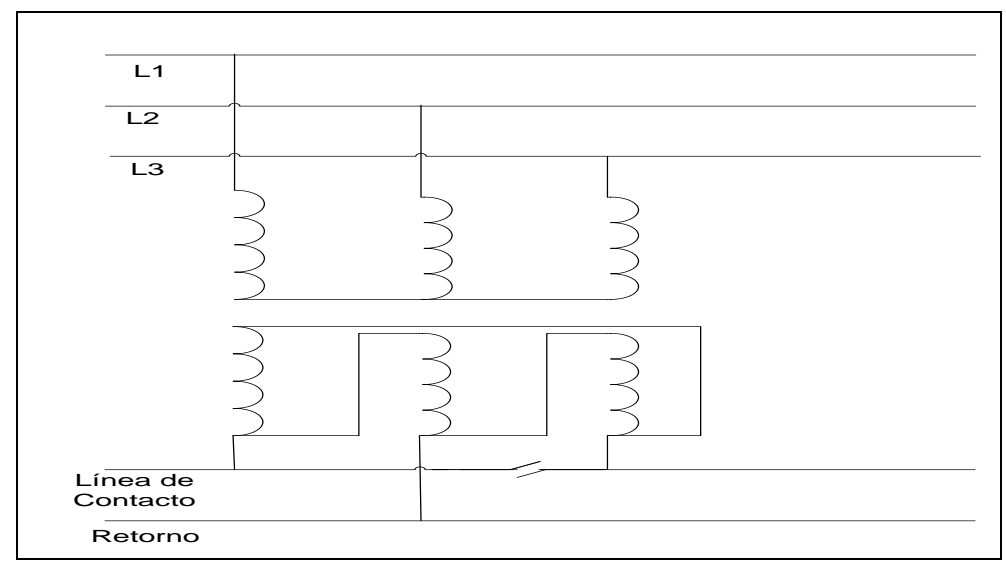

Figura 8 Conexión con transformadores en trifásico

En algunos casos, el aumento de la potencia de cortocircuito de redes de alta tensión permite prescindir de estos montajes y emplear subestaciones monofásicas ordinarias. Se podría incluso conectar las subestaciones sucesivas a las mismas fases, configuración que permitiría el acoplamiento de subestaciones en paralelo (ya que no se produciría cortocircuito). A pesar de esto, en todas las pruebas realizadas y que se mostrarán en el Capítulo 6 de esta Tesis, no se ha aplicado el acoplamiento de transformadores, siendo separados por zonas neutras.

A continuación se describe una subestación normalmente utilizada en alimentación $1 \times 25 \mathrm{KV}$, basado en las siguientes consideraciones:

- El mantenimiento de las instalaciones y una buena operatividad que hacen necesaria una división longitudinal de las líneas de contacto en tramos no limitados por el seccionamiento;

- la disminución de las caídas de tensión obtenida por la puesta en paralelo de las líneas de contacto de las dos vías.

Estas funciones de división longitudinal y de puesta en paralelo son realizadas por conmutadores instalados en las instalaciones de tracción:

- debido a la doble alimentación, asegura una reducción de las caídas de tensión;

- ofrece mayor posibilidad para la regulación de la subestación en caso de incidentes o bloqueos.

La alimentación bilateral, que permite que la corriente sea repartida entre dos subestaciones, hace posible limitar la corriente suministrada por cada fuente, y por tanto una reducción de los efectos perturbadores. 


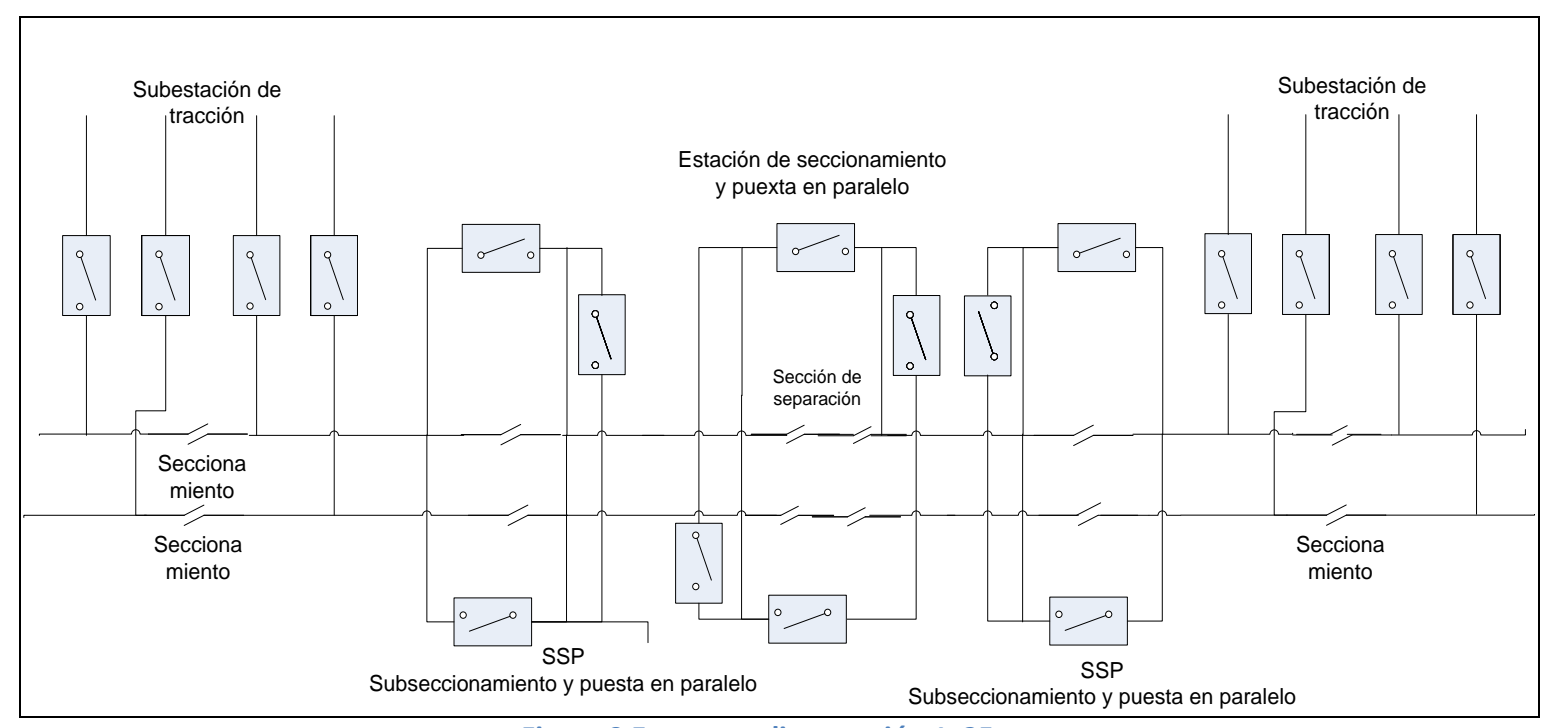

Figura 9 Esquema alimentación 1×25

\subsubsection{Alimentación $2 \times 25 \mathrm{kV}$}

El sistema de electrificación 2×25 transporta la potencia de tracción mediante un sistema de alimentación doble, es decir, se alimenta con una tensión que duplica la tensión normal de tracción $(25 \mathrm{kV})$.

Las subestaciones utilizadas transforman las altas tensiones de la red pública a las de alimentación del sistema de tracción. La tensión de salida en los centros de transformación es $55 \mathrm{kV}(2 \mathrm{X} 27,5 \mathrm{kV})$. La diferencia de potencial entre la catenaria y los carriles es la tensión de tracción $(27,5 \mathrm{kV})$. Por otra parte $27,5 \mathrm{kV}$ es la caída de tensión existente entre carril y el alimentador inverso (feeder negativo).

El sistema de alimentación 2x25 necesita auotransformadores [4], distribuidos a lo largo de la línea electrificada. Estos elementos tienen tensiones aplicadas totales de 55 $\mathrm{kV}$, induciéndose intensidades en los conductores conectados a ellos.

El uso de autotransformadores en la electrificación ferroviaria es debido a las ventajas que producen, siendo éstas:

- caídas de tensión más bajas en la línea de tracción;

- altas potencias;

- distancias mayores entre subestaciones,

todas estas características son óptimas para su instalación en zonas con una infraestructura de distribución de energía insuficiente.

Desde estos puntos de vista, representan un adelanto sobre los sistemas de alimentación en corriente alterna convencionales, aunque son más costosos. 


\subsubsection{Autotransformadores}

En este apartado se trata de mostrar más en detalle cómo interactúa el autotransformador en un sistema bitensión en ferrocarril. Tanto el principio básico del autotransformador, como la distribución de las corrientes inducidas por el mismo, se describen a continuación.

Los principios del sistema se resumen así:

- La corriente de retorno está limitada al alimentador invertido, salvo en la sección compensadora (entre dos autotransformadores consecutivos) cuando el tren está tomando corriente.

- Se aumenta la eficacia de la alimentación eléctrica (se indica en subestaciones en $2 \times 25$ )

Principio fundamental del autotransformador:

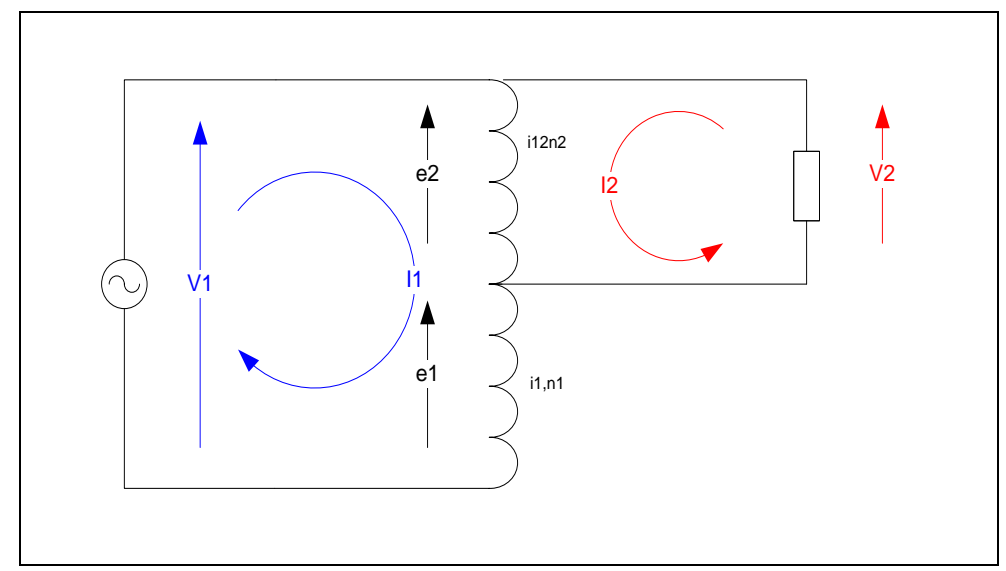

Figura 10 Principio fundamental del autotransformador

donde:

V1 tensión en los bornes del devanado primario del autransformador

V2 tensión en los bornes del devanado secundario del autransformador

I1 intensidad de entrada al devanado primario del autotransformador

I2 intensidad de salida del devanado secundario del autotransformador

n1, n2 número de vueltas del devanado en serie y del devanado común, respectivamente

e1, e2 fuerza electromotriz inducida en el devanado serie y en el común, respectivamente

i1, i2 intensidad en el devanado serie y el común, respectivamente 
Las f.e.m. inducidas e 1 y e 2 son proporcionales a los números de vueltas n 1 y n2:

$$
\frac{e_{1}}{n_{1}}=\frac{e_{2}}{n_{2}}
$$

Ecuación 1 Relación número espiras y f.e.m.

Si se trata de un autotransformador ideal en el que no haya pérdidas:

$$
\frac{V_{1}}{V_{2}}=\frac{e_{1}+e_{2}}{e_{2}} \frac{n_{1}+n_{2}}{n_{2}}
$$

Ecuación 2 Relación para autotransformador ideal

Si se aplican cargas al circuito y circula por éste una intensidad I2, la corriente del devanado primario aumenta hasta anular los flujos magnéticos causados por la corriente I2. Por tanto despreciando la corriente de magnetización, que es muy pequeña comparada con la corriente de carga, se puede escribir:

$$
\begin{aligned}
& I_{1}\left(n_{1}+n_{2}\right)=I_{2} n_{2} \\
& \frac{I_{1}}{I_{2}}=\frac{n_{2}}{n_{1}+n_{2}}
\end{aligned}
$$

Ecuación 3 Relación intensidades entre ambos devanados

Las relaciones de intensidades:

$$
\begin{aligned}
& i_{1}=I_{1} \\
& i_{2}=i_{1}-I_{2}=I_{1}-I_{2}
\end{aligned}
$$

Ecuación 4 Modelo ecuaciones intensidades

En este sistema de alimentación en el autotransformador, el número de vueltas del devanado en serie es generalmente igual al número de vueltas del devanado común.

$$
\begin{aligned}
& n_{1}=n_{2} \\
& \frac{I_{1}}{I_{2}}=\frac{1}{2}
\end{aligned}
$$

Ecuación 5 Relación intensidades en devanados teniendo en cuenta el número de vueltas

Llegamos a las siguientes relaciones:

$$
\begin{aligned}
& i_{1}=I_{1} \\
& i_{2}=-I_{1}
\end{aligned}
$$


Con la relación alcanzada en la Ecuación 6, se determina que las corrientes que circulan por el devanado serie y por el común son de igual intensidad pero de sentidos opuestos. El efecto succión de los autotransformadores indica que no circularía corriente por los carriles de la sección en ausencia de trenes.

Suponiendo un sistema ideal no habría circulación de corriente por el retorno como se observa en la siguiente Figura. También podemos estudiar detalladamente el reparto de intensidades que alimentan a los trenes en circulación.

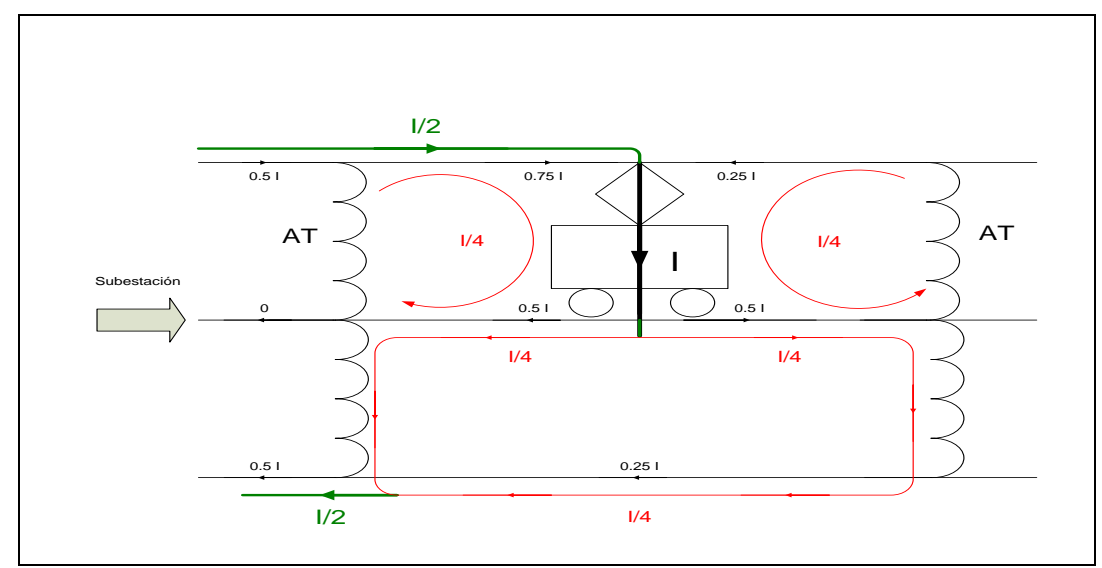

Figura 11 Reparto intensidades en sistema $2 \times 25$ en sección con tren circulando

El sistema no es perfecto, debido a la impedancia de fuga de los devanados de autotransformador y al hecho de que su potencia es limitada. En consecuencia, la corriente de tracción es suministrada también desde autotransformadores distantes. Por tanto circula corriente por los raíles en toda la sección del suministro eléctrico, como indica la línea a trazos en la Figura 8. Una parte de la corriente que circula por los raíles pasa a tierra; ésta es la corriente de retorno de tierra que puede causar problemas de inducción en las líneas de telecomunicación cercanas.

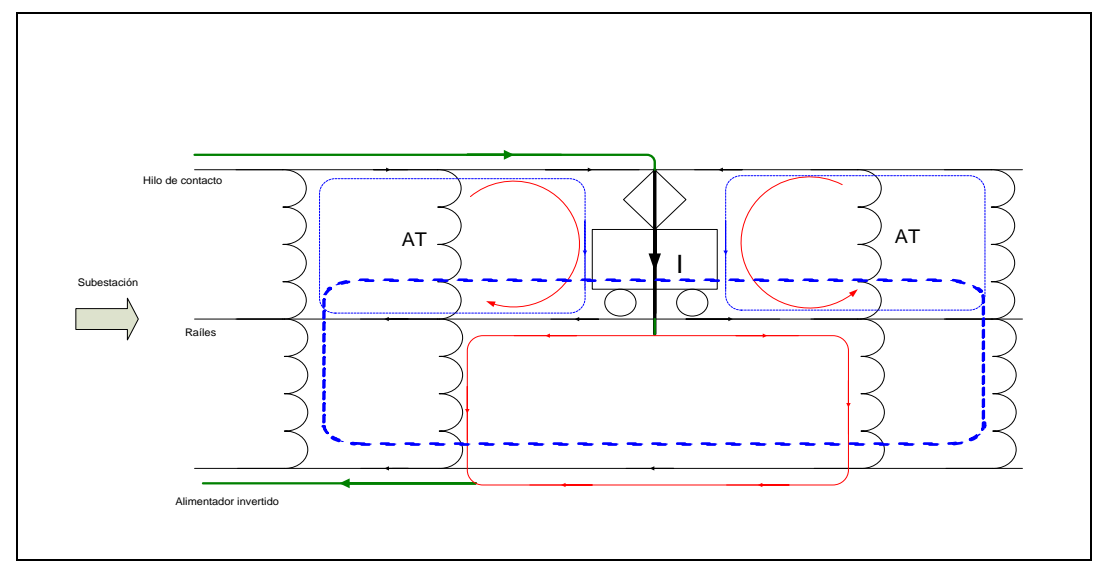

Figura 12 Reparto real intensidades 


\subsubsection{Subestaciones con autotransformadores ( 2 X $25 \mathrm{kV}$ )}

El transformador de la subestación tiene un secundario en el punto central definido para $55 \mathrm{kV}(2 \times 27,5 \mathrm{kV})$. La alimentación se efectúa entre la catenaria y el alimentador (feeder negativo) montado en los mismos postes, estando estos dos conductores en oposición de fase en relación con el punto central del transformador conectado a los raíles. Entre el alimentador y las líneas de contacto están enlazados los autotransformadores con una relación de transformación 55/27,5 kV instalados a intervalos regulares, estando su punto central conectado a los raíles.

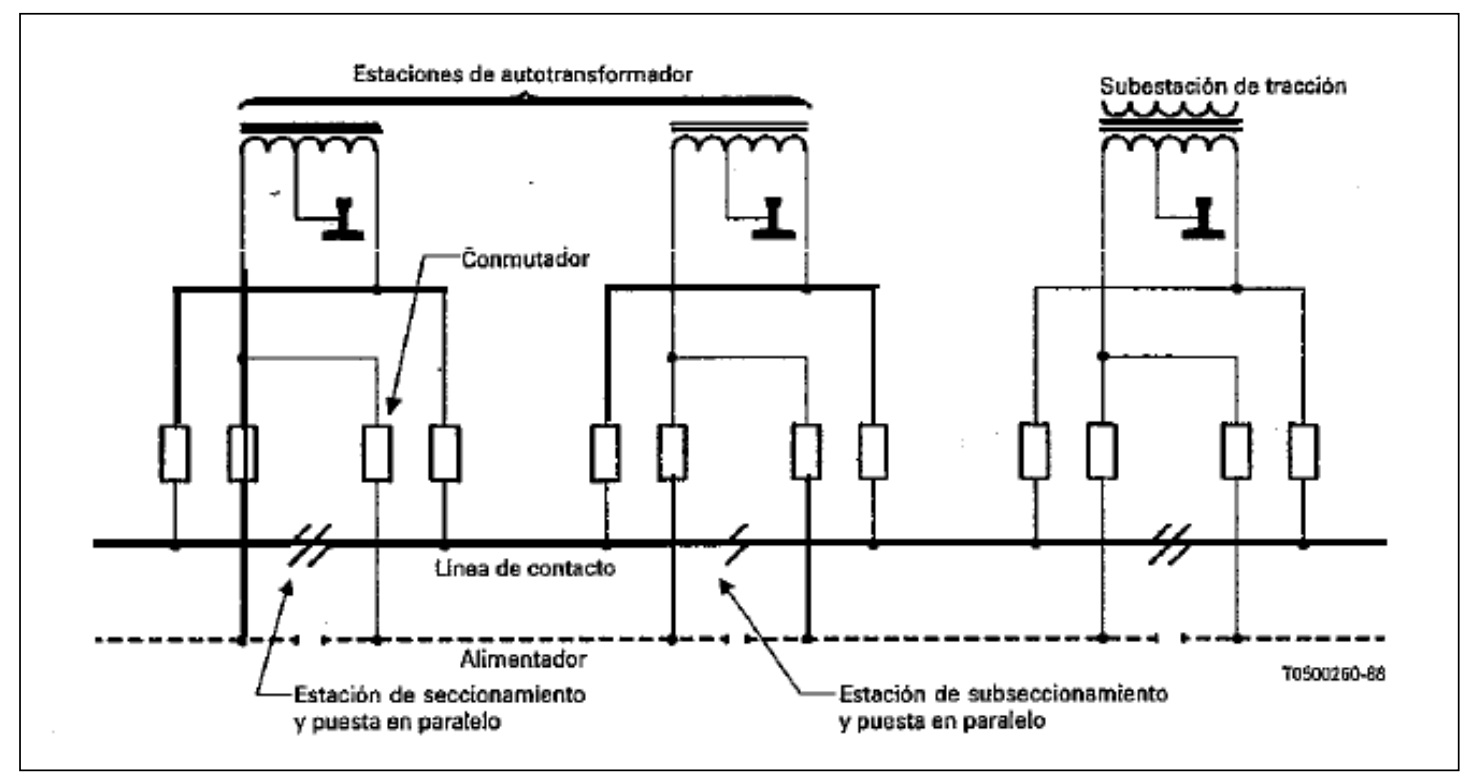

Figura 13 Esquema básico de una subestación de tracción en 2×25 


\subsection{Elementos básicos sistemas electrificación ferroviaria. Instalación.}

Los miembros que van a interactuar en el sistema eléctrico, tanto del simulador como en el cómputo de las ecuaciones del sistema experto son explicados a continuación.

\subsubsection{Catenaria.}

La composición clásica de la sección transversal es la que está implantada en el software Hamlet (Capítulo 3) y con la que el ingeniero puede trabajar:
○ Hilo de contacto
○ Hilo sustentador
- Hilo de retorno
- Feeder de refuerzo
- Feeder de retorno
o Carriles

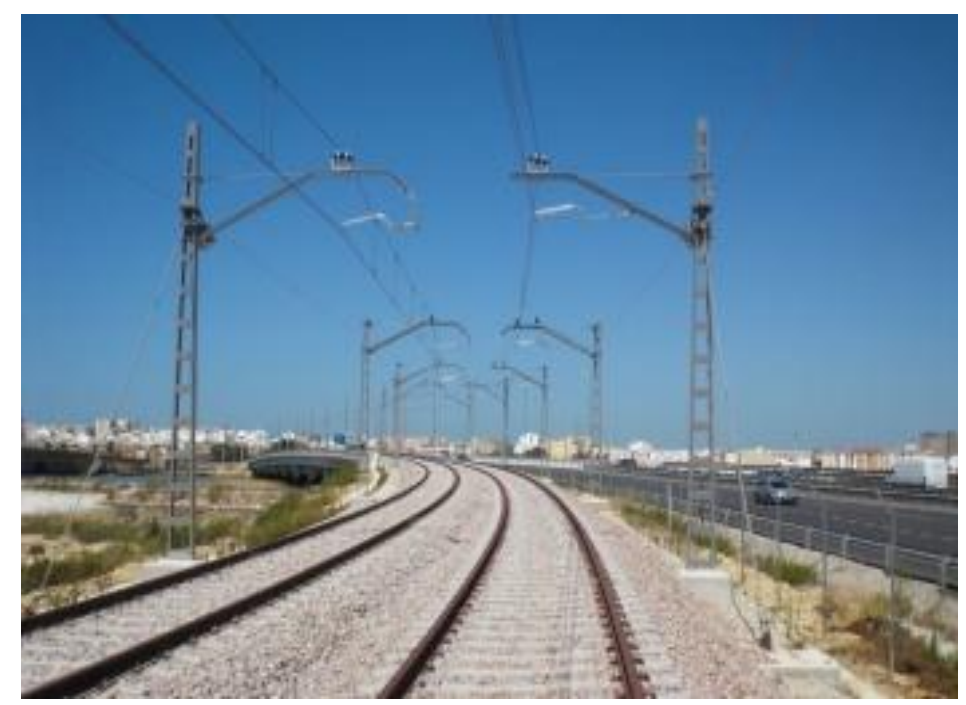

Figura 14 Ejemplo catenaria en vía ferroviaria

Es un problema recurrente la elección correcta del tipo de catenaria. Desde la configuración, si se ha de instalar tercer carril, catenaria rígida o áerea, tipo de material, si hace falta o no feederes de refuerzo o de retorno, los materiales de los carriles o de los cables. En definitiva muchas posibilidades que han de ser precisamente analizadas. También en función del coste de instalación, por lo que es un factor a incorporar en el algoritmo de este trabajo. La dualidad zona de instalación-catenaria a instalar es un factor determinante en las decisiones de los ingenieros, aunque en muchos casos viene determinado por los estudios realizados para la instalación de la línea, sobre todo cuando no se tienen que realizar obras mayores como soterramientos, túneles o viaductos. En estos últimos casos, la elección de la catenaria conlleva un estudio más pormenorizado. 


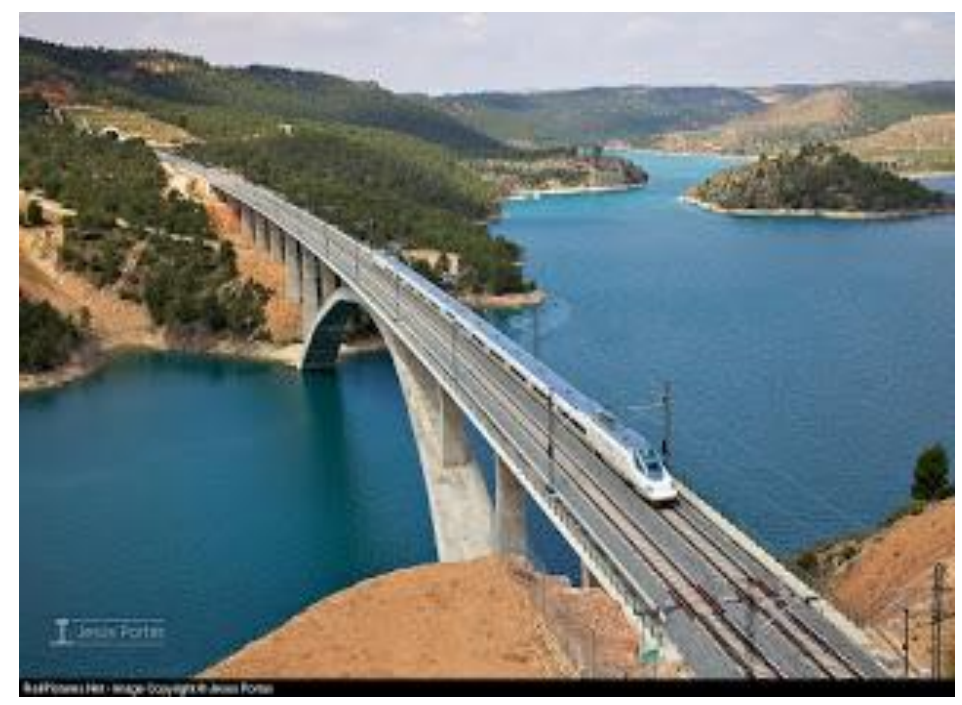

Figura 15 AVE Madrid-Valencia. Viaducto de Contreras. Foto: Jesús Portas

\subsubsection{Subestaciones}

Las subestaciones de tracción, también referidas con su acrónimo SET, cumplen un cometido esencial en la transmisión de energía a los trenes. En la fase de diseño de la SET es muy importante calcular la cantidad y composición de las mismas, en cuanto a grupos de potencia, en función de análisis previos, resultados obtenidos de simulaciones del sistema al que van a dar soporte y también, en función del presupuesto que se maneje. El coste de una subestación de tracción es muy alto, por lo que es importante acertar en la decisión final.

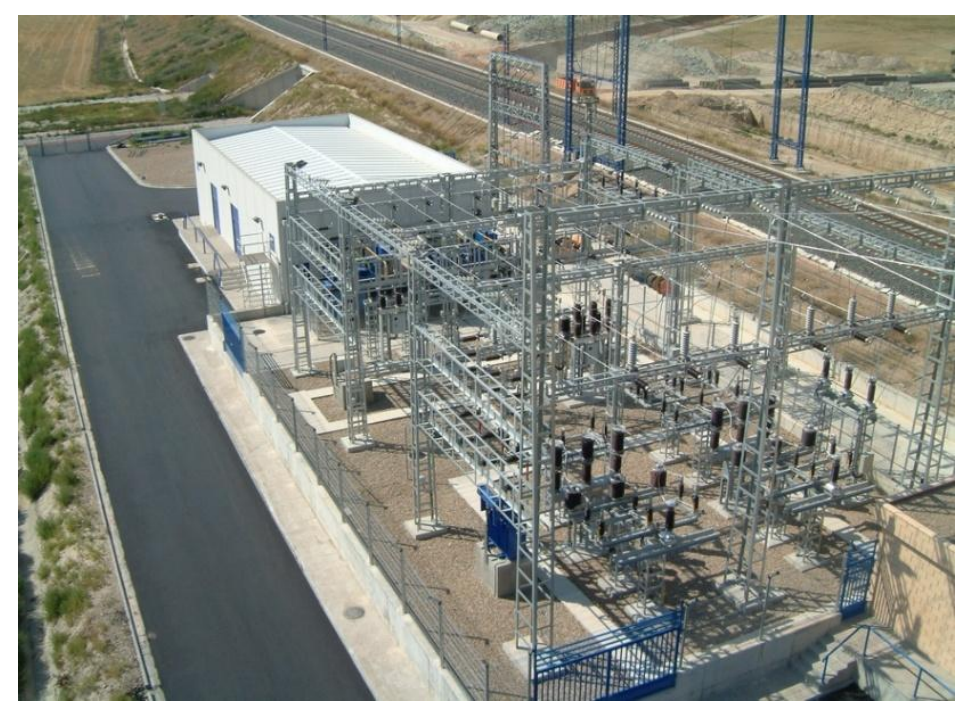

Figura 16 Ejemplo Subestación de tracción (FUENTE: Electrén "Subestaciones de tracción")

Ese coste es muy alto no tan solo por los elementos que la componen, sino por la obra civil que conllevan. No es lo mismo la instalación bajo tierra para un sistema metropolitano, que en exterior; o también en una zona donde ya hay una obra civil bajo tierra como por ejemplo en una estación de metro o instalar donde no la hay. Finalmente otro ejemplo estaría reflejado en la tesitura de tener que instalar en una zona de difícil 
acceso, solo porque los análisis previos lo exigen porque con cálculos a priori, no ven otra alternativa. En definitiva en la fase de diseño de instalación de una subestación se han de tener en cuenta factores como:

- Instalación de todos los elementos diseñados para el correcto funcionamiento de la transmisión de energía desde la red general a la catenaria.

- La instalación de sistemas auxiliares para dar soporte energético.

- Sistemas de protección de la subestación. Concretamente es necesario incorporar un sistema de protección para aquellas subestaciones que usan transformadores de potencia y otro protector para la catenaria.

- Sistema de control y de adquisición de datos

- Edificación principal y secundaria. La construcción de la subestación estará situada dentro de compartimentos edificados, los cuales, dependiendo del diseño final y de las características de la línea, tendrá unos requerimientos específicos a nivel geológico, de conexión con la red general y catenaria, medioambiental, etc.

Un ejemplo lo encontramos en una subestación de tracción instalada para una línea ferroviaria próxima a Logroño, cuyas características principales serían:

- Soterramiento de la construcción

○ Tensión nominal de $66 \mathrm{kV}$, con tres grupos transformadores

- Dos conexiones de toma de la red general

- Protecciones de transformador y elementos auxiliares situadas en celdas blindadas

○ Construcción de un pórtico de feeders con seis salidas hacia la catenaria.

El coste presupuestado fue de 3.9 millones de euros y un plazo de 12 meses.

\subsubsection{Autotransformadores}

En los sistemas con electrificación dual en alterna, los autotransformadores son una pieza básica en el dimensionamiento eléctrico, ya que permiten duplicar la tensión y que se transmita por el feeder negativo de la sección transversal instalada, facilitando así el flujo de corriente por la catenaria, y evitando problemas en los conductores debido a la baja intensidad de corriente que suelen tener los sistemas dual. 


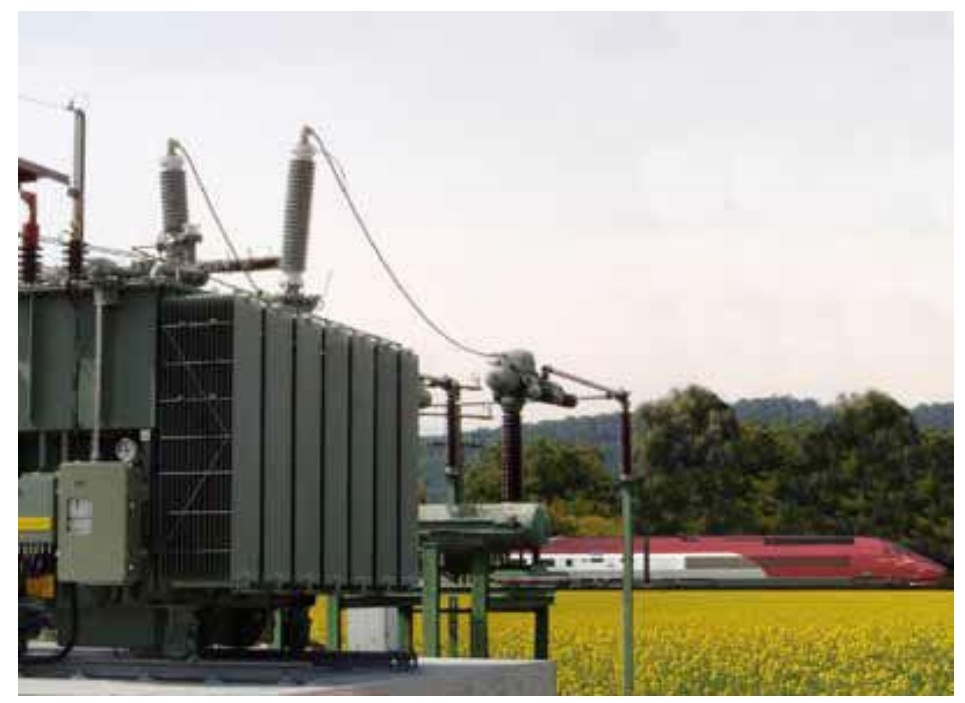

Figura 17 Ejemplo Autotransformador situado a lo largo de línea ferroviaria

Entre la catenaria y el autotransformador también es necesaria la instalación de cableado de apoyo, de protección y del feeder negativo. Para minimizar la impedancia del cableado, es necesario acortar la distancia entre la catenaria y el autotransformador [5]. Los cables de soporte han de conectarse entre los autotransformadores y la catenaria también, por lo que en los costes de instalación deben ser tenidos en cuenta, y sobre todo en qué zonas se va a querer instalar este componente para los sistemas dual en alterna. En el apartado 2.1 correspondiente al sistema eléctrico $2 \times 25 \mathrm{kV}$, se explica con más detalle la participación de estos elementos, y se define la importancia y funcionamiento dentro del sistema en el que se instala.

\subsubsection{Zonas Neutras}

Necesarias para separar zonas eléctricas adyacentes. Cuando el sistema de electrificación es usando corriente alterna, es necesario instalar estos separadores para evitar desequilibrios entre zonas eléctricas distintas, debido a la conexión entre distintas fases, provenientes de subestaciones contiguas. Otros motivos son para separar entre sistemas de electrificación distintos, por ejemplo entre DC $3 \mathrm{kV}$ y AC $15 \mathrm{kV} 16,7 \mathrm{~Hz}$, en líneas donde es necesario disponer de distintos sistemas.

Hay dos variantes para construir las separaciones eléctricas, cuya idea es que los pantógrafos de un mismo tren, que son aquellos situados en los coches para los tipos de trenes pertenecientes a la flota en la explotación ferroviaria determinada, no estén a la vez en ambos sistemas eléctricos:

- Que la separación sea menor que la distancia menor calculada entre los pantógrafos de los trenes en operación. Necesitan de equipos aislantes y una sección intermedia de toma a tierra. Además también necesitan seccionadores para el paso de un sistema a otro de las tomas de contacto del tren-catenaria. 
- Que la separación corresponda a una distancia mayor que la mayor calculada entre los pantógrafos extremos de los trenes en operación.

Existen diversas medidas de seguridad que se tienen en cuenta, como que al pasar por las zonas neutras se bajan los pantógrafos, o bien instalación de sistemas auxiliares de transmisión de energía durante ese tramo, conjuntamente con un contacto a tierra, para evitar así algún posible fallo y sobre todo las consecuencias de alimentación para la tracción.

La instalación de zonas neutras deben evitar zonas tales como:

- En las inmediaciones de señales o agujas.

- Curvas que debido a las características de construcción, tienen una limitación de velocidad muy alta.

- Rampas con gradientes elevados, con alta demanda de tracción puntual.

\subsubsection{Seccionadores y enlaces}

Ambos componentes facilitan y flexibilizan la configuración del SET. Los seccionadores, al igual que se usan en las zonas neutras, facilitan el aislamiento de dos zonas eléctricas contiguas por motivos de seguridad o para solventar algún tipo de situación degradada que haya ocurrido. Los enlaces eléctricos, por ejemplo hacen referencia a los cables alimentadores que conectan los bornes de las subestaciones de tracción con la catenaria. Al igual que las decisiones que se deben tomar con el cableado de la catenaria confrontando calidad/servicio, esta disyuntiva sería trasladable a la utilización o no de estos elementos de conexión. 


\subsection{Zonas de especial interés para la evaluación de sistemas eléctricos ferroviarios}

El sistema experto pretende aunar el esfuerzo de encontrar un diseño válido para el sistema eléctrico de energía, junto el impacto que puede llegar a tener respecto ciertos condicionantes. En los siguientes apartados se describe cuáles son los entornos elegidos para examinar su trascendencia dentro de un diseño de sistema eléctrico, y se trata de apuntar la importancia que efectivamente reciben en el sector ferroviario.

\subsubsection{Mantenimiento}

Los sistemas ferroviarios deben tener un plan de mantenimiento con el objetivo de evitar fallos por el desgaste propio del tiempo o del uso. Los elementos instalados que pertenecen al sistema de aporte de energía también tienen una serie de acciones que están dirigidas a aumentar la vida funcional del sistema y a subsanar posibles deficiencias que provoquen un fallo que deje sin alimentación a un tramo de la línea durante un periodo de tiempo, o incluso a toda la línea.

En el apartado que trata el Plan de Mantenimiento [6] de las primeras acciones que se tienen en cuenta, es que el plan deberá ser convenientemente descrito y detallado por la entidad contratante, y entre las características específicas que deben estar:

- Rutinas de mantenimiento de subestaciones y puestos.

- Registro de condiciones, resultados y experiencia adquirida

- Valores límite de seguridad para establecer límite de velocidad de los trenes

- Frecuencia de comprobación y tolerancias aplicadas

- Medidas adoptadas en caso que se superen los valores previstos.

La misma forma de proceder se aplica a la línea aérea de contacto. Es una de las prerrogativas que se deben formalizar en las primeras etapas de un proyecto ferroviario. Las tareas de mantenimiento deben cuidar la estabilidad del sistema y no degradar la seguridad del mismo.

En la norma EN 13306 [7], se establecen las distintas clases de mantenimiento:

- Métodos de corte: donde los componentes se van a reemplazar por otros, por lo que se necesitaría redundancia en los elementos a cambiar para evitar largos lapsos de tiempo con el sistema en fallo.

- Métodos rutinarios: donde lo que se persigue es evitar fallos inesperados en el sistema, en este caso de energía.

Adaptado al tipo de diseño que obtiene el sistema experto, se podría diferenciar claramente de realizar tareas de mantenimiento a elementos tales como subestaciones, autotransformadores, zonas neutras y por otra parte a la catenaria. 
En [8], libro dedicado prácticamente en exclusiva a la explicación de todo lo relacionado con el sistema de energía ferroviario, se relatan 4 tareas principales a efectuar en catenarias, y en general, del sistema de energía:

- Mejorar la fiabilidad

- Realizar diagnósticos periódicos del sistema

- Mantenimiento correctivo

- Reposición de elementos

Planificar el mantenimiento es una tarea por tanto que necesita un estudio previo por las consecuencias que puede acarrear las malas decisiones. Existen una serie de trabajos [9] [10] [11] cuyo planteamiento se basa en estudiar la influencia de una mala planificación de los trabajos de mantenimiento, efectuados en diversos elementos de la toma de energía, y cómo afectan al ciclo vital y también a la operación ferroviaria. Por ello, los autores creen que es muy importante una correcta planificación de las tareas y tratan de optimizar el proceso y sus resultados. Otros enfoques [12], tratan el problema del correcto mantenimiento, pero ya teniendo la idea de que el método de aplicación que proponen, su finalidad es ayudar a los ingenieros a que el diseño no se aparte de la calidad necesaria de las tareas de fiabilidad del sistema.

El trabajo que se lleva a cabo en las subestaciones que se usan para la tracción [13], tiene un alto grado de implicación en los costes finales, tanto directos como indirectos. En este artículo tratan de aplicar un algoritmo de optimización bi-objetivo, con la idea que los elementos que están involucrados en una subestación, se pueda llegar a identificar qué tipo de mantenimiento deben tener, cuándo realizarlo y qué metas se pretende conseguir. Con todo ello y una vez más, para que afecte lo mínimo posible el desgaste de la subestación, sobre todo teniendo en cuenta que es una de las partes más importantes del sistema de energía.

La evaluación de costes de mantenimiento es una tarea que no suele ser objetiva, ya que no es fácilmente medible debido al gran número de variables, entre ellas el tiempo global de vida útil y el uso que se haga de las instalaciones, que hace de este trabajo muy complejo de querer discretizar un valor de coste. El planteamiento es que en función de la experiencia del diseñador y de traslaciones que pueda realizar a cada proyecto respecto de otros ya trabajados, sea capaz de asignar costes a las etapas de mantenimiento ya comentadas, como son asignar un cierta fiabilidad, tareas de diagnóstico, mantenimiento correctivo y tareas de reposición. Pero además de poder asociar ciertos niveles de coste a las tareas mencionadas, algunas zonas muy específicas pueden estar sujetas a altos valores de costes relacionados con el mantenimiento.

En la práctica, cuando se realiza cualquier tarea de mantenimiento a lo largo de la línea se ha de contar con que a la zona donde se va a trabajar, se deberá tener preparado: 
- Herramientas y equipamiento especial

- Vehículos especiales

- Equipamiento para medidas y diagnóstico

Estos tres condicionantes aumentan la complejidad dependiendo de la zona donde se va a ejecutar estos trabajos, e incluso puede no ser viable, por el tamaño considerable de los vehículos de mantenimiento (Figura 14), o al plantear dificultades tales que se tenga que interrumpir la operación durante un determinado periodo. Si para alguna zona en concreto se prevé que puede ser muy alto el coste por una correcta aplicación del mantenimiento, es importante que el sistema experto sea capaz de intentar evitar esta zona, o al menos evitar la instalación de elementos de administración de potencia. La evaluación debe contemplar que aunque la catenaria debe pasar por esa zona, pueden existir diversos tipos de catenaria de modo que según la calidad de los materiales, hagan que el ciclo de vida del cableado sea un factor a tener en cuenta, además del desgaste que puede producir el plan de explotación pensado.

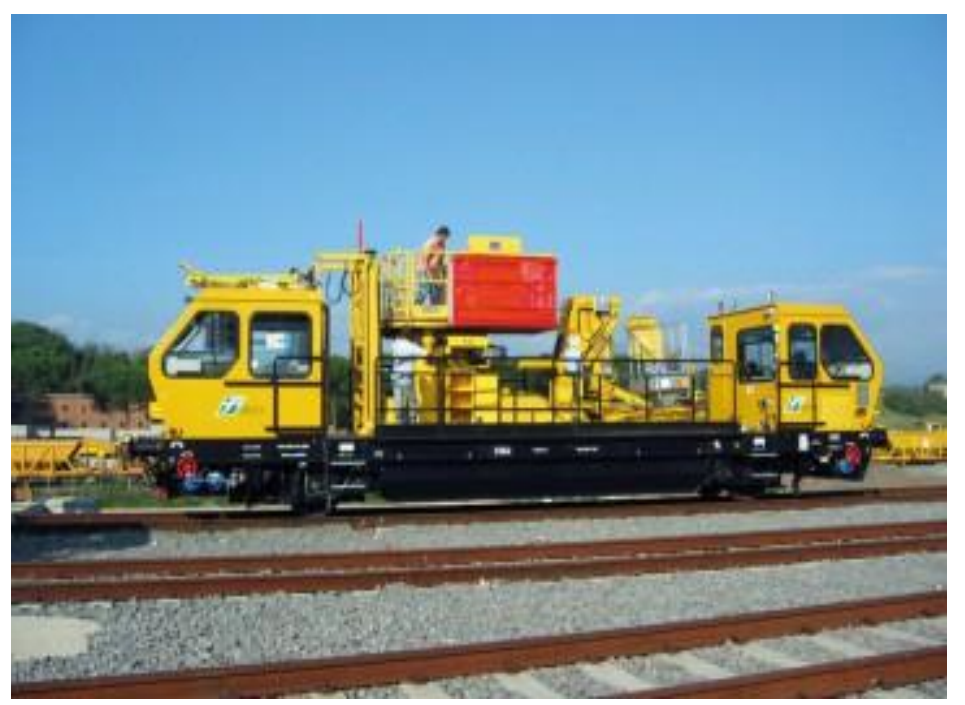

Figura 18 Ejemplo maquinaria para instalación-mantenimiento de catenaria (Fuente Página web Tesmec)

Conjuntamente, los costes de mantenimiento asociados a los elementos de transformación (subestación, autotransformadores, zonas neutras), también pueden influir zonalmente, puesto que el traslado y realización de las tareas, son factores que aumentan/disminuyen el cómputo global del trabajo.

\subsubsection{Conexión con la red general}

Las subestaciones de tracción suelen estar formadas por grupos transformadores o rectificadores de la media/alta tensión proveniente de la red general de distribución. Esta red de distribución suelen estar construidas y en funcionamiento para propósitos anteriores a los relativos a la tracción ferroviaria. Para poder trabajar, las subestaciones de tracción necesitan conectarse a una acometida de la red general.

Algunos trabajos tratan de optimizar el camino que debería recorrer la infraestructura de energía hasta poder realizar la conexión con la red general de 
distribución. La idea que se plantea en este trabajo es que la flexibilidad de la conexión, no se va a plantear desde un punto de vista bidireccional, sino que se va a dar la posibilidad de establecer una serie de zonas o áreas críticas, pertenecientes a la nueva infraestructura ferroviaria, de modo que el sistema experto tenga la capacidad de intentar evitar esas zonas, y por tanto recomendando otras, ya sea para la instalación de subestaciones, autotransformadores o cualquier elemento necesario para la instalación del sistema de aporte de energía.

\subsubsection{Medioambiental}

No solo la conciencia social incide en salvaguardar lo mejor posible la integridad del medio ambiente, sino que directivas europeas (directiva 96/48/CE) y normativas así lo exigen como requisito esencial a la hora de poner en marcha un proyecto ferroviario. Esta importancia intrínseca al diseño de cada proyecto, la hemos querido trasladar al sistema experto. El diseñador debería conocer los efectos que puede producir una línea con graves afectaciones medioambientales.

\begin{tabular}{|c|c|c|c|c|}
\hline Propiedad & Unidades & Coche & Tren & Avión \\
\hline Requerimiento energía específica & $\mathrm{kWh} / 100 * \mathrm{Pkm}^{1}$ & 48,7 & 10,3 & 62,8 \\
\hline Emisiones de $\mathrm{CO}_{2}$ & $\mathrm{~kg} / 100 * \mathrm{Pkm}$ & 12,29 & 4,75 & 17 \\
\hline Emisiones de $\mathrm{NO}_{\mathrm{x}}$ & $\mathrm{g} / 100 * P k m$ & 133 & 3,8 & 88 \\
\hline Emisiones de CO & $\mathrm{g} / 100 * P k m$ & 209 & 0 & 20 \\
\hline Emisiones de Hidrocarburos & $\mathrm{g} / 100 * P \mathrm{~km}$ & 27 & 0 & 8 \\
\hline Emisiones de hollín & $\mathrm{g} / 100 * P \mathrm{~km}$ & 0 & 1 & \\
\hline $\begin{array}{l}\text { Para nuevas construcciones: } \\
\text { requerimiento aéreo para } \\
\text { proyecto de similar dimensión }\end{array}$ & $\%$ & 285 & 100 & 170 \\
\hline $\begin{array}{l}\text { Niveles de ruido a } 25 \mathrm{~m} \text { de } \\
\text { distancia }\end{array}$ & $\mathrm{dB}(\mathrm{A})$ & 73 & 92 & \\
\hline
\end{tabular}

Tabla 6 Comparativa de afectación medioambiental por parte de medios de transporte

Existen muchos trabajos que han tratado de medir las bondades de la tracción eléctrica comparándolos con otros sistemas de transporte, que de forma directa, usan materiales fósiles para generar movimiento y por tanto, más emisiones de $\mathrm{CO}_{2}$ (ver Tabla 1). Esto favorece la implantación de los ferrocarriles, pero aún así, es necesario considerar aspectos tales como:

\section{- Protección de entornos de considerable nivel ecológico}

Siempre es importante evitar una zona de alto nivel ecológico por razones obvias. El proyecto de obra civil ya debería evitar cualquier zona de este tipo, pero al avanzar en el proyecto e incluir la infraestructura correspondiente al sistema de energía, se han de tener en cuenta áreas más grandes y que probablemente no se sepan hasta qué punto son accesibles o no hasta que se pueda calcular o simular el comportamiento de los trenes y saber si es posible

\footnotetext{
${ }^{1} 100 * \mathrm{Pkm}$ quiere decir que está comparado con 1 persona caminando $100 \mathrm{~km}$
} 
evitar dicha zona. Esto es debido a que por ejemplo, por caída de tensión debido a la exigencia de la operación por las cercanías de la zona crítica, se demanda más potencia desde la línea, y por tanto sería un tramo a evitar por temas de consumo de energía, pero sería aceptable por no violar ninguna zona medioambiental o protegida. U otro caso se puede plantear cuando se quiere pasar por una zona de alto valor ecológico, pero el daño colateral medioambiental no sería a nivel superficial, sino por las aves que componen la fauna de la zona y por la afectación hacia ellas.

- Uso de superficies grandes de tierra

En este caso, cuando la obra civil y el sistema de energía han sido construidos sobre un terreno, es difícil que en un largo periodo de tiempo, ese terreno y sus cercanías, puedan ser usados para otro motivo. Los movimientos de tierra excavados, transportados, cimentaciones, etc, no pueden no ser tenidos en cuenta en la cuantificación del impacto final del proyecto.

\section{- Impacto estético de la nueva infraestructura}

No se puede medir subjetivamente el impacto que puede tener la construcción de una subestación de tracción, o la conexión entre la red de tracción y distribución, pero el ingeniero si que puede conocer los entornos en los cuales ese impacto puede ser especialmente dañino. Se trata como siempre de en la medida que sea posible, evitar estos efectos colaterales.

\section{- Impacto de corrientes inducidas}

En sistemas con corriente alterna de alta tensión, como ejemplo los trenes de alta velocidad en España que funcionan con tensión $2 \times 25 \mathrm{kV}$ a $50 \mathrm{~Hz}$, se han de tener en cuenta que los campos electromagnéticos que se generan en la cercanía de los sistemas de energía, hacen que la actividad humana esté muy limitada, y en concreto el uso de aparatos eléctricos, por lo que se deben evitar.

En el documento de normativa para Victoria Rail sobre construcción de subestaciones [14], se listan las acciones que se deben evitar para no comprometer los mínimos tolerables para la afectación a sistemas medioambientales protegidos:

a) No se deberían eliminar árboles, arbustos ni vegetación fuera del perímetro permitido.

b) Afectación a áreas de cultivo, deberían ser minimizadas.

c) Cualquier área de cultivo afectada, debería ser de alguna manera repuesta en su condición inicial o mejorada

d) Donde se haya excavado, debería reusarse como Relleno especial.

e) El sobrante de tierra excavada debería integrarse perfectamente en el medio que se va a posar. 
f) Los residuos contaminantes deberían ser tratados con los requerimientos de la EPA 448.

g) El control de la erosión del terreno y los sedimentos, se deberá diseñar en la fase inicial del proyecto. 


\section{Bibliografía}

[1] B. Barghava, «Railway Electrification Systems and Configurations,» de Power Engineering Society Summer Meeting, 1999.

[2] A. Ogunsola y A. Mariscotti, Electromagnetic Compatibility in Railways: Analysis and Management, Springer Science \& Business Media, 2012.

[3] A. Steinmel, Electric traction-motive power and energy supply: basics and practical experience, Oldenbourg Industrieverlag, 2008.

[4] C. Courtois, "Why the $2 \times 25 \mathrm{kV}$ alternative?[autotransformer traction supply].," de IEE Colloquium on IET 50kV Autotransformer Traction Supply Systems-the French Experience, , 1993.

[5] R. J. Hill, «Electric railway traction. Part 3: Traction power supplies, » Power Engineering Journal, vol. 8, no 6, pp. 275-286, 1994.

[6] P. Europeo, Especificación Técnica de Interoperabilidad del sistema ferroviario transeuropeo convencional. Subsistema de Energía, 2001.

[7] E. CEN, «Maintenance Terminology. 13306,» 2001.

[8] F. Kiessling, R. Puschmann, A. Schmieder y E. Schneider, Contact lines for Electric Railways. Planning, design, Implementation, Maintenance, 2009.

[9] T. K. Ho, Y. L. Chi, L. Ferreira, K. K. Leung y L. K. Siu, «Evaluation of maintenance schedules on railway traction power systems," Proceedings of the Institution of Mechanical Engineers, Part F: Journal of Rail and Rapid Transit, vol. 220, no 2, pp. 91-102, 2006.

[10] A. L. Zorita, O. Duque, M. A. Fernández y L. A. García-Escudero, «Determination and optimization of the maintenance frequencies in the overhead contact line system," Journal of Transportation Engineering, vol. 136, no 11, pp. 964-972, 2010.

[11] T. Lidén, Survey of railway maintenance activities from a planning perspective and literature review concerning the use of mathematical algorithms for solving such planning and scheduling problems, 2014.

[12] S. Chen y T. Ho, "Maintenance schedule optimisation for a railway power supply system,» International Journal of Production Research, vol. 51, no 16, pp. 4896-4910, 2013.

[13] S. K. Chen, T. K. Ho, B. H. Mao y Y. Bai, «A bi-objective maintenance scheduling for power feeding substations in electrified railways,» Transportation Research Part C: Emerging Technologies, vol. 44, pp. 350-362, 2014. 
[14] V. R. I. O. G. S. V. 010.4, Railway traction substation construction Standard. 


\section{Herramienta software de simulación ferroviaria. Hamlet.}

Un simulador permite realizar estudios, análisis y recoger resultados de un sistema complejo sin tener que usar directamente el sistema real. Esto permite moldear el sistema simulado para recrear distintas situaciones, que si se estuviera trabajando con el sistema real, tendrían un coste muy elevado. En el caso concreto de simuladores ferroviarios, el coste de hacer pruebas durante la operación real de un sistema ferroviario, sería prácticamente inasumible, tanto a nivel económico, operación de la línea ferroviaria, en gestión de tiempos o en disposición de recursos humanos.

El desarrollo de potentes simuladores ferroviarios está amparado por la creciente evolución de la potencia de los ordenadores. Las mejoras de la potencia que pueden llevar a cabo, incide en los análisis que se pueden acometer con los simuladores.

Las empresas y centros de investigación relacionados con el mundo ferroviario, en el que su negocio se base o bien en simuladores de conducción, o bien en consultorías de comportamiento ferroviario, ya sea dinámico, eléctrico u operacional, no pueden abstraerse de desarrollar constantemente estas herramientas informáticas. Para nuestras investigaciones y más en concreto, para nuestra metodología, no se podía atacar el problema sin plantearnos el desarrollo de un simulador para el análisis, que llegara a ser lo más completo posible. El simulador ferroviario Hamlet (Herramienta de aplicación multidisciplinar para líneas eléctricas de trenes), aglutina los módulos y funcionalidades que se buscaban para realizar nuestras investigaciones.

El simulador Hamlet permite estudiar un sistema ferroviario analizando su explotación, comportamiento eléctrico, conflictos y optimizaciones posibles mediante la simulación de todos sus componentes.

La alta capacidad de los simuladores para apoyar estudios en sistemas ferroviarios, ha hecho que empresas y entidades investigadoras se hayan planteado su desarrollo.

- Silvia [1] desarrollado para el estudio del dimensionamiento eléctrico ferroviario y para apoyar a análisis de optimizaciones. Con este simulador se ha probado y trabajado sobre todo en sistemas de corriente alterna y explotaciones de alta velocidad

- Rsim [2] realizado dentro del ámbito de una Tesis Doctoral, está enfocado a la modelización del sistema ferroviario desde el punto de vista del sistema eléctrico. La modelización propuesta es tanto para sistema global como para partes fijas. Incluye estudios de regeneración de energía.

- SimuX [3] Aparece en diversos trabajos publicados para optimización del consumo de energía, de modo que el simulador es el encargado de obtener los datos de tiempos de viaje y demandas de potencias para que el algoritmo de 
optimización halle la combinación óptima de modos de conducción para el objetivo planteado.

- Opentrack [4] Completo simulador desarrollado en la ETH de Zürich. Está basado en técnicas de programación orientada a objetos y los datos están almacenados en RailML, de modo que permite al usuario una rápida adaptación a sistemas ferroviarios nuevos, y un trasvase de datos cómodo y eficiente. Este simulador está pensado sobre todo para cálculos de optimización y diseño de los planes de explotación ferroviario.

- Train Power System Simulator (TPSS). El autor [5] se planteó el desarrollo de este simulador como base para sus análisis de la Tesis Doctoral. Los autores necesitaban el simulador para obtener datos de los flujos de carga que se producían durante los planes de explotación de las líneas en estudio, y de esta manera poder entrenar la red neuronal que pretendía optimizar el sistema de electrificación.

- RailSys [6] es usado por los autores para encontrar y realizar pruebas de un algoritmo para la estabilidad horaria de los planes de explotación ferroviarios. Este simulador tiene muy avanzado el módulo del que hacen uso los autores, de modo que la integración del tren/trenes, junto con el diseño de la geometría e infraestructura de la línea y finalmente la inclusión del sistema de señalización, hace que el análisis sea bastante realista.

- Otros simuladores comerciales son TrackFeed [3], el Symtrack o SytrasSyditrac. Por supuesto cada uno de ellos tiene sus características propias, pero en general todos tratan de obtener resultados concluyentes de las simulaciones pertenecientes a líneas ferroviarias estudiadas.

Es evidente que cada simulador ferroviario tiene sus características propias que hacen que sea más efectivo para un tipo de trabajo concreto, o que tengan una precisión mayor gracias al modelo matemático escogido o capacidad computacional de los algoritmos de cálculo integrados en ellos. A pesar de todas las similitudes que puedan tener, aun no se tiene una normativa relativa a comprobar la fiabilidad de los simuladores ferroviarios. El ámbito y alcance de esta normativa está siendo estudiada y se prevé que se llegue a consensuar una, que permita verificar la validez de los simuladores ferroviarios. Esta normativa prevé obtener una serie de requisitos que los simuladores ferroviarios deberán cumplir. Aparte de esta normativa, si que se puede hablar de aspectos comunes entre los simuladores. Todos ellos deben tener capacidad para trabajar y modelar con las siguientes áreas:

- Infraestructura. Soporte de bajo nivel donde los demás elementos de más alto nivel van a ir instalándose y adecuándose a la configuración inicial. 
- Señalización. Los movimientos de los trenes están sujetos a la regulación propia del sistema. Es obligatorio un sistema de señalización si se quiere tener un simulador mínimamente flexible y potente.

- Material rodante. Un tren es un sistema muy complejo y por tanto en este módulo es muy frecuente poner límites al modelo que se va a trasladar finalmente al simulador.

- Sistema eléctrico. Es el sistema de dimensionamiento energético común, y al igual que para los módulos de señalización y material rodante, dependerá de la profundidad y precisión que se quiera alcanzar para tener un sistema más o menos adaptable a la realidad que se pretende estudiar.

A continuación se van a explicar los detalles más significativos de los módulos desarrollados para el simulador Hamlet.

\subsection{Diseño}

La fase inicial de trabajo para simular una línea ferroviaria consiste en el estudio de los planos pertenecientes al proyecto. En los planos se buscan datos acerca de señalización, alzado, radios de curvatura, posición de las estaciones, agujas, breteles. El planteamiento inicial es que todos estos elementos van a tener que interactuar durante los trabajos del simulador.

En los siguientes apartados se va a describir cómo se ha conformado la estructura del simulador y el alcance de cada módulo.

\subsubsection{Infraestructura}

Es el módulo que da soporte y funcionalidad a los elementos de la línea y del sistema de señalización. Uno de los aspectos importantes a tener en cuenta es que dependiendo del sistema de señalización, la distribución de elementos va a ser completamente distinta. Debido a esta particularidad, se planteó esta fase inicial. La idea era dotar al simulador de una gran flexibilidad para posteriores implantaciones sobre la capa inicial.

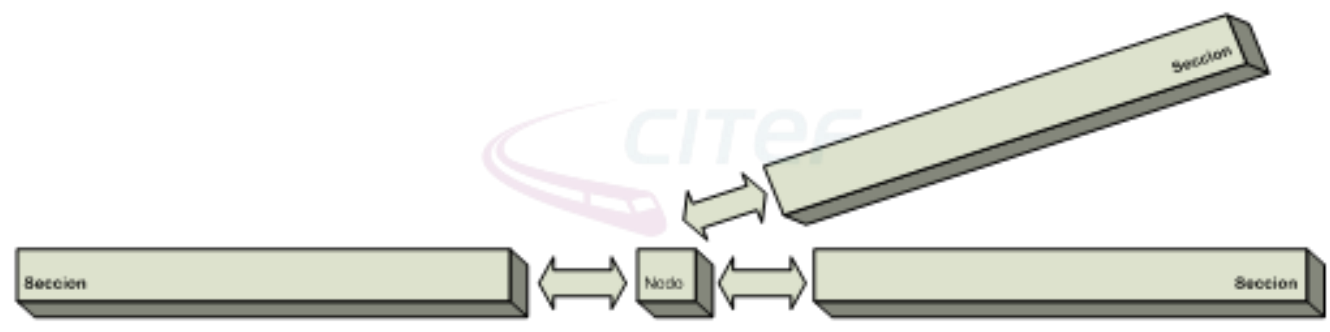

Figura 19 Esquema elementos básicos infraestructura

La formación básica para la infraestructura, como se puede observar en la figura anterior, estaría formada por secciones y nodos. 
Las secciones contienen la información relativa a la geometría de la línea que afecta al tren (pendientes, radios de curvatura, etc.). En cuanto a los nodos, cada uno de ellos representa el punto donde las vías posicionan agujas para el cambio de vías.

Usando este núcleo de configuración, se tiene la capacidad de situar dentro del mismo los elementos de los sistemas de señalización, como pueden ser los circuitos de vía, señales, agujas, breteles.

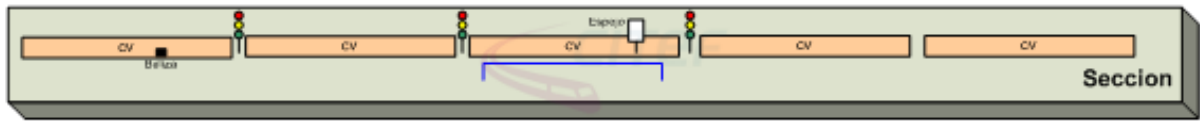

Figura 20 Esquema básico configuración Sección geométrica

\subsubsection{Señalización}

Este módulo se encarga de simular el funcionamiento de distintos niveles de señalización (ERTMS, CBTC, etc.), determinando el comportamiento de sus elementos (enclavamientos, señales, balizas, circuitos de vía, etc.) y la manera en que gobiernan la circulación de los trenes en cada escenario.

Cabe destacar que en el proceso de evolución de los sistemas de señalización desarrollados e integrados en el Hamlet, ha sido necesaria la implantación de la posibilidad de incluir Enclavamientos [7]. Los sistemas ferroviarios actuales necesitan de un sistema de control que proteja a los trenes que circulan por la línea de los posibles percances a los que se puede enfrentar. Estos accidentes que se tienen que evitar, y por tanto permitir circulaciones seguras, serían descarrilamientos debido a agujas en un estado distinto al que el tren requeriría, choques contra toperas y otro caso a evitar son las colisiones entre trenes. Los autores del artículo [7] se plantean como objetivo el conseguir validar un enclavamiento creado para un sistema informático, en definitiva cómo integrar un enclavamiento en un simulador ferroviario. Recogiendo esta idea, en nuestro simulador, el enclavamiento en si podría ser un módulo aparte, ya que depende de la complejidad con la que se quiera trabajar. A día de hoy, el enclavamiento permite:

- Inclusión de elementos tales como señales y balizas de posicionamiento.

- Detección de incompatibilidad entre itinerarios

- Establecimiento/desestablecimiento de itinerarios

- Normalización de itinerarios a paso de tren

Una de las claves del método que permite simular el comportamiento del Enclavamiento es un sistema de Reglas. Estas reglas de comportamiento se han de diseñar teniendo en cuenta el Enclavamiento, de modo que sea consecuente con las acciones que permita o no.

Evidentemente no siempre es necesario para los resultados que se desean la recreación del enclavamiento. Dependerá del análisis final que se persiga y si el sistema 
de señalización hace necesario imperiosamente, el estudio e implantación del enclavamiento.

Los sistemas de señalización que se han implementado han sido CBTC [8] y los niveles 1 y 2 de ERTMS [9].

Para el caso ERTMS nivel 1, el Movement Authority queda determinado a partir de la posición del tren y el estado de las señales, que son manejadas por un enclavamiento simulado. La comunicación entre el tren y el sistema de señalización tiene lugar cuando el tren pasa sobre las balizas situadas en puntos determinados de la línea. La señalización lateral fija deja de ser relevante para la conducción del tren.

Para el caso ERTMS nivel 2, el Movement Authority queda determinado a partir de la posición del tren y del primer circuito de vía ocupado, aguja en movimiento o a la contra o topera según el sentido de avance del tren; además, si en el escenario hay señales presentes, como en el caso anterior, un enclavamiento simulado se encarga de manejarlas. La ocupación y liberación de los circuitos de vía se determina según el número de ejes que haya dentro de ellos, teniendo en cuenta también el retraso que hay entre que sale un tren y el RBC (Radio Block Center) autoriza a que el siguiente pueda entrar. La comunicación entre el RBC y los trenes se considera continua.

En el caso de CBTC, el Movement Authority queda determinado a partir de la posición del tren y del primer obstáculo según el sentido de avance del tren. Estos obstáculos pueden ser otro tren, una aguja que no esté en el estado adecuado o una topera. La comunicación entre el sistema de control y los trenes son continuas.

\subsubsection{Tren}

El módulo del tren está dividido en varias partes, cada una de las cuales se ocupa de una serie de tareas:

- $\quad$ TrenLinea engloba las comunicaciones entre el tren y la infraestructura.

- ControlConduccion recoge los elementos necesarios para responder a los distintos modos de conducción y para controlar las paradas.

- ControlTraccion proporciona las consignas de velocidad, par, intensidad, freno y aceleración que se adecúan al modo de conducción, tipo de tren y características de la geometría.

- Dinámica realiza los cálculos dinámicos del avance del tren. 


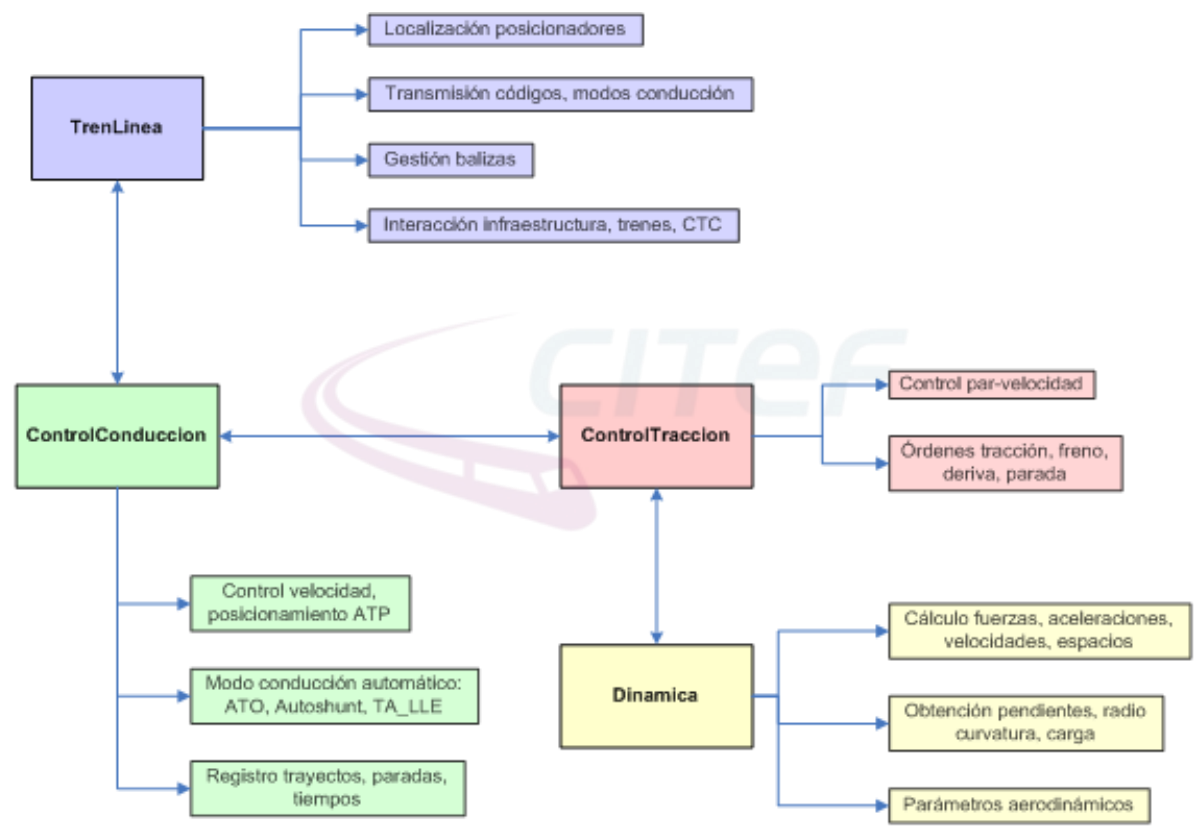

Figura 21 Diagrama modular funcionamiento material rodante

\subsubsection{Eléctrico}

El modelo y algoritmo eléctrico están pensados para poder simular cualquier tipo de configuración, ya sea para corriente continua como alterna, en configuración 1x25 ó $2 \times 25$ y cualquier tensión nominal.

El módulo tiene como objetivo configurar el escenario eléctrico en el algoritmo de cálculo. Para conseguirlo, se utilizan una serie de elementos, que se pueden clasificar en los siguientes tipos:

- Elementos geométricos, para definir la malla eléctrica.

- Componentes eléctricos, que modelan subestaciones, autotransformadores, cargas estáticas, seccionadores, etc.

- Elementos de relación, que se utilizan para definir las relaciones existentes entre los componentes eléctricos y la malla eléctrica.

- Componentes de la línea eléctrica, que definen la configuración y propiedades de los conductores que forman el sistema de alimentación.

\subsection{Metodología}

Para construir el sistema ferroviario que se va a simular, se define la geometría y disposición de la red, las características del sistema de señalización y del sistema de alimentación eléctrica. Después, se define el plan que indica cómo se va a realizar la explotación. Cada uno de los trenes que recorren los itinerarios puede poseer unas características propias, como las características mecánicas de tren, las paradas a realizar, la duración de la maniobra, su nivel de prioridad, sus limitaciones de velocidad, etc. De 
ahora en adelante, nos referiremos a cada uno de estos trenes que recorre un itinerario como circulación.

Posteriormente, se realiza la simulación del escenario, calculando en cada instante las velocidades, tiempos y potencias demandadas, y detectando los conflictos que surjan entre las circulaciones.

Además de este estudio mecánico de la red, en cada ciclo de simulación se realizan los cálculos eléctricos, que incluyen consumos, caídas de tensión y pérdidas en la línea, tensión en pantógrafo, intensidades que circulan por las catenarias, raíles y feeder, potencia suministrada por las subestaciones y autotransformadores, etc.

De esta manera, partiendo de una composición de tráfico definida y conociendo las características de diseño (intensidades máximas admisibles, tensiones mínimas admisibles, potencias nominales de los autotransformadores, potencia nominal de los transformadores de la subestación de tracción, etc.), se pueden realizar simulaciones en condiciones normales y en condiciones degradadas (pérdida de autotransformadores, pérdida de subestaciones, etc.) y así conocer los parámetros para diseñar la red, y ver, sin necesidad de construirla, cómo sería su comportamiento en cada caso.

La herramienta de simulación permite al usuario definir y simular cualquier tipo de situación para cualquier disposición de trenes y elementos de que consta. El circuito y su configuración son completamente dinámicos, y el algoritmo de resolución será distinto en cada caso. Así, la aplicación es capaz de adaptarse a cualquier tipología de líneas y circuitos eléctricos.

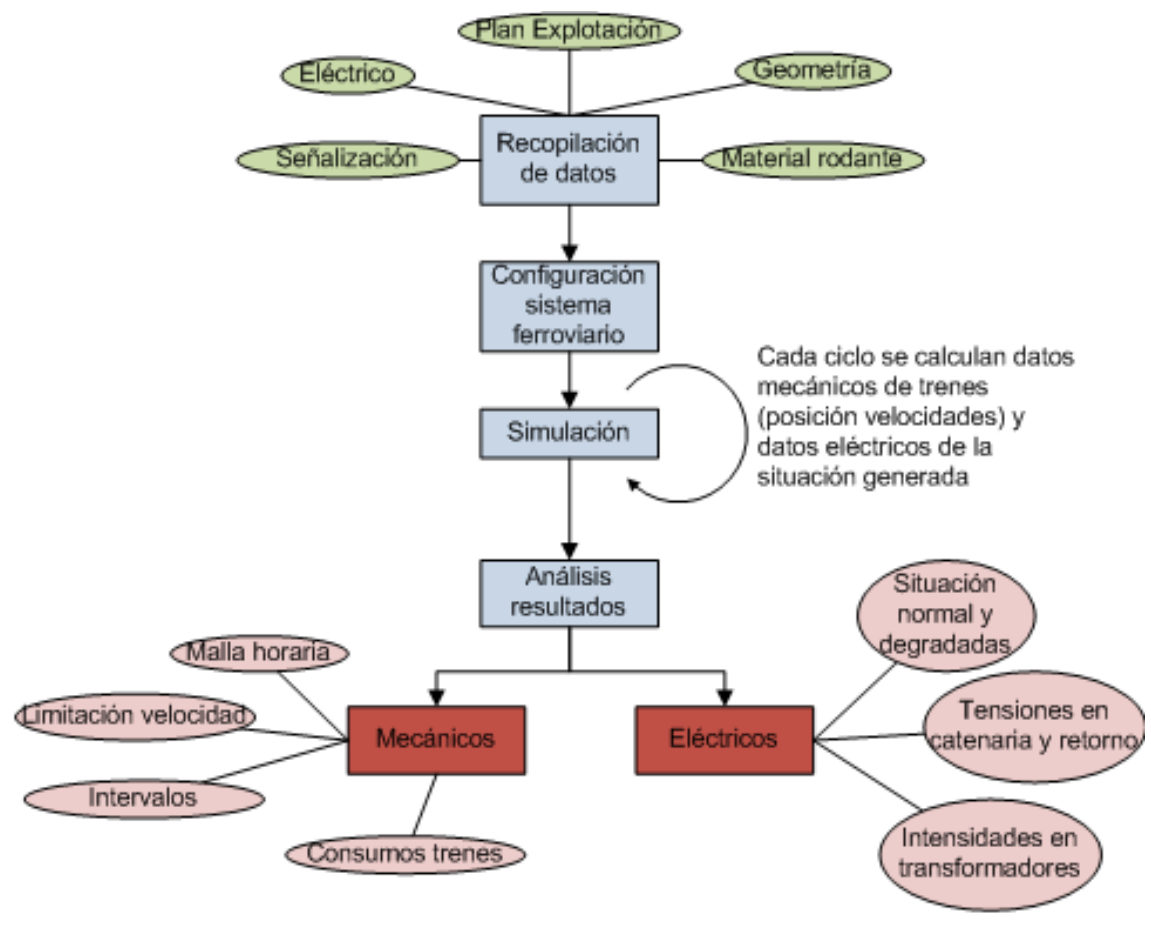

Figura 22 Diagrama Metodología de trabajo usando Hamlet 


\subsection{Datos iniciales}

Como datos de entrada al simulador se introducen:

- Características mecánicas y eléctricas del material rodante.

- Características del trazado: planta, alzado, perfil estático de velocidades, etc.

- Características de la línea: número de vías, estaciones y apartaderos.

- Características de la explotación: plan de explotación, tiempo de parada en cada estación, itinerario de las circulaciones, etc.

- Características del sistema de electrificación: configuración del sistema de alimentación (línea aérea de contacto, tercer riel, etc.), posición de los elementos del sistema de electrificación: subestaciones, autotransformadores (si los hubiera), zonas neutras (si las hubiera), etc.

- Características del sistema de señalización: tipo de señalización, enclavamientos, señales, balizas, etc. 


\subsection{Cálculos mecánicos}

Se basa en calcular el avance de cada uno de los trenes, analizando las fuerzas que actúan sobre ellos en cada ciclo de simulación y el comportamiento de deben seguir según las condiciones de explotación y las consignas dadas por el sistema de señalización.

En primer lugar, se determina cuál va a ser el comportamiento de cada tren en ese instante, es decir, si va a acelerar, derivar o frenar. Para ello, dos son los factores que se estudian:

- Estado del sistema de señalización, dado por la simulación de los elementos que lo forman. La herramienta permite simular diferentes sistemas, como son CBTC y ERTMS. Estos sistemas se han integrado en el Hamlet, teniendo en cuenta el comportamiento del tren en función de cada uno, sin llegar a la simulación completa de los sistemas de señalización.

- Paradas que tenga que realizar el tren, según el plan de explotación que se haya definido.

Después, se analizan las fuerzas que actúan sobre el tren y según su comportamiento se calcula la fuerza de tracción o de frenado que tiene que aplicar. Se realiza un balance de fuerzas para calcular la aceleración que se produce. A partir de esta aceleración, se calcula la nueva velocidad del tren y cuánto ha avanzado.

A partir de la posición y potencia demandada por todos los trenes que están circulando por la línea, se establece la comunicación con el módulo eléctrico al cual se le pasa esta información para que pueda realizar los cálculos necesarios para comprobar el estado del sistema eléctrico. Esta comunicación directa entre los módulos permite que, por ejemplo, en caso que el módulo eléctrico compruebe que la intensidad de corriente en el punto de conexión tren-catenaria supere el límite establecido, se le comunique al tren para que pueda adaptar su velocidad al límite marcado por las características del tren.

Los resultados de los cálculos se van guardando para que al finalizar la simulación puedan consultarse y procesarse para detectar conflictos y proponer soluciones y optimizaciones al diseño del sistema ferroviario. 


\subsubsection{Análisis de fuerzas que actúan sobre el tren}

Las fuerzas que se consideran que actúan sobre el tren son las siguientes [10]:

- Resistencia al avance.

- Acción de las curvas.

- Acción gravitatoria.

- Fuerza de tracción o frenado.

\subsubsection{Resistencia al avance}

Esta fuerza de resistencia al avance engloba las resistencias mecánicas y resistencia aerodinámicas mediante la siguiente fórmula, que se basa en el modelo planteado por Davis [11]:

$$
R_{a v}=A+B v+C v^{2}
$$

Ecuación 1. Cálculo de resistencias al avance del tren

donde

$\mathrm{R}_{\mathrm{av}}$ es la resistencia al avance y se suele expresar en daN

A, B y C son valores dependientes de la aerodinámica de cada material rodante y se suelen medir en daN, daN $/(\mathrm{km} / \mathrm{h}) \mathrm{y}$ daN $/(\mathrm{km} / \mathrm{h})^{2}$ respectivamente.

v es la velocidad del tren en $\mathrm{km} / \mathrm{h}$

\subsubsection{Resistencia en túnel}

Cuando un tren circula por dentro de un túnel se ve afectado por la resistencia debida a la compresión del aire entre el tren y las paredes del túnel. La resistencia depende en gran medida a la sección del túnel, de la longitud y sección del tren. Se suele incorporar a la suma de resistencias por medio de una constante $\mathrm{k}_{\mathrm{t}}$. De este modo quedaría la ecuación:

$$
R_{a v}=k_{t}\left(A+B v+C v^{2}\right)
$$

Ecuación 2. Aplicación de la constante de efecto túnel a resistencias al avance

\subsubsection{Acción de las curvas}

El valor de la resistencia debida a las curvas se calcula a partir del coeficiente de resistencia en curva que se haya definido para el tren y del trazado.

$$
R_{a c}=m \frac{K_{c}}{r}
$$


donde

m es la masa del tren en toneladas $(\mathrm{t})$

$\mathrm{K}_{\mathrm{c}}$ es un coeficiente que suele ser 500/600 para vehículos de pasajeros y 800 para mercancías

$r$ es el radio de la curva en metros $m$

\subsubsection{Acción gravitatoria}

La acción gravitatoria representa la componente tangencial del peso y puede aumentar o disminuir la resistencia al avance según se trate de una rampa o pendiente.

Como el valor de las pendientes es del orden de milésimas, se utiliza la expresión simplificada:

$$
R_{a g}=m * g * p
$$

Ecuación 4. Resistencia en rampa o pendiente

La masa se expresa en toneladas y la pendiente en milésimas.

\subsubsection{Fuerzas de tracción y de frenado}

La fuerza de tracción viene dada por la curva de tracción que se haya definido para el tren en cuestión. De igual manera, la fuerza de frenado, viene dada por las curvas de frenado eléctrico y frenado neumático.

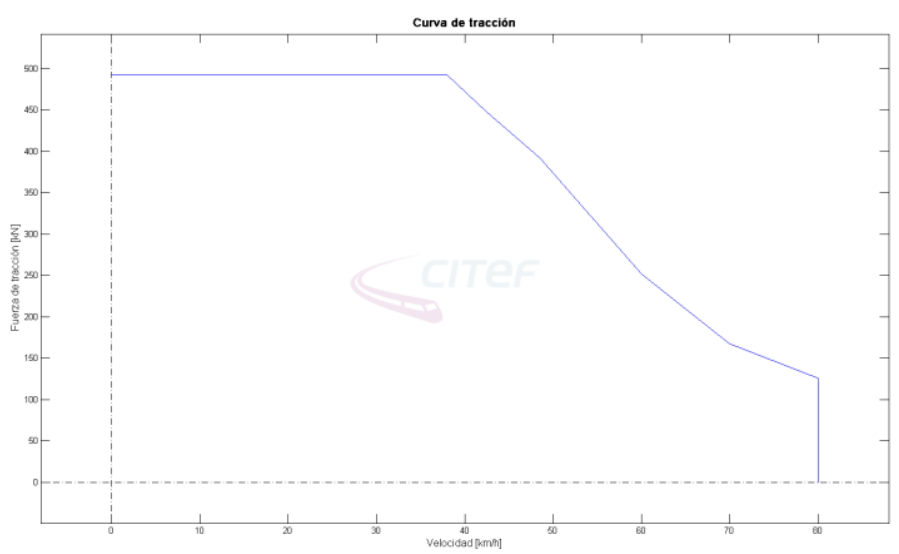

Figura 23 Ejemplo curva de tracción calculada 


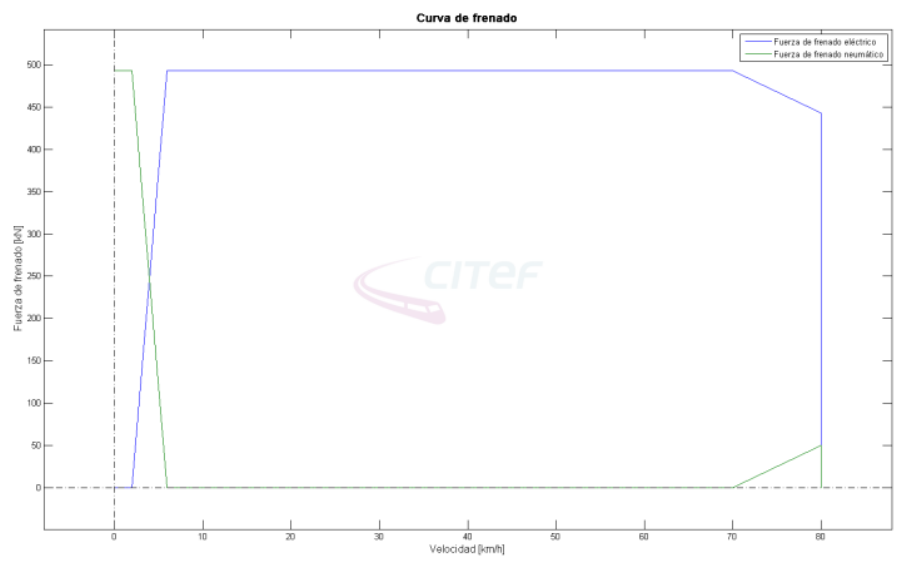

Figura 24 Ejemplo curva de frenado calculada

\subsubsection{Ecuación del movimiento}

La suma de todas estas componentes da la ecuación de movimiento del tren, bajo la forma de la Segunda Ley de Newton:

$$
\begin{array}{r}
F_{t r}-\left(A+B v+C v^{2}\right)-m \frac{K_{c}}{r}-m * g * p=m * g * a \\
\text { Ecuación 5. Balance de fuerzas aplicadas a la 2a ley deNewton }
\end{array}
$$

donde $\mathrm{m}$ es la masa total del tren $(\mathrm{M}+\mathrm{m})$ medida en $\mathrm{t}$

$\mathrm{g}$ es la fuerza de la gravedad medida en $\mathrm{m} / \mathrm{s}^{2}$

a es la aceleración medida en $\mathrm{m} / \mathrm{s}^{2}$

Resolviendo esta ecuación se obtienen los valores de la aceleración, para posteriormente resolver la integral, y de esta manera conocer la velocidad alcanzada al final del paso de simulación y el espacio avanzado. La masa $(\mathrm{m})$, trasladándolo al tren, se suele desglosar en la suma de la masa propia del tren más la masa (ruedas, ejes, discos de freno) [12].

\subsection{Cálculos eléctricos}

\subsubsection{Modelo de la sección transversal}

La alimentación de los sistemas de tracción ferroviaria se puede hacer a través de varios grupos de conductores, existiendo inducción magnética entre ellos en el caso de que la alimentación eléctrica sea mediante corriente alterna. El efecto del acoplamiento capacitivo se desprecia en este tipo de líneas puesto que es sólo importante en el caso de líneas muy largas y distancias muy cortas entre los cables.

El estudio del acoplamiento inductivo se refiere a la impedancia propia de un conductor con retorno por tierra y la impedancia mutua entre dos conductores aislados que tienen ambos retorno por tierra. Estas impedancias se determinan utilizando las 
expresiones desarrolladas por Carson, basadas en series que dependen de la disposición de los conductores.

Para el modelo se han considerado:

- La línea inductora es un conductor recto horizontal de longitud infinita en ambos sentidos.

- Las líneas entre las cuales ha de calcularse la impedancia mutua son paralelas entre sí.

- La tierra es homogénea, de resistividad finita con permeabilidad magnética relativa unitaria.

En el caso de que el sistema de alimentación sea mediante corriente continua, el modelo se simplifica al considerarse sólo el efecto resistivo en los conductores.

\subsubsection{Calculo de la matriz de impedancias de los conductores}

El modelo contempla la presencia por separado de las catenarias. Por tanto el modelo matemático contempla cinco hilos:

- Catenaria de la vía 1 (hilo de contacto, sustentador y feeder de refuerzo, si lo hubiera).

- Catenaria de la vía 2 (hilo de contacto, sustentador y feeder de refuerzo, si lo hubiera).

- Retorno (carriles, hilo de retorno).

- Feeder negativo de la vía 1.

- Feeder negativo de la vía 2.

Se construye una matriz [Z] que establezca una relación entre las caídas de tensión en los conductores $[\Delta \mathrm{V}]$ y las intensidades $[\mathrm{I}]$ de los mismos, de forma que

$$
\begin{aligned}
& \qquad[A V]=[Z] *[I] \\
& \text { Ecuación 6. Ecuación para conocer la caída de tensión en un circuito }
\end{aligned}
$$

Para simplificar el sistema que componen todos los conductores que forman parte de la electrificación de las líneas de tracción, se reduce el mismo a un número de grupos de conductores. Estos grupos están formados, cada uno de ellos, por conductores conectados en paralelo, es decir, con el mismo potencial.

El modelo abarca un mayor número de conductores, es decir los 14 conductores existentes en alimentación 2 X 25 con alimentador de refuerzo. 


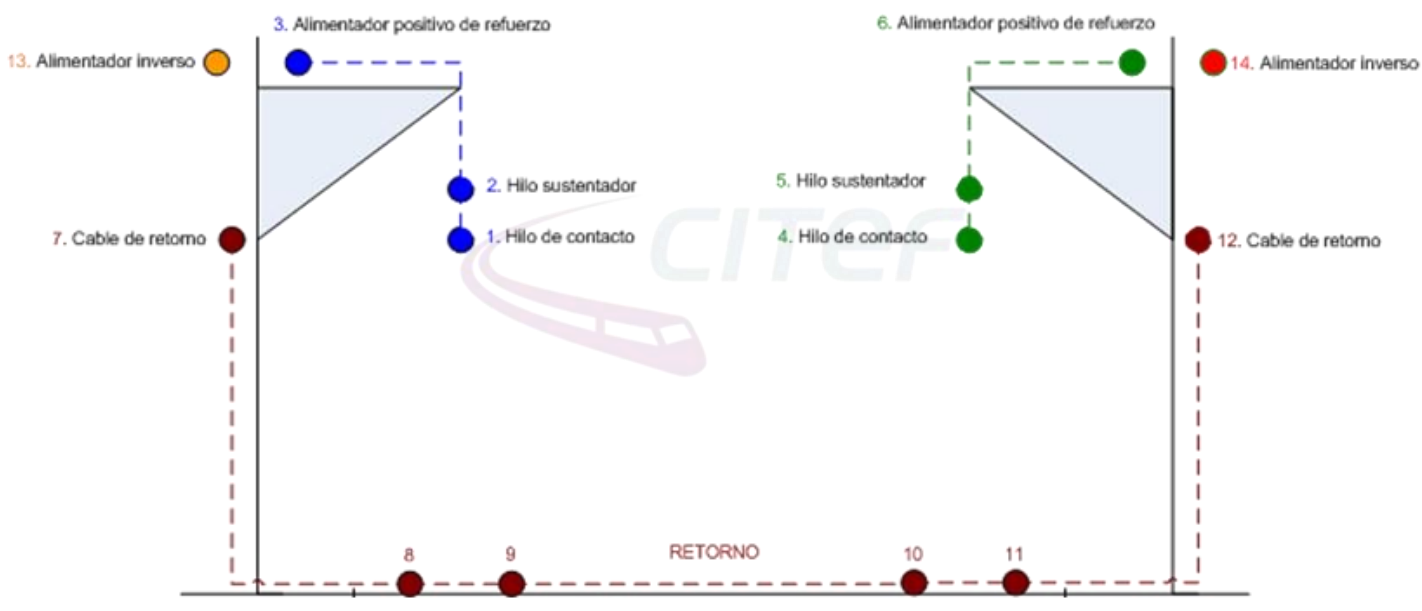

Figura 25 Sección transversal con los 5 tipos de conductores

Se define una matriz de impedancias que relacione magnitudes de estos cinco grupos. Se parte de las ecuaciones que definen el sistema completo (con los 14 conductores):

$$
\left[\begin{array}{c}
\Delta V_{1} \\
\vdots \\
\Delta V_{14}
\end{array}\right]=\left[\begin{array}{ccc}
Z_{1,1} & \ldots & Z_{1,14} \\
\vdots & \ddots & \vdots \\
Z_{14,1} & \ldots & Z_{14,14}
\end{array}\right]\left[\begin{array}{c}
i_{1} \\
\vdots \\
i_{14}
\end{array}\right]
$$

Ecuación 7. Sistema de matrices para cálculo de matriz de impedancia

La matriz de impedancias de $14 \times 14$ se reduce hasta convertirse en una matriz $5 \times 5$, que es la matriz de impedancias de la línea que se usará en los siguientes cálculos. Este tipo de técnica de simplificación ha sido estudiada y aplicada en diversos simuladores [13].

\subsubsection{Cálculo de la matriz de impedancias paralelo de los conductores}

La matriz de capacidades paralelas $[\mathrm{C}]$ no se puede obtener directamente a través de los parámetros de los conductores, sino que debe calcularse primero la matriz de coeficientes de potencial de Maxwell [P].

La matriz de coeficientes de potencial $[\mathrm{P}]$ es la que relaciona los voltajes de los conductores de la línea [U] con la cargas por unidad de longitud del conductor [q]:

$$
[U]=[P] *[q]
$$

\section{Ecuación 8.Cálculo de las tensiones en los conductores de la línea}

La matriz de coeficientes de potencial es real y simétrica, y sus elementos se calculan a partir de la geometría de los conductores. Una vez se tiene la matriz de coeficientes de potencial, para calcular la matriz de capacidades paralelas sólo hay que invertir la matriz:

$$
[C]=[P]^{-1}
$$


La unidad de las capacidades será F/ $/ \mathrm{km}$. Para la realización de la matriz de admitancias paralelo se consideran la matriz de capacidades y una matriz de conductancias [G]. Esta matriz de conductancias, que al tener en cuenta sólo las conductancias propias de los carriles, será una matriz con todos los elementos nulos excepto cuatro (los carriles).

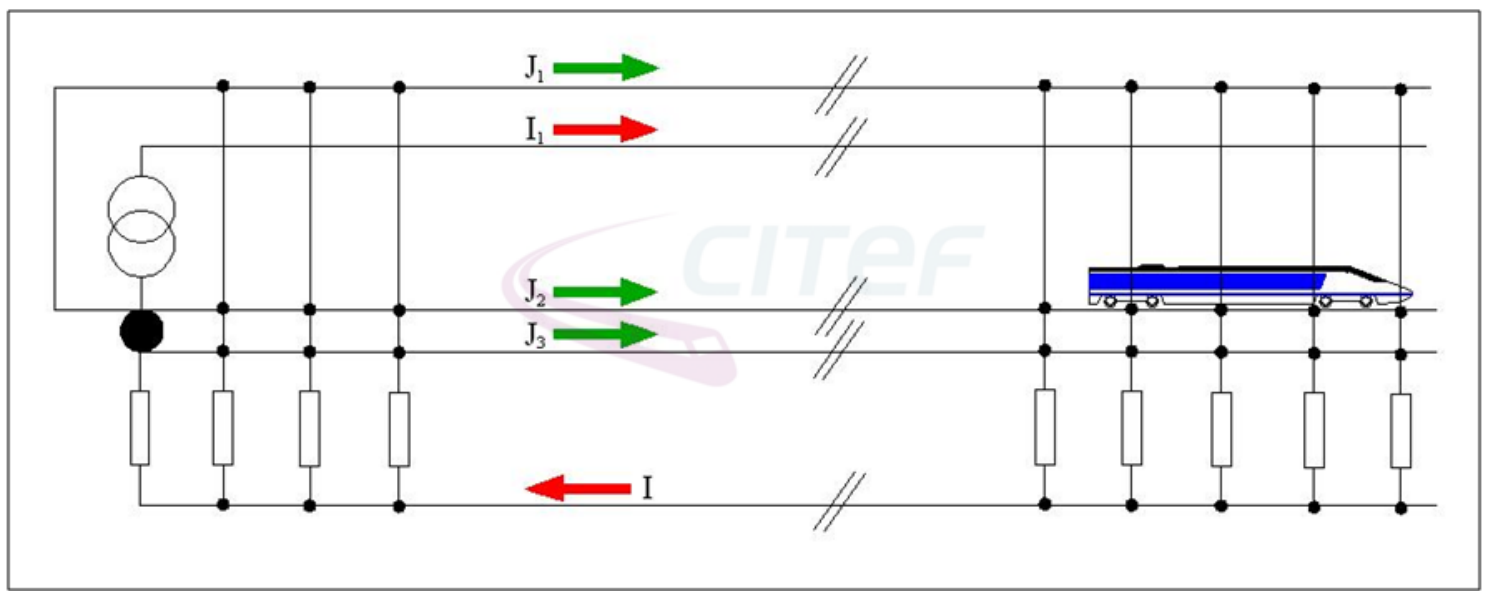

Figura 26 Cálculo de matriz de admitancias. Figura ejemplo

La matriz de admitancias paralelo es la suma de la matriz de capacidades y la matriz de conductancias:

$$
\left[Y_{p}\right]=[G]+j w *[C]
$$

Ecuación 10. Cálculo de la matriz de Admitancias del circuito a partir de matriz de Capacidades y de Conductancias

Al igual que con la matriz de impedancias, la matriz de admitancias paralelo de $14 \times 14$ se reduce hasta una matriz de tamaño $5 \times 5$.

\subsubsection{Cálculo de las tensiones, intensidades y potencia en la línea}

Para resolver el esquema eléctrico se emplea un análisis por nudos, por lo que los distintos elementos eléctricos que forman parte del sistema de alimentación (subestaciones, autotransformadores, trenes, cargas estáticas, etc.) se modelan mediante matrices de admitancias.

En la literatura y aplicación en los distintos simuladores, se han aplicado diversas técnicas de resolución. El método Newton-Raphson [14], tiene una aplicabilidad muy extendida a problemas no-lineales, y debido a las características propias del problema que se está tratando, también suele aparecer en la resolución de mallas eléctricas ferroviarias, aplicadas a la simulación. Para sistemas ferroviarios de alta velocidad y por tanto trabajando en corriente alterna, están los trabajos de [15] [16]

El objetivo es crear una matriz de admitancias global que agrupe todo el sistema, contemplando todos los ramales. 
Posteriormente, se calculan las tensiones e intensidades reales del sistema utilizando un proceso iterativo. Las intensidades que circulan por los elementos resistivos se definen mediante la potencia y el factor de potencia que están demandando. En primer lugar, se parte de tensiones supuestas en todos los nodos que conforman la red, y con dichas tensiones, potencias y factores de potencia se calculan los valores de las fuentes de intensidad. Una vez conocidas las corrientes, se resuelve el sistema para obtener los nuevos valores de tensiones en los nodos y se comparan con los valore supuestos. Si la diferencia entre ambos valores es mayor que una tolerancia definida, se repite el cálculo, utilizando como tensiones supuestas las obtenidas en el cálculo anterior.

De esta forma el sistema proporciona la solución del sistema eléctrico para un instante de tiempo determinado de movimiento de los trenes. Repitiendo el proceso desde el instante que sale el primer tren hasta el que llega el último tren a su destino se obtiene los valores eléctricos de la línea eléctrica para toda la simulación que posteriormente se puede estudiar.

\section{Convergencia}

Puede suceder, que la potencia demandada por los trenes, más la potencia de pérdidas en la línea, sean mayores que la potencia que físicamente puede suministrar la subestación. En este caso, no puede producirse convergencia porque el sistema físicamente no tiene solución.

Si estudiamos cómo es la evolución de la tensión en uno de los trenes en función de la potencia que está demandando, observamos que corresponde a una parábola. Para demostrarlo, estudiamos un circuito constituido por un generador real de tensión, una línea de impedancia dada, y una carga que está demandando una potencia $\mathrm{P}+\mathrm{jQ}$. Se ha sumado la impedancia de la línea con la del generador real, dando lugar a una impedancia $\mathrm{Zgl}=\mathrm{Zg}+\mathrm{Z}$

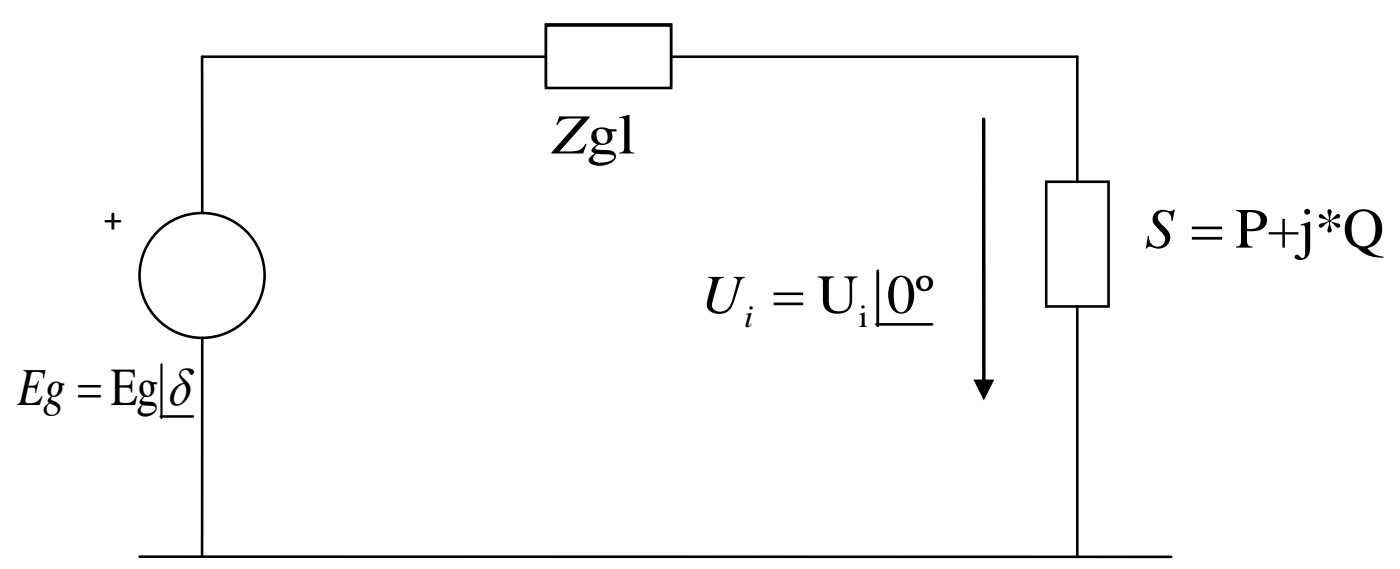




$$
\begin{aligned}
& E g=Z g l \cdot I+U_{i} ; \\
& E g\left\lfloor\delta=(\mathrm{R}+\mathrm{j} \cdot \mathrm{X}) \cdot \frac{\mathrm{P}-\mathrm{j} \cdot \mathrm{Q}}{\mathrm{U}_{\mathrm{i}}}+\mathrm{U}_{\mathrm{i}} ;\right. \\
& E g\left\lfloor\delta=\frac{\mathrm{R} \cdot \mathrm{P}+\mathrm{X} \cdot \mathrm{Q}+\mathrm{j} \cdot(\mathrm{X} \cdot \mathrm{P}-\mathrm{R} \cdot \mathrm{Q})}{\mathrm{U}_{\mathrm{i}}}+\mathrm{U}_{\mathrm{i}} ;\right. \\
& \mathrm{Eg}^{2}=\left(\frac{\mathrm{R} \cdot \mathrm{P}+\mathrm{X} \cdot \mathrm{Q}}{\mathrm{U}_{\mathrm{i}}}+\mathrm{U}_{\mathrm{i}}\right)^{2}+\left(\frac{\mathrm{X} \cdot \mathrm{P}-\mathrm{R} \cdot \mathrm{Q}}{\mathrm{U}_{\mathrm{i}}}\right)^{2} ; \\
& \mathrm{Eg}^{2} \cdot \mathrm{U}_{\mathrm{i}}{ }^{2}=\left(\mathrm{R} \cdot \mathrm{P}+\mathrm{X} \cdot \mathrm{Q}+\mathrm{U}_{\mathrm{i}}^{2}\right)^{2}+(\mathrm{X} \cdot \mathrm{P}-\mathrm{R} \cdot \mathrm{Q})^{2} ;
\end{aligned}
$$

Ecuación 11. Sistema de resolución del algoritmo eléctrico. Convergencia.

Por tratarse de un sistema no lineal, para cada potencia demandada, se tienen dos posibles soluciones, pero sólo la rama superior corresponde a una solución estable. A medida que hay más demanda de potencia, la tensión que ve el tren va siendo cada vez menor. Esta situación se mantiene, en el ejemplo de la gráfica inferior, hasta $9 \mathrm{MW}$, situación en la que no hay intersección con la curva Potencia-Tensión, con lo que no hay solución física posible para el sistema.

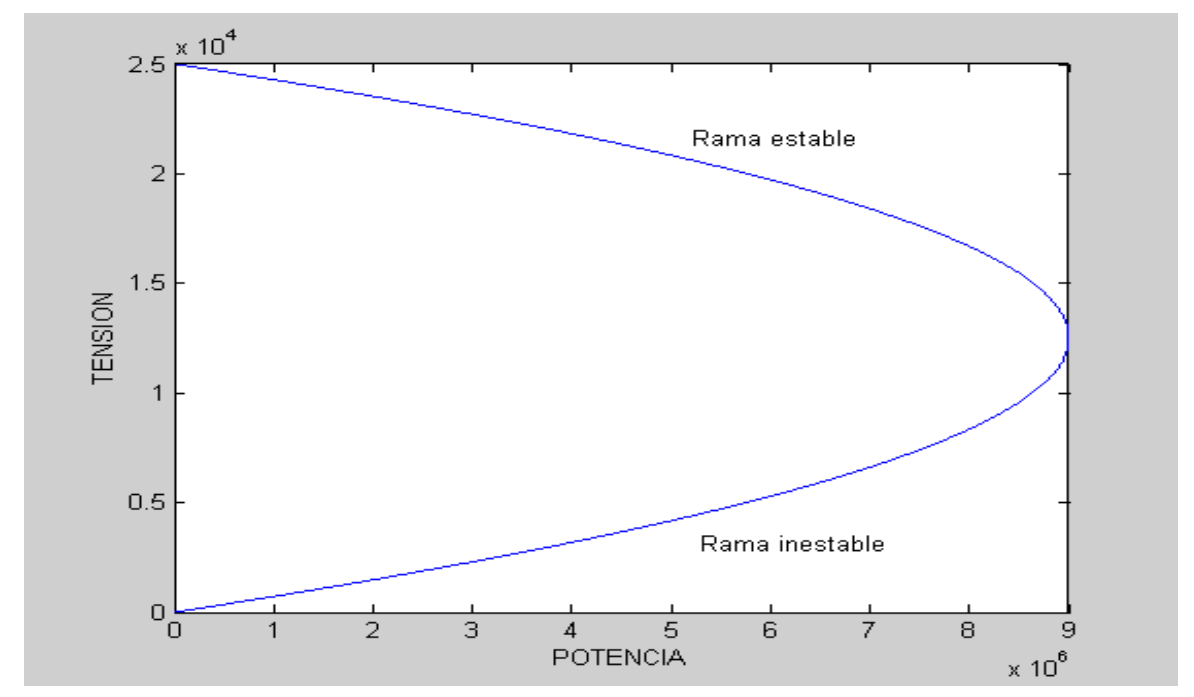

Figura 28 Gráfica explicativa de rama estable-inestable del criterio de Convergencia

Si se produce esta situación de no convergencia, la aplicación irá rebajando la potencia demandada por los trenes en un porcentaje parametrizable, hasta que el sistema converja.

Como salida de la de función Iterar tenemos dos variables que nos indican:

$$
\begin{gathered}
\text { control }=\left\{\begin{array}{c}
1 \rightarrow \text { hay reducción } \\
0 \rightarrow \text { no hay reducción }
\end{array}\right\} \\
\text { convergencia }=\left\{\begin{array}{c}
1 \rightarrow \text { no hay convergencia } \\
0 \rightarrow \text { hay convergencia }
\end{array}\right\}
\end{gathered}
$$


Al finalizar el proceso iterativo se obtienen las tensiones en cada nodo

\subsubsection{Cálculo de las intensidades de cortocircuito}

Para el cálculo de las intensidades de cortocircuito se usa el mismo modelo usado para el cálculo de la red de impedancias de la línea explicado previamente. En lugar de usar una carga representativa de un tren se posiciona una impedancia reducida entre la catenaria y el carril o entre el feeder negativo y el carril, obteniéndose las corrientes y tensiones a lo largo de la línea.

\subsubsection{Comprobaciones}

Durante el funcionamiento del sistema eléctrico ferroviario deben cumplirse tres condiciones de diseño, que corresponden a criterios de dimensionamiento eléctrico:

- La tensión en la catenaria debe estar comprendida entre los límites de diseño en cualquier punto del tramo.

- La corriente que circule por cada conductor no debe superar el valor máximo admisible para la elección de los conductores elegidos.

- La potencia demandada por los trenes no debe ser superior a la potencia nominal de los grupos de las subestaciones y de los autotransformadores.

- Se analizan también las tensiones de retorno entre carril-suelo. Importantes también para evitar corrosión del material conductivo y peligro potencial para personas y sistemas de telecomunicaciones.

Todas estas condiciones que caracterizan el correcto funcionamiento deben cumplirse tanto en situación de funcionamiento normal como en situaciones degradadas, y la aplicación se encarga de comprobarlo, indicando los casos en los que no es así y proponiendo soluciones para corregirlos.

\subsection{Resultados}

Una vez finalizada la simulación, se tienen una serie de resultados mecánicos y eléctricos que reflejan cuál ha sido el comportamiento del sistema.

Para conseguir estos resultados, tanto los eléctricos como los mecánicos, se han desarrollado funciones usando la versión de Matlab 13a. Recogen los resultados provenientes de las simulaciones, que se han ido almacenando en ficheros de texto, $y$ conociendo el formato de estos, puede calcular los valores pedidos y mostrar gráficas resultantes.

Entre los resultados mecánicos que se obtienen están la malla de circulaciones, que refleja el avance de los trenes respecto al tiempo, o las gráficas de velocidad y potencia demandada por los trenes frente al avance. Otro gráfico interesante que se muestra en la 
ocupación de los cantones por parte de los trenes, que es muy útil para saber la influencia de las circulaciones en los cálculos de las curvas de movimiento de los trenes y ver si existen conflictos no resueltos.

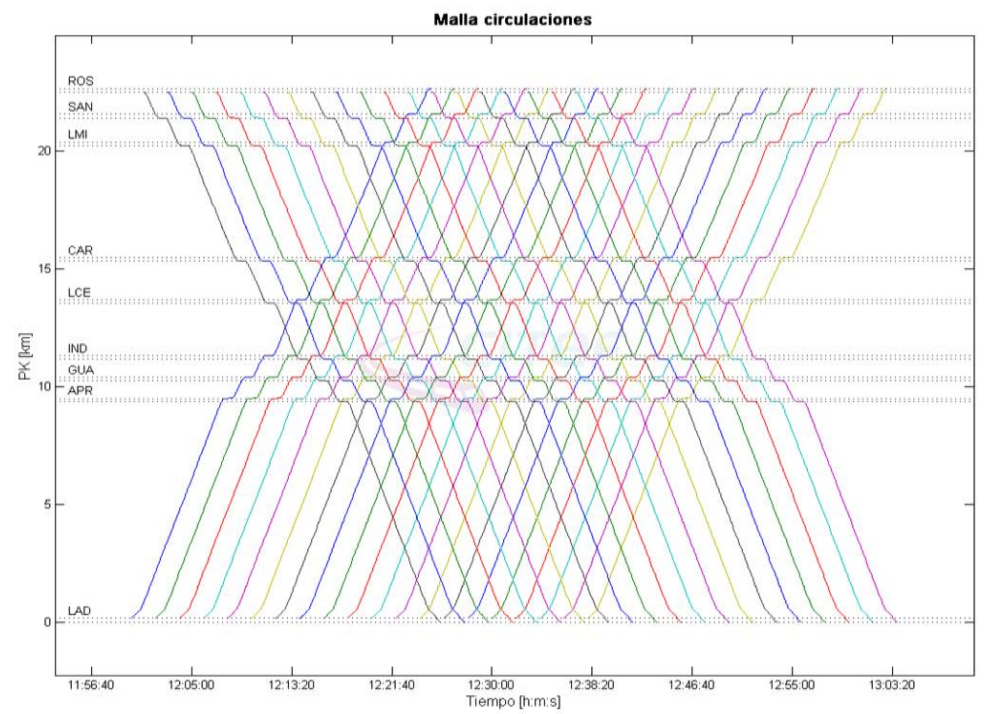

Figura 29 Ejemplo malla de circulaciones

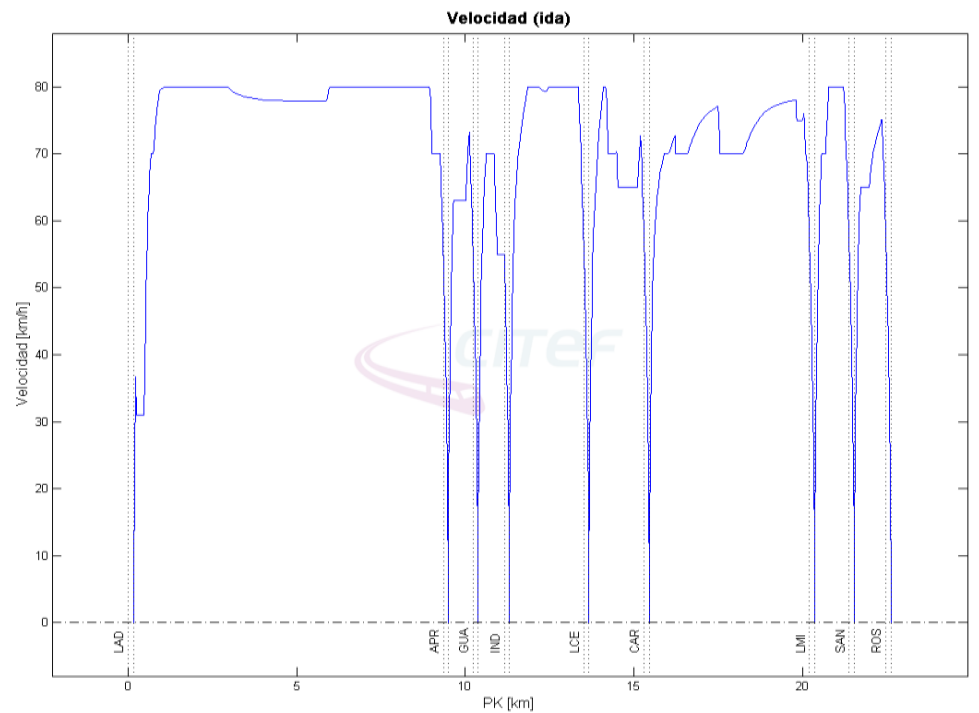

Figura 30 Ejemplo diagrama velocidad respecto del espacio 


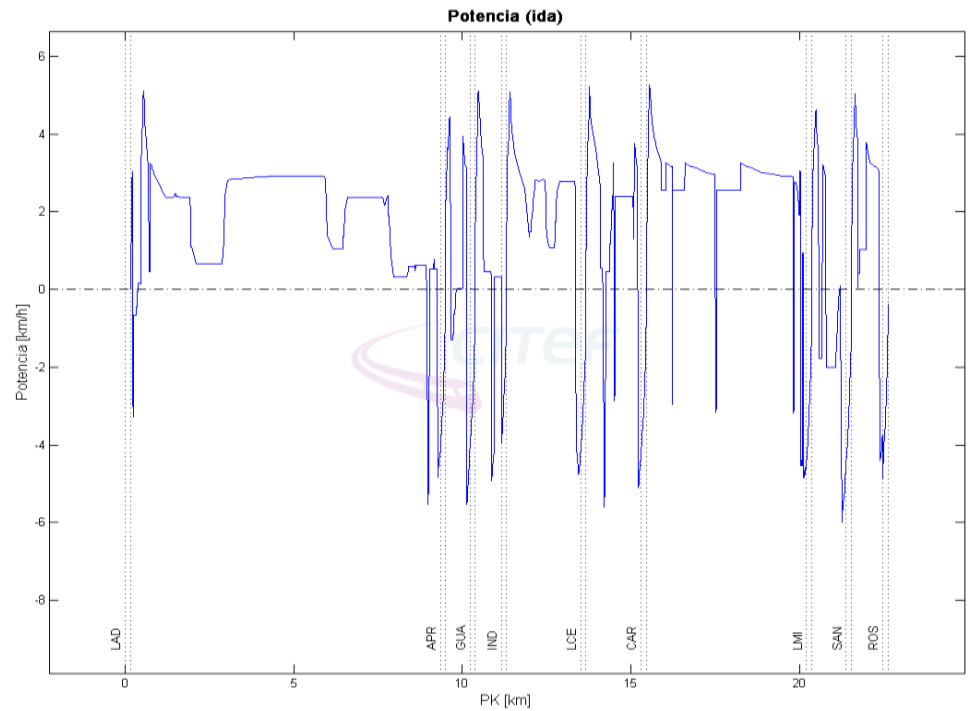

Figura 31 Ejemplo gráfica de potencia eléctrica demandada por un tren

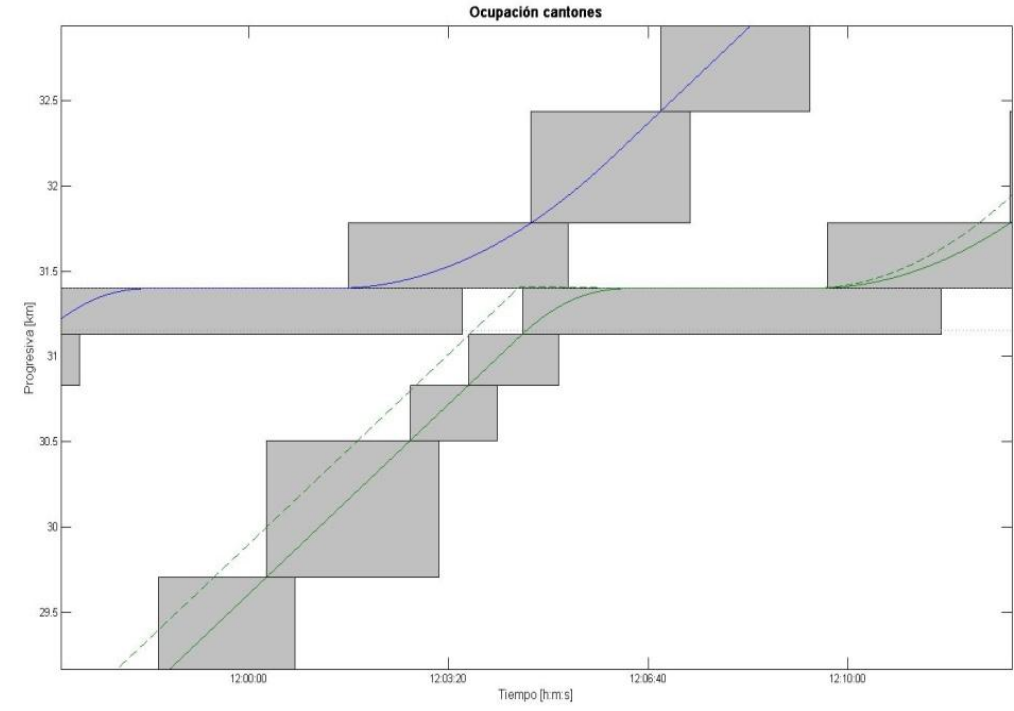

Figura 32 Ejemplo gráfica de ocupación de cantones

Respecto a los resultados eléctricos que se obtienen, se tienen la evolución temporal de la potencia suministrada por cada subestación o autotransformador, tensiones mínimas e intensidades máximas para todo el intervalo temporal en cada conductor, intensidades medias cuadráticas, etc. 


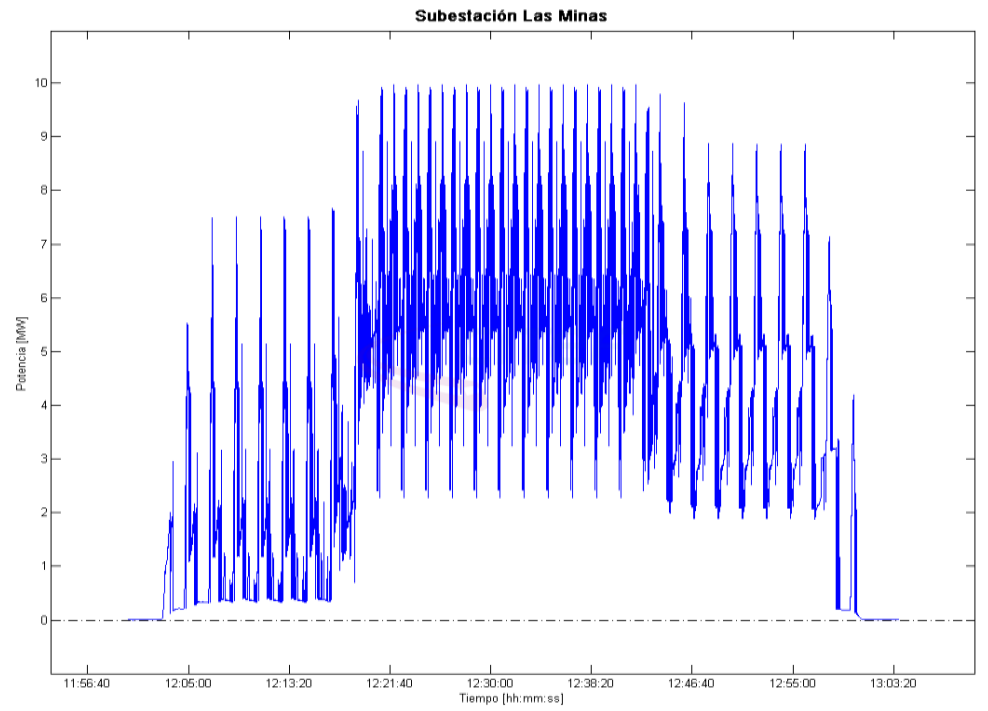

Figura 33 Ejemplo potencia aportada por una subestación de tracción

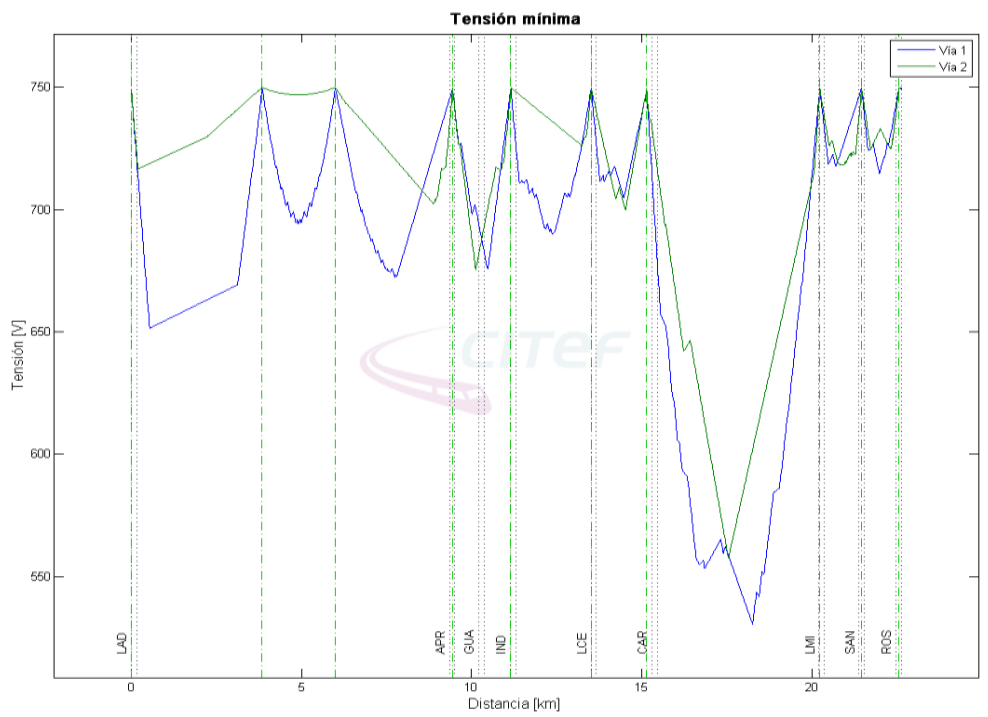

Figura 34 Ejemplo de gráfica de análisis de tensiones mínimas en pantógrafo 


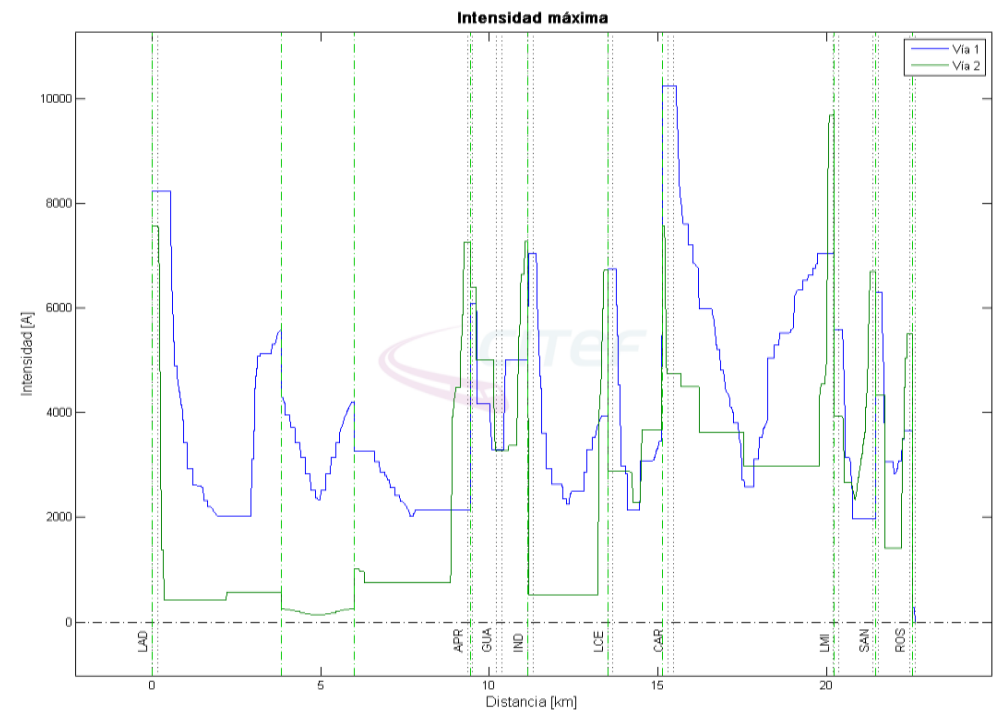

Figura 35 Ejemplo gráfica intensidad máxima en conductores

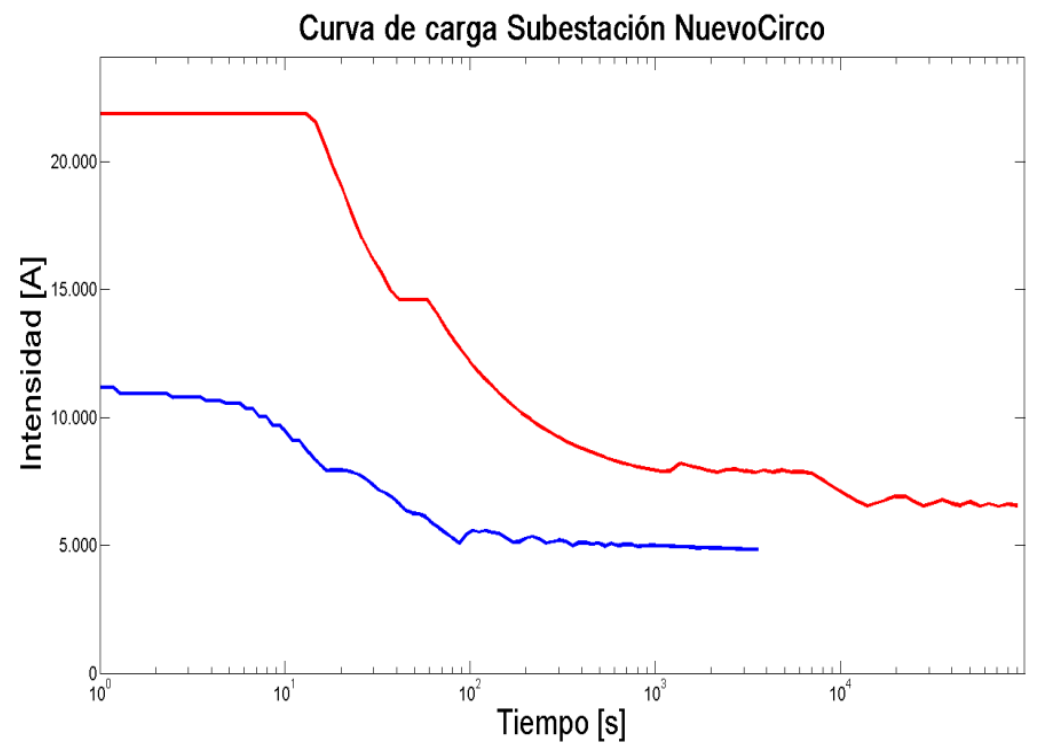

Figura 36 Ejemplo curva de sobrecarga de una subestación de tracción 


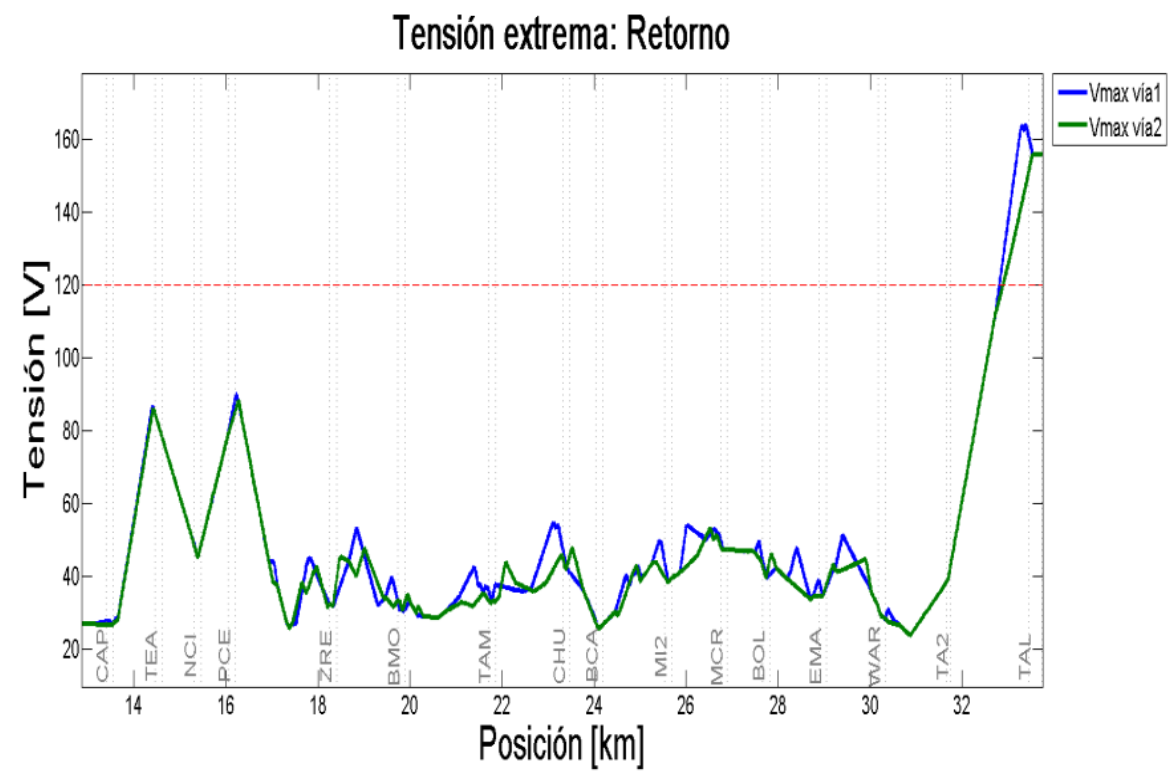

Figura 37 Ejemplo estudio de tensiones de retorno carril-tierra

En estudios más recientes se han analizado los anillos de distribución de la red de media tensión hacia el sistema eléctrico de la red ferroviaria. La idea es muy similar a la que se aplica para la red de tracción, pero trasladada a la red de distribución.

El simulador ha sido probado en proyectos que han exigido una continua adaptación a las exigencias que marcaban las características del sistema ferroviario a estudiar. La evolución evidentemente no es completa, y se sigue mejorando el sistema e integrando nuevas funcionalidades al mismo. En los niveles de señalización y a nivel eléctrico es donde se plantean los desafíos más notables. Estudio de desequilibrios en la red, poder integrar sistemas de regeneración de energía o subestaciones reversibles o estudio de las corrientes inducidas, son algunos de los problemas a los que deberemos ir dando respuesta en futuros desarrollos. 


\section{bibliografía}

[1] A. H. Velilla, E. P. de la Fuente y L. R. Rodríguez, «SILVIA: una herramienta de diseño de la electrificación de ferrocarriles de alta velocidad,» Anales de mecánica y electricidad, vol. 79, no 6, pp. 8-16, 2002.

[2] S. Ratés-Palau, Modelización de los sistemas de electrificación ferroviaria, en corriente alterna y continua, con sistemas recuperadores de energía para el estudio de la eficiencia energética, 2012.

[3] M. T. Söylemez y S. Açıkbaş, «Energy loss comparison between 750 VDC and 1500 VDC power supply systems using rail power simulation," de Int. Conf. on Comp. Aided Design, Manufacture, and Operation in the Railway and Other Advanced Transit Systems (COMPRAIL 2004), Dresden, Germany, 2004.

[4] A. Nash y D. Huerlimann, «Railroad simulation using OpenTrack,» de Computers in railways IX, 2004.

[5] L. Abrahamsson, Railway Power Supply Models and Methods for Long-term Investment Analysis. Licenciate Thesis, Estocolmo: Royal Institute of Technology (KTH), 2008.

[6] J. Demitz, C. Hübschen y C. Albrecht, «Timetable stability using simulation to ensure quality in a regular interval timetable," de TimeTable Planning and Information Quality, WITT PRESS, 2010.

[7] E. Roanes-Lozano, E. Roanes-Macías y L. M. Laita, «Railway interlocking systems and Gröbner bases," Mathematics and Computers in Simulation, vol. 51, no 5, pp. 473-481, 2000.

[8] C. Schiffers y G. Hans, «IEEE standard for communications-based train control (CBTC) performance and functional requirements, " de Vehicular Technology Conference Proceedings, 2000.

[9] R. Bloomfield, «Fundamentals of European Rail Traffic Management System - ERTMS,» de 11th IET Professional Development Course on Railway Signalling and Control Systems, 2006.

[10] C. Vera, «Prestaciones de los vehículos ferroviarios, " de Ferrocarriles, Madrid, Universidad Politécnica de Madrid, 2003, pp. 1-44.

[11] W. J. Davis, «The tractive resistance of electric locomotives and cars,» General Electric, 1926.

[12] A. García-Álvarez, «Dinámica de los trenes en Alta Velocidad,» Fundación de los Ferrocarriles Españoles, 2005. 
[13] H. W. Dommel, "Computation of Cable Impedances Based on Subdivision of Conductors," IEEE Transaction on Power Delivery, vol. 2, no 1, pp. 21-27, 1987.

[14] T. J. Ypma, «Historical development of the Newton-Raphson method," SIAM, vol. 37, no 4, pp. 531-551, 1995.

[15] P. H. Hsi y S. H. Chen, «Electric load estimation techniques for high-speed railway (HSR) traction power systems, "IEEE Transactions on Vehicular Technology, vol. 50, no 5, pp. 1260-1266, 2001.

[16] E. Pilo, L. Rouco y A. Fernández, "A simulation tool for the design of the electrical supplysystem of high-speed railway lines," de IEEE PES Summer Meeting, Denver, 2000.

[17] J. Stern, «TrackFeed Simulation Reference Manual,» Tech. Rep. BBSE951112-BNA, Balfour Beatty Rail, 2006. 


\section{Metodología general}

Este capítulo se ha fraccionado en cuatro apartados. En primer lugar se introducen los fundamentos de los algoritmos de optimización escogidos para el sistema general. En segundo lugar se describen los elementos que van a ser importantes en el análisis de la optimización, y cómo se van a valorar estos elementos. El tercer apartado está dirigido a explicar la problemática de encontrar un parámetro o situación válida, de forma que sea capaz de aportar información acerca de las necesidades de la explotación en términos de energía. La conclusión final de este subapartado deriva en el baremo de medida que se va a usar en los procesos de optimización. Finalmente en el último subapartado se describirá la función objetivo general a aplicar, así como las restricciones que deberá cumplir el modelo.

\subsection{Algoritmos de optimización aplicados a problemas de Ingeniería}

Como proyecto de ingeniería que se cataloga este trabajo, y teniendo el objetivo de optimizar una parte del diseño del dimensionamiento eléctrico, una de las tareas principales ha sido encontrar y adaptar un método de optimización válido para este problema.

Los progresos y estudios de los distintos métodos de optimización aplicados a la ingeniería, han ido creciendo en adaptación a los problemas, conforme se estudiaban las particulares características de cada uno de ellos. Es evidente también que el aumento en la potencia y capacidad de los equipos de computación, ha sido un acicate para que estos métodos se fueran cada vez más, implantando en proyectos de ingeniería. No solo eran necesarios los métodos de optimización clásicos, cuyos pioneros fueron Lagrange, Fermat, Euler o Newton, sino que fue necesaria la recreación de aspectos sacados de la naturaleza, para combinar con los problemas ingenieriles, todo ello apoyado por la potencia computacional.

Como paso previo a la descripción de los algoritmos de optimización elegidos, se describen los distintos métodos que se han encontrado en la literatura. Esta lista pretende detallar qué ramas existen en la actualidad y cuáles son los parámetros por los que se rigen cada una de ellas, y por qué son adecuadas para determinados problemas. Para esta diferenciación, existen diversos trabajos que destacan por la rigurosidad, por su explicación detallada y concisa [1] [2], como por las referencias que aportan.

En [1], se presentan y clasifican los problemas de optimización y métodos para resolverlos. Los autores parten de que para llegar a saber de qué tipo de problema de optimización se está hablando, y posteriormente cómo solucionarlo, es imperativo conocer los siguientes aspectos:

- Problemas lineales, no lineales o mixtos.

- Si las variables del sistema son continuas o discretas, o mixtas. 
- Si el problema es convexo o no convexo. Es decir, si el problema tiene como resultado una solución única global o, puede satisfacerse con soluciones óptimas locales.

- Si las funciones representativas del problema son o no diferenciables.

Por tanto, lo primero que se debe examinar cuando se desea optimizar son los puntos anteriormente descritos. A partir de conocer esto, se pasaría a conectar el problema que se intenta optimizar con la rama teórica de optimización adecuada.

\section{- Programación lineal}

El modelo que está basado en este tipo de problemas es aquel en que sus ecuaciones, tanto las funciones objetivo como las representativas del modelo de restricciones que trata de optimizar, son lineales. El método simplex [3], es el que por convenio se suele utilizar para las optimizaciones de este tipo de problemas.

El tipo de variables también determina el tipo de problema, y en este caso, solo es aplicable cuando son todas variables continuas.

\section{- Programación no lineal}

Esta técnica se debe aplicar a problemas en las que alguna función objetivo o restricciones asociadas al problema, no son lineales. El objetivo final es maximizar/minimizar la función objetivo. Los autores en [1] detallan que se puede determinar una subclase dentro de la programación no lineal, que sería la programación cuadrática (QP). En relación a esta subclase, uno de los algoritmos más extendidos en problemas de ingeniería es el SQP (Sequential Quadratic Programming) [4]. En diversos trabajos se ha usado esta técnica, tales como en trabajo de consumo eficiente de energía basado en supercondensadores a bordo del tren [5]. Otro trabajo interesante se puede encontrar en [6], ya que los autores utilizan esta técnica para agilizar la búsqueda de óptimos locales y de esta manera complementar las deficiencias en este aspecto que tiene el otro algoritmo usado HSA (Harmony Search Algorithm [7]).

SQP aplica el equivalente del método de Newton a las condiciones de Karush Kuhn Tucker :

$$
\begin{gathered}
\nabla f\left(x^{*}\right)+A\left(x^{*}\right) \lambda+C\left(x^{*}\right) v=0 \\
h\left(x^{*}\right)=0 \\
g\left(x^{*}\right)+s=0 \\
S V e=0 \\
(s, v) \geq 0
\end{gathered}
$$


Donde $e=[1,1, \ldots, 1]^{T}, \lambda$ es el vector de multiplicadores de Lagrange de la restricciones de igualdad y $\mathrm{v}$ de las restricciones de desigualdad.

Al igual que para la Programación Lineal, en este caso solo aplica cuando todas las variables del modelo son continuas.

\section{- Programación lineal mixta}

Dentro de la clasificación que tenemos como referencia, teniendo en cuenta el tipo de variables que forman el problema, en este caso, como su nombre indica, estarían representados casos de variables continuas y discretas.

$$
\min Z=a^{T} x+b^{T}\left\{\begin{array}{c}
A x+B y \leq d \\
x \geq 0, y \in\{0,1\}^{n}
\end{array}\right.
$$

Ecuación 2. Sistema ecuaciones programación lineal mixta

Según los autores en [8], la resolución de este tipo de problemas pasaría por acotar los subproblemas lineales para aplicar el método simplex que se ha comentado en el apartado de Programación Lineal. Para ello, la estrategia a seguir es la de ramificación y poda. En el peor de los casos se obtendría un árbol completo, con todas las soluciones posibles. Las distintas evoluciones en trabajos con problemas de este tipo, se dirigen sobre todo a mitigar esta última posibilidad, acotando lo mejor y más rápidamente posible la cantidad de soluciones a evaluar. Otros enfoques para evitar que se evalúen todas las posibilidades, las estudiaron en [9] y [10].

\section{- Programación no lineal mixta}

Los también llamados en su acrónimo en inglés MINLP, contienen en su conjunto aquellos problemas formados por variables discretas y continuas, y que además alguna de sus restricciones o función objetivo es no lineal. El sistema de ecuaciones de un MINLP suele tener las siguientes características:

$$
\begin{gathered}
\min Z=f(x, y)\left\{\begin{array}{c}
g_{j}(x, y) \leq 0 j \in J \\
x \in X, y \in Y
\end{array}\right. \\
\text { Ecuación 3. Minimización usado técnica programación no lineal mixta }
\end{gathered}
$$

y donde otra de las casuísticas para examinar el tipo de problema entra en juego, la convexidad. En esta definición las funciones $\mathrm{f} y \mathrm{~g}$ son convexas $\mathrm{y}$ diferenciables, y $x$ e $y$ son las variables discretas y continuas respectivas del método mixto. 
Las técnicas que suelen aplicarse para solventar este tipo de problemas son:

○ Ramificación y poda

Es una extensión del método ya explicado para problemas lineales de misma naturaleza. Evidentemente en este caso, los problemas a acotar serían no lineales. Solo se debería optar por este método cuando los subproblemas NLP no son complejos en su resolución o, en definitiva, tienen un peso pequeño en el conjunto de nodos del árbol.

\section{○ Aproximación exterior}

En el trabajo presentado por [11], el objetivo es encontrar una solución de un problema singular NLP, para a partir de esta resolución ir acotando el problema. De este modo estaríamos hablando de una técnica mixta, usando variantes de las anteriores, ya que no trabaja directamente con el problema maestro y además realiza cortes en la búsqueda en el espacio de soluciones. Este método tiene la característica de necesitar pocos ciclos de iteraciones en sus primeras aproximaciones.

\section{○ Descomposición Benders generalizada}

En el trabajo desarrollado en [12] se explica la técnica y también sus principales partes y tipología que la definen. Es una técnica que se asemeja bastante a la de Aproximación exterior. La diferencia radica en cómo se crea el Problema Maestro, que debe ser también problema mixto lineal. En este caso solo se tienen en cuenta las desigualdades activas y el conjunto que las contiene.

\section{- Plano de corte extendido}

La característica principal de este método [13], es que los subproblemas NLP no intervienen en el mismo. El camino que sigue este método para conseguir la optimización, es solo iterando con el problema Maestro MIP (M-MIP), de modo que las restricciones que más se cumplen, las convierte a lineales en un punto determinado. La convergencia la alcanza cuando se sobrepasa un límite preestablecido de violaciones de este tipo de restricciones.

Los problemas acotados por los MINLP se encuentran en muchos campos de la ingeniería y por tanto han sido muy estudiados. Esto se verifica en la cantidad de alternativas o extensiones de las ramas de resolución planteadas anteriormente, que se encuentran en funcionamiento o en caminos investigados. Algunas de ellas son:

- MIQP-mp (Mixed integer quadratic problems), como introduce Leyffer en sus trabajos [14]. Dentro de las técnicas aplicadas en la Aproximación Exterior, se encuentran este tipo de problemas. Esta variante se presenta cuando el problema 
no es lineal, tiene factores cuadráticos en su modelo de ecuaciones, y por tanto exige otro tipo de variantes para ser abordadas. Se pretende modelar el problema para que sea posible trabajar en una simplificación de MINLP.

- Reduciendo la dimensionalidad del Problema Maestro dentro de los problemas referidos a la técnica Aproximación Exterior. El gran número de restricciones que están inmersos en este tipo de problemas, hace que crezca la complejidad. Se trata de paliar este inconveniente, reduciendo precisamente el número de restricciones del MINLP.

- Gestionando mejor las igualdades $h(x, y)=0$. Cuando la ecuación es no-lineal, como primer obstáculo existe la dificultad de linealizarla, ya que es probable que en algunos puntos no sea posible. Un aspecto muy relevante es que esta no linealidad introduce ciertas pautas de no convexidad del problema, por lo que se suele relajar el propóstio de encontrar una solución óptima usando diversas técnicas, como presentan Kocis y Grossman [15].

- Gestionando las no-convexidades. Una de las vías de trabajo para evitar el efecto de no-convexidades en alguno de los subproblemas o Problema Maestro, es intentar reducir el efecto que producen. La propia naturaleza de este tipo de problemas proporciona detalles de que es muy probable que no se obtenga el mínimo local, por lo que en algunos casos se plantea incluso de aplicar técnicas heurísticas para conseguir el resultado mejor posible.

Todas las técnicas relatadas se tienen que ver desde un punto de vista computacional. El trabajo para solucionar los problemas debe contemplar la perspectiva de desarrollar un programa que se pueda ejecutar en una computadora. Este tipo de problemas tienen un amplio espectro de programas que los solventan. Ejemplos serían el GAMS [16] o MINOPT [17], este último muy dirigido a la resolución de problemas por medio de la Aproximación Exterior, que se ha comentado brevemente.

\section{- Optimización dinámica}

El modelo de los problemas representados por esta rama de trabajo, son los que por medio de ecuaciones diferenciales pueden determinar su comportamiento dinámico, y por tanto se pueden encontrar en cualquier rama de la Ingeniería. Los principales caminos para trabajar con la optimización dinámica, según Biegler y Grossman [1] serían los siguientes:

\section{- Métodos variacionales}

Método clásico de la programación de Control, basado en el método de Máximo de Pontriaguin [18], donde se busca modelar el sistema por medio de su Hamiltoniano. Este método se encuentra en muchos casos de resolución aplicados en la Ingeniería, y más concretamente se ha encontrado en muchos estudios de búsqueda de optimización de la energía aplicada a sistemas ferroviarios. 


\section{- Discretización parcial}

Quien opta por este sistema de optimización, tiene en cuenta dos fórmulas para atacar el problema, o bien usando Programación dinámica [19] aplicado en espacios de problemas relativamente pequeños y para intentar evitar óptimos locales; o bien Métodos secuenciales directos [20]. En general la forma de actuar es discretizando solo las variables de control para a continuación trabajar con un motor de resolución de ecuaciones diferenciales en cada iteración. El método de secuenciales directos se aplicaría solo en caso de que el sistema fuera estable.

\section{- Discretización total}

Combina la aplicación del método de resolución SQP (Sequential Quadratic Programming) a todos los subproblemas no lineales de programación y además, como su nombre indica, discretiza todas las variables que aparecen en el modelo. Para la discretización de las variables existen un par de aproximaciones, Disparo múltiple [21], se discretiza el tiempo en etapas, y los Métodos de Colocación [22], donde se trabaja con elementos finitos.

\section{- Optimización global}

A pesar de que los algoritmos anteriores tienen una gran aceptación como aproximación al problema de optimización en ingeniería, hay casos en los que no son la mejor opción. Estos casos se presentan cuando encontrar el óptimo global es ineludible. Las técnicas anteriores no siempre garantizan la convexidad del resultado final, por lo que es necesario optar por otro tipo de técnicas, que son las aplicadas en la optimización global. Las técnicas de optimización global deben construir su modelo teniendo en cuenta que van a tener que trabajar todo el espectro posible de soluciones del problema, y de esta manera diferenciarse claramente de los algoritmos que encuentran óptimos locales.

Los modelos de optimización global se dividen en:

- Deterministas

No tienen en cuenta ningún factor aleatorio en la búsqueda del óptimo. Los métodos más frecuentes en la aplicación de este campo son:

- Métodos de ramificación y bordes [23]

- Métodos de intervalo [24]

○ Métodos de puntos MultiStart [25]

○ Métodos Lipschitzian [26]

- Estocásticos

Estos métodos estarían definidos dentro del conjunto de problemas en los que existe fuertes condicionamientos que impiden el uso de técnicas deterministas, 
como pueden ser que tengan factores que impidan la certeza absoluta de alguna de sus variables o bien también se pueden definir como aquellos que no se tiene la posibilidad de trabajar con sus derivadas o vienen afectadas por fuertes imprecisiones en sus cálculos.

Como se ha ido explicando en anteriores apartados, la división entre problemas lineales y no lineales, es una etapa primordial durante la clasificación. Con las técnicas metaheurísticas, la resolución de ambas vertientes está bastante extendida en la literatura, no así con los problemas mixtos, que tienen una adaptación mucho más compleja que con los modelos puros, ya sean con variables continuas o discretas.

Las características básicas que definen estas técnicas son:

- El alcanzar el óptimo global no está garantizado.

- Es posible que durante la evolución de la búsqueda de la solución, se elija un camino que es peor que los anteriores encontrados.

- Existe la obligatoriedad de calcular o estimar un punto de parada a la búsqueda, ya que por sí solas no son capaces de reconocer el óptimo.

- Se pueden aplicar a todo tipo de problema que acepte las condiciones iniciales de este tipo de modelos, que no tengan derivadas.

- Entre otros operadores, uno de los más importantes es el de selección y en ningún caso obedece a una base de datos que se haya ido construyendo a lo largo del camino, si no que se tratará la evaluación de los individuos en el mismo ciclo y con la información que se tenga en ese momento.

La evolución de las técnicas basadas en la búsqueda de soluciones de forma aleatoria se está acelerando conforme se van aplicando las nuevas tecnologías. Las pioneras se situarían en la segunda mitad del siglo XX, con métodos de búsqueda directa. Uno de los principales sería el de Nelder-Mead [27], cuya característica básica sería que no tenían en cuenta las restricciones del problema.

Aunque las soluciones que ofrecen los técnicas metaheurísticas no son las óptimas y, en general, ni siquiera es posible conocer la proximidad de las soluciones al óptimo, permiten estudiar problemas de gran complejidad de una manera sencilla y obtener soluciones suficientemente buenas en tiempos razonables.

Aparte de la corriente basada en búsqueda directa, la gran mayoría de técnicas que se aplican, y por supuesto que se están desarrollando y estudiando más, son aquellas que buscan un comportamiento parecido en procesos de la naturaleza, más concretamente en teorías evolutivas, tal y como establece en [28]:

- Búsqueda Tabú [29]. Se basa en realizar búsquedas por criterios de vecindad donde se va guiando la búsqueda del óptimo con la ayuda de una Memoria. En esta Memoria asociada al algoritmo, se almacenan las soluciones ya visitadas, 
que en el momento de volver a tratar con ellas se descartan, y por tanto se dirige el algoritmo por otros caminos no visitados o no tabú.

- Técnicas asociadas a Computación Evolutiva

○ Algoritmos genéticos [30]

- Estrategias Evolutivas [31]

- Programación Evolutiva [31]

- Recocido simulado [32]

- Enjambre de hormigas [33]

Uno de los más importantes precursores de la aplicación de estas teorías a resolución de problemas complejos es Holland [34]. Es el primero que hizo referencia a cómo adaptar modelos de optimización matemática a teorías de evolución, concretamente a cómo los individuos con mejor genética suelen transmitir con mayor probabilidad esos mismos genes a individuos de generaciones siguientes. Dentro de las teorías evolutivas se enmarcan un par de corrientes que se nutren de la misma idea. Las Estrategias Evolutivas y la otra es la Programación Evolutiva, cuyo máximo exponente serían los trabajos iniciales de Back [31]. En definitiva las tres técnicas englobadas en la computación evolutiva, tienen diferencias a bajo nivel, pero lo que se observa en una capa superior es muy similar entre ambas:

- Estudio del modelo del problema y definir el genotipo que va a identificar a cada posibilidad de solución, además de la función objetivo para evaluar a cada uno de ellos.

- Generación inicial aleatoria de la primera población y evaluación posterior de cada uno de los individuos mediante la aplicación de la función objetivo

- Aplicación de las restricciones que impida que un individuo sea portador de una solución, ya que viole las condiciones de alguna de las restricciones.

- Finalmente se procesarán los datos obtenidos por cada individuo para dar paso a la creación de una nueva generación, normalmente usando los operadores clásicos de selección, cruce entre individuos para dar nuevos y mutación, alterando los ya existentes.

Repitiendo estos pasos se debería llegar al objetivo final, consiguiendo cada vez una generación mejor que la anterior. La gran adaptabilidad a problemas complejos y también la simplicidad con la que se pueden modelar las optimizaciones a realizar, han hecho que la popularidad de estas técnicas haya crecido a lo largo de su corta historia. No es óbice que el amparo de una cada vez más potente tecnología de la información, también ha aportado mucho para el auge de estas técnicas. Una gran cantidad de simuladores y de paquetes de optimización, han ido apareciendo para dar cobertura a los usuarios de las Estrategias Evolutivas. 


\section{- Algoritmos genéticos Multiobjetivo}

Conforme han ido apareciendo nuevas formas de trabajar usando los algoritmos genéticos o estrategias evolutivas, y conforme se han ido adaptando a nuevos problemas y más concretamente en la ingeniería, se ha necesitado de una nueva visión a la hora de atacar estos problemas. Este nuevo punto de vista debía tener en cuenta aquellos problemas en los que existían objetivos contrapuestos, es decir, lo óptimo de uno de ellos era muy probable que no fuera lo óptimo para el otro. El Frente de Vilfredo Pareto [35] se suele usar como paradigma de la resolución de este tipo de conflictos de intereses. En todo espacio de soluciones multiobjetivo, existe un Frente o Barrera en la que los individuos frontera tienen algún valor de los objetivos que es el mejor de todas las soluciones encontradas hasta el momento, es decir, son individuos no-dominados. Con esta Barrera es con la que se debe tomar la decisión final, y que le corresponderá al encargado dependiendo de la causa y variables independientes que se atengan a cada individuo, por tanto ya no interviene el algoritmo sino que es decisión final de la persona. Estos algoritmos poseen una ingente cantidad de investigadores que evolucionan por momentos la forma de afrontar el problema. Quizás entre los más destacados estarían los trabajos de Veldhuizen y Lamont [36] y también del profesor Coello [37].

Las mejoras que se van aportando en cada algoritmo tratan de subsanar versiones anteriores en función de la complejidad temporal total, la capacidad de convergencia hacia óptimos globales, la versatilidad respecto del modelo a interpretar por parte del algoritmo evolutivo, el enfoque hacia la importancia de las restricciones en el desarrollo de la evolución del algoritmo,... 


\subsection{Elección de la Técnica de Optimización. Criterios clave y motivación.}

El primer paso para adentrarnos y conocer las distintas posibilidades y caminos que se podían tomar para optimizar el proceso de esta Tesis, se ha descrito anteriormente. Se ha buscado, estudiado y clasificado según las capacidades, características y ventajas y desventajas de cada modelo de optimización, la que más convenía para adaptarlo a nuestro problema de minimización de costes. La elección final tomada, que es la de adaptar un Algoritmo Genético, subclase perteneciente a las Estrategias Evolutivas, se justificaría con las siguientes razones:

- Es un problema fuertemente No Lineal. La cantidad de variables que están interaccionando en el sistema de ecuaciones de un sistema de energía para tracción, hace inviable la aplicación de cualquier técnica de optimización Lineal, no tan solo por la dificultad de modelar sino por la dificultad intrínseca de estar trabajando con un sistema No Lineal.

- Problema difícil de aplicar a sistemas deterministas. Es muy complejo llegar a conseguir que todas las ecuaciones que componen un sistema ferroviario puedan llegar a tener un análisis por derivadas u obteniendo un gradiente final.

- En relación con lo anterior es importante la interrelación que se pudiera dar con un simulador ferroviario, que aportara la información que difícilmente se pudiera conseguir por medio de un complejo sistema de ecuaciones que modelara todo el comportamiento que se desea optimizar. Aprovechar la capacidad de Citef en este tipo de herramientas y desarrollar una nueva que ayude y se compagine con el optimizador, era otro de los objetivos.

- Problema multiobjetivo. El problema que se planteaba desde un principio era obviamente la optimización de costes, pero había otro problema que era cómo compaginar el modelo, que tenía por una parte la minimización de costes en los elementos de instalación del dimensionamiento eléctrico y por otra parte el problema de analizar los costes derivados de la explotación de ese diseño del sistema de energía de tracción. Dos objetivos contrapuestos, además del conjunto de restricciones que es necesario que no viole el nuevo sistema óptimo.

- Buena respuesta en cuestión de complejidad temporal. Aun teniendo cada vez máquinas más potentes, es obvio que no siempre se puede acceder a una supercomputadora para analizar un modelo. El flujo de trabajo normalmente pasa por un computador que tenga capacidad menor que una supercomputadora o computación paralela. La idea es que no haya una limitación a la hora de ejecutar el sistema experto planteado, y que un ingeniero en su puesto de trabajo o en su portátil, tuviera en un tiempo razonable la respuesta de la optimización.

- Adaptabilidad a la modelización del problema. Uno de los aspectos a tener en cuenta es la obtención de resultados con un modelo lo más sencillo posible. Los 
algoritmos genéticos son fácilmente adaptables a los modelos que se quieren optimizar, dotando de esta manera de una herramienta maleable y que no requiere una fase de análisis y diseño excesivamente compleja. Dentro de esta propiedad de fácil adaptabilidad, se puede añadir la capacidad de trabajar con variables de distinto tipo (binario, real, entero).

Los puntos anteriores dirigieron la elección hacia modelos basados en Algoritmos Genéticos. Se trataba ya de buscar cual sería el más adecuado de entre la vasta cantidad de ellos surgidos en los últimos tiempos. Finalmente se llegó a la conclusión de que sería buena la aplicación de más de uno, y que con ello se pudiera observar, contrastar y evaluar los resultados de cada uno de ellos. Los tres elegidos fueron:

- NSGA-II (Non-Dominated Sorted Genetic Algorithm II)

- AMGA-II (Archive-Based Micro Genetic Algorithm II)

- $\quad$ E-MOEA (Epsilon Multi-Objective Evolutionary Algorithm)

Los tres algoritmos son evoluciones de algoritmos precursores, cada uno de ellos tienen particularidades que en los apartados siguientes se van a tratar. La idea es exponer una introducción de cada uno de ellos. El desarrollo de la programación se ha realizado en $\mathrm{C}++$, integrándolo con el resto del código necesario para interactuar con el simulador y con las distintas funciones de evaluación, recopilación y codificacióndecodificación de los genotipos del modelo.

\subsubsection{Algoritmo NSGA-II. Aplicación.}

El algoritmo en cuestión se implementó pensando en una mejora del ordenamiento de los individuos de la población, mejora que se traduciría en una complejidad temporal menor, y por tanto menos tiempo en la obtención de resultados. El algoritmo NSGA-II [38], tiene un par de características primordiales:

- Aproximación a la ordenación rápida de los elementos no dominados

La primera parte del algoritmo va a hacer un barrido sobre todos los individuos que componen la población. La complejidad temporal total, baremo para determinar la capacidad de computación de los algoritmos [39], de esta parte del proceso es $O\left(M N^{2}\right)$. Primero se trata de ver la dominancia entre individuos. Para ello se comparan entre pares, de modo que si $p$ domina a $q$ (el individuo $\mathrm{p}$ es menor/mayor que q en los dos valores objetivo), este último se inserta en la lista de dominados de $p$ y el contador de individuos dominadores de $q$ aumenta en $1, n_{q}=n_{q}+1$. Esto se debe hacer para todas las tuplas de individuos posibles.

Una vez finalizado este primer paso, lo siguiente es ver en qué ranking se sitúa cada individuo. Para esto es muy útil el parámetro $n_{q}$, del que ya se ha comentado antes que indica el número de dominantes que tiene. Por tanto, si es igual a 0 , el individuo estará en el ranking 1, y así sucesivamente. Finalmente se tienen todos los 
individuos organizados por conjuntos, y cada conjunto representa un ranking de dominancia.

- Preservación de la diversidad de la población

Uno de los avances respecto al algoritmo predecesor NSGA (Non-dominated sorted Algorithm) [40], es la mejora de la función de compartición $\left(\sigma_{\text {share }}\right)$. La capacidad de mantener una variedad lo suficientemente amplia de soluciones teniendo en cuenta este parámetro, depende en exceso del valor que se le asigne y además, la complejidad temporal asociada para el cálculo era bastante alta. Por tanto el algoritmo NSGA-II, trata de evitar tanto que la parametrización dependa de un valor dado, como de mejorar la alta complejidad temporal anterior. Esto lo soluciona poniendo en marcha las ideas siguientes:

○ Estimación de la densidad de la población. El algoritmo para cada individuo, crea un área entre los dos vértices o individuos más cercanos al que se está estudiando. Una vez que se tiene el área, se calcula la distancia media del área del cubo creado. Esto sirve para identificar claramente los individuos que estén en los límites de cada función objetivo, ya que tendrán valor infinito en este indicador de distancia. Este parámetro también da información acerca de lo homogéneos o no que son los valores pertenecientes a cada función objetivo. Por último, la complejidad temporal para calcular los datos es $O(M N \log N)$.

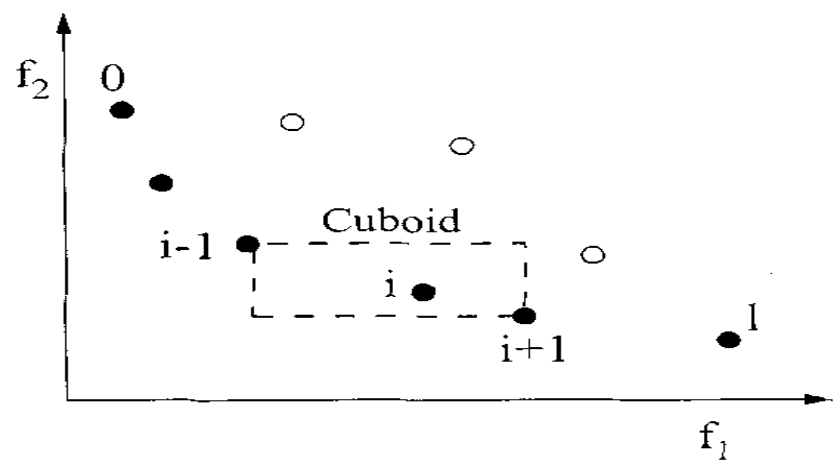

Figura 38 Explicación formación cuboide para cálculo compartición (Figura extraída de [38] )

○ Operador de selección "Crowded-Comparison Operator. Una vez que ya se han calculado todas la "crowded-distances", el siguiente paso es ver cuánto de buena es cada solución, dependiendo del valor obtenido. Tanto este operador como los cálculos hechos en el punto anterior, donde se asignaba un ranking a cada individuo, va a servir para ordenar individuos con mismo nivel de dominancia. Es decir será mejor el individuo que o bien tenga mejor ranking, o bien teniendo el mismo, la "crowded-distance" calculada es mayor que la del otro individuo, es decir la que está en una zona menos densa y por tanto, tiene más diferencias que los otros, lo que conlleva que se premia la diversidad en 
contraposición a la homogeneidad. Esto criterio intenta evitar la tendencia del algoritmo anterior de convergencia hacia óptimos locales.

La nueva generación por tanto se irá formando por los $n$ individuos con mejor ranking y en el caso que llegar a un nivel de ranking en el que son el mismo, se usaría el nuevo operador de densidad. Los $n$ individuos marcan el tope, al alcanzarlo, se habrá completado la nueva generación, y los individuos restantes se desecharán.

Por supuesto las restricciones también están incluidas en el algoritmo, de modo que si existiera alguna violación de alguna de las restricciones impuestas, entonces sería lo más determinante a la hora de pensar en no tener en cuenta al individuo en cuestión para la próxima generación.

La evolución de las soluciones finales y por tanto del Frente de Pareto final, cómo se llega al mismo y cómo se va trabajando con los distintos parámetros, y la incidencia de cada uno de ellos en el desarrollo, se va a explicar mejor conjuntamente con las pruebas realizadas y que se mostrarán en capítulos posteriores. Evidentemente los parámetros de cruce, mutación, distribución y demás tienen un peso e importancia que es intrínseco a la propia naturaleza de cada uno de ellos, pero se hace más claro a la luz de los resultados obtenidos.

Como es evidente, es un problema de programación global multiobjetivo, y por tanto es necesario desarrollar una librería o programa para poder ser interpretado por el ordenador. En este caso se ha optado por el lenguaje de programación $\mathrm{C}++$, que contiene funciones para interactuar con el programa matemático de cálculo matricial Matlab, con el que se realizan los cálculos del algoritmo eléctrico. Para los demás algoritmos también se ha seguido con este patrón de funcionamiento.

\subsubsection{Algoritmo épsilon-MOEA. Aplicación.}

El objetivo principal de este algoritmo es mejorar la diversidad de las soluciones planteadas por algoritmos anteriores, y en concreto por el steady-state MOEA (Multiobjective evolutionary algorithm).

En [41] se explica el algoritmo, aunque tiene referencias a otros para explicar conceptos, o bien el decisivo de épsilon-dominancia o el de operador de mutación polinomial.

Una de las características principales del algoritmo es que divide el espacio de soluciones en hipercubos. Esta división la crea usando el concepto de épsilondominancia. El uso de este valor parametrizable implica que el área de los cubos en los que se puede encontrar una solución, no va a ser mayor que lo que se pondere este parámetro. Es una forma de discretizar el espacio de soluciones y de dotar al sistema de un margen de error. Esta discretización implica que no puede haber más de una posible solución en el hipercubo creado, y por otra parte el error quiere decir que la solución no puede rebasar este parámetro épsilon valorado inicialmente. 
Para analizar los distintos individuos y el avance del algoritmo, se encapsulan los individuos en dos conjuntos diferenciados. Por una parte la población que se va creando del algoritmo evolutivo, y por otra parte se almacenan en un archivo las soluciones épsilon-no dominadas.

En la primera etapa del algoritmo se genera la población inicial de forma aleatoria, llamada $\mathrm{P}(0)$. Para la población del archivo, denominada como E, se asignan en la población inicial aquellos individuos épsilon-no dominados de la población $\mathrm{P}(0)$. Después, se escoge una solución de cada uno de los conjuntos. Del conjunto $\mathrm{P}$, se cogen dos individuos aleatoriamente y el más dominante es el que gana la selección. Del E, se coge uno aleatoriamente del mismo modo. Para crear la generación siguiente (offspring), con los individuos de cada conjunto escogidos, y una vez hallados el valor asociado al fenotipo del individuo (fitness), se calculan su array de identificación (B):

$$
B_{j}(f)=\left\{\left|\left(f_{j}-f_{j}^{\text {min }}\right) / \epsilon_{j}\right|\right.
$$

Ecuación 4. Array de identificación perteneciente a épsilon-MOEA

El operador de mutación polinomial es el que hemos usado para este MOEA. Este array discretiza las soluciones en áreas determinadas por el $\epsilon_{j}$. Este operador es el que se usa para las comparaciones entre individuos de la nueva población recolectada entre el conjunto $\mathrm{P}$ y el conjunto E, y se compara con la población del archivo de no dominados (E). El individuo que esté en un hipercubo mínimo es el que tiene mejor factor de evolución, por tanto el que se queda. Si hubieran dos individuos comparados que estuvieran en el mismo conjunto $B$ o índice de identificación en el array ( $i a)$, entonces se calcula el que tenga menor distancia euclidea, respecto del mínimo en el vértice del hipercubo que comparten. La decisión final implica que solo sobrevive uno para la siguiente generación. Con esto se preserva la diversidad y la unicidad por cubo. Además con esto los límites superiores no hace falta que estén parametrizados, ya que el barrido propio de cálculo de los $i a$, hace que se deban cubrir estos límites.

Una vez examinado el algoritmo, se pensó que podía entrar en el conjunto de métodos a implementar y que sería interesante contemplar cómo se comporta ante mismos problemas planteados respecto a los otros dos algoritmos.

\subsubsection{Algoritmo AMGA-II. Aplicación.}

Amga-II es un intento de mejorar los tiempos dedicados al análisis de distintas soluciones. Los tiempos computacionales, en la mayoría de Algoritmos Genéticos, está relacionado con los operadores básicos, es decir cruce, selección, mutación [42].

Otro de los motivos por lo que los autores deciden investigar en una nueva mejora para el algoritmo AMGA [43], es la posibilidad que ofrece al usuario de que con tan solo decir qué restricciones, funciones objetivo y soluciones finales quiere, no necesitaría emplear el tiempo que normalmente se tarda en configurar correctamente los parámetros para cada algoritmo y problema que se esté trabajando. 
En realidad lo que hace el algoritmo es ir acumulando en un archivo externo las soluciones no dominadas. Conjuntamente el algoritmo va a contener una población muy pequeña, consiguiendo que las funciones evaluadas no sean muchas. Al final se obtiene un frente de Pareto muy extenso, al ir almacenando soluciones en el archivo externo.

Una mejora respecto el predecesor AMGA, es que con la nueva versión, el algoritmo se asegura que se examinen los extremos de la diversidad poblacional. Antes, se hacía un torneo entre los integrantes de la solución no-dominada con los dominados, entonces se diluían los no-dominados.

Modifica la función de crowding distance, respecto al concepto que se describía en el NSGA-II, tal y como se explicará más adelante.

Creación del mating pool. El concepto de mating pool en algoritmos genéticos hace referencia al subconjunto a partir del cual se generarán todos los individuos que compondrán la siguiente generación. El algoritmo incorpora para ello el operador de cruce llamado DE (Differential Evolution) [44]. Para ello usa 4 padres (1 primario y 3 auxiliares). Cuando finaliza la operación de cruce, se habrá generado una sola nueva generación.

El algoritmo se explica como una sucesión de pasos a realizar, cuyo esquema general se resume así:

\section{Paso 1 Inicio}

Paso 2 Generar Población inicial

Paso 3 Evaluar Población inicial

Paso 4Actualizar el archivo, aplicando solo los individuos de la población inicial.

Paso 5 Repetir:

Paso 6 Se crea la Población Padre usando la población del archivo

Paso 7 Se crea el mating pool, teniendo como repositorio para ello tanto la Población Padre como el archivo.

Paso 8 Una vez conseguido el mating pool, se obtiene la siguiente generación aplicando los operadores de mutación y cruce.

Paso 9 Se evalúan los individuos de la nueva generación

Paso 10 En este punto del proceso, se puede actualizar los componentes del archivo, usando los resultados del Paso 9.

Paso 11 Mientras no se alcance la condición de finalización 


\section{Paso 12 Se pueden recoger los resultados del archivo y examinar las soluciones propuestas.}

\section{Paso 13 Fin}

Con el algoritmo presentado en pseudocódigo y por tanto, con unas nociones generales de cómo va a trabajar, a continuación se describen con más detalle los diferentes pasos que componen AMGA-II.

Pasos 1-5: Generación de la población inicial. Los autores eligieron usar para esto, el Latin Hypercube, mostrado por Loh [45] conjuntamente con el método de búsqueda imparcial de Knuth, hacen que la población sea distribuida de una forma aleatoria. Los conjuntos quedan encuadrados en compartimentos idénticos en cuanto al tamaño en el espacio de soluciones. Se trata de dividir $\frac{\mathrm{u}_{\mathrm{i}}-\mathrm{l}_{\mathrm{i}}}{\mathrm{N}_{0}}$, donde $\mathrm{u}_{\mathrm{i}}$ es el valor máximo para la variable $\mathrm{i}, \mathrm{y} \mathrm{l}_{\mathrm{i}}$ es el valor mínimo. $\mathrm{N}_{0}$ es el tamaño de individuos de la población inicial. De esta manera se trata de cubrir el espectro máximo posible de posibles soluciones. El siguiente paso es evaluar los distintos individuos que componen la población inicial.

Paso 6: creación de la población padre. En este punto aparece una desviación clara respecto del algoritmo NSGA-II. Mientras que en el NSGA-II toda la población puede actuar como "padre", en el AMGA-II, se busca como padre los de ranking 1, evitando así muchos procesos de cruce, selección y mutación. Además con la idea de diversificar el espectro de soluciones, se tratará de trabajar con aquellos genotipos que tengan un espacio mayor sin vecinos, dentro del ranking 1.

- Modificación del crowding-distance (CD). El objetivo es que el cuboide que se genera en este paso, tenga como punto central el genotipo en cuestión, en vez de estar en un extremo y la dependencia del cubo no sea mayor con los vecinos que con el genotipo mismo que se está estudiando para la creación del cubo. (M es el número de objetivos)

$$
C D(B)=\sum_{i=1}^{M} l_{i} r_{i}
$$

Ecuación 5. Modificación del operador crowding-distance para algoritmo AMGA-II

- El valor del crowding-distance va a posicionar las soluciones dentro del espacio, y se va a poder tener un baremo para determinar la diversidad de las mismas. De modo que analizando los vecinos que tenga y la distancia a la que se encuentren, se podrá manipular y cuantificar la diversidad que efectivamente tienen asignada.

Paso 7-9: creación del conjunto de apareamiento. No se genera por torneo binario clásico, como en el predecesor y en el NSGA-II, sino que se recupera el método Differential Evolution (DE) como operador de cruce. El procedimiento implica que 
primero se escoge uno de la población padre y para contrarrestar con tres individuos del archivo de soluciones.

Para la mutación, que la efectuaremos después del cruce, se ha usado el operador polinomial de mutación. El operador de mutación tiene un par de parámetros que es necesario conocer para darles un valor que permita la diversidad y la convergencia que se quiere. Estos valores, según se aporta en [42], dependen de cada problema de optimización aplicado.

Paso 10: Actualización del archivo solución. Inicialmente como se ha comentado, el archivo está vacío hasta que se inicializa la primera población, que entera pasa a ser del archivo de soluciones, solo las de ranking 1.

En las siguientes generaciones, dependerá de la función creada por los autores para la diversificación de la nueva generación. Objetivos son tanto la diversidad como mejorar el tiempo de convergencia, por lo que los autores integraron una búsqueda de los individuos a perpetuar, usando concretamente un método de poda de soluciones masificadas, basada en el método eficiente de vecinos más cercanos. En definitiva se trata de calcular la distancia euclidea con su segundo vecino más cercano. El que esté más cerca se elimina, en caso de ser la misma, se buscaría el tercer vecino más cercano y así sucesivamente. En caso que se compare con un extremo del frente, entonces la otra solución siempre sale perdiendo. El proceso seguirá hasta completar el número previsto para el archivo de soluciones no-dominadas. Este proceso es el que mayor complejidad temporal tiene $O\left(N_{a}^{2} \log N_{a}\right)$, siendo $N_{a}$ el tamaño de la población del archivo.

Para calcular el total de la complejidad temporal que tiene el algoritmo hace falta saber el número de evaluaciones que se va a practicar. Depende de las $\mathrm{T}$ funciones de evaluación, que es un parámetro de configuración, entonces el número de generaciones viene determinado por:

$$
\frac{T-N_{i}}{N_{p}}
$$

Ecuación 6. Índice para cálculo de complejidad temporal en AMGA-II

Siendo $N_{i}$ el número de individuos de la población inicial y $N_{p}$ corresponde con la población padre. Sabiendo que en general $N_{i}$ es mucho menor que T, y que la actualización del fichero de soluciones es el proceso más costoso, la complejidad final sería $O\left(\frac{T N_{a}^{2} \log N_{a}}{N_{p}}\right)$.

Obviamente este algoritmo es más complejo de trasladar a código, pero aun así la variedad que propone es muy interesante, y en la evaluación de las pruebas y resultados obtenidos se explicará con detalle cómo se ha trabajado con este algoritmo, y las consecuencias que se han observado a partir de los cambios en los parámetros iniciales. Obviamente al igual que los otros dos algoritmos, se ha programado también en $\mathrm{C}++$, integrándolo dentro del conjunto del sistema experto. 


\subsubsection{Aplicación de conjunto de algoritmos genéticos. Comparativa.}

La inteligencia artificial es una ciencia relativamente nueva y que por supuesto, está ligada al auge de los sistemas informáticos, que apoyan fuertemente la implantación de estas técnicas heurísticas en su mayoría. El repositorio de caminos y técnicas es muy amplio. En nuestro caso el conjunto de algoritmos de optimización se ha constituido en tres. Los tres tienen una característica común de concepto, y es que son una evolución de una versión anterior. En otros aspectos se diferencian bastante más, pero la elección de este conjunto de algoritmos, entre otros aspectos ya explicados, se ha debido a la posibilidad de poder comparar su comportamiento y eficiencia en frente de problemas iguales.

La aplicación de los algoritmos genéticos, y en definitiva la configuración de los parámetros críticos y cuál debería ser el efecto que produzcan, se explicarán con el apoyo de los ejemplos donde se han aplicado. Para parámetros comunes a la mayoría de algoritmos de esta naturaleza, si se conocen las consecuencias:

- Población: cuantos más individuos compongan la población, más diversidad habrá y se ocupará más el rango de posibles soluciones. En contra está el tiempo de respuesta, ya que se tendrán que hacer más evaluaciones y aplicar más operadores de cruce y mutación.

- Generaciones: al igual que para la población, mayor número de generaciones implica mayor tiempo de finalización, pero con seguridad, la evolución también será mayor, salvo en el caso que la convexidad se haya alcanzado en una generación anterior a la final, y ya no haya margen de mejora.

- Probabilidad de cruce: Un factor alto de este valor, implica más operaciones de cruce entre individuos, mayor diversidad entre los individuos. En cambio si se tiene un genotipo o conjunto de genotipos con muy buen valor fitness, es probable que al cruzar genotipos entre si, obtenga peores individuos en las generaciones posteriores. También un valor alto provoca más tiempo debido a que se efectuarán más operaciones de cruce.

- Probabilidad de mutación: Un comportamiento similar al valor de cruce. Un valor alto de probabilidad de mutación implica más tiempo consumido, mayor diversidad pero con la probabilidad más alta de empeorar individuos óptimos y perder convexidad en la consecución de un óptimo general.

- Factor eliminación de subestaciones: Este es un factor importante ya que afecta al que es probablemente el elemento más decisivo en nuestras optimizaciones, la subestación de tracción. Un factor alto implica que se eliminarán muchas subestaciones en cada genotipo, y que en dimensionamientos eléctricos muy poco sobredimensionados, costará más hallar una solución viable. En cambio en situaciones de sobredimensionamiento aparente, es un factor muy interesante para aplicar, con un valor elevado, ya que permite eso mismo, examinar situaciones con menos dimensionamiento que el inicial y comprobar si también funcionaría, por lo que se llegaría a una solución más ajustada con menor tiempo. 
La aplicación de variantes en el uso de estos parámetros combinados con los ejemplos de los Capítulos 5 y 6, detallarán lo que se puede esperar de cada algoritmo y cómo trabajar con ellos en futuras optimizaciones.

El otro factor de relevancia que se debe tener en cuenta, es la comparación de los resultados obtenidos por los tres algoritmos. Es muy común en la literatura encontrar trabajos donde se aplican diversos algoritmos y se decide comparar la viabilidad o prestaciones de cada uno de ellos, o bien comparar con versiones anteriores del algoritmo utilizado y verificar la mejora de la nueva técnica.

En [46] para la obtención de una conducción ATO eficiente integran un algoritmo MOPSO. Para comparar la técnica escogida, utilizan el NSGA-II, aplicado en nuestra metodología. Las comparativas son muy interesantes ya que describen evaluaciones prácticas para realizarlas. La diversidad del Frente de Pareto, el ratio de error de soluciones que se dan como óptimas cuando no lo son, distancia euclídea entre generaciones, tiempos de computación para alcanzar la generación final o el número de soluciones obtenidas, son las comparativas que han integrado en el trabajo. Evidentemente aportan muchas claves para las comparaciones correctas de los algoritmos genéticos. En este caso, no se comparan dos algoritmos genéticos, ya que representan paradigmas distintos, pero sirve como guía para encontrar esos criterios claves para evaluar una técnica de optimización como las que se usan en esta Metodología. Otro ejemplo, se encuentra en [47], que con el mismo procedimiento, compara un algoritmo basado en enjambre de partículas, confrontado con un algoritmo genético. Aquí el interés radica de nuevo en la comparación entre distintas modalidades de estrategias evolutivas, donde cada una, según los autores, destaca sobre la otra dependiendo del problema y de los valores aplicados a los parámetros principales de configuración.

En el artículo donde se presenta el NSGA-II [38], una vez que se ha explicado el algoritmo, preparan una serie de pruebas para comparar con otros algoritmos y corriendo los mismos contra problemas analíticos puros. El proceso de encontrar el parámetro adecuado, se centra en parámetros tales como diversidad, tiempo de computación, soluciones finales obtenidas. Todos los resultados finales contrastados con los cambios de parámetros, por ejemplo, cuando aumentan el número de generaciones comprueban que la diversidad es mayor y que para el caso del NSGA-II, el resultado de búsqueda del óptimo es mejor que para el otro algoritmo.

Siguiendo con el enfoque anterior, muchas de las comparaciones se realizan cuando los autores deciden publicar un nuevo avance en las técnicas de optimización. En el caso de [48], presentan el algoritmo SPEA, muy utilizado posteriormente para este tipo de comparaciones. En esta ocasión, el problema de la mochila es el elegido para las comparaciones. Lo compara con otros 4 algoritmos evolutivos, también con las mismas premisas para acometer esta tarea que los comentados anteriormente.

Un trabajo más reciente y muy interesante es el que se encuentra en [49], donde los autores trabajan con 5 algoritmos evolutivos para comprender su funcionamiento y 
variaciones ante configuraciones distintas. Los autores llegan a conclusiones de cómo manejar correctamente los parámetros en función del algoritmo que se esté usado y la respuesta que va a tener en los resultados finales del algoritmo. Los problemas y modelos usados para estas pruebas, suelen ser de baja complejidad, o un sistema de ecuaciones sencillo. Los resultados deberían aplicarse a problemas de ingeniería, y por este motivo se ha considerado muy interesante dotar a este tipo de comparativas, un enfoque mayor y más aplicable a problemas reales y también con una dimensión del modelo mucho mayor y compleja.

\subsubsection{Conclusión.}

En definitiva, existen muchas comparativas entre distintos algoritmos de optimización, distintos tipos de problemas, con especificaciones muy concretas cada uno de ellos y con búsqueda de resultados distinta. El aprendizaje de todos estos estudios es que la variabilidad de la configuración va a causar un impacto determinado en el resultado final y que dependiendo del tipo de problema, de la complejidad y naturaleza del mismo y del tipo de dato y solución a obtener, las diferencias entre algoritmos serán más notables. En nuestro trabajo las comparativas se van a centrar en unos pocos parámetros, y los resultados se analizarán en función de la diversidad obtenida, de la región de espacio de soluciones, así como de cantidad de soluciones finales en Frente de Pareto y de evolución de la población a lo largo de las generaciones. 


\subsection{Discretización zonal y ponderación de costes}

En el diseño inicial de un proyecto ferroviario se han de examinar múltiples opciones acerca de diversas áreas relacionadas con la infraestructura que se va a construir. En esta Tesis se ha querido introducir la posibilidad de que el encargado de diseñar el sistema eléctrico de tracción, a partir de ahora será denominado como SET, pudiera tener en cuenta algunas de esas opciones, y por supuesto, sus consecuencias. Cuando se habla de otros aspectos, en realidad se quiere referir a los problemas que aparecen, teniendo en cuenta la localización de la infraestructura ferroviaria y a los costes presupuestarios de los elementos básicos que van a componer el sistema de aporte de energía para las necesidades de la infraestructura.

No siempre se tiene disponible la mejor tecnología para implantar en los proyectos, más bien siempre se trata de llegar a un compromiso entre la calidad, que lógicamente debe tener un proyecto ferroviario, y el presupuesto final que se vaya a destinar para llevarlo a cabo. Nuestro objetivo es que con las soluciones propuestas por el sistema experto, el diseñador tenga mucha más información de cómo llegar a ese compromiso calidad-presupuesto. Por ello será necesario dotar al sistema de la capacidad de analizar costes, y al diseñador de cómo ponderar los costes según el elemento que esté tratando (subestación de tracción, catenaria, ...)

Aparte de los costes materiales de los elementos básicos, una infraestructura ferroviaria está afectada por el entorno donde se está construyendo. Existen zonas de principal vulnerabilidad debido a su coste medioambiental, dificultad de instalación, fácil/difícil conexión con las acometidas de la red de distribución y zonas donde son más complejas las tareas de mantenimiento. El resultado final deberá tener en cuenta situaciones en las que el usuario quiera o necesite evitar alguna zona especialmente crítica del tipo que se ha comentado. Para ello, y para que pueda ser interpretado por el sistema experto, se ha pensado en una Discretización Zonal, para poder introducir datos relativos a estas zonas y que puedan interpretarlos la herramienta de optimización.

En los siguientes apartados se va a comentar la conexión existente entre los tipos de zona estudiados y el dimensionamiento eléctrico. Finalmente se dará a conocer la Discretización Zonal y cómo se pretende realizar la ponderación o evaluación de los costes de cada elemento involucrado.

\subsubsection{Instalación}

La instalación de SET comprende muchos elementos y construcciones básicas, obras a realizar y trabajos en campo. Todos los elementos no tienen la misma trascendencia a la hora de realizar estudios de análisis del sistema de energía. La concreción en estos casos también es aplicable, y los elementos que tenemos en cuenta en nuestros estudios son los señalados en el Capítulo 2:

- Catenaria

\section{- Subestaciones}




\section{- Autotransformadores}

\section{- Zonas Neutras}

\section{- Seccionadores y enlaces}

Estos son los elementos clave en la mayoría de estudios de validación, optimización y dimensionamiento de sistema eléctricos que se llevan a cabo. Es importante volver a destacar que dependiendo del tipo de sistema eléctrico que se vaya a implantar, el conjunto de elementos será distinto, y también las características de cada uno se tendrán que adaptar para que el sistema llegue a funcionar correctamente.

\subsubsection{Mantenimiento}

En cada proyecto se examinará la posibilidad de incluir zonas de especial atención respecto al impacto que puedan tener los trabajos de mantenimiento, o material a estudio para incluir, que tenga un sensible impacto en los costes generales del proyecto.

\subsubsection{Conexión con la red general}

La relevancia de situar las subestaciones y autotransformadores lejos de la toma de la red general es alta, en mayor medida cuando los costes de instalación de la conexión deben pasar por un proceso de obra civil costoso. En el Capítulo 3, se resume la importancia de que este factor esté vinculado.

\subsubsection{Medioambiental}

Por último destacar que en la discretización zonal, que se analizará en el apartado siguiente, tiene cabida el impacto medioambiental que puede llegar a tener el sistema de electrificación sobre su entorno. En muchos proyectos no se trata solo de mejorar los costes asociados, sino que en este caso incluso es obligatorio tener en cuenta las recomendaciones de no violar zonas medioambientales, en función del pliego el proyecto en concreto.

\subsubsection{Discretización zonal}

Durante el planteamiento inicial de la metodología de optimización del diseño eléctrico, se pensó dar cabida a las consecuencias que tienen los sobrecostes provocados por un diseño que no tuviera en cuenta los costes relacionados con los etapas de ingeniería comentados en los apartados anteriores y también en zonas medioambientales, con difícil justificación de la apropiación de estos terrenos para el proyecto ferroviario.

En la figura se han distribuido, a modo de ejemplo, cómo va a ser el planteamiento de trabajo para incluir los tipos de área/zona que se ha comentado dentro de las ecuaciones del sistema experto. 


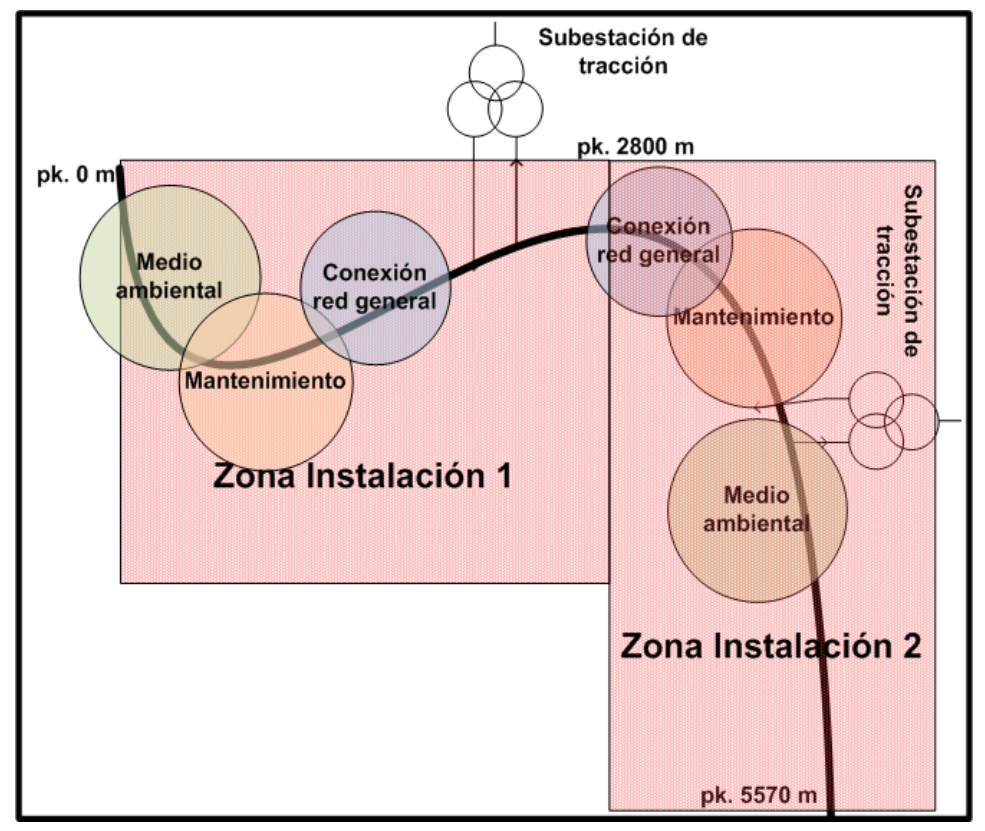

Figura 39 Ejemplo ilustrativo distribución zonas

\subsubsection{Zonas de impacto exterior}

El primer enfoque importante deriva hacia la manera de incluir espacialmente las superficies cubiertas por las zonas. En cualquier estudio previo de localización de este tipo de zonas, seguramente el área representada por cada una de ellas no sería, como en el ejemplo, circular. Por este motivo se ha diferenciado el tratamiento dependiendo del tipo de zona que sea:

\section{- Zona con impacto medioambiental}

Estas zonas se reconocerán en la documentación relativa a la parte del proyecto dedicada a la obra civil, y representarán aquellas áreas que tengan un relativo impacto medioambiental. Se ha considerado que el impacto que se provoque pueda ser gradual a la importancia de la zona donde se desea construir o que pase la red de tracción eléctrica.

\section{- Zona con conectividad a la red de distribución}

La toma de la red de distribución de media/alta tensión se posiciona en un lugar poco accesible desde el área que se ha establecido para este tipo. En este caso, el ingeniero según las acometidas de la red general, el estudio de los planos de la línea y los puntos posibles de conexión a esas acometidas, configurará la superficie correspondiente.

\section{- Zona de mantenimiento}

En relación al tipo de mantenimiento que se prevé se vaya a realizar cuando la infraestructura esté ya en fase de operación, el ingeniero deberá evaluar qué zonas tendrían un impacto importante en caso de tener que efectuar acciones de 
mantenimiento en ellas y en función de la importancia del tipo de mantenimiento que se prevé se va a llevar a cabo durante la vida útil del sistema ferroviario.

La identificación y localización exacta de las zonas de estos tres tipos que estén dentro del recorrido del sistema de toma de energía es la primera parte del problema. Está definido dentro de las tareas iniciales del usuario del sistema experto, y como se ha detallado, a partir de la documentación se deberá realizar. La segunda tarea es trasladar estas áreas de influencia a las ecuaciones de función objetivo y restricciones que formarán parte del modelo de optimización.

El área o superficie que corresponde a cada zona normalmente tendrá una forma o contorno poco estructurado. Para que se pueda integrar dentro de las ecuaciones, se estudiará esa área y se procederá de la siguiente manera:

- Se calculará la intersección entre el área de instalación del sistema de energía correspondiente a la línea ferroviaria junto a las áreas de las zonas especiales que existan en cada proyecto.

- El trayecto de la línea se estudiará con los puntos kilométricos que tengan asignados en el trazado. Significa que para conocer el trayecto, la línea va a ser unidimensional.

- Por tanto cuando exista alguna intersección, solo se trabajará con longitudes, de forma unidimensional. Como ejemplo la siguiente figura, donde en rojo se destaca la longitud que se tendría en cuenta para los cálculos de aportación de las partes de las zonas involucradas:

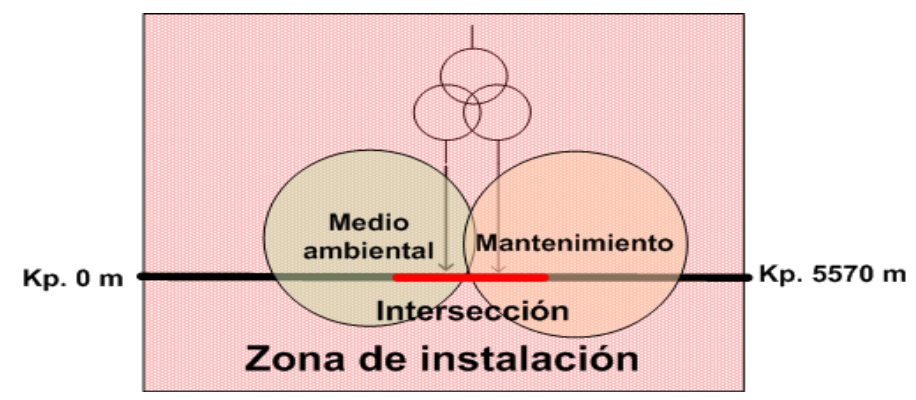

Figura 40 Ejemplo cálculo intersección de zonas

\subsubsection{Zonas de Instalación}

Para la instalación de los elementos que se han descrito en el Capítulo 2, era necesario idear un sistema que permitiera ubicarlos en cualquier posición a lo largo del trayecto definido inicialmente. Concretamente se trata de modelar los elementos que si tienen flexibilidad para modificar su posición, es decir, las subestaciones de tracción, autotransformadores, zonas neutras. El algoritmo heurístico debía ser capaz de buscar durante su recorrido por todas las posibilidades de posicionamiento para cada elemento.

La discretización zonal pretende alcanzar los objetivos necesarios. Se basa en: 
- Si se está ante un proyecto nuevo:

- Se calcula el máximo de subestaciones posibles a instalar, según las características iniciales y teniendo en cuenta la normativa.

- Si el proyecto está ya en funcionamiento:

- Ya se tienen el total de subestaciones. Según se prevea un análisis de optimización o bien, por problemas de suministro de energía, se optará por mantener el número de subestaciones o incrementarlo.

- A continuación se divide la longitud total de la línea entre el número de subestaciones de tracción total calculado.

- La discretización se completará al asignar una subestación a cada longitud resultante.

Cuando se explique cómo se van a calcular las posiciones posibles de cada subestación dentro de su parte discretizada correspondiente (apartado 4.5), se comprobará que el espectro de posibilidades está cubierto.

La intersección entre zonas se realiza teniendo en cuenta esta discretización. Dependiendo de la longitud que se haya establecido para la instalación de las $s$ Subestaciones, sabiendo que pertenece a su i-zona instalación correspondiente, entonces se comprueba con qué zonas de impacto exterior, comparte puntos kilométricos y se calcula el valor de tramo que coinciden. Ese tramo será el que se usará para calcular el valor atribuible a esa zona, en caso de instalar una subestación.

\subsubsection{Ponderación de costes}

El último paso es aplicar costes a los componentes y las zonas que van a componer cada diseño que se programe para encontrar un mínimo de los costes totales del proyecto.

Incluir los costes debe tener en cuenta los siguientes aspectos:

- Hallar los costes monetarios de todos los elementos que van a intervenir en el diseño y que se pueden introducir en el sistema experto.

- Costes asociados a zonas relacionadas con el apartado anterior que se vayan a incluir en el proyecto.

- Obtener una unidad de medida válida para los distintos costes y que sea homogénea.

El valor real de los elementos involucrados, no siempre va a ser posible conseguirlo. Es probable que dependiendo del nivel de involucración con el proyecto, se tenga acceso al presupuesto exacto de los elementos de distribución de la potencia de tracción, como de la catenaria. En un escalón inferior, en cuanto a relación cliente-empresa contratada, de trabajo directo con el estudio eléctrico, no es muy sencillo el acceder a 
estos datos. Por otra parte, el análisis de los costes derivados de introducir, por ejemplo, una subestación en una zona que tiene un coste medioambiental relativo, es una consecuencia que conlleva otras connotaciones y consecuencias, que no siempre tienen como principal motivo el presupuestario. En este último caso, se vería afectada en mayor o menor medida una parte de una zona protegida, por lo que es posible que el impacto debiera medirse no tanto en el presente, sino a largo plazo, ya que las infraestructuras ferroviarias tienen una vigencia e impacto con un intervalo de tiempo elevado.

Es importante también contextualizar en qué modelo se van a integrar los costes que se están relatando. El modelo de optimización es un modelo fuertemente no lineal y cuyas variables son continuas. Como se verá más adelante, no todas las variables tienen como unidad de medida el coste, en unidad monetaria. Por lo que se va a necesitar una unidad de medida adimensional para que pueda tener cabida en las ecuaciones tanto de la función objetivo como de las restricciones.

Una vez analizadas las posibilidades que se adaptarían al modelo de costes, se han considerado dos caminos factibles para solventar este paso:

- Determinar los costes exactos y reales de cada elemento y zona que va a intervenir en el dimensionamiento energético de la línea a estudiar.

- Ponderar cada elemento en función de una medida estándar aplicada a un componente/coste básico dentro del proyecto.

La primera opción se va a presentar en muy pocos estudios. No siempre se tienen todos los datos para evaluar al nivel requerido. Aunque el sistema está preparado para trabajar con la primera opción, la experiencia dice que la segunda opción es la que en la mayoría de casos se va a presentar.

La asociación peso-elemento eléctrico y peso-zona es una etapa que forma parte de la configuración del sistema experto que va a tener que hacer el diseñador o ingeniero. Los pesos asociados deberán tener en cuenta que:

- Niveles de coste entre componentes de mismo tipo.

Por ejemplo si se están ponderando los pesos de la subestaciones que van a poderse instalar en el sistema, aspectos como potencia que pueden suministrar, o por ejemplo si nos encontramos en un sistema de continua y la subestación es reversible [50], pues consecuentemente esta subestación deberá tener un peso mayor que otra que no lo sea o tenga grupos de rectificación de menor potencia. También por ejemplo si los alimentadores que van desde la subestación hasta la catenaria son de mejor calidad respecto de otra, sería otro caso a evaluar. En definitiva, en estos casos, la capacidad y calidad de los elementos de mismo tipo se han de definir y establecer un relación que refleje fielmente las diferencias entre la calidad de cada elemento que se desea implantar. 
- Niveles de coste para los distintos tipos de zonas integradas en el modelo.

En este caso se han de tener cuenta otros factores que se van a explicar a continuación.

- Ponderación zonas de instalación

Cada zona de instalación está asociada a la posibilidad de que una subestación en concreto se vaya a instalar en ella. En este caso, como se ha comentado en el punto relacionado, si se instala en una zona compleja, los pesos asociados van a tener que ser mayores, pero esto va a incidir directamente en la posibilidad de que la subestación que contiene pueda o no estar dentro del proyecto final.

- Ponderación zonas de conexión a red general

- Ponderación zonas de mantenimiento

- Ponderación zonas de impacto medioambiental

La relación entre todas las variables que componen el sistema se vincula a partir de ecuaciones. La formulación creada para modelar el sistema se muestra en los siguientes apartados. 


\subsection{Pico de demanda máxima de potencia. Situación estacionaria de análisis del Sistema de energía de tracción (SET).}

La tarea de modelar un sistema fuertemente no-lineal, implica que en algún punto se han de asumir simplificaciones de alguna parte del modelo. Estas simplificaciones hacen que el método de optimización pueda tener una respuesta temporal mucho más adecuada a las necesidades reales. En el desarrollo de la idea de este sistema experto surge una dificultad, y es cómo obtener en un tiempo razonable, el resultado de los distintos genotipos propuestos por los algoritmos genéticos. La solución que se ha adaptado se va a detallar en este apartado, de modo que se examinen tres vertientes posibles de trabajo, para finalmente conocer cuál es la que se ha implementado y el por qué.

\subsubsection{Pasos críticos para analizar un dimensionamiento eléctrico}

A pesar que se han iniciado conversaciones a nivel Europeo y trabajos de investigación para regular y controlar la fiabilidad de los simuladores eléctricos aplicados al ferrocarril, aún no se ha llegado a un consenso. La complejidad de los modelos que intervienen hace que no sea fácil determinar los límites de análisis para testear un simulador de este tipo. Los resultados obtenidos no dependen solo del algoritmo eléctrico y de su implementación, sino que dependiendo de la profundidad que tenga cada herramienta en áreas como material rodante o señalización, por ejemplo, se podrá verificar con mayor o menor exactitud el comportamiento simulado versus el real del sistema ferroviario.

Habiendo descrito el simulador Hamlet, con los módulos que tiene integrados, es importante mostrar cómo se suele desarrollar un estudio de dimensionamiento eléctrico, no ya para validación, sino para optimización del mismo: 


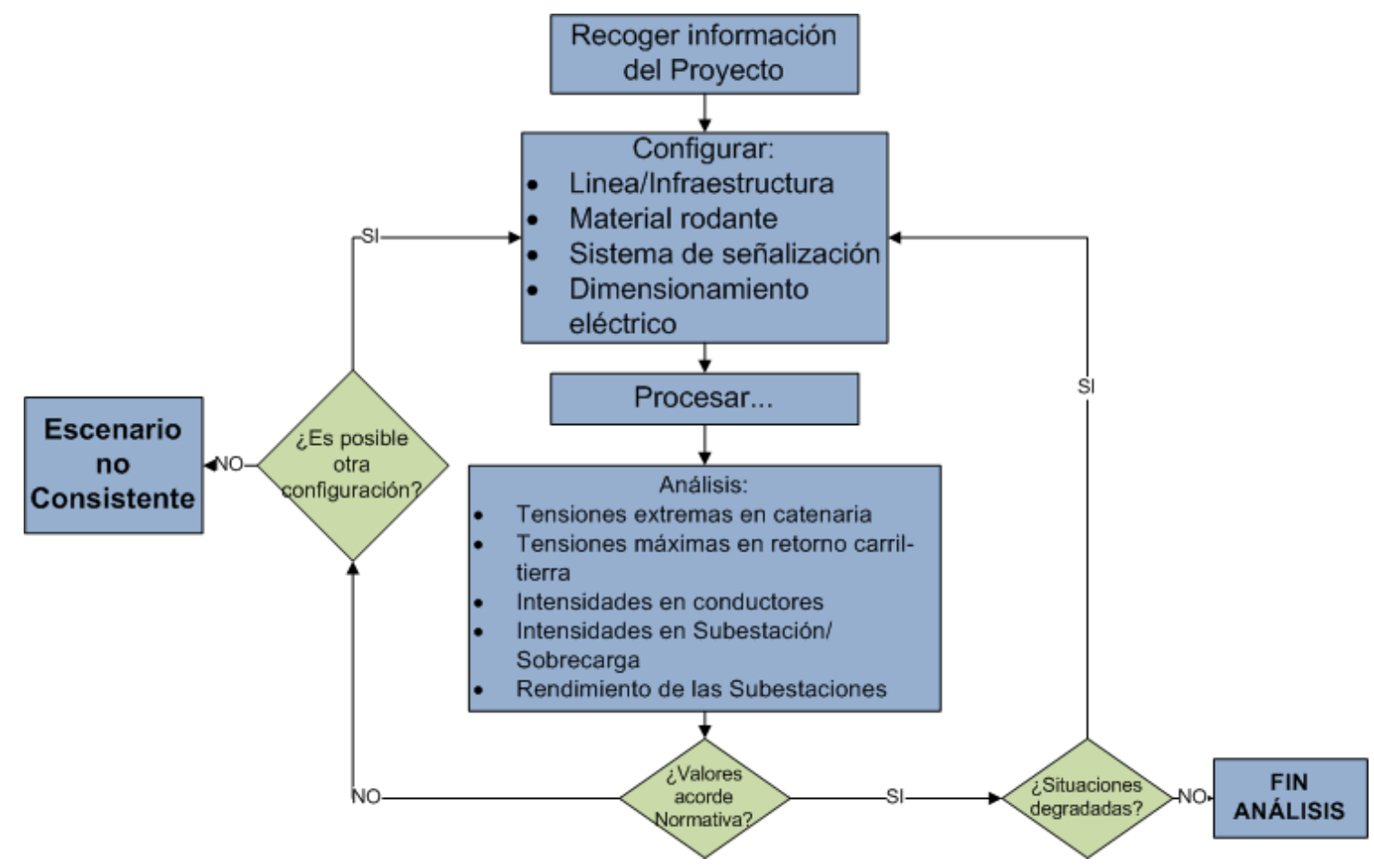

Figura 41 Diagrama proceso manual optimización dimensionamiento eléctrico

En la Figura 4 se distingue un núcleo claro. Este núcleo de actuación sería el formado por los subprocesos donde se configuran los módulos para volver a procesar o simular, y finalmente gestionar y analizar los resultados. En los capítulos posteriores de Resultados, tanto para corriente continua como para alterna, aparecen los tiempos de simulación para un escenario concreto. Además se tendrían que sumar tiempos de análisis y configuración, pero estos serían comunes a ambos métodos, el tradicional y el optimizado. 
La idea es llegar a tener un sistema automatizado representado en el diagrama de la figura siguiente:

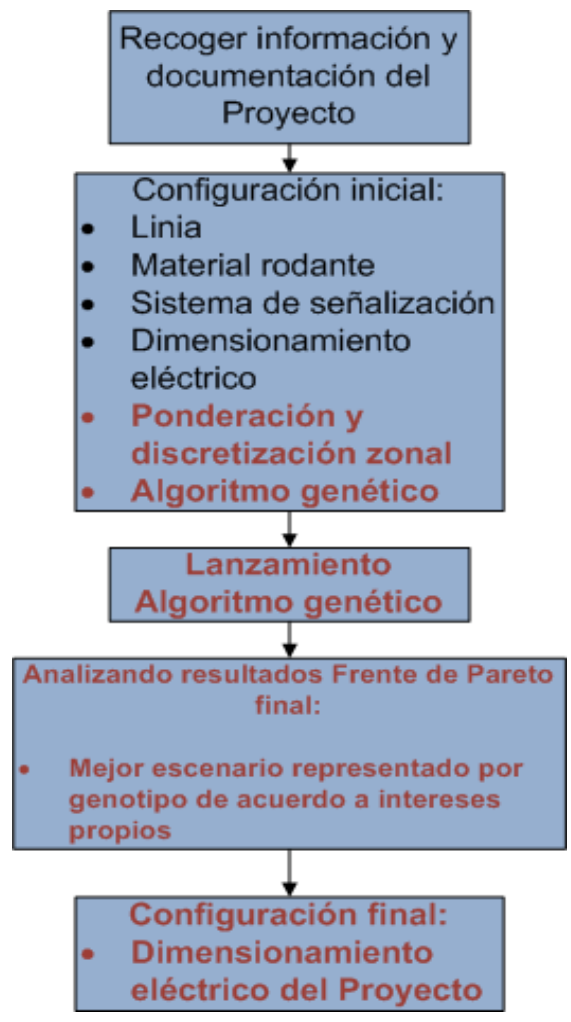

Figura 42 Diagrama de Optimización del dimensionamiento usando Sistema Experto

La tarea de configuración durante la automatización, se le asigna a la implementación integrada en el Hamlet que permite automáticamente reconfigurar. Esta implementación se originaría después de cada decodificación realizada para cada Genotipo.

La tarea de Lanzamiento de algoritmo genético trabaja ligado al Hamlet, como se ilustra en la siguiente Figura:

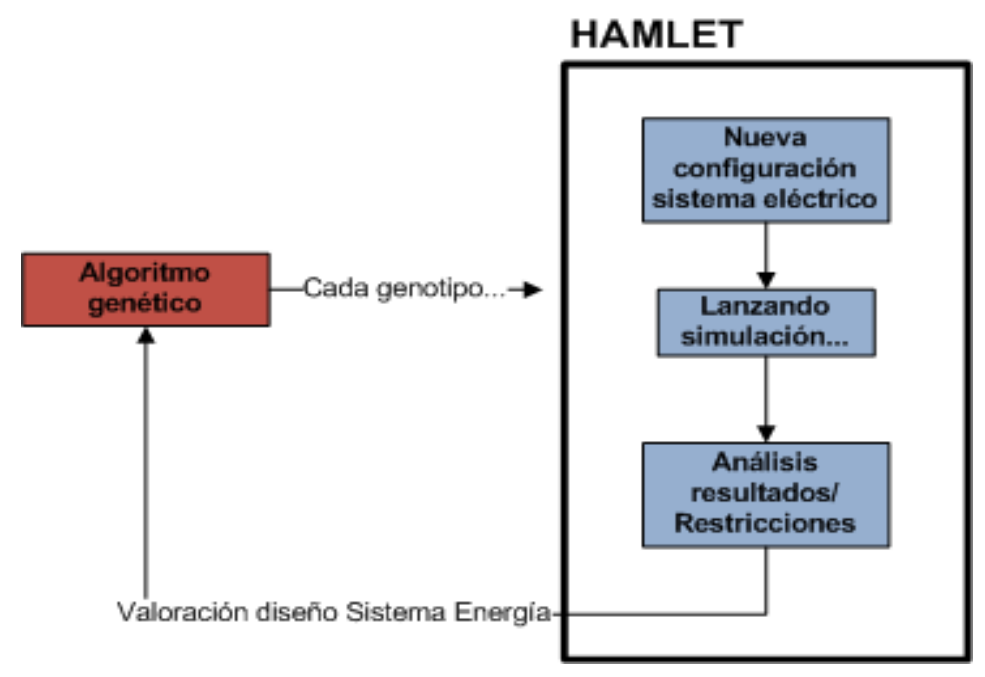

Figura 43 Interactuación Hamlet-Algoritmo genético 
La tarea de análisis de resultados es dependiente de la realización de una comprobación de no superación de límites que aparecen en la correspondiente normativa. Las subestaciones de tracción y la consistencia de la catenaria suelen servir como medida para conocer la bondad del SET.

A continuación se presentan los criterios a partir de los cuales se pretende elaborar una estrategia de conocimiento del SET, y que sirva para evaluarlo.

\subsubsection{Análisis de comportamiento de SET. Tensiones extremas en cables. Tensiones en retorno carril-tierra. Intensidad.}

Factor muy ligado al plan de explotación existente en la línea. En cada malla eléctrica formada por la subestación y los trenes que alimenta, existe una caída en la tensión que puede llegar a provocar una bajada inasumible para las necesidades del tren [51]. De igual manera se opera con las tensiones máximas que se inyectan en la catenaria. Este caso solo ocurre cuando los trenes además de freno reostático, pueden usar el sistema de frenado como generador de energía [52]. Si el sistema no está preparado convenientemente para absorber esa tensión generada o para volcarlo en la red general [53], se produce una sobretensión que de igual forma invalidaría el diseño del sistema de energía.

Una vez que las intensidades pasan por los trenes, la distribución de corriente suele dirigirse a los carriles o hilos de retorno. La regulación de los límites para las tensiones de retorno carril-tierra vienen en la a norma EN 50122-1 [54] a la hora de definir la tensión máxima admisible:

- Sistema eléctrico en contínua

- 120 Vcc permanente.

- 150 Vcc para intervalos de 5 minutos.

- 170 Vcc para intervalos de 1 segundo.

- Sistema eléctrico en alterna

- 60 Vcc permanente

- 65 Vcc para intervalos de 5 minutos

- 80 Vec para intervalos de 1 segundo

El paso de la corriente por los conductores de la catenaria provocan pérdidas debido a la resistencia que ofrece estos cables. Una de las consecuencias importantes es la caída de tensión. La otra es el calentamiento que produce en los cables, atendiendo a la Ley de Joule. Este calentamiento en los cables debe estar regulado para que no supere ciertos niveles que hagan un daño irreparable al cable, o que merme su capacidad gravemente. Los valores máximos de corriente por los conductores es otro de los parámetros importantes a tener en cuenta en los análisis de un SET.

Estas comprobaciones se realizarán para escenarios donde el funcionamiento de las subestaciones sea el deseado y también para situaciones donde falle un transformador de 
aquellas subestaciones que tengan más de uno, las llamadas situación degradada de funcionamiento.

\subsubsection{Análisis comportamiento en Subestaciones de Tracción. Intensidades máximas y medias cuadráticas. Estudio de sobrecarga.}

Dejando de lado las subestaciones de alimentación de la red de distribución, los elementos críticos llevados a examen suelen ser las subestaciones de tracción. Las intensidades de corriente que fluyen por la subestación están delimitadas por la intensidad nominal proveniente de los grupos de potencia que tiene la instalación transformadora/rectificadora. El análisis suele comprender tres etapas:

1- Analizar las Intensidades máximas

2- Analizar las intensidades medias cuadráticas. Estos valores suelen ser más significativos, ya que informan de un valor medio y se puede comparar con la intensidad nominal, pudiendo detectar posibles problemas si supera ese valor nominal.

3- Analizar los límites de carga. Se compara la curva de carga impuesta por la normativa UNE-EN 50329 [55], con los porcentajes para cada régimen temporal, con los calculados por las simulaciones.

Si alguna de estas métricas supera el límite permitido, el resultado final del análisis debería ser negativo, ya que existiría al menos una situación para la cual el sistema no sería viable.

\subsubsection{Análisis comportamiento en Autotransformadores. Intensidades máximas y medias cuadráticas.}

Para los sistemas eléctricos en alterna dual, los autotransformadores son fundamentales para que el sistema alcance la funcionalidad y las propiedades intrínsecas a este tipo de sistemas. En proyectos donde se trabaje con este sistema, los autotransformadores, al igual que las subestaciones, se verán expuestos a análisis de su comportamiento. Para ello se analizarán las intensidades máximas y sobre todo, de nuevo, las Intensidades medias cuadráticas.

Del mismo modo que en el caso de las Subestaciones, esta característica sirve para evaluar de forma eficiente el estado de los autotransformadores.

\subsubsection{Automatización del proceso. Motivación primordial y objetivo final.}

Finalmente hay que comentar el problema acerca de los problemas de automatizar la simulación. La resolución del dimensionamiento eléctrico de un plan de explotación requiere que en cada ciclo o paso de integración, se calculen las matrices que componen el algoritmo eléctrico. Con el simulador Hamlet, primero se calculan todos los ramales eléctricos por separado, obteniendo las impedancias que recorren los conductores. Como cada dimensionamiento puede ser distinto incluso a este nivel, no se puede descartar este paso en las siguientes simulaciones. Con las impedancias calculadas se 
pasa a resolver la malla completa, teniendo ya en cuenta las potencias demandadas, subestaciones, etc.. Estos cálculos debido a la dimensión de las matrices, proporcional al número de ramales o tramos eléctricos, hace que el tiempo de cálculo sea alto y que ralentice la obtención final de resultados. Esta es una barrera para el automatismo de la optimización del dimensionamiento eléctrico. Los costes temporales no serían asumibles para alcanzar el objetivo perseguido.

En estudios de optimización del dimensionamiento eléctrico, se suele buscar un camino para evitar este problema. En el trabajo de E. Pilo [56], la idea es usar una serie de fotos de la operación general del sistema ferroviario, que los autores consideran más crítica para el dimensionamiento. Acotando el estudio de ese número finito, acortan también el tiempo de evaluación. Otro grupo de trabajo, de la Universidad de Vigo [57] los autores calculan la demanda de potencia de un día para el tráfico de una línea que utiliza corriente continua.

La automatización del proceso exigía un criterio que permitiese acotar el rango de estudio y que fuese fiable, al igual que los trabajos anteriormente citados. Además debían tener en cuenta las características del sistema experto y de la información que trata. A continuación se relatan los caminos que se plantearon para conseguir una focalización del problema.

\subsubsection{Alternativas para conseguir punto de focalización consistente y fiable}

Las posibilidades que se han manejado para encontrar la forma de evitar el análisis completo de cada genotipo, se pueden ver en la figura siguiente:

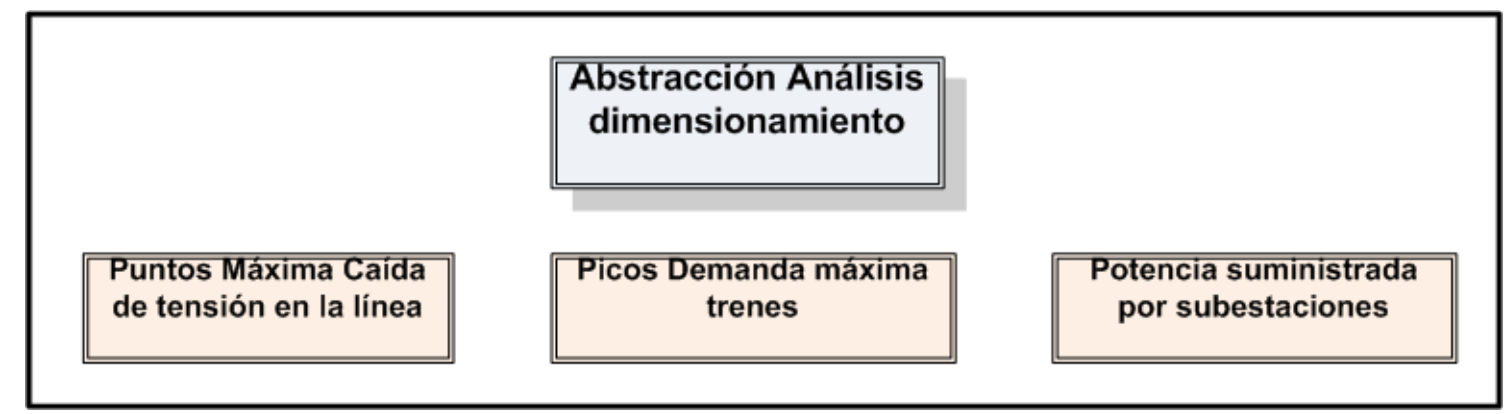

Figura 44 Estrategias focalización análisis dimensionamiento

\subsubsection{Selección puntos en la línea donde la caída de tensión es máxima}

La caída de tensión a lo largo de la línea, viene determinada por la impedancia calculada de los cables conductores de la catenaria y la impedancia en paralelo entre el grupo que conforma la catenaria. Esta impedancia hace que caiga la tensión desde la subestación o autotransformadores hasta los trenes que estén circulando. Por tanto la capacidad del tren se verá limitada siempre por esa caída de tensión final que captan por el punto de contacto del tren-catenaria. Aplicado al algoritmo de cálculo eléctrico del Hamlet, se debería calcular el instante de tiempo en el cual la caída de tensión ha sido máxima, y por tanto recoger de los resultados almacenados de las posiciones donde los trenes, punto pantógrafo-catenaria, tengan un sumatorio de caída de tensión máximo. 
Con esta solución existe un problema de inestabilidad. Se habría conseguido un baremo para calcular el dimensionamiento, pero al ser un criterio dependiente del diseño eléctrico, al realizar cambios de posición de los elementos de generación de energía, es muy probable que el paso de integración que había propiciado la caída máxima total, ya no fuera el que se estuviera examinando, y por tanto no es fiable.

\subsubsection{Potencia suministrada por las subestaciones de tracción}

La implementación en nuestro simulador de las subestaciones de tracción se pensó como una subestación de potencia infinita. Es decir, es un elemento del sistema de energía que proporciona la potencia necesaria a la red, independientemente de la carga que esté soportando o de la configuración interna de la subestación, de la que si se tiene en cuenta la impedancia, así como los alimentadores desde la barra de la subestación hasta el punto de contacto con la catenaria. El algoritmo eléctrico va a calcular la malla obteniendo los valores de parámetros eléctricos involucrados en la subestación. Uno de los parámetros calculados es la Intensidad/Potencia que genera la subestación, en cada paso de integración.

La inestabilidad de este criterio vendría dada por la nueva posición de la subestación y con la posibilidad de que en su área de influencia, en el ciclo escogido para el caso anterior hubiera un tráfico distinto del que existe en la nueva área. El algoritmo tendería a mejorar la situación en función de las necesidad de la subestación no del tráfico, por lo que premiaría aquellos escenarios donde el tráfico estuviera concentrado en las subestaciones de tracción, eliminando la mayor cantidad de subestaciones posibles, y por tanto seguramente habrían mucho casos de estudio a posteriori, que al analizarlos, resultarían con niveles de sobrecarga por encima de los límites permitidos. Al igual que para el criterio anterior, se descartó esta vía de trabajo, al no garantizar la estabilidad de las soluciones.

\subsubsection{Pico de demanda máxima de potencia de los trenes.}

El simulador tiene integrado el módulo de tracción, el cual tiene como misión vencer las fuerzas resistentes que actúan en contra del movimiento esperado y a la velocidad deseada:

$$
\text { PotTren }=\left\{\begin{array}{l}
\text { PotTraccion, si tren tracciona } \\
\text { PotFreno, si tren está frenando }
\end{array}\right.
$$

- Tracción

$$
\text { PotTraccion }=\frac{F m * s p}{\text { mechef }}+\operatorname{Saux}
$$

Ecuación 8. Cálculo de potencia demandada por el tren en tracción

- Freno

$$
\begin{gathered}
\text { PotFreno }=F m * s p * \text { mechef } * \text { regcoeff }+ \text { Saux } \\
\text { Ecuación 9. Calculo de potencia generada por el freno }
\end{gathered}
$$


donde:

PotTraccion es la potencia obtenida del cálculo de las fuerzas eléctricas de los trenes [W]

PotFreno es la potencia regenerada por el freno [W]

vel es la velocidad del tren $[\mathrm{km} / \mathrm{h}]$

Fm es la fuerza mecánica de los trenes [kN]

mechef es el rendimiento mecánico medidos respecto de la carga que obtiene del pantógrafo

Saux Potencia demandada por los sistemas auxiliaries de los trenes [W]

regcoeff Coeficiente de regenerabilidad al usar el freno como generador

Si el material rodante tiene la capacidad de usar frenado regenerativo, y si el tren que esté en tramo de frenado lo acciona sin usar el freno neumático, el criterio seguido es que toda la energía regenerada, después de alimentar los sistemas auxiliares, con la potencia que tengan configurada por defecto, se inyecta en la catenaria. Además, esa energía será aprovechada, o bien por otros trenes o inyectando de nuevo a la red general (subestaciones reversibles o directamente en caso de corriente alterna). Por tanto, la demanda final se calcularía con el balance de potencia:

$$
\text { PotDemanda }=\sum_{t=1}^{\text {ntrenes }} \text { PotTren }_{t}
$$

Ecuación 10. Potencia total demandada por los trenes en el plan de operación

La potencia demandada por los trenes tiene en cuenta todos los factores que afectan a la explotación de la línea. Además son valores que se calculan independientemente del sistema eléctrico que se haya instalado, o que esté en funcionamiento en momentos precisos. Con este criterio se pretende subsanar las deficiencias que tenían los otros dos; el algoritmo no va a buscar un dimensionamiento para mejorar la estabilidad y costes del mismo, sino que buscará el mejor sistema para aportar la energía necesaria al tráfico existente.

\section{- Situaciones normal de funcionamiento}

Se denomina funcionamiento normal del sistema eléctrico, cuando no existe ningún fallo en ninguna subestación de tracción que haya instaladas en la línea, o que estén involucradas en la generación de energía para el plan de explotación de la línea.

A lo largo de todos los pasos de integración de la simulación mecánica del plan de explotación se examina el valor de PotDemanda. Al final del proceso, el sistema se va a quedar con el instante de tiempo donde esa demanda ha resultado mayor en valor neto. 


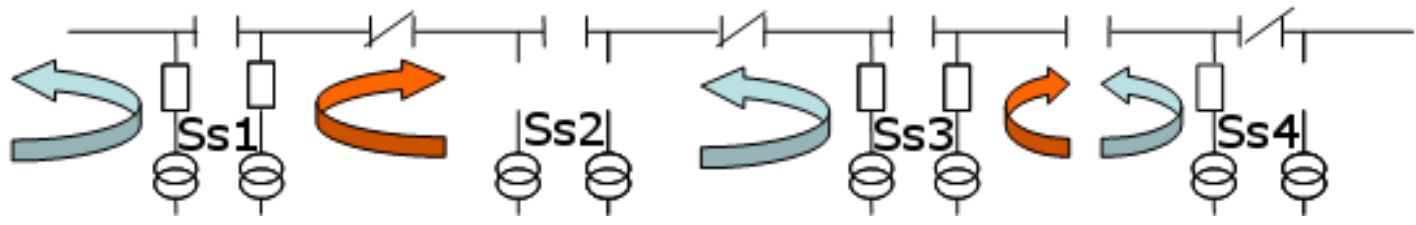

Figura 45 Ejemplo explicativo situaciones degradadas en sistema eléctrico ferroviario

En la Figura anterior se ha puesto un gráfico ilustrativo del funcionamiento del sistema ante situaciones degradadas del mismo. El comportamiento descrito es el que se suele adoptar en la mayoría de casos:

- Situación degradada simple: Cuando una subestación de tracción está compuesta de más de un grupo transformador/rectificador, y uno de estos grupos se ha averiado o ha dejado de funcionar, el escenario pasa a funcionar en situación de degradada simple.

- Sistema en corriente continua: Normalmente cuando ocurren este tipo de averías, el funcionamiento del sistema sigue inalterado, es decir no hay ajustes ni del sistema eléctrico ni de operación en la línea.

- Sistema en corriente alterna: Este caso se corresponde con el de la Figura Subestación 4, y normalmente se cierra el seccionamiento de separación, en este caso entre SS3 y SS4, para que el transformador de la SS3 contiguo pueda alimentar a la zona prevista para el transformador caído. También se podría alimentar con el transformador de la propia subestación, cerrando en este caso el seccionamiento de la propia subestación

- Situación degradada doble: Una subestación está fuera de servicio. Este caso es mucho más complicado de gestionar por parte del sistema eléctrico, ya que debe alimentar el tráfico con una subestación menos. El procedimiento en muchos casos suele ser degradar la operación en un porcentaje determinado por la Operadora del Sistema Ferroviario.

- Sistema en corriente continua: Normalmente no se suele aplicar ninguna modificación al sistema eléctrico.

- Sistema en corriente alterna: Al haber separación de fases, se deberá cerrar una zona neutra para que se pueda alimentar del adyacente más inmediato. Un ejemplo se puede ver en la Figura con la SS2.

Las situaciones degradadas, tanto por la posible degradación de la operación como por la afectación a subestaciones adyacentes, se analizan con otros ciclos de demanda. La finalidad es comprobar que la merma producida en la 
subestación, se analice correctamente teniendo en cuenta que otras subestaciones pueden estar siendo más exigidas:

- Se calcula el sumatorio de PotDemanda para la operación en situación degradada por causa de fallo en el sistema eléctrico. No se puede aplicar la misma demanda de los trenes para situación normal de funcionamiento, por lo que el sistema de optimización hará una distinción cuando esté analizando las situaciones degradadas, y trabajará solo con los ciclos calculados pertenecientes precisamente a la situación degradada.

- Se calculan el sumatorio de PotDemanda para cada subestación, o cada grupo transformador en caso de alterna. De modo que se pueda analizar el impacto sobre las subestaciones directamente afectadas por algún fallo en el sistema eléctrico. Esto permite mucha flexibilidad en los tests, ya que cuando se compruebe el estado del sistema en caso de fallo de una subestación, además de las situaciones anteriormente citadas de comprobación, se efectuarán tests para ver si el sistema es capaz de soportar el ciclo de demanda máxima para esta subestación, así como para las subestaciones más afectadas, que suelen ser las adyacentes.

La adaptación de un método de optimización a las múltiples variables que se conjugan en un sistema ferroviario y los múltiples cálculos que se deben realizar para cada escenario, provoca que se haya buscado un elemento común para examinar el dimensionamiento. La alternativa que se ha planteado este apartado es realizar el estudio previo a la ejecución del sistema de optimización, de modo que se pueda contrastar cualquier diseño que se proponga con esas condiciones calculadas inicialmente. Esto se cumple con el método que calcula las potencias máximas demandadas por los trenes en circulación durante el plan de operación diseñado.

Una de las más importantes metas de este trabajo tiene una fuerte relación con la automatización. Permite mejorar mucho los tiempos de cálculo y análisis de los sistemas eléctricos, y la aplicación directa a proyectos reales. 


\subsection{Función objetivo general}

La evaluación de un diseño de SET exige la combinación de muchas variables en un sistema de ecuaciones complejo. La complejidad del sistema ya se ha comentado en los apartados anteriores, y también la fuerte no-linealidad del mismo, el objetivo final deriva en poder modelar la evaluación del sistema con rigor matemático y accesible a modelos de optimización.

Examinando los conceptos que están integrados en el cómputo de la validez de un diseño del dimensionamiento, se llega a la conclusión que existen variables que son antagónicas en su valoración. Es decir, que aumentando el valor de una de ellas, es directamente proporcional a la disminución en valor de la otra parte del conjunto de variables involucradas en el sistema. El planteamiento para esta dualidad de alcances, debe ser la implantación de un sistema bi-objetivo. En el apartado dedicado a la explicación de las técnicas de optimización, ya se avanzó qué clases de algoritmos se iban a utilizar para alcanzar los mejores diseños, en los términos que se plantean en esta Tesis. El aspecto de confrontación entre los alcances de los dos objetivos, son la causa de la división entre las dos funciones de evaluación, es decir de las dos funciones objetivo.

Dentro de las características que marcan la naturaleza de un algoritmo de optimización multiobjetivo, está la toma de decisiones. Tanto para los algoritmos escogidos (NSGA-II, AMGA-II y ع-MOEA), como en general, para los demás, la elección del óptimo resultado, es habitual que no sea una tarea inmediata. Suele componerse de varias posibilidades, todas ellas cabrían en un conjunto de optimalidad, pero diferentes entre sí, tanto por algún valor de las variables iniciales de configuración, como del escenario final que plantean, como evidentemente también, el resultado final de las funciones objetivo. La elección final exigirá un paso más dentro del proceso. Este paso se verá apoyado por el Frente de Pareto, también llamdo de EdgeWorth-Pareto, (ver Figura 9) obtenido por el algoritmo, cuyos componentes, tienen la propiedad de nodominancia respecto al resto del conjunto de posibles soluciones.

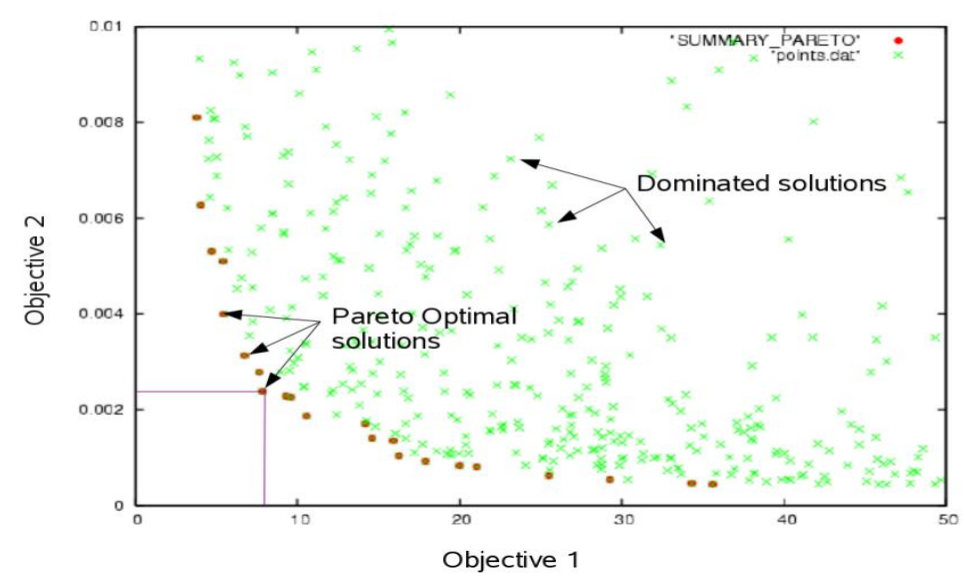

Figura 46 Ejemplo Frente Pareto (Fuente Página Web Cenaero) 
Se dice que un punto o individuo $x^{*} \in X$, si para todas las posibilidades $x \in X$ e $I=$ $\{1,2, \ldots k\}$, cuya relación:

$$
f_{1}(x)=f_{1}\left(x^{*}\right) \quad \forall x \in X y \forall i \in I
$$

donde

$\mathrm{X}$ es el espacio de soluciones

I es el número total de objetivos a optimizar

Ecuación 11. Explicación del conjunto de elementos perteneciente Frente de Pareto

Debe existir al menos alguna opción óptima, que se definiría de la siguiente forma:

$$
f_{1}(x)>f_{1}\left(x^{*}\right) \forall i \in I
$$

Ecuación 12. Explicación de dominancia de un individuo sobre otro

La condición de dominancia vendría descrita por la siguiente ecuación entre conjuntos o vectores de valores objetivo $u=\left(u_{1}, u_{2}, \ldots, u_{n}\right)$ y $v=\left(v_{1}, v_{2}, \ldots, u_{n}\right)$ si $\mathrm{y}$ solo si u es parcialmente menor a v.

Acotando la teoría de Pareto a los algoritmos multiobjetivo, el conjunto de óptimos vendría dado por la ecuación:

$$
\begin{gathered}
\mathrm{P}=\left\{\mathrm{x} \in \mathrm{X} \mid \neg \exists \mathrm{x}^{\prime} \in \mathrm{Xf}\left(\mathrm{x}^{\prime}\right) \preceq \mathrm{f}(\mathrm{x})\right\} \text {, siendo } \preceq \text { la relación de dominancia } \\
\text { Ecuación 13. Conjunto de individuos no-dominados }
\end{gathered}
$$

Finalmente, el Frente de Pareto es un conjunto P tal que:

$$
\begin{gathered}
\mathrm{FP}=\left\{\mathrm{u}=\mathrm{f}=\left(\mathrm{f}_{1}(\mathrm{x}), \mathrm{f}_{2}(\mathrm{x}), \ldots, \mathrm{f}_{\mathrm{k}}(\mathrm{x}) \mid \mathrm{x} \in \mathrm{P}\right\}\right. \\
\text { Ecuación 14. Frente de Pareto }
\end{gathered}
$$

La obtención del Frente de Pareto solo asegura la obtención de la mejor solución hallada de entre todas las evaluadas por el algoritmo que se haya implementado. No obstante, como se puede ver en la secuencia de ecuaciones hasta llegar al Frente de Pareto, se está hablando de conjuntos, no de una solución única. El concepto de Sistema Experto, conlleva que la decisión final deberá estar en manos del ingeniero o encargado de diseñar el sistema eléctrico. El conjunto final de posibles soluciones, deberá contener la información suficiente para que el ingeniero sea capaz de, en función de sus necesidades y de la configuración que plantean los diferentes componentes del conjunto, pueda elegir la que más convenga a sus intereses. Las funciones objetivo deberán contener la posibilidad de evaluar correctamente las necesidades del diseñador, de forma que los valores obtenidos se identifiquen claramente con las áreas delimitadas por cada función objetivo. Además el modelo de optimización exige la inclusión de restricciones. Estas restricciones también se evaluarán para cada posible solución, siendo una fuerte condición de no aparición en el Frente óptimo en caso de no cumplir alguna de ellas. 
Los dos objetivos planteados, los costes de Instalación y los costes de Operación, se han pensado como objetivos con valoraciones inversamente proporcionales, y por tanto cumplirían con la adaptación de las técnicas comentadas. Cuando en un proyecto ferroviario se potencia la calidad del servicio que ofrecerá la explotación, suele ser porque los costes de Instalación van a ser mayores, se demandará una inversión mayor para mejorar los sistemas de señalización, material rodante o del sistema eléctrico. Por el contrario, si disminuyen los costes de la inversión inicial, esto afectará a la operación y al mantenimiento, pudiendo incrementar de esta manera los costes asignados a esta fase.

El alcance de esta metodología siempre ha pretendido abarcar tanto los sistemas ferroviarios que se alimentan de corriente continua, como los que lo hacen con alterna. Ambos métodos tienen unas características específicas, que afectan al sistema eléctrico. De esta manera, las ecuaciones de las funciones objetivo se deberán de adecuar a cada sistema.

El sistema de ecuaciones genérico sería:

$$
\begin{gathered}
\mathrm{y}=\mathrm{f}(\mathrm{x})=\left\{\mathrm{f}_{1}(\mathrm{x}), \mathrm{f}_{2}(\mathrm{x})\right\} \\
x=\left\{x_{1}, x_{2}, \ldots, x_{l}\right\} \in X \text { (espacio de decisión) o genotipo } \\
\text { Ecuación 15. Conjunto de funciones objetivo }
\end{gathered}
$$

se ha adaptado al sistema bi-objetivo que se está tratando. La optimización por tanto, busca en este caso la minimización de los costes derivados de la evaluación de ambas funciones.

Las restricciones, cuya función principal es la de penalizar fuertemente la violabilidad de alguna de ellas, se representan de forma genérica:

$$
\left\{\begin{array}{l}
g(x)=\left\{g_{1}(x)\right\} \geq 0 \\
h(x)=\left\{h_{1}(x)\right\}=0
\end{array}\right.
$$

Ecuación 16. Restricciones genéricas algoritmo multiobjetivo

En los siguientes apartados, las funciones objetivo serán explicadas de forma genérica, dando las claves que posteriormente serán aplicadas en los dos sistemas de electrificación. Las restricciones o penalizaciones tenidas en cuenta se explican en el último subapartado.

\subsubsection{Codificación común del modelo de sistema.}

Un paso importante en la arquitectura de implantación de algoritmos genéticos es la codificación/decodificación. El tipo de dato que se va a tener en cuenta para representar alguna variable del modelo general, puede ser de distinto tipo: binario, entero, real... Dependiendo de la capacidad que tenga el algoritmo escogido para trabajar con los tipos de datos y también, dependiendo de la capacidad real de concretar en ese tipo lo que se quiere representar, deberá ser escogido para crear el Genotipo del problema [58]. 
En este capítulo, al igual que en el caso de las funciones objetivo, se van a trazar las líneas generales de trabajo. En los apartados correspondientes a cada sistema de electrificación se expondrán las características de cada uno y cómo afecta al diseño del modelo.

El tipo de dato elegido ha sido el Real. En esta primera aproximación se van a explicar la codificación/decodificación de los elementos Subestación de tracción y Catenaria. La elección de la representación en los genotipos es consecuente con la importancia elevada que tienen ambos elementos dentro de los dimensionamientos eléctricos habituales. En los procesos de decisión las claves de la calidad del mismo, vienen en gran medida expuestos por estos dos componentes. Incluso en los estudios de viabilidad, son los factores determinantes con los que se suele trabajar, incidiendo en mejoras o no, en función de los resultados que ofrecen las simulaciones o cálculos realizados para la demanda de explotación de la línea.

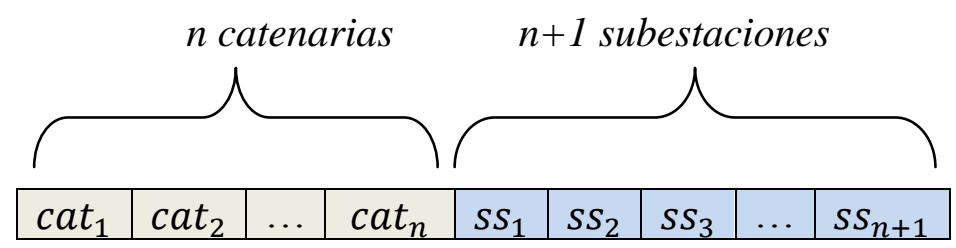

Figura 47 Estructura genotipo genérico

La distribución tiene como objeto representar las subestaciones de tracción que se instalarían en el trazado ferroviario. El número de bits representativos vendrá calculado por el número de zonas de instalación (ver apartado 4.2). Cada zona de instalación como mucho va a poder tener una subestación de tracción. El número de bits representativos de las catenarias será dependiente del número de bits de subestación. El algoritmo se ha pensado para que tenga flexibilidad para colocar un tipo de catenaria en zonas tales que:

- Entre el inicio del trazado y la primera subestación

- Entre dos subestaciones adyacentes

- Entre la última posible subestación y el final de línea

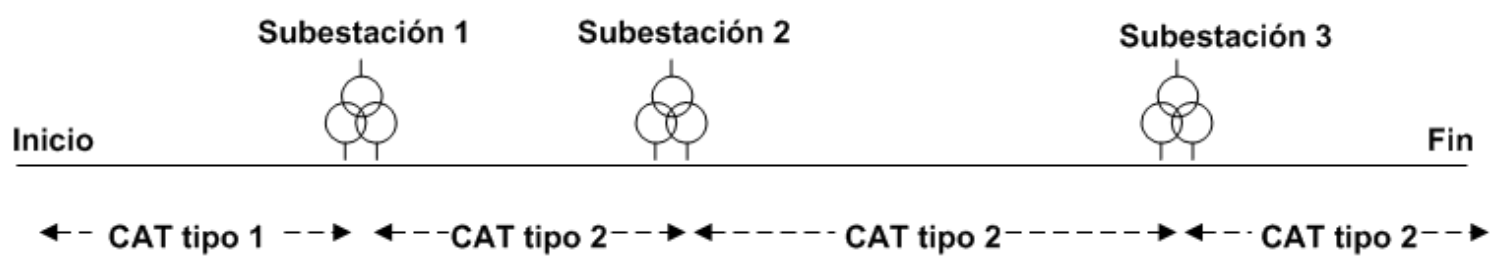

Figura 48 Ejemplo distribución catenarias

De este modo se puede testear un conjunto mayor de posibilidades de diseño y ver las consecuencias en cuanto a los costes relacionados. En la figura 11, se muestra con un ejemplo cuál es el planteamiento y la flexibilidad que aporta.

El estudio del dimensionamiento eléctrico incluye otro factor muy utilizado. Las caídas de tensión en la línea debido a un nivel de explotación elevado, o el nivel de 
sobrecarga de las subestaciones, provoca que muchos estudios de diseño modifiquen las subestaciones de tracción, de modo que se aumenten/disminuyan el número de grupos de transformación/rectificación.

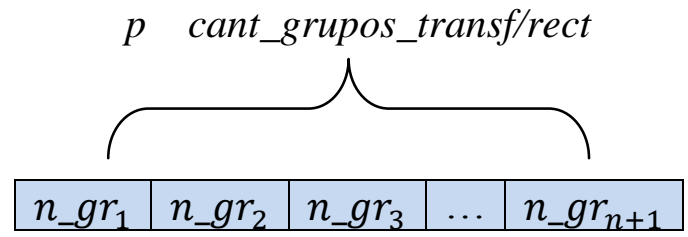

Figura 49 Ampliación genotipo a cálculo grupos en subestación

Para cada subestación que se quiera estudiar la cantidad idónea de grupos que debería tener según la optimización, deberá añadir un bit en el genotipo. Existe una flexibilidad de incluir o no el estudio de grupos de la $i$-subestación, porque en algunos proyectos puede ser que la subestación ya esté construida o bien no se tenga planeado modificar la potencia que va a generar. Hay que tener en cuenta que si bien es una habilidad del algoritmo que puede ser muy útil, el aumento del número de bits en el genotipo, también incrementa la dificultad de obtener una solución óptima en un tiempo adecuado. La idoneidad de incluir nuevos bits en función de la capacidad del algoritmo para encontrar una solución en tiempo razonable, y la mejora en la solución que puede aportar, se explicará con más detenimiento en los apartados relativos a las pruebas.

La flexibilidad y versatilidad de uso de los tipos en los genotipos que hacen uso los algoritmos escogidos, es una de las causas por las que se eligieron. Los operadores clásicos para tipos binarios, se han podido adaptar perfectamente a la dinámica de los algoritmos, y por tanto, los operadores de selección, cruce y mutación pueden trabajar adecuadamente con el tipo elegido, el Real. La elección final del tipo Real para todos los bits de los genotipos, obedeció a lo siguiente:

- Mayor versatilidad para discretizar una serie de valores

- Cubrían la necesidad de valores continuos

- Mayor facilidad para la programación e implementación en los algoritmos genéticos

\subsubsection{Codificación para sistemas en corriente alterna del modelo de sistema.}

Autotransformadores y Zonas Neutras deben ser incorporadas a la información genómica del modelo aplicado de esta Metodología. La información requerida acerca de los Autotransformadores es, además de las características eléctricas, la posición y cantidad de cada uno de ellos. De las Zonas Neutras lo más significativo siempre es la posición donde se van a situar. Al igual que se ha detallado la codificación para los elementos comunes, se va a realizar para los elementos propios de corriente alterna. 
En la Figura 13 se muestra la ampliación para el caso de Autotransformadores.

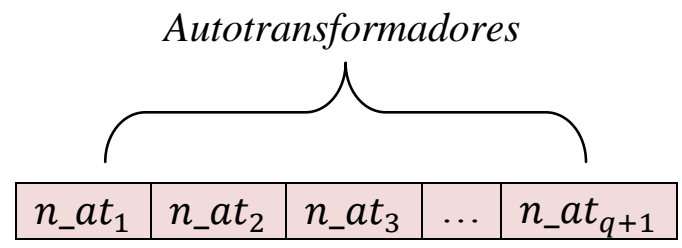

Figura 50 Ampliación genotipo a posicionamiento y número de Autotransformadores

Cada gen asociado a los Autotransformadores va a representar una zona específica de la línea donde existe la posibilidad de poder instalar uno. Está clasificado de la siguiente forma:

- Entre inicio de la línea y primera subestación de tracción

- Entre subestación y zona neutra

- Entre la última subestación instalada en la línea y el final de línea

Se identifican todas las posibles zonas del escenario que se esté tratando. Si el escenario elimina alguna subestación, entonces las zonas de instalación de autotransformadores relacionadas, no se tienen cuenta, lo que quiere decir que el gen asociado no va a ser decodificado para encontrar los autotransformadores a instalar.

Y finalmente la codificación de las Zonas Neutras se presenta en la Figura siguiente:

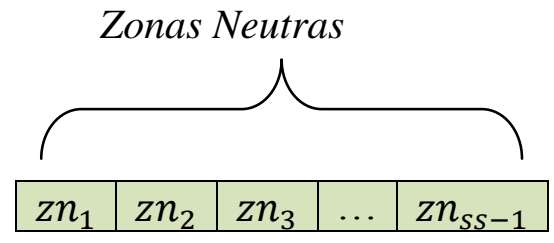

Figura 51 Ampliación genotipo a posicionamiento de Zonas Neutras

En el planteamiento para la Metodología se ha establecido en que se va a instalar una zona neutra por cada dos subestaciones de tracción adyacentes que haya en el sistema. Por tanto, Como mucho se deberán posicionar el número de subestaciones posible menos uno. Ese será precisamente el número final de genes representativos de las Zonas Neutras. Estas zonas neutras sirven para separar subestaciones, pero si las subestaciones tienen más de un grupo de transformación, por configuración se añade una zona neutra para separar las fases de ambos grupos. Esta zona neutra funciona independientemente del algoritmo genético, es implícita a la subestación, es decir, donde se sitúe la subestación, allí se instalará esta zona neutra entre transformadores.

\subsubsection{Codificación/Decodificación Catenaria}

Cada bit de catenaria debe ser capaz de proponer un tipo de sección transversal a la zona que le es asignada. El número real que represente a cada zona de catenaria se va a resolver de la siguiente manera.

- $\quad$ El número real pertenece al ámbito: $R \in[0,1]$ 
- Cada zona de catenaria pertenecerá a la estratificación formada por la división $\frac{1}{n^{\circ} \text { catenarias }}$, donde la cantidad de zonas de catenaria viene determinada por el criterio explicado en la Figura 11.

- El valor real representativo de cada bit buscará secuencialmente a qué zona pertenece la catenaria, es decir si hubieran 3 posibles catenarias, y el genotipo para ese bit tuviera un valor de 0.5 la catenaria para la zona ocupada por ese bit sería la segunda $\left(0.333+0.333>0.5 \rightarrow 2^{\mathrm{a}}\right.$ catenaria $)$.

\subsubsection{Codificación/Decodificación Subestación de tracción. Grupos de transformación/rectificación de potencia.}

Las subestaciones de tracción ha sido el elemento principal a partir del cual pivotan los demás elementos en nuestro planteamiento. En el apartado de discretización zonal se ha mencionado, entre otras, un tipo de zona, Instalación. Este tipo de zona se va a asociar a la posibilidad de instalar una subestación en ella. La forma ideada para distribuir estas zonas es la siguiente:

- Según dimensionamiento o cálculo inicial de número de subestaciones posibles a instalar, se divide proporcionalmente la longitud del trazado entre ese número.

- El valor obtenido será la longitud perteneciente a cada zona de instalación, y consecuentemente, la cantidad de zonas de instalación será igual al número de subestaciones posibles a instalar.

Para asegurar la instalación de una subestación en cualquier punto de la línea, era necesario un tipo de dato que cubriera todo el espectro. Para ello era necesario un tipo real $R \in[0,1]$. En este caso, el valor que contuviera el bit en cuestión, sería el proporcional a la posición dentro del área de instalación correspondiente. Esa posición será fácilmente decodificable y el módulo eléctrico del simulador sería capaz de modificar la posición de la subestación.

Pero era necesario un paso más: la posibilidad de eliminar del dimensionamiento cualquier subestación. Para ello se pensó que el ámbito del bit debería cambiar a $R \in[$ ValorNegativo, 1]. Siendo el ValorNegativo, la barrera entre 0 y ese valor, que equivaldría en caso de estar en esa zona el valor del bit, la eliminación de esa subestación para el dimensionamiento representado por el genotipo. En este caso hay que considerar que si ese valor es muy grande respecto al ámbito general, la probabilidad de que el valor sea negativo aumentan, y en la generación de nuevos individuos para las generaciones siguientes, aumentaría mucho la posibilidad de eliminar subestaciones, aspecto que dependiendo de la necesidad de instalar la subestación, puede que no sea el mejor camino para llegar al óptimo. Esta serie de casuísticas se explicarán detalladamente en capítulos posteriores.

\subsubsection{Codificación/Decodificación Autotransformadores.}

Tanto los Autotransformadores como las Zonas neutras, como ya se ha explicado, tienen una fuerte conexión con las Subestaciones de tracción. De modo que es muy 
importante examinar el estado de cada escenario y contar el número de subestaciones que siguen presentes en la instalación del dimensionamiento propuesto por cada genotipo.

El primer paso de la decodificación debe ser identificar todas las posibles zonas del escenario que se esté tratando. Si el escenario elimina alguna subestación, entonces las zonas de instalación de autotransformadores relacionadas, no se tienen cuenta, el gen no se evaluaría.

Para el posicionamiento de los autotransformadores, se incluye un nuevo parámetro en la configuración, cuya función será establecer la distancia mínima entre los Autotransformadores y la Subestación de tracción en su área de influencia, de modo que dictamina un margen de posicionamiento, que será finalmente el calculado por el valor que contenga el gen. El tipo de dato escogido es $R \in[0,1]$.

El siguiente esquema se especifican los pasos a seguir

$$
\begin{aligned}
& \text { pos }_{\text {at }}=\text { LímiteFinal } \\
& \text { mientras PosicAT > LimiteInicial } \\
& \text { crear NuevoAT } \\
& \text { NuevaPosicAT }=(\text { SeparaciónEntreSS }+(\text { ValorGen } * \text { Márgen })) \\
& \text { PosicAT }=\text { NuevaPosicAT } \\
& \text { Diagrama } 1 \text { Explicación búsqueda posición y cantidad de Autotransformadores }
\end{aligned}
$$

Donde

LimiteInicial es la posición de la anterior Subestación o inicio de línea

LímiteFinal es la siguiente Subestación o final de línea

Márgen es la distancia mayor a la que se pueden separar los autotransformadores adyacentes

Este algoritmo permite poder calcular el número y posición dependiendo del valor del gen, de la parametrización inicial y de la posición entre inicio y final de zona de instalación de los autotransformadores. Con esta información, el programa es capaz de modificar el diseño eléctrico para situar los autotransformadores resultantes.

\subsubsection{Codificación/Decodificación Zonas Neutras.}

El tipo de dato usado es de nuevo el real $R \in[0,1]$. Para obtener si una zona neutra se va a instalar solo hay que comprobar que las subestaciones de tracción adyacentes están en funcionamiento, es decir, que el genotipo las ha incluido en su diseño. Si es así, para entrar la posición exacta de situación, la decodifiación se basará en el valor proporcional marcado por el gen respecto de la longitud total entre las dos 
subestaciones. El propósito siempre es que sea posible analizar cualquier posibilidad, y siguiendo este procedimiento, se consigue.

\subsubsection{Función objetivo de costes de Instalación}

El primer paso para definir la función objetivo de los costes de instalación fue conseguir el conjunto de componentes que debían involucrarse en este tipo de costes. Por tanto este conjunto está conformado por aquellos elementos que según los proyectos realizados, demanda habitual y experiencia propia, pedían una atención especial a la hora de crear el diseño de los sistemas de energía. Los componentes elegidos dependen del tipo de sistema eléctrico con el que se esté trabajando.

\subsubsection{Función objetivo para sistema de electrificación en corriente continua}

- Costes Subestación de tracción

○ Grupos de transformación/rectificación y potencia que suministran

- Tipo de catenaria

- Dependiendo de la calidad de los materiales que componen los cables principales, el tipo de carril usado, si se hace uso o es necesario algún feeder de refuerzo o para el retorno, todo esto hace que el coste difiera de un tipo de catenaria a otro. Además la instalación de la catenaria y posterior mantenimiento también influye en los costes, por lo que debe ser analizado precisamente.

- Zona de instalación

- La zona donde se van a instalar los elementos propios del dimensionamiento, pueden contener complejidades que se deben tener en cuenta para la evaluación del coste final. La función objetivo deberá tener en cuenta este factor e incluir su evaluación.

- Zonas medioambiental

- Al igual que para zona de instalación, la función objetivo deberá tener en cuenta la inclusión de instalación en alguna zona de ponderado valor ecológico o de intrusión sobre el medio ambiente.

- Zona de conexión a la red general

- La dificultad medida en la ponderación de este tipo de zonas, deberá ser tenida en cuenta en el cálculo de costes de instalación.

La decodificación del genotipo representado, conlleva que se pueda calcular su fenotipo asociado. La función objetivo genérica para costes de instalación es:

$$
\begin{gathered}
\mathrm{f}_{1}(\mathrm{x})=\sum_{\mathrm{k}=1}^{\mathrm{nsubst}}\left(\operatorname{CostCG}_{\mathrm{k}}+\operatorname{CostCE}_{\mathrm{k}}+\operatorname{CostSs}_{\mathrm{k}}\right)+\operatorname{CostOhl} \\
\text { Ecuación 17. Función Objetivo } 1 \rightarrow \text { costes de Instalación }
\end{gathered}
$$


Donde $\operatorname{CostSs}_{\mathrm{k}}$ son los costes ponderados de la k-ésima subestación de tracción y CostOhl es el coste de la catenaria instalada en cada zona.

$$
\begin{aligned}
& \qquad \operatorname{CostCG}_{\mathrm{k}}=\delta_{\mathrm{k}} *\left(\frac{\mathrm{zi}_{\mathrm{k}}-\mathrm{zi}_{\mathrm{k}}}{\mathrm{zinst}}\right)+\vartheta_{\mathrm{k}} *\left(\frac{\mathrm{zge}_{\mathrm{k}}-\mathrm{zgi}_{\mathrm{k}}}{\mathrm{zgen}}\right) \\
& \text { Ecuación 18. Costes de conexión con la red general de distribución }
\end{aligned}
$$

Donde

CostCG $\mathrm{k}_{\mathrm{k}}$ coste asociado a la instalación de la subestación k en una zona de conexión con la red general eléctrica

$\delta$ coste de la zona de instalación

$\vartheta$ coste de la zona de conexión a la red general eléctrica

$\mathrm{zie}_{\mathrm{k}}$ posición final de la subestación k dentro de la zona de instalación [m]

zii $_{\mathrm{k}}$ posición inicial de la subestación k dentro de la zona de instalación [m]

zinst longitud total de la zona de instalación [m]

zgen longitud total de la zona de conexión a la red general eléctrica [m]

zge $_{\mathrm{k}}$ posición final de la subestación $\mathrm{k}$ dentro de la zona de conexión a la red general $[\mathrm{m}]$

$\mathrm{zgi}_{\mathrm{k}}$ posición final de la subestación $\mathrm{k}$ dentro de la zona de conexión a la red general $[\mathrm{m}]$

Representan los costes derivados de la dificultad de la conexión con las acometidas de la red general de distribución

$$
\operatorname{CostCE} E_{\mathrm{k}}=\delta_{\mathrm{k}} *\left(\frac{\mathrm{zi}_{\mathrm{k}}-\mathrm{zii}_{\mathrm{k}}}{\mathrm{zinst}}\right)+\gamma_{\mathrm{k}} *\left(\frac{\mathrm{zee}_{\mathrm{k}}-\mathrm{zei}_{\mathrm{k}}}{\mathrm{zenv}}\right)
$$

Ecuación 19. Costes de instalación en zona medioambiental de impacto

$\operatorname{CostCE}_{\mathrm{k}}$ coste asociado a la instalación de la subestación $k$ en la zona con afectación medioambiental

$\gamma$ coste asignado a la zona con afectación medioambiental

zee $_{\mathrm{k}}$ posición final de la subestación k dentro de la zona medioambiental [m]

zei $_{\mathrm{k}}$ posición inicial de la subestación k dentro de la zona medioambiental [m]

zenv longitud total de la zona medioambiental [m]

Representan los costes derivados del impacto sobre el medioambiente 


\subsubsection{Función objetivo para sistema de electrificación en corriente alterna}

El sistema de electrificación en alterna puede constar de otros elementos distintos a los incluidos para el sistema en continua:

- Costes Autotransformadores

- Zona Neutra

La función objetivo debe tener en cuenta estos dos elementos, así que la ecuación queda:

$$
\begin{gathered}
\mathrm{f}_{1}(\mathrm{x})=\sum_{\mathrm{k}=1}^{\mathrm{nsubst}}\left(\operatorname{CostCG}_{\mathrm{k}}+\operatorname{CostCE}_{\mathrm{k}}+\operatorname{CostSs}_{\mathrm{k}}\right)+\operatorname{CostOh}+\sum_{\mathrm{l}=1}^{\mathrm{nAT}} \operatorname{CostAT}_{\mathrm{l}}+ \\
\sum_{\mathrm{m}=1}^{n Z n} \operatorname{CostZn}_{\mathrm{m}}
\end{gathered}
$$

Ecuación 20. Función Objetivo $1 \rightarrow$ costes de Instalación (AC)

Donde CostAT y $\operatorname{CostZn}_{\mathrm{m}}$ son los costes respectivos de los Autotransformadores y de las Zonas Neutras que tenga el diseño del genotipo $x$.

\subsubsection{Función objetivo de costes de Operación}

Los costes de operación se han considerado como aquellos que aparecen durante la explotación del sistema. La relevancia de los mismos debe estar ligada al tipo de dimensionamiento diseñado, y también deben representar una medida adecuada para analizar el impacto del mismo.

- Zona de mantenimiento

- Dependiendo de la posición ocupada por los elementos instalados para el sistema eléctrico, las tareas de mantenimiento pueden tener una complejidad mayor o menor. La Ponderación calculada para cada zona se tendrá que aplicar en la evaluación de estos costes.

- Pérdidas de energía a lo largo de la línea

- La distribución de la energía para la tracción parte de las subestaciones de tracción hacia los trenes circulando en la línea. La impedancia en los cables de la catenaria provocan pérdidas a lo largo de la línea. Se trata de calcular el balance de pérdidas según el valor obtenido de potencia aparente generada por las subestaciones menos la potencia eléctrica demandada por los trenes. Este valor calculado proporciona un índice de calidad del dimensionamiento en el instante de tiempo estudiado.

$$
\text { Perdidas }=\sum_{\mathrm{r}=1}^{\text {nsubst }} \text { Ssubst }_{\mathrm{r}}-\sum_{\mathrm{u}=1}^{\text {trenes }} \text { Pet }_{\mathrm{u}}
$$


Donde

nsubst es el número de subestaciones de tracción instaladas

trenes es el número total de trenes circulando por la línea en el ciclo de demanda de potencia estudiado

Pet $_{u}$ es la Potencia eléctrica demandada por cada tren. Se aplica el factor de potencia para calcular la potencia aparente [VA]

Ssubst $_{\mathrm{r}}$ es la potencia calculada proveniente de cada subestación de tracción [VA]

Finalmente la función objetivo:

$$
f_{2}(x)=\sum_{v=1}^{\text {nsubst }}\left(\operatorname{CostCM}_{v}\right)+\alpha * \text { Perdidas }
$$

Ecuación 22. Función objetivo 2, que se calcula los costes de Operación

$\operatorname{CostCM}_{\mathrm{v}}$ es el coste relacionado con el mantenimiento

$\alpha$ es el factor de conversión a valores de coste, se divide por VA.

$$
\operatorname{CostCM}_{\mathrm{v}}=\delta_{\mathrm{v}} *\left(\frac{\mathrm{zie}_{\mathrm{v}}-\mathrm{zii}_{\mathrm{v}}}{\text { zinst }}\right)+\rho_{\mathrm{v}} *\left(\frac{\mathrm{zme}_{\mathrm{v}}-\mathrm{zmi}_{\mathrm{v}}}{\mathrm{zmaint}}\right)
$$

Ecuación 23. Calculo de los costes de zonas de mantenimiento

Donde

$\delta_{\mathrm{v}}$ es el coste de zona de instalación

$\rho_{\mathrm{v}}$ es el coste de zona de mantenimiento

$\mathrm{zme}_{\mathrm{v}}$ posición final de la subestación k dentro de la zona de mantenimiento [m]

$\mathrm{zmi}_{\mathrm{v}}$ posición inicial de la subestación k dentro de la zona de mantenimiento [m]

zmaint longitud total de la zona de mantenimiento [m]

\subsubsection{Restricciones}

Las restricciones tienen relación con aspectos fundamentales de la viabilidad del proyecto, de modo que si alguna de ellas no se cumple, afecta muy negativamente al genotipo representado, penalizando mucho su inclusión en generaciones venideras en el árbol de búsqueda de los algoritmos genéticos

\subsubsection{Convergencia de la simulación eléctrica}

En la descripción de la herramienta Hamlet, en el Capítulo II, se explica cuál es el criterio de convergencia del cálculo eléctrico. En caso que el cálculo eléctrico del 
sistema representado por el genotipo resuelva que el escenario no es convergente, se aplicará una penalización muy alta, de modo que sea muy complicado el traspaso de ese tipo de configuración en las siguientes generaciones.

$$
h(x)=\operatorname{conv}(x)=\left\{\begin{array}{c}
1, \text { si no converge } \\
0, \text { si converge }
\end{array}\right\}
$$

Ecuación 24. Restricción que comprueba la convergencia del dimensionamiento eléctrico

\subsection{Sistema de electrificación de corriente continua}

Las pruebas de convergencia desarrolladas en corriente continua tienen muy en cuenta el comportamiento del sistema en situaciones degradadas:

- Estudio de situaciones de degradadas simple

Se simula con la subestación con un grupo menos. Para adecuar el sistema a la nueva situación, se calcula la impedancia de pérdidas de la subestación, teniendo en cuenta la caída del grupo.

$$
Z=\frac{\text { ResistInterna }}{100} * U_{n}^{2} / S_{i}
$$

Ecuación 25. Fórmula aplicada al cálculo de la impedancia de pérdidas de la subestación de tracción

Donde

ResistInterna es el valor porcentual de coeficiente resistivo de la subestación

$U_{n}^{2}$ es la tensión nominal al cuadrado

$S_{i}$ es la potencia de la subestación

- Estudio de situaciones de degradada doble

Se simula el escenario con la subestación fuera de servicio y con el ciclo de demanda de potencia mayor para la situación degradada de la operación del sistema ferroviario. A continuación si el escenario es Convergente, se comprueba si para el ciclo de potencia de más demanda para la subestación caída, también es Convergente.

- Estudio de tensiones admisibles en catenaria

Para todas las situaciones testeadas, en caso de que se haya pasado el Test de Convergencia, se examinarán las tensiones máximas y mínimas en catenaria, y las tensiones de retorno. En caso de sobrepasar los límites, tendrá una penalización en la restricción.

\subsection{Sistema de electrificación de corriente alterna}

Las pruebas de convergencia desarrolladas en corriente continua incluyen: 
- Estudio de situaciones de degradadas simple

Se simula con la subestación con un grupo menos. En este caso, se tiene que cerrar la zona neutra contigua y observar el comportamiento del sistema. Para los tests, se van a buscar las zonas neutras adyacentes para que la tracción pueda ser alimentada correctamente en el sector dañado. A continuación se busca el ciclo de carga máxima para cada subestación que esté suministrando potencia a la que ha sufrido el percance y se comprueba la Convergencia del escenario.

- Estudio de situaciones de degradada doble

Se simula con el escenario con la subestación fuera de servicio y con el ciclo de demanda de potencia mayor para la situación degradada de la operación del sistema ferroviario. Como se puede alimentar de las dos subestaciones adyacentes (en caso que esté en esa situación), se examinan ambas posibilidades, conjuntamente con los ciclos de máxima demanda para cada una de las utilizadas.

- Estudio de tensiones admisibles en catenaria

Para todas las situaciones testeadas, en caso de que se haya pasado el Test de Convergencia, se examinarán las tensiones máximas y mínimas en catenaria, y las tensiones de retorno. En caso de sobrepasar los límites, tendrá una penalización en la restricción.

\subsubsection{Desbordamiento del presupuesto}

La otra restricción importante que se ha considerado es la limitación de ejecución de la instalación completa del sistema eléctrico. La limitación se calcularía en función de una cantidad límite presupuestaria que no debería ser rebasada en ningún caso. Si la suma de costes de las dos funciones objetivo sobrepasa esa cantidad, previamente estimada por el usuario según sus necesidades, se aplicaría una penalización elevada al genotipo o individuo, dificultando el paso a generaciones futuras del mismo o de sus genes.

$$
\operatorname{TotalCSc}(\mathrm{x})=\mathrm{f}_{1}(\mathrm{x})+\sum_{\mathrm{p}=1}^{\text {nsubst }} \operatorname{CostCM}_{\mathrm{p}}
$$

Ecuación 26. Cálculo de los costes ponderados-monetarios asociados al dimensionamiento representado por el genotipo $\mathrm{x}$

$$
\mathrm{g}(\mathrm{x})=\operatorname{DesvPresup}(\mathrm{x})=|\operatorname{ValConf}-\operatorname{TotalCSc}(\mathrm{x})|
$$

Ecuación 27. Restricción que evalúa el límite presupuestario del dimensionamiento eléctrico representado por el genotipo $\mathrm{x}$

Donde ValConf es el valor estimado por el usuario representativo del límite presupuestario asumible. 


\subsection{Conclusiones}

A lo largo del Capítulo se han descrito los diferentes módulos con los que cuenta la Metodología para realizar las evaluaciones oportunas y que finalmente se pueda dar al ingeniero/diseñador un conjunto de posibles soluciones. Se ha descrito el proceso de búsqueda de los algoritmos de optimización que finalmente se ha implementado, dando las causas y las características básicas de los mismos. Se ha señalado cómo se han integrado tanto los elementos principales que actúan en un sistema eléctrico, conjuntamente con la zonificación de ciertos baremos críticos a tener en cuenta en un proyecto de esta envergadura. Finalmente se ha desarrollado tanto las funciones objetivo, como el sistema de codificación/decodificación que usarán los algoritmos genéticos, y no menos importante el llamado punto de focalización de un sistema eléctrico, personificado en los picos de demanda máxima de potencia, calculados en simulaciones previas. 


\section{Bibliografía}

[1] L. T. Biegler and I. E. Grossmann, "Retrospective on optimization," Computers \& chemical Engineering, vol. 28(8), pp. 1169-1192, 2004.

[2] I. E. Grossmann and L. T. Biegler, "Part II. Future perspective on optimization," Computers \& Chemical Engineering, vol. 8, no. 28, pp. 1193-1218, 2004.

[3] G. B. Dantzig, "Linear programming and extensions," Princeton University Press, 1963.

[4] S. P. Hans, "Superlinearly convergent variable metric algorithm for general nonlinear programming problems," Mathematical Programming, vol. 1, no. 11, pp. 263-282, 1976.

[5] M. Miyatake and K. Matsuda, "Optimal speed and charge/discharge control of a train with onboard energy storage devices for minimum energy operation," Electrical Drives, Automation and Motion in Power Electronics. IEEE International Symposium on SPEEDAM 2008, pp. 1211-1216, 2008.

[6] M. Fesanghary, M. Mahdavi, M. Minary-Jollandan and Y. Alizadeh, "Hybridizing harmony search algorithm with sequential quadratic programming for engineering optimization problms," Computer methods in applied mechanics and engineering, vol. 33, no. 197, pp. 3080-3091, 2008.

[7] Z. W. Geem, J. H. Kim and G. V. Loganathan, "A new heuristic optimization algorithm: harmony search," Simulation, vol. 76, no. 2, pp. 60-68, 2001.

[8] G. L. Nemhauser and L. A. Wolsey, Integer and combinatorial optimization, New York: Wiley/Interscience, 1988.

[9] C. Barnhart, E. L. Johnson, G. L. Nemhauser, M. W. Savelsbergh and P. H. Vance, "Branchand-price: Column Generation for solving huge integer programs," Operations Research, no. 46, pp. 316-329, 1998.

[10] E. Balas, S. Ceria and G. Cornuejobs, "A lift-and-project cutting plane algorithm for mixed 0-1 programs," Mathematical Programming, vol. 58, pp. 295-324, 1993.

[11] M. A. Duran and I. E. Grossman, "An outer-approximation algorithm for a class of mixedinteger nonlinear programs," Mathematical Programming, vol. 36, p. 307, 1986.

[12] J. F. Benders, "Partitioning procedures for solving mixed variables programming problems," Numerical Mathematics, vol. 4, pp. 238-252, 1962.

[13] T. Westerlund and F. Petterson, "A cutting plane method for solving convex MINLP problems," Computers and Chemical Engineering, vol. 19, pp. S131-S136, 1995. 
[14] E. Leyffer, Generalized Outer Approximation, US: Springer, 2009.

[15] G. R. Kocis and I. E. Grossmann, "Relaxation strategy for the structural optimization of process flowsheets," Industrial Engineering in Chemical Research, vol. 26, no. 1869, 1987.

[16] G. S. GmbH, "GAMS," 2015. [Online]. Available: http://www.gams.com/.

[17] D. o. C. Engineering, "MINOPT," Princeton University, 1998. [Online]. Available: http://titan.princeton.edu/MINOPT/.

[18] V. Pontryagin, V. Boltyanski, R. Gamkrelidge and E. Mishchenko, The mathematical theory of optimal processes, New York: NY:Interscience, 1962.

[19] B. Bojko and R. Luus, "Use of random admissible values for control in iterative dynamic programming.," Industrial Engineering in Chemical Research, vol. 31, pp. 1308-1314, 1992.

[20] I. Fonseca and G. Leoni, Modern Methods in the Calculus of Variations: L^ p Spaces, Springer Science \& Business Media, 2007.

[21] H. G. Bock and K. Plitt, "A multiple shooting algorithm for direct solution of optimal control problems," in In Proceedings of the 9th IFAC world congress, Budapest, 1984.

[22] J. T. Betts, "Practical methods for optimal control using nonlinearprogramming," in Advances in design and control. SIAM, Philadelphia, 2001.

[23] Al-Khayyal and A. Faiz, "A. Generalized bilinear programming: Part I. Models, applications and linear programming relaxation," European Journal of Operational Reserach, vol. 60, no. 3, pp. 306-314, 1992.

[24] K. Boese, A. Kahng and S. Muddu, "A new adaptive multi-start technique for combinatorial global optimizations," Operations Research Letters, vol. 16, no. 2, pp. 101-113, 1994.

[25] H. Schwandt and E. Hansen, "Global Optimization using internal analysis," ZOR, vol. 42, pp. 250-250, 1995.

[26] P. Hansen, B. Jaumard and S. Lu, "Global optimization of univariate Lipschitz functions: II. New algorithms and computational comparison," Mathematical Programming, vol. 55, no. 1-3, pp. 273-292, 1992.

[27] J. A. Nelder and R. Mead, "A simplex method for function minimization," The Computer Journal, vol. 7, no. 4, pp. 308-313, 1965.

[28] M. A. Muñoz, J. A. López and E. F. Caicedo, "Inteligencia de enjambres: sociedades para la solución de problemas (una revisión)," Ingeniería e investigación, no. 28, pp. 119-130, 2008. 
[29] F. Glover, "Tabu search Part I," ORSA Journal of Computing, vol. 1, no. 13, pp. 190-206, 1989.

[30] M. Mitchell, An introduction to Genetic algorithms, MIT press, 1988.

[31] T. Back, D. Fogel and Z. Michalewicz, Handbook of evolutionary computation, IOP Publishing Ltd., 1997.

[32] L. Davis, Genetic algorithms and simulated annealing, 1987.

[33] J. Kennedy, "Particle swarm optimization," Enclyclopedia of Machine Learning, pp. 760766, 2010.

[34] J. Holland, Adaptation in natural and artificial systems, 1992.

[35] V. Pareto, Cours D'Economie Politique, Vols. I-II, Lausanne: F. Rouge, 1896.

[36] D. Van Veldhuizen and G. Lamont, "Evolutionary computation and convergence to a pareto front.," in En Late breaking papers at the genetic programming , University of Wisconsin, Madison, 1992.

[37] C. A. Coello, "A comprehensive survey of evolutionary-base multiobjective optimization techniques," Knowledge and Information systems, vol. 1, no. 3, pp. 269-308, 1999.

[38] K. Deb, A. Pratap, S. Agarwal and T. A. Meyarivan, "A fast and elitist multiobjective genetic algorithm: NSGA-II.," IEEE Transactions on Evolutionary Computation, vol. 6, no. 2, pp. 182-197, 2002.

[39] J. Hartmanis and R. E. Stearns, "On the computational complexity of algorithms," Transactions of the American Mathematical Society, pp. 285-306, 1965.

[40] K. Deb, S. Agrawal, A. Pratap and T. Myarivan, "A fast elitist non-dominated sorting genetic algorithm for multiobjective optimization: NSGA," Lecture notes in computer science, no. 1917, pp. 849-858, 2000.

[41] K. Deb, M. Mohan and S. Mishra, "Towards a quick computation of well-spread paretooptimal solution," Evolutionary multi-criterion optimization, pp. 222-236, 2003.

[42] S. Tiwari, G. Fadel and K. Deb, "AMGA2: improving the performance of the archive-based micro-genetic algorithm for multi-objective optimization," Engineering Optimization, vol. 4, no. 43, pp. 377-401, 2011.

[43] S. Tiwari, P. Koch, G. Fadel and K. Deb, "AMGA: An Archive-based Micro Genetic Algorithm for," in GECCO, Atlanta, Georgia, USA, 2008.

[44] R. Storn and K. Price, "Differential Evolution - A Fast and Efficient Heuristic for Global Optimization over continuos spaces," Journal of global optimization, vol. 11, pp. 341-359, 
1997.

[45] W. L. Loh, "On the asymptotic distribution of some randomized quadrature rules," in Stein's Method and Application, C. Stein, AD Barbour and LHY Chen, 2005, pp. 209-222.

[46] M. Domínguez, A. Fernández-Cordador, A. Cucala, T. Gonlsaves and A. Fernández, "Mutiobjective particle swarm optimization algorithm for the design of efficient ATO speed profiles in metro lines," Engineerign applicatins of artificial intelligence, vol. 29, pp. 43-53, 2014.

[47] R. Eberhart and Y. Shi, "Comparison between Genetic algorithms and Particle Swarm optimization," Evolutionary programming VII. Lecture notes in Computer Science, vol. 1447, pp. 611-616, 2005.

[48] E. Ziztler and L. Thiele, "Multiobjective evolutionary algorithms: a comparative case study and the strength Pareto approach," Evolutionary Computation, vol. 3, no. 4, pp. 257-271, 1999.

[49] E. Elbeltagi, T. Hegazy and D. Grierson, "Comparison among five evolutionary-based optimization algorithms," Advanced Engineering Informatics, vol. 19, no. 1, pp. 43-53, 2005.

[50] T. Suzuki, "DC power-supply system with inverting substations for traction systems using regenerative brakes," IEE Proceedings B (Electric Power Applications), vol. 129, no. 1, 1982.

[51] C. T. A. 203, "Aplicaciones Ferroviarias. Tensiones de alimentación de las redes de tracción. UNE-EN 50163," AENOR, Madrid, 2005.

[52] R. J. Hill, "Electric Railway Traction. Part 1: Electric traction and DC traction motor drives," Power Engineering, pp. 47-56, 1994.

[53] M. Tang, L. Qun-zhan and H. Jian-min, "Structure of Hybrid Power filter for reactive power and harmonic currents in traction," Power system technology, vol. 2, no. 11, 2004.

[54] C. t. A. 203, "Aplicaciones de ferrocarriles - Instalaciones fijas. Parte 1: Medidas de protección relacionadas con la seguridad eléctrica y puesta a tierra," AENOR, Madrid, 1999.

[55] C. T. A. 203, "Aplicaciones ferroviarias. Instalaciones fijas. Transformadores de tracción. UNE-EN 50329," AENOR, Madrid, 2004.

[56] E. Pilo, Diseño óptimo de la electrificación de ferrocarriles de alta velocidad. Tesis Doctoral., Madrid: Unviersidad Pontifica Comillas, 2003.

[57] D. González and F. Manzanedo, "Optimal Design of a D.C. Railway Power Supply System," 
in Electric Power , Canada, 2008.

[58] J. H. Holland and D. E. Goldberg, "Genetic Algorithms and machine learning," Machine Learning, vol. 3, no. 2, pp. 95-99, 1988. 


\section{Aplicación de la Metodología en Líneas Ferroviarias de DC. Pruebas y Resultados.}

El enfoque de trabajo siempre ha estado dividido en comprobar primero la eficiencia de la metodología en sistemas que funcionen con corriente continua (DC), y posteriormente adaptar e investigar cómo debería cambiar para optimizar sistemas con corriente alterna (AC). Los dos artículos publicados (ver anexo Publicaciones y Ponencias) reflejan esta división. Por esta razón se van a detallar los sistemas en apartados distintos.

Este primer apartado de Aplicación está dedicado a los sistemas ferroviarios que tienen sistemas de electrificación en corriente continua. Tras una explicación del sistema en general, estado del arte y adaptación al sistema experto, se explicarán las características principales de las líneas escogidas para las pruebas y un breve análisis del dimensionamiento de cada configuración inicial. Finalmente se mostrarán y detallarán el proceso de optimización, los resultados finales dependiendo de parámetros y algoritmo genético usado y por supuesto, un breve análisis del dimensionamiento resultante para cada caso.

\subsection{Introducción y Estado del Arte.}

Tras una breve exposición de las características básicas de los sistemas eléctricos de tracción en los sistemas ferroviarios en corriente continua, se detallará los resultados realizados acerca del estado del arte en cuanto a optimización del diseño y, en definitiva, de las características de los sistemas de energía que se están tratando en este capítulo.

\subsubsection{Sistemas de corriente continua}

La adaptación de la metodología al sistema de corriente continua ha sido inmediata debido a que lo básico, presentado en el Capítulo 2, se corresponde con este sistema de electrificación. Cabe decir que si se han introducido novedades a lo largo de los trabajos e investigaciones, y que se presentan en los distintos casos de trabajo que se exponen en los capítulos de pruebas y ensayos de Casos.

\subsubsection{Estado del arte}

El diseño y el trabajo de optimización de un sistema de energía de tracción se basa habitualmente, en la experiencia acumulada a lo largo de los proyectos realizados y en las pautas marcadas por la normativa y regulación vigente. Tanto para los sistemas en corriente alterna como en corriente continua, los pasos a seguir son los que se muestran en la Figura 5 del Capítulo 4, con la salvedad de que podía incluso, no estar involucrado ningún software de ayuda. Con esta metodología aplicada es evidente que muchas decisiones no podían estar dentro de los parámetros de fiabilidad por la sencilla razón de la multivariabilidad de la que ya se ha comentado, que tiene como característica este tipo de sistemas. Por este motivo, además de los simuladores de análisis ferroviario, han 
ido apareciendo diversas líneas de investigación para mejorar esta toma de decisiones. En este capítulo se van a explicar las más relevantes que tienen en cuenta el sistema de electrificación en corriente continua.

En la Tesis realizada por W.D Johnston [1] se puntualiza el interés que puede llegar a tener el estudio de la optimización del sistema de energía ferroviario, y aunque en la época cuando este trabajo fue desarrollado los sistemas de electrificación aplicados al ferrocarril aún no eran tan dominantes como pueden serlo en la actualidad, el autor ya consideró la planificación para la obtención de los costes de implementar y construir el diseño eléctrico, y consecuentemente, cómo encontrar la vía de investigación o la serie de cálculos que puedan calibrar certeramente el ratio de coste-calidad del diseño determinado.

El salto de calidad se produce cuando se introducen métodos que aprovechando los estudios que reflejan cuánto de buena es una infraestructura ferroviaria según qué criterio, además son capaces de obtener una metodología que haga mejorar esos diseños. En los siguientes trabajos se especifican las técnicas usadas de optimización además del impacto sobre la capacidad de tomar decisiones que aportan los mismos. Obviamente, la progresión de la capacidad de computación ha incidido claramente en los algoritmos y herramientas de simulación, y por tanto la inclusión y adaptación de las capacidades de computación han ido de la mano con los avances en las técnicas formales de optimización

No todas las técnicas para mejorar el dimensionamiento utilizan sistemas o algoritmos de optimización. En [2] el autor se limita a modelar adecuadamente el sistema ferroviario y en concreto aquellos que trabajan con el sistema de electrificación DC a 1500 V. Conjugando el modelado con dos simuladores, uno para la operación ferroviaria y otro para la del sistema de energía, es capaz según el número de inversores de la energía proveniente del frenado, poder ir acotando por medio del sistema de lanzamiento de diversas pruebas, el número y posición más adecuadas de las subestaciones de tracción.

Hay diferentes trabajos orientados a la optimización de la red eléctrica ferroviaria, más concretamente en la tarea de mejorar los costes de inversión y la fiabilidad del sistema final construido. Relacionado con esto, en [3] los autores trabajan con un estudio analítico, para mejorar el sistema de aporte de energía ferroviario. Para alcanzar este propósito, los autores hacen uso de un simulador (Railsim), con el cual consiguen los datos y resultados necesarios para poder llegar a conclusiones acerca del dimensionamiento eléctrico del sistema ferroviario de Zagreb. Este método, según un algoritmo iterativo de análisis de flujos de carga/prueba, llega a la conclusión que las condiciones del sistema eléctrico son muy mejorables, y el objetivo que se marcan los autores, es disminuir lo máximo posible las corrientes circulantes. Para ello, uno de los factores determinantes es trabajar con las secciones y material de los cables conductores. El trabajo está centrado en sistemas de electrificación de corriente continua. 
Encontrar el método que proporcione una infraestructura ferroviaria más equilibrada, es desde luego una tarea muy interesante desde un punto de vista económico, y por esta razón los autores [4], tratan de obtener mejoras que redunden en ese objetivo final. En este caso tratan de minimizar las pérdidas de energía en sistema ferroviario DC. Para ello modelan la dinámica que afecta a los trenes usando un sistema de ecuaciones. También adecúan su modelo a las pendientes por las que circula el tren, y también el sistema de tracción del tren. Por otra parte, modelan el flujo de carga de las subestaciones y por último modelan el efecto de la subestación en una posición dada y en el plan de operación del sistema ferroviario. Al resolver el modelo hallan resultados eléctricos que les sirve para determinar si la posición de las subestaciones han proporcionado valores dentro de la normativa vigente (tensión corriente,...). De esta manera van buscando posicionamientos nuevos, que permitan una vez cumplidos los requisitos normativos, disminuir las pérdidas de energía.

Uno de los aspectos que se han destacado a lo largo de esta Tesis, es la gran variabilidad y casuística de este tipo de sistemas. La mayoría de autores que se acercan a intentar mejorar la capacidad del diseño de este tipo de sistemas, tratan de integrar en el proceso de optimización, una herramienta que evalúe la mayor cantidad de casos posible y que tenga en cuenta la mayor parte de las variables críticas. En [5] se hace uso de una técnica de optimización de Inteligencia Artificial, los algoritmos Inmune [6]. El uso del algoritmo de optimización con el objetivo de minimizar los costes generales que se producen en el consumo de energía de los inversores de potencia y los transportes de raíles llamados MRT, cuyas siglas en inglés significan Massive Rapid Transit. En concreto este sistema (Taipei MRT) trabaja con una tensión nominal de $750 \mathrm{Vcc}$, y trata de buscar el mejor posicionamiento para los inversores de potencia, que están integrados en el modelo de subestación de tracción, de modo que la potencia inyectada en el tercer carril no provoque ninguna distorsión al plan de explotación. Los autores consideran la demanda total de los trenes, formulan los costes del consumo de energía y tienen en cuenta en una ponderación calculada la confiabilidad del sistema.

Otro trabajo realizado sobre sistemas DC e integrando algoritmos genéticos se encuentra en [7]. Los autores se centran en este caso en hallar la mejor posición para las subestaciones de tracción. Para la optimización hacen uso directamente del paquete Galib [8], librería implementada en C++.

Los sistemas de transporte llamados PRT (Personal Rapid Transit), están siendo adaptados en entornos donde las distancias no son muy grandes, los movimientos de personas no son muy elevados y tampoco la magnitud de la obra civil para la infraestructura se asemeja a la de los otros sistemas ferroviarios. Algunos de los más avanzados se encuentran en Aeropuertos, y sirven sobre todo para comunicar terminales. Una de las diferencias con los MRT, es que la magnitud es mucho mayor que en el caso de los PRT, es decir las exigencias de demanda de energía están en otra escala. El auge de este tipo de sistemas ha llevado a distintos trabajos de optimización del sistema de energía. En el trabajo [9] los autores han desarrollado un proceso de optimización, en el cual los principales objetivos son conocer la posición y número de 
subestaciones o Power Supplies (PS), como es llamado en el artículo, y minimizar las pérdidas de energía. El método para realizar esto es creando un modelo de malla eléctrica donde están situados los PRT's, y los PS a lo largo de la línea; con un concepto que lo han denominado aislamiento virtual de los $P S$, y por último un proceso iterativo de cálculo.

El denominador común de los trabajos de mejora de la optimización de los sistemas de energía para la tracción, suele ser el posicionamiento de las subestaciones de tracción. Otro de los ejemplos se encuentra en [10], que de nuevo se centra en los MRT como sistema ferroviario de estudio. Para la optimización integran el algoritmo de optimización basado en búsqueda tabú [11]. Además de la elección de este sistema de optimización y cómo lo adecúa al modelo del problema que se está tratando, otro de los focos de atención se centra en la optimización del consumo de energía gracias al análisis de la influencia de los ángulos de disparo de los rectificadores de potencia de las subestaciones, y el efecto que provocan en el consumo global del sistema. Otra referencia al estudio anterior, la encontramos en el trabajo [12], en cuanto a trabajo con sistemas MRT. En este caso la técnica de optimización usada pertenece a algoritmo genéticos, es la llamada bi-criterio de optimización. Los autores realizan un esquema de cómo se simulan los sistemas eléctricos típicos, para a continuación significar los parámetros básicos de un sistema DC. Lo interesante es el modelo que gestionan para modelar los instantes en que las subestaciones usan el rectificador de potencia, en fase de demanda de tracción, o usan el inversor, en fase de frenado regenerativo. Por esta dualidad de objetivos enfrentados hace falta el algoritmo bi-criterio, y de este modo los autores asignan un peso dentro de la función objetivo que marca la intención de buscar mejor opción por una de las dos posibles, y dependiendo de los ángulos de disparo que se van generando estocásticamente por el devenir del algoritmo, se entra en la búsqueda del óptimo dual.

Existen caminos de investigación mucho más enfocados a sistemas analíticos. Uno de los más significativos se puede revisar en [13]. Esta línea de trabajo incide directamente en los flujos de carga que acontecen en el sistema, y cómo afectan al rendimiento global. De este modo pueden conocer las necesidades puntuales y poder mejorar la capacidad del mismo. Otro método analítico es el realizado por el autor [14], que en este caso estaba muy dirigido a sistemas $\mathrm{AC}$, pero que también es aplicable a sistemas DC, por esta razón se explicará también en el Capítulo 5, la parte más extensa de este trabajo. La parte común del trabajo, y que se puede aplicar a sistemas de corriente continua, es aquella en la cual el autor realiza un barrido de todas las posibilidades de alimentación desde la subestación a los trenes que están circulando en sectores próximos a las mismas. A partir de un modelo analítico de posibilidades, le permite conocer según la situación derivada del sistema ferroviario que se esté estudiando, que espacio de separación de las subestaciones es la más conveniente.

Conocer la planificación de la posible expansión que se deba hacer a futuro en una línea ferroviaria, relacionándola con las subestaciones de tracción es lo que los autores tratan de conseguir [15]. Los autores como aspecto principal, modelan el 
comportamiento de los convertidores de las subestaciones y calculan la demanda de potencia anual que va a necesitar la línea para su plan de explotación. Para la función objetivo tienen en cuenta los costes de inversión y las caídas de tensión provocada por los trenes, y para optimizar aplican la técnica de Programación dinámica. Con esto tratan de disminuir los costes de una posible expansión del dimensionamiento eléctrico.

Existen otro tipo de trabajos que están más enfocados a mejorar aspectos concretos del dimensionamiento eléctrico. La corrosión que afecta a los raíles, y que suele tener como primera medida para evitarlos, la colocación de tramos equipotenciales entre los raíles de la vía, es uno de los problemas que los diseñadores del dimensionamiento deben tener muy en cuenta. Como el autor [16] busca un método de Control de modo que la tensión de las subestaciones de tracción no altere el sistema de toma de tierra, y por tanto la corrosión sea menor. Para conseguir este nivel de control hace uso de simuladores que le permiten conocer el estado del dimensionamiento.

La variante indicada por el último trabajo mostrado gira en torno a un control del dimensionamiento eléctrico ferroviario, que si bien es necesario e interesante para el diseñador del mismo, implica tener un mayor número de casos a tener en cuenta. Es interesante sobre todo la Metodología de Control que aplica, pero para el problema global que se está resolviendo en este trabajo requiere métodos que puedan abarcar mucho más, sabiendo de la fuerte complejidad de este tipo de sistemas.

En definitiva las aproximaciones al problema que se han relatado tienen aspectos concordantes con nuestra visión de encarar el problema, pero se ha querido adaptar y globalizar la metodología habitual, de modo que los diseñadores tengan además de la posibilidad de análisis con simuladores, que estos sean flexibles para añadir muchas funcionalidades de los sistemas, un algoritmo genético potente, con un modelo de función objetivo que contenga y potencie posible diversidad del dimensionamiento, y la aplicabilidad de costes colaterales de la construcción y operación del sistema, así como que evalúe las pérdidas y la fiabilidad del mismo. 


\subsection{Líneas ferroviarias en DC para optimizar. Configuración inicial y Resultados.}

El control del proceso de optimización de un sistema eléctrico ferroviario, no solo se circunscribe a las variables que intervienen en la función objetivo. Por una parte, cualquier variación de parámetros significativos en el esquema de la línea, cambio de alzado, o algún parámetro del material rodante que modifique en gran medida la potencia a demandar, hará que esto mismo, la potencia sea distinta, o que la posición de los trenes cuando exijan más a las subestaciones se modifique, de modo que el resultado del estudio sería completamente distinto al inicial planificado. Por otra parte variaciones en la configuración de los parámetros relacionados con los algoritmos genéticos utilizados, también harán que el resultado final sea distinto.

El primero de los ejemplos de posibles modificaciones en proyectos ferroviarios descritos es más grave, ya que no depende del diseñador del proyecto. Conforme se analizan resultados de simulaciones, se añaden nuevas circunstancias o demandas de explotación a la infraestructura, se limita el presupuesto, se tienen en cuenta en estadio inicial una gama de posibles trenes a implantar, esto hace que se deban realizar ajustes en el dimensionamiento y por tanto, tener que volver a lanzar simulaciones y optimizar. Las colaboraciones directas de Citef con empresas del sector ferroviario han permitido obtener y estudiar de primera mano proyectos ferroviarios reales. Desde un primer momento se planificó trabajar con proyectos reales consolidados, de modo que la posible inestabilidad de la situación final estuviera más controlada, y además añade interés, debido a que los datos obtenidos fueran reales ofrecen seguridad sobre contrastar los resultados con la necesidad inicial del proyecto de dimensionamiento a optimizar.

En el segundo ejemplo de aproximación a la problemática, cambia bastante el enfoque. El usuario debe conocer el funcionamiento del algoritmo y sobre todo del impacto de cada parámetro de configuración. En el Capítulo 3, se describió la capacidad del algoritmo para dirigir la optimización en función de los pesos que se le asignen a cada variable introducida en el sistema. Esta influencia de los pesos asociados a las variables será un punto en el que nos detendremos a lo largo de la explicación de los resultados de las pruebas. Además de la variabilidad del resultado en función de las ponderaciones, está la que es provocada por los cambios en las variables propias de los algoritmos genéticos. Esta parte es muy interesante ya que permite comprobar la influencia de los cambios en los resultados finales. Estos cambios se van a producir tanto a nivel de hallar el Frente de Pareto final, como en los tiempos de cómputo general del proceso de optimización. Además al tener tres algoritmos genéticos distintos, la flexibilidad permitirá chequear la idoneidad de alguno sobre los otros dos en algún tipo de problema en concreto, o por ejemplo, llegar a conclusiones acerca del tiempo de computación versus tamaño del problema, relacionado con las respuestas obtenidas por los algoritmos. 
Para corriente continua, como se ha comentado, se han podido adoptar datos de proyectos reales. Los experimentos y pruebas que se van a relatar a continuación, se componen de dos líneas metropolitanas, cada una con su particularidad. El primer ejemplo es de una línea de metro, que va a servir para lanzar el sistema de optimización y comprobar la eficacia del algoritmo NSGA-II. La otra línea ferroviaria, pertenece a otro sistema metropolitano, pero el dimensionamiento del proyecto real era más complejo, y por ese motivo se pensó en aplicar distintas baterías de pruebas e introducir otros parámetros de trabajo, para comprobar finalmente la fiabilidad del método.

La estructura de descripción de las pruebas va a seguir el mismo patrón:

- Detalle de los parámetros de configuración de los distintos módulos

- Resultados eléctricos más relevantes de configuración inicial

- Proceso optimización

- Resultados configuración final elegida

- Conclusiones acerca de utilización de los algoritmos e impacto de cambios de parámetros sobre el proceso. 


\subsection{CASO 1: Línea de Metro. Resultados con algoritmo genético NSGA-II}

\subsubsection{Infraestructura de la línea}

Línea de metro con 13 estaciones a lo largo de su recorrido. Limitaciones de velocidad incluidas en la configuración.

\section{Perfil de cotas}

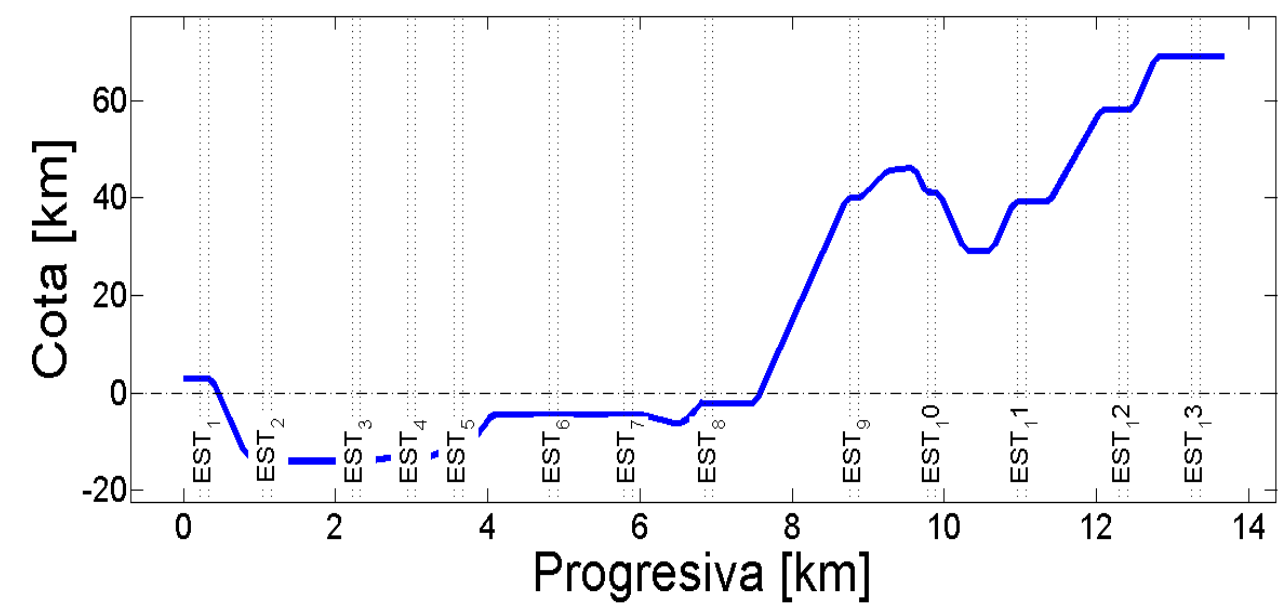

Figura 52 Perfil de alzado de línea para CASO 1

\subsubsection{Material rodante}

Configuración de la distribución de los coches del tren:
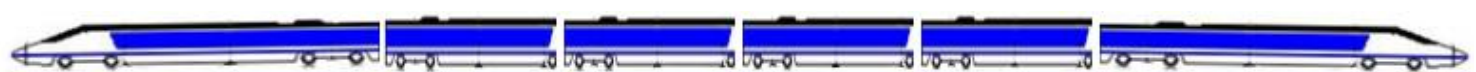

Figura 53 Ejemplo ilustrativo distribución coches en tren

M-R-R-R-R-M $\rightarrow$ Dos coches motrices y los 4 restantes remolcados

\begin{tabular}{c|c}
\hline Propiedades & Valores \\
\hline Masa & $183.579 \mathrm{Tm}$ \\
\hline Masas Giratorias & $21 \mathrm{Tm}$ \\
Pasajeros & 1204 \\
Coeficientes resistencia avance & $\mathrm{A}[N]=2892.9$ \\
Resistencia en curva (Kc) & $\mathrm{C}\left[N * \mathrm{~s}^{2} / \mathrm{m}\right]=80$ \\
Deceleración emergencia & 600 \\
Deceleración servicio & $1.3 \mathrm{~m} / \mathrm{s}^{2}$ \\
Aceleración tracción & $0.9^{2} / \mathrm{s}^{2}$ \\
\hline
\end{tabular}




\begin{tabular}{c|c}
\hline Propiedades & Valores \\
\hline Aceleración confort (jerk) & $0.6 \mathrm{~m} / \mathrm{s}^{2}$ \\
Distancia deslizamiento & $8 \mathrm{~m}$ \\
Velocidad máxima & $80 \mathrm{~km} / \mathrm{h}$ \\
\hline
\end{tabular}

Tabla 7 Propiedades material rodante CASO 1

En la Tabla 1 se han escogido los parámetros más relevantes para conseguir un comportamiento fiel del material rodante en las simulaciones. Tal y como se explicó en Capítulo 3, el procedimiento habitual incluye un conjunto de parámetros de configuración del tren, como son las curvas de tracción y frenado entre otros. Por supuesto para estas pruebas los datos eran recogidos de los datos reales del proyecto y se han introducido en el simulador.

\subsubsection{Señalización}

El sistema de señalización que se ha implantado en las simulaciones es el CBTC [17]. Usando este sistema de señalización (cantón móvil) lo que el simulador debe tener en cuenta es la posición de los trenes que le preceden, de modo que la distancia libre se calculará por la longitud entre el frontal del tren hasta la cola del que le precede. Se ha añadido por configuración una distancia de seguridad de $30 \mathrm{~m}$. Esa distancia, si no hay agujas a la contra que lo impidan, será la que usará el módulo del tren en el simulador, para el cálculo de la curva de frenado del tren, y con ello, la velocidad máxima a la que puede traccionar. Esta velocidad permitirá conocer la demanda de potencia en cada ciclo.

\subsubsection{Sistema eléctrico inicial}

Las características principales del sistema eléctrico en la fase inicial son:

- Sistema de electrificación de corriente continua DC con tensión nominal de $1500 \mathrm{~V}$

- Tipos de catenaria posibles:

\begin{tabular}{l|c|c|c|} 
& Sección $\left[\mathrm{mm}^{2}\right]$ & Resistencia $[\Omega / \mathrm{Km}]$ & Resistividad $\left[\Omega * \mathrm{~mm}^{2} / \mathrm{m}\right]$ \\
\hline $\mathrm{OHL}_{1}$ & 1080 & 0.01897 & 0.02049 \\
$\mathrm{OH} \mathrm{L}_{2}$ & 1277 & 0.01487 & 0.01899 \\
\hline
\end{tabular}

Tabla 8 Tipos de catenaria en CASO 1. Características principales 
En la configuración inicial se contemplaba la instalación de 7 subestaciones de tracción:

\begin{tabular}{|l|c|c|}
\cline { 2 - 3 } & Potencia (MW) & Resistencia interna de pérdidas $(i \Omega)$ \\
\hline Subestación & 2 grupos de 3.5 & 0.0160 \\
\hline
\end{tabular}

Tabla 9 Características básicas Subestaciones usadas en CASO 1

Escenario inicial configuración dimensionamiento eléctrico:

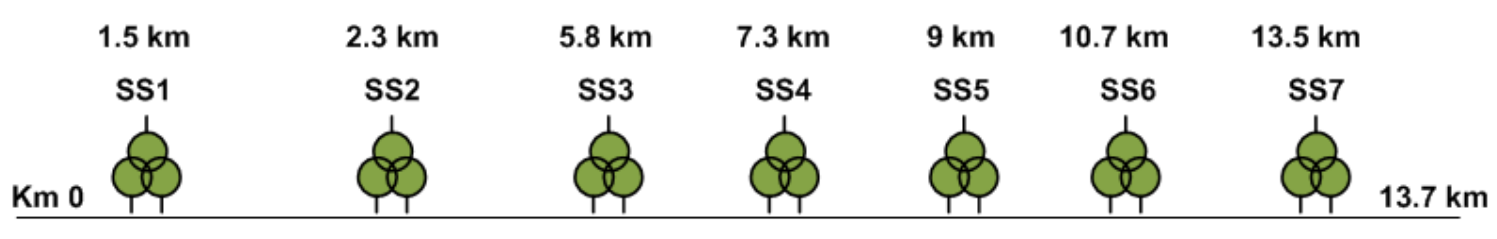

Catenaria Tipo 1

Figura 54 Configuración elementos dimensionamiento eléctrico inicial

\subsubsection{Plan de operación de la línea}

El plan de operación de la línea pretende definir el servicio que se va a ofrecer a los pasajeros. Tal y como se ha puntualizado, esta línea ferroviaria pertenece a un proyecto real de implantación de metro. Por este motivo, los datos de explotación son acordes a las necesidades de la empresa encargada de la misma:

- Los trenes deben cumplir un intervalo entre ellos de 90s

- Paradas en estación, dependiendo de la afluencia esperada de pasajeros, de entre 15 y $20 \mathrm{~s}$.

- La flota calculada es de 22-23 trenes.

\subsubsection{Configuración algoritmo genético.}

Repasando las condiciones de aplicación del algoritmo genético, y en particular de cómo se va a gestionar las variables para que puedan ser evaluadas por el optimizador (Capítulo 4), se han de planificar la discretización zonal y la ponderación que se le debe asignar a cada elemento involucrado. Es fundamental conocer la composición del genotipo a partir de los elementos básicos.

\subsubsection{Genotipo}

Según algoritmo explicado en Capítulo 4, por cada subestación de tracción será necesario un gen, y por cada tipo de catenaria otro. En total, por tanto 9:

\begin{tabular}{|l|l|l|l|l|l|l|l|l|}
\hline $\mathrm{Cat}_{1}$ & $\mathrm{Cat}_{2}$ & $S S_{1}$ & $S S_{2}$ & $S S_{3}$ & $S S_{4}$ & $S S_{5}$ & $S S_{6}$ & $S S_{7}$ \\
\hline
\end{tabular}

Figura 55 Distribución genes en Genotipo CASO 1 


\subsubsection{Configuración para la Discretización Zonal y Ponderación de elementos}

La distribución de pesos asignados se muestra a continuación.

- Ponderación de los elementos significativos:

\begin{tabular}{llllllllll}
\hline Tipo Elemento & $\mathbf{O H} L_{1}$ & $\mathbf{O H} L_{2}$ & $\boldsymbol{S S}_{1}$ & $\boldsymbol{S S}_{2}$ & $\boldsymbol{S S}_{3}$ & $\boldsymbol{S S}_{4}$ & $\boldsymbol{S S}_{5}$ & $\boldsymbol{S S}_{6}$ & $\boldsymbol{S S}_{\mathbf{7}}$ \\
\hline Peso asignado & 300 & 400 & 100 & 100 & 100 & 400 & 100 & 100 & 400 \\
\hline
\end{tabular}

Tabla 10 Pesos asignados a cada elemento

El peso de la catenaria 1 es más bajo porque analizando el material del que está formado y el precio, se ha estimado en un $25 \%$ mayor el coste de una respecto de la otra. En cuanto a las subestaciones, al ser todas iguales, el peso tan elevado de las subestación 4 y 7 respecto de las otras pretendía comprobar la funcionalidad y eficacia del algoritmo para poder eliminar esas dos, en caso que pudiera sobrar alguna y entre en litigio respecto de las adyacentes, mucho menos pesadas. 


\begin{tabular}{lcccc}
\hline $\begin{array}{l}\text { No } \\
\text { Zona }\end{array}$ & $\begin{array}{c}\text { Posición inicial } \\
(\mathbf{m})\end{array}$ & $\begin{array}{l}\text { Posición final } \\
(\mathbf{m})\end{array}$ & $\begin{array}{c}\text { Peso } \\
\text { asignado }\end{array}$ \\
\hline Zona1 & & 0 & 2000 & 135 \\
Zona2 & & 2000 & 4000 & 50 \\
Zona3 & Instalación & 4000 & 6000 & 90 \\
Zona4 & & 6000 & 8000 & 50 \\
Zona5 & & 8000 & 10000 & 135 \\
Zona6 & & 10000 & 12000 & 50 \\
Zona7 & & 12000 & 13600 & 50 \\
Zona8 & Medioambiental & 2000 & 3000 & 100 \\
Zona9 & & 9500 & 10500 & 100 \\
Zona10 & & 4000 & 4250 & 3000 \\
\hline Zona11 & & 100 & 750 & 1000 \\
Zona12 & Conexión a la red & 5000 & 7700 & 200 \\
Zona13 & general & 500 & 1700 & 250 \\
Zona14 & & 3000 & 3500 & 300 \\
Zona15 & & 8000 & 9200 & 1500 \\
Zona16 & & 0 & 1000 & 650 \\
\hline Zona17 & & 1000 & 3550 & 650 \\
Zona18 & Mantenimiento & 4200 & 4500 & 500 \\
Zona19 & & 6000 & 7700 & 200 \\
Zona20 & & 8000 & 8650 & 3350 \\
\hline
\end{tabular}

Tabla 11 Información completa acerca de la Discretización Zonal elegida

Respecto a la discretización zonal, la subdivisión de las zonas de instalación y el número de ellas se corresponde con el algoritmo diseñado. Por cada subestación equivale una zona de instalación y además todas deben tener la misma longitud.

En este proyecto no se disponía de información acerca de zonas medioambientales, ni especiales dificultades para conectar con la red general, ni tampoco valoraciones acerca de zonas críticas de mantenimiento. Los valores asignados han sido calculados y propuestos para la optimización en base a comprobar la eficacia del algoritmo, y de esta manera poder establecer el comportamiento dependiendo de los casos que se le han presentado, teniendo en cuenta esta configuración.

\subsubsection{Resultados eléctricos configuración inicial}

Este primer caso de muestra de funcionamiento de la Metodología se centra sobre todo en el análisis de las caídas de tensión en la línea.

Con el sistema completo ya configurado en el simulador Hamlet, se hizo un primer estudio aproximativo del estado del sistema eléctrico, para poder chequear qué valores presentaba y hacer una primera valoración de las posibilidades de mejora, tanto a nivel eléctrico como presupuestario o afectación a zonas críticas. En la siguiente gráfica se muestra el resultado de las Tensiones mínimas en catenaria: 


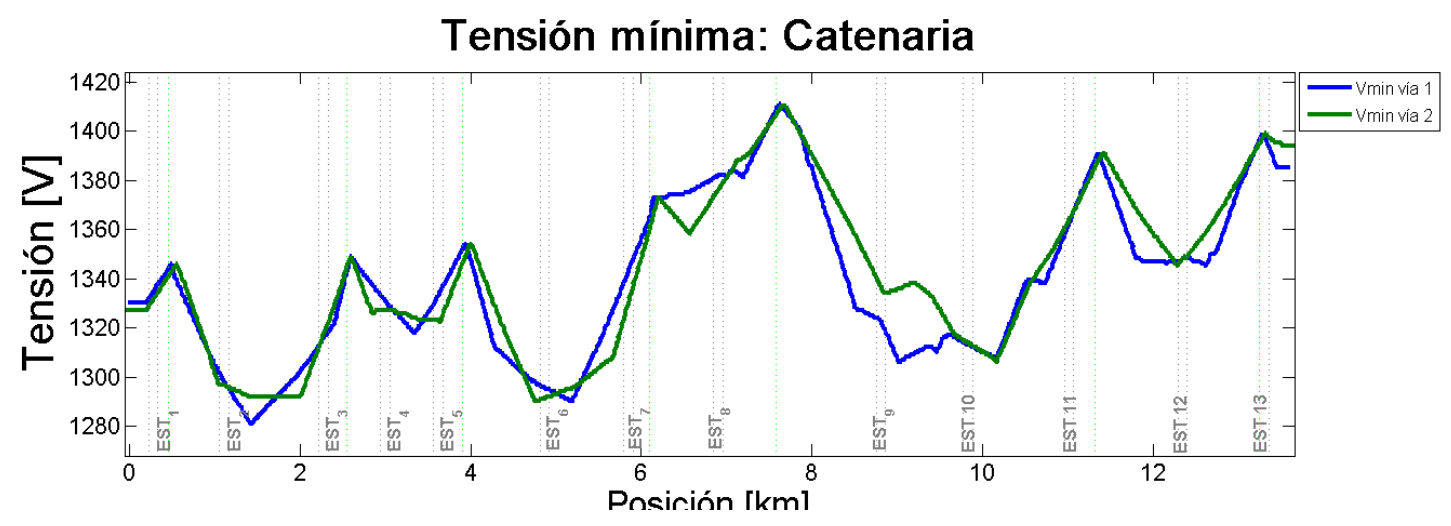

Figura 56 Tensiones mínimas en catenaria configuración inicial CASO 1

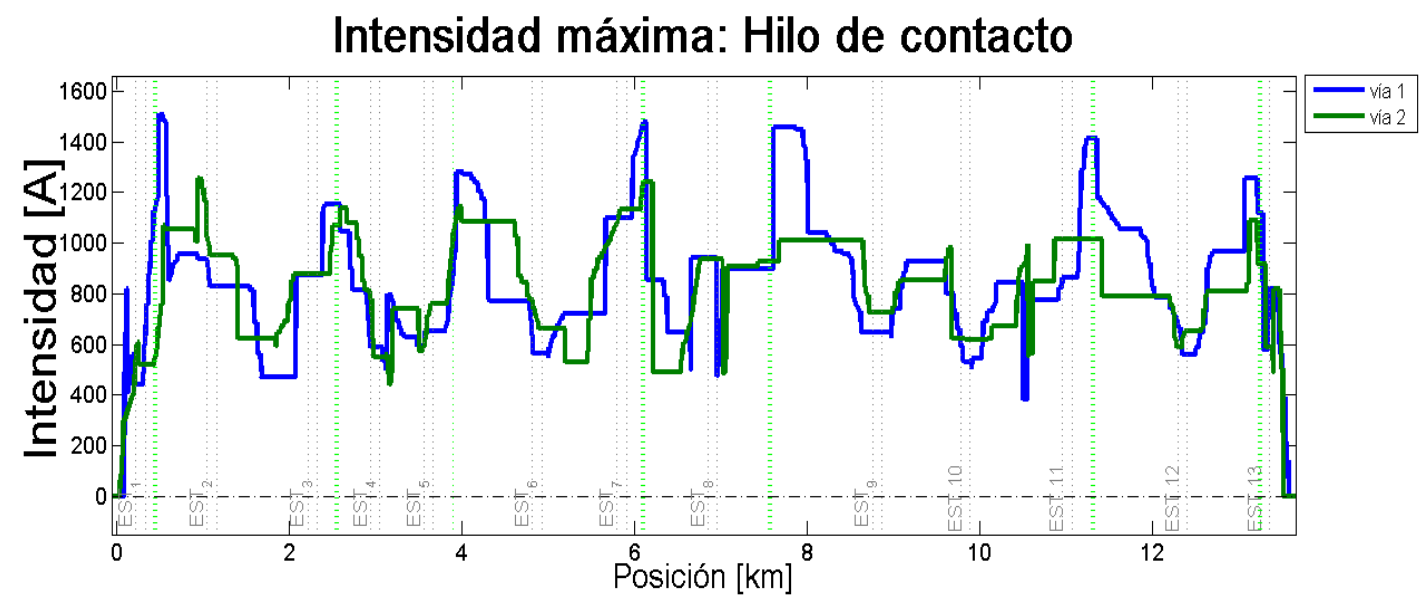

Figura 57 Intensidad máxima hilo de contacto configuración inicial CASO 1

En ambas gráficas se aprecian picos de tensión e intensidad donde están situadas las subestaciones, que permiten extraer una primera conclusión acerca del sistema eléctrico. Las tensiones mínimas en algunos puntos están próximas a la tensión nominal, y alejadas por tanto del mínimo permitido. Es posible que, aunque no se han estudiado las situaciones degradadas simples ni completas, se pueda mejorar en términos presupuestarios suprimiendo alguna subestación de tracción.

\subsubsection{Proceso optimización del CASO 1. Configuración y resultados. Análisis.}

\subsubsection{Configuración parámetros del algoritmo genético.}

Para el CASO 1 se aplicó el algoritmo genético NSGA-II. Como primera aproximación al problema, se analizó el impacto de los parámetros determinantes en la búsqueda heurística del Frente óptimo. También se pudo evaluar el impacto, y la necesidad, de incrementar el número de individuos y poblaciones para poder examinar un rango mayor de posibilidades de escenarios de dimensionamiento eléctrico final.

En la tabla 6 aparecen los valores asignados a los parámetros asociados a los operadores del algoritmo. Estos valores son los que a lo largo de las pruebas, consiguieron un mejor acercamiento a valores óptimos en el Frente de Pareto. 


\begin{tabular}{llllll}
\hline Población & № Generaciones & $\begin{array}{l}\text { Probabilidad de } \\
\text { Cruce (0-1) }\end{array}$ & $\begin{array}{l}\text { Probabilidad } \\
\text { mutación (0-1) }\end{array}$ & $\begin{array}{l}\text { Valor índice } \\
\text { distribución de } \\
\text { mutación (0- } \\
100)\end{array}$ & $\begin{array}{l}\text { Valor índice } \\
\text { distribución } \\
\text { cruce (0-100) }\end{array}$ \\
\hline $\begin{array}{l}10 / 20 \\
\text { individuos }\end{array}$ & 25 & 0.7 & 0.3 & 350 & 25 \\
\hline
\end{tabular}

Tabla 12 Valor parámetros finales NSGA-II CASO 1

La estimación de la probabilidad de cruce y mutación se pensó para incentivar la diversidad genética, ya que son valores muy altos y por tanto, permiten en gran medida la aplicación de ambos operadores.

\subsubsection{Resultados y análisis. Conclusiones.}

En el apartado anterior se ha explicado que las pruebas realizadas se pensaron para una población inicial de 10 individuos. Una de las características inherentes a los algoritmos genéticos, es que el resultado final es probable que no sea el óptimo global, sino que sea un óptimo local, y por esta razón se han de establecer una serie de pautas de conocimiento del sistema que se quiere optimizar, en relación a los parámetros del algoritmo. En este caso, para 10 individuos los Frentes de Pareto finales obtenidos no permitían asegurar que se estaba cerca del óptimo. Por este motivo, se fue incrementando hasta los 20 individuos, que en este caso, ya atendiendo a resultados con poblaciones menores, la mejora no era significante y por tanto, se analizaron en ese punto las soluciones provistas por la última generación, por su Frente de Pareto.

En este primer trabajo de optimización, no estaban integradas en las restricciones del modelo, las situaciones de convergencia de escenarios degradados, ni el análisis de tensiones extremas. En todo caso, el resultado final fue interesante desde el punto de vista de análisis de los sistemas eléctricos propuestos.

Antes de mostrar los resultados más relevantes, en las siguientes gráficas se exponen los Frentes de Pareto para la población de 10 individuos y para la de 20.

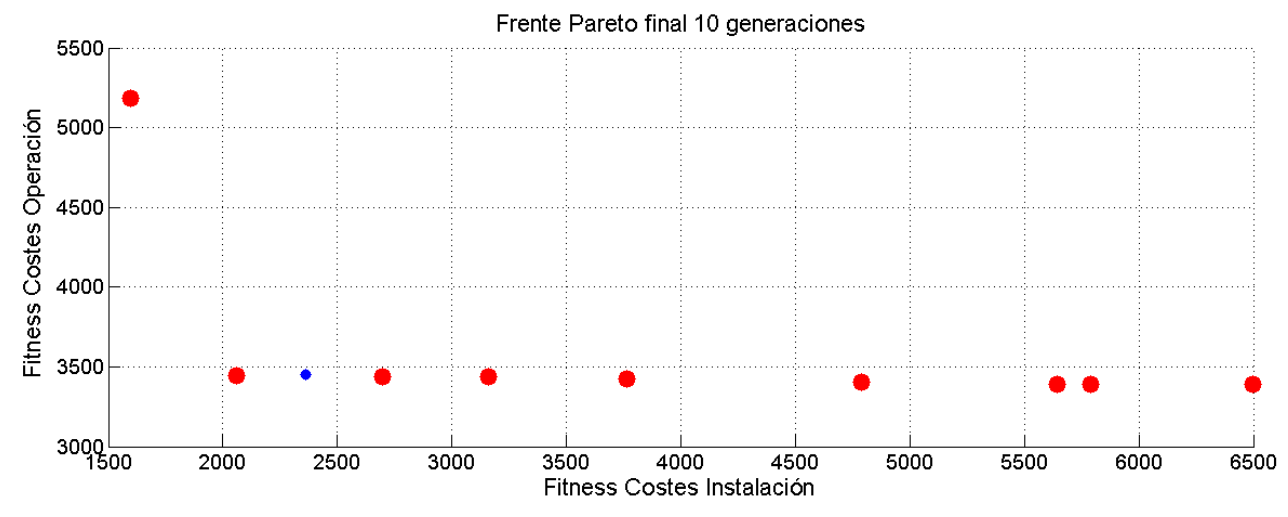

Figura 58 Frente de Pareto final para población 10 individuos CASO 1 


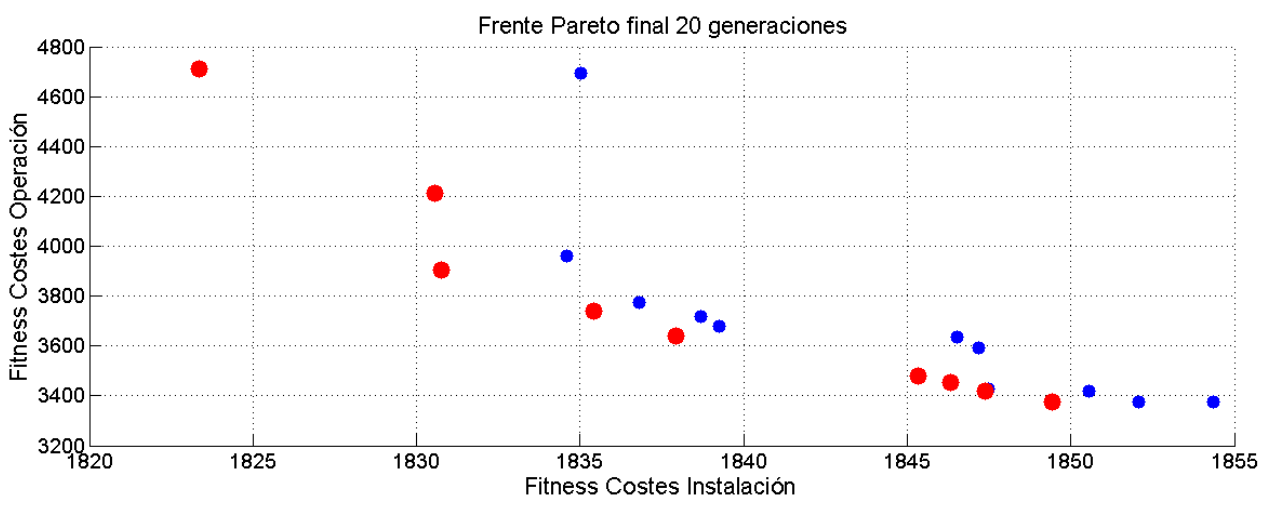

Figura 59 Frente de Pareto final para población de 20 individuos CASO 1

Se aprecia que el fenotipo para ambas funciones objetivo es sensiblemente mejor en el segundo caso. La diversidad de la población también es considerablemente mayor, lo que sin duda ayuda a explorar el espacio de soluciones posibles.

La elección final del diseño propuesto ya depende de los requisitos o interés determinado de cada diseñador o ingeniero. En este caso, la opción que se adoptó fue diseñar el sistema eléctrico con los dos genotipos que tenían el mejor valor para cada función objetivo.

\subsubsection{Diseño para mejor valor función objetivo Costes de Instalación}

La configuración propuesta por el genotipo es la siguiente:

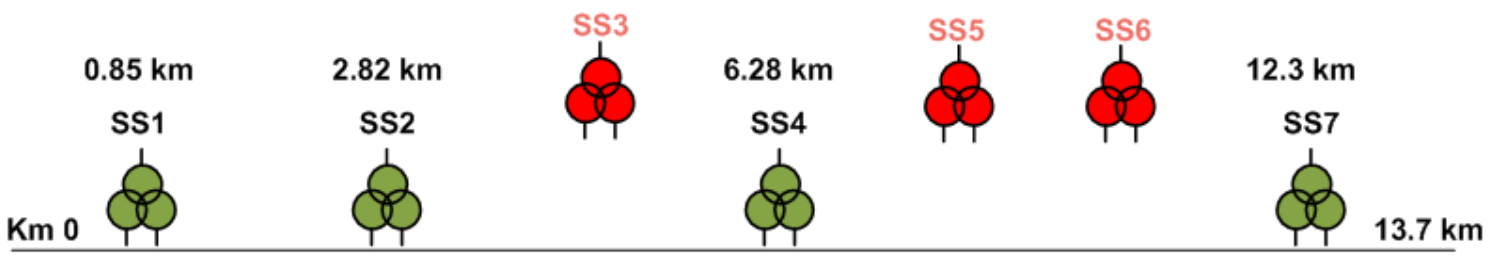

\section{Catenaria Tipo 2}

Figura 60 Configuración para mejor valor para costes de instalación CASO 1

Como se ve, se han eliminado 3 subestaciones de tracción y se ha escogido finalmente la catenaria $\mathrm{OHL}_{2}$. 


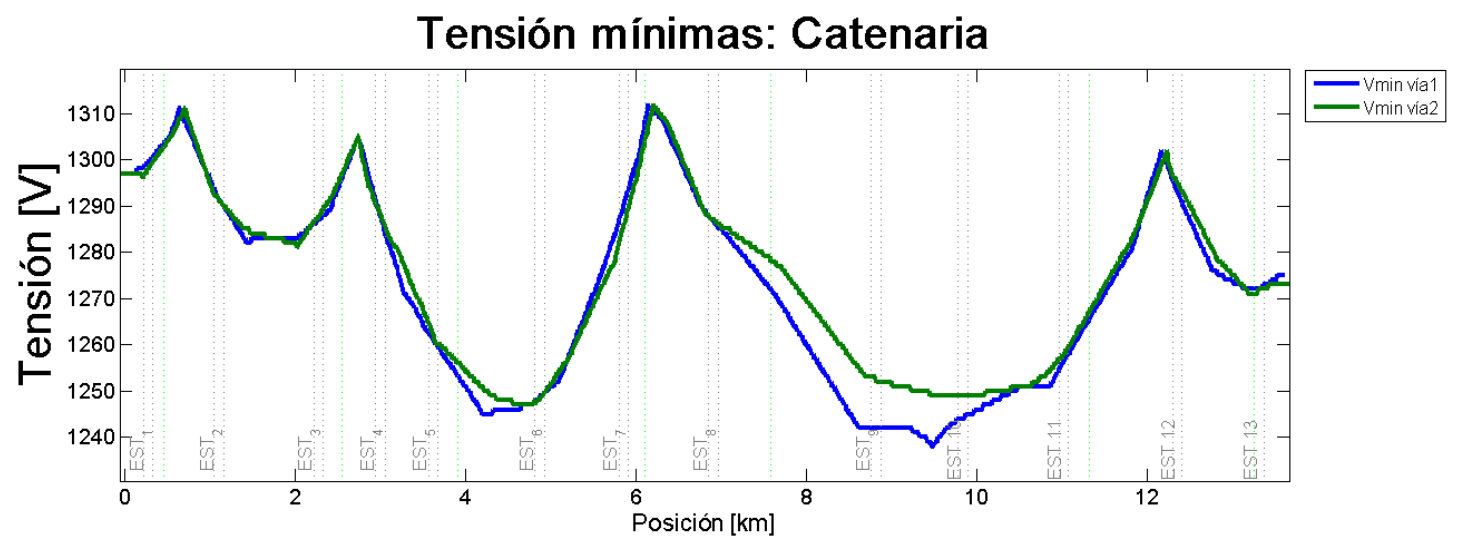

Figura 61 Tensiones mínimas para escenario con mejor valor para costes de instalación

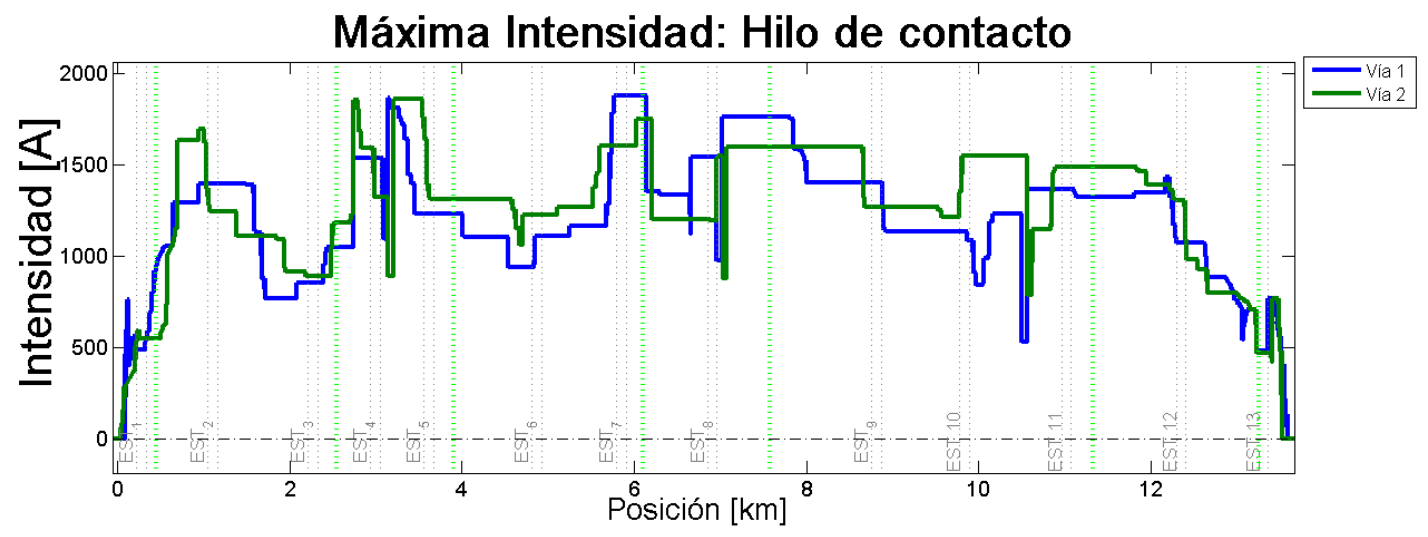

Figura 62 Intensidades máximas para escenario con mejor valor para costes de instalación

Como se observa en las gráficas, se puede ver que en este caso a pesar de estar lejos de los límites marcados por la norma, es probable que el escenario tenga problemas cuando haya alguna situación degradada, acusado este aspecto en la subestación 4. La distancia entre las subestaciones en esta solución es muy grande. En situación de degradada doble, en este caso fallo completo de subestación, la distancia a cubrir por la subestación adyacente, sería mayor. Este es uno de los escenarios que se pretende mejorar con la inclusión del análisis de situaciones de fallo en subestaciones. Esto se ha integrado en las pruebas del CASO 2, lo que permite comparar y llegar a conclusiones de viabilidad/tiempo, que serán muy útiles cuando se aplique la Metodología en casos futuros.

En cuanto al comportamiento del algoritmo respecto a evitar zonas críticas, en este caso si que se ha cumplido, ya que ninguna de las zonas con más peso asignado, ha sido propuesta para instalar en ella una subestación. 


\subsubsection{Diseño para mejor valor función objetivo Costes de Operación}

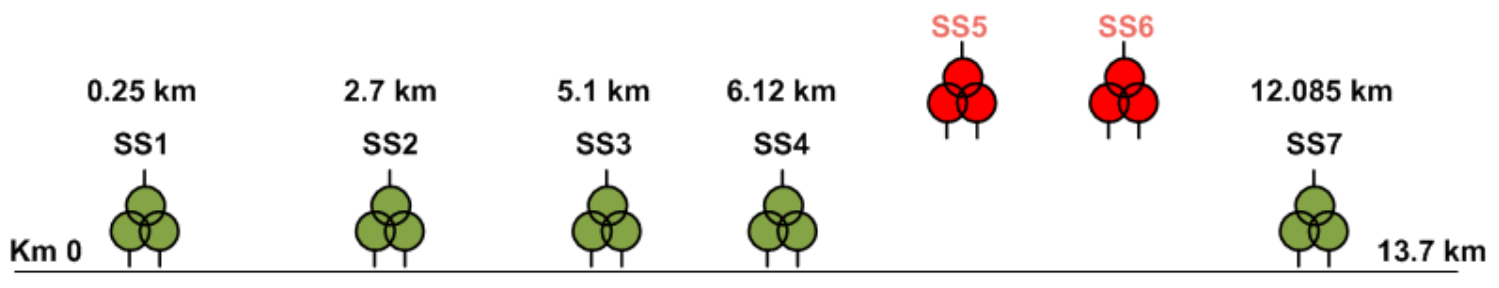

\section{Catenaria Tipo 2}

Figura 63 Configuración para mejor valor para costes de operación CASO 1

En este caso no era tan determinante el coste de los elementos, sino los costes de mantenimiento y pérdidas de energía. Por lo que el algoritmo ha tratado de evitar las zonas que más inconvenientes presentaba para el mantenimiento y preservar en la mayor medida posible las pérdidas de energía. Con todo, la solución mejor eliminaba las subestaciones 5 y 6, como se ve en la Figura 12.

\section{Tensiones mínimas: Catenaria}

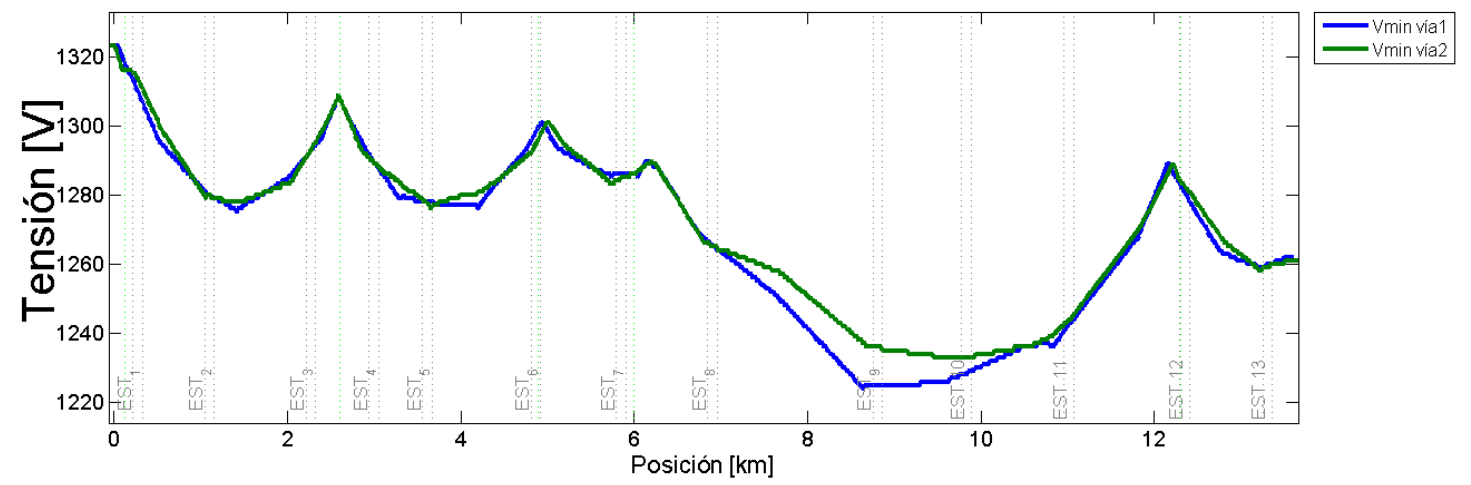

Figura 64 Tensiones mínimas catenaria para escenario con mejor valor en costes de operación CASO 1

De nuevo una de las situaciones de posible conflicto y que se debería analizar, es el fallo doble de la subestación 7. La tensión nominal para esta línea es de $1500 \mathrm{~V}$ y la distancia es muy grande entre la última subestación y el final de línea en la Estación 13. En este caso la Metodología indica situaciones de riesgo para el sistema, y dota al ingeniero de capacidad para conseguir un diseño real viable, aunque probablemente, no sea el propuesto el definitivo.

En cuanto a la zona crítica para el Mantenimiento que se sitúa alrededor del kilómetro 8-9, se hace patente que ha sido uno de los criterios básicos para que la subestación que podía estar instalada en esa zona, sea descartada. 


\subsubsection{Comparación y conclusiones}

Al no realizar análisis de situaciones degradadas, se completó el análisis con los resultados obtenidos de las Intensidades medias cuadráticas de cada escenario estudiado:

\begin{tabular}{|c|c|c|c|c|}
\hline & $\begin{array}{l}\text { Intensidad media } \\
\text { cuadrática } \\
\text { intervalos }\end{array}$ & $\begin{array}{l}\text { Escenario } \\
\text { configuración } \\
\text { inicial }\end{array}$ & $\begin{array}{l}\text { Escenario } \\
\text { mejor valor } \\
\text { Funcion } 1\end{array}$ & $\begin{array}{l}\text { Escenario } \\
\text { mejor valor } \\
\text { Función } 2\end{array}$ \\
\hline Vmin vía1 (V) & & 1281 & 1238 & 1224 \\
\hline Vmin vía 2 (V) & & 1290 & 1247 & 1233 \\
\hline I max vía 1 (A) & & 1510 & 1875 & 1890 \\
\hline I max vía 2 (A) & & 1257 & 1856 & 1686 \\
\hline \multirow{2}{*}{ SS1 } & RMC (1min) (A) & 4412.3 & 5809.7 & 5386.6 \\
\hline & $\mathrm{RMC}(30 \mathrm{~min})(\mathrm{A})$ & 3640.5 & 4819.0 & 4473.5 \\
\hline \multirow{2}{*}{ SS2 } & RMC (1min) (A) & 4186.7 & 6269.6 & 1.7 \\
\hline & RMC (30min) (A) & 3452.3 & 5184.6 & 1.4 \\
\hline \multirow{2}{*}{ SS3 } & $\mathrm{RMC}(1 \mathrm{~min})(\mathrm{A})$ & 2994.6 & \multirow{2}{*}{ No incluida } & 6240.3 \\
\hline & $\mathrm{RMC}(30 \mathrm{~min})(\mathrm{A})$ & 2445.1 & & 5044.4 \\
\hline \multirow{2}{*}{ SS4 } & RMC (1min) (A) & 2985.2 & 5845.8 & 6056.5 \\
\hline & RMC (30min) (A) & 2472.8 & 4752.8 & 5022.4 \\
\hline \multirow{2}{*}{ SS5 } & RMC (1min) (A) & 2649.9 & \multirow{2}{*}{ No incluida } & \multirow{2}{*}{ No incluida } \\
\hline & RMC (30min) (A) & 2237.0 & & \\
\hline \multirow{2}{*}{ SS6 } & RMC (1min) (A) & 4862.5 & \multirow{2}{*}{ No incluida } & \multirow{2}{*}{ No incluida } \\
\hline & $\mathrm{RMC}(30 \mathrm{~min})(\mathrm{A})$ & 4032.7 & & \\
\hline \multirow{2}{*}{ SS7 } & RMC (1min) (A) & 4566.9 & 5164.9 & 5348.9 \\
\hline & $\mathrm{RMC}(30 \mathrm{~min})(\mathrm{A})$ & 3814.4 & 6227.1 & 6480.2 \\
\hline
\end{tabular}

Tabla 13 Tabla resumen resultados eléctricos escenarios estudiados

La evaluación de los resultados es necesario calcular la intensidad nominal para las subestaciones que se están analizando. De modo que, para subestaciones con 2 grupos de 3,5 MW, la potencia nominal será de $7000 \mathrm{KVA}$. La Intensidad por tanto:

$$
I_{n}=\frac{S_{n}}{V_{n}}=\frac{7000}{1500}=4666.66 \mathrm{~A}
$$

\section{Ecuación 1. Intensidad nominal de las subestaciones}

Como se puede comprobar en la Tabla 7, algunos de los valores superan la intensidad nominal. Para estos casos sería necesario un estudio de las curvas de carga de la subestación. Es una vía interesante de investigación el poder llegar a integrar un modelo de chequeo de posibles sobrecargas en subestación, aunque de momento todo esto se deberá realizar a posteriori en análisis del dimensionamiento eléctrico escogido. En el CASO siguiente si que se han realizado diversas pruebas de ciclos de carga de subestación, coincidiendo con la aplicación total de las posibilidades del método.

Trabajando con otras magnitudes eléctricas relevantes, la inserción de la restricción de situaciones degradadas simple y completa, hará que el algoritmo encuentre diseños más fiables y que den más seguridad acerca de la construcción final y eficacia en la operación real. Se verá en los próximos casos. 
En cuanto a los tiempos de resolución del algoritmo:

\begin{tabular}{lc}
\hline Situación & Tiempo(s) \\
\hline Tiempo medio por genotipo & 0.27 \\
Resolviendo sistema eléctrico & 0.11 \\
\hline
\end{tabular}

Tabla 14 Tiempos de cálculo operaciones del algoritmo NSGA-II CASO 1

Las pruebas se realizaron usando un computador que se podría catalogar de capacidad media-alta, y en ningún caso de altas prestaciones ni supercomputación. Las características básicas para medir su capacidad son:

- Procesador i5-2400

- $\quad 3,1 \mathrm{GHz}$ de velocidad de procesamiento

- 4 GB de memoria RAM

Los tiempos que ha tardado NSGA-II adaptado al sistema experto han variado entre la hora para el caso de los 10 individuos de población, hasta las casi 2 horas de la población con 20 individuos. Para poner en contexto el interés del trabajo, decir que una simulación completa de este escenario tiene una duración de 1h50'. Con esta duración si se quisiera seguir el proceso habitual, es obvio que habría que limitar esas pruebas. Con la metodología se pueden encontrar respuestas a las dudas que genera un proceso como este, en un corto espacio de tiempo. Aunque evidentemente, la mejora de las restricciones, grupos de transformación, etc, dará más robustez a las soluciones finales, como se demuestra en el CASO siguiente. 


\subsection{CASO 2: Línea de Metro. Subestaciones fijas. Uso tres técnicas optimización.}

\subsubsection{Infraestructura de la línea}

Línea de metro con 21 estaciones a lo largo de su recorrido. Al disponer de los datos originales del Proyecto, se han podido incluir las limitaciones de velocidad debidas a tramos donde es desaconsejable superar ese límite por motivos de seguridad de la circulación.

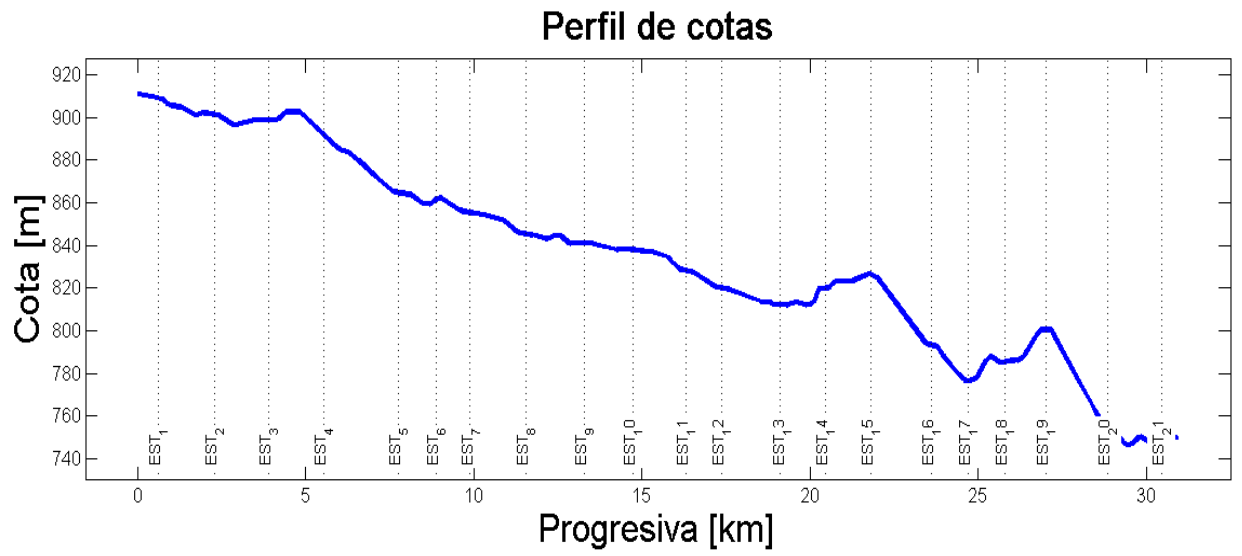

Figura 65 Perfil de alzado de línea para CASO 1

\subsubsection{Material rodante}

Configuración de la distribución de los coches del tren:

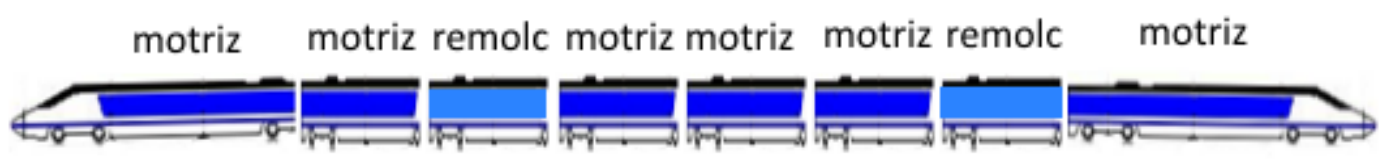

Figura 66 Ejemplo ilustrativo distribución coches en tren

En este caso el material rodante era de composición doble M-M-R-M-M-M-R-M Seis coches motrices y 2 restantes remolcados

\begin{tabular}{c|c}
\hline Propiedades & Valores \\
\hline Masa & $365.408 \mathrm{Tm}$ \\
Masas Giratorias & $36.54 \mathrm{Tm}$ \\
Pasajeros & 2064 \\
& $\mathrm{~A}[N]=7375.987$ \\
Coeficientes resistencia avance & $\mathrm{B}[N * \mathrm{~s} / \mathrm{m}]=167.039$ \\
& $\mathrm{C}\left[N * \mathrm{~s}^{2} / \mathrm{m}^{2}\right]=13.349$ \\
Resistencia en curva (Kc) & 500 \\
Deceleración emergencia & $1.5 \mathrm{~m} / \mathrm{s}^{2}$ \\
Deceleración servicio & $1.2^{\mathrm{m}} / \mathrm{s}^{2}$ \\
\hline
\end{tabular}




\begin{tabular}{c|c}
\hline Propiedades & Valores \\
\hline Aceleración tracción & $1^{\mathrm{m}} / \mathrm{s}^{2}$ \\
Aceleración confort (jerk) & $0.9 \mathrm{~m} / \mathrm{s}^{2}$ \\
Distancia deslizamiento & $4 \mathrm{~m}$ \\
Velocidad máxima & $80 \mathrm{~km} / \mathrm{h}$ \\
\hline
\end{tabular}

Tabla 15 Propiedades material rodante CASO 1

\subsubsection{Señalización}

Para este trabajo el sistema de señalización que se estableció para el estudio del sistema eléctrico, también fue CBTC.

\subsubsection{Sistema eléctrico inicial}

Las características principales del sistema eléctrico de la fase inicial son:

- Sistema de electrificación de corriente continua DC con tensión nominal de 3000V. Para este proyecto se ha establecido una tensión de vacío distinta de la nominal. Esta tensión de vacío aumenta en un $10 \%$ la nominal, la salida de tensión de la subestaciones será de 3300V.

- Los carriles son de tipo TR-57 de aleación de acero al carbono estándar. En un principio solo se contemplaba un tipo de catenaria (TIPO 1). Con las primeras pruebas de validación del escenario, se intentó paliar la sobretensión de retorno con la segunda catenaria TIPO 2, que incluye feeder de retorno.

\begin{tabular}{lllrl}
\hline \multicolumn{1}{c}{ Conductor } & Catenaria & Material & \multicolumn{1}{c}{ Sección } & Resistividad \\
\hline Sustentador & OHL 1/OHL 2 & Cobre & $261.5 \mathrm{~mm}^{2}$ & $0.018 \mu \Omega \cdot \mathrm{m}$ \\
Contacto & OHL 1/OHL 2 & Cobre & $2 \times 107.2 \mathrm{~mm}^{2}$ & $0.018 \mu \Omega \cdot \mathrm{m}$ \\
Carril estándar & OHL 1/OHL 2 & Acero & $7258 \mathrm{~mm}^{2}$ & $0.207 \mu \Omega \cdot \mathrm{m}$ \\
Feeder Retorno & OHL 2 & Cobre & $507 \mathrm{~mm}^{2}$ & $0.018 \mu \Omega \cdot \mathrm{m}$ \\
\hline
\end{tabular}

Tabla 16 Descripción catenarias CASO 2 corriente continua

En la configuración inicial se contemplaba la instalación de 4 subestaciones de tracción. Con las primeras pruebas se determinó que no eran suficientes. Para el proceso de optimización se trabaja directamente con 10 subestaciones, con las siguientes características: 


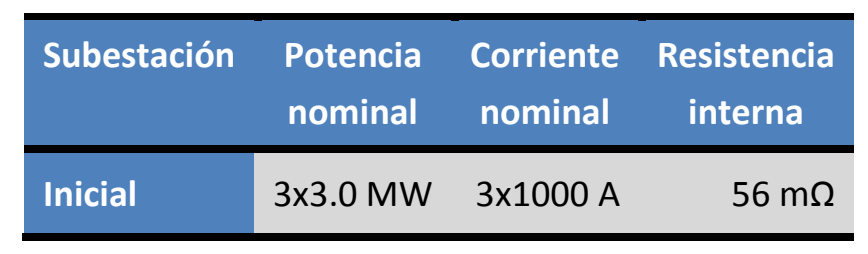

Tabla 17 Características básicas Subestaciones usadas en CASO 2

Escenario inicial configuración sistema eléctrico: Una de las características de este trabajo, era que las subestaciones ya tenían un sitio prefijado. Por lo que la posición es fija. Lo que el Método debe analizar es si la subestación es necesaria o no.

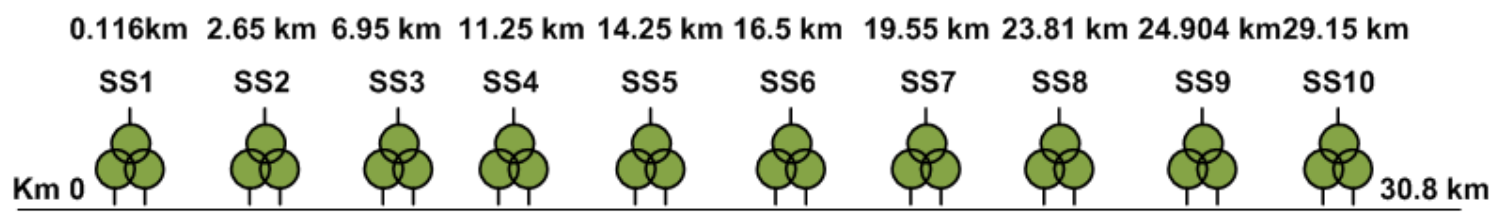

\section{Catenaria Tipo 1}

Figura 67 Configuración elementos sistema eléctrico inicial

\subsubsection{Plan de operación de la línea}

- Los trenes deben cumplir un intervalo entre ellos de 180s para situación normal de funcionamiento. Para situación de fallo doble de alguna subestación, se degrada la operación y el intervalo pasa a ser de 5 minutos.

- Paradas en estación de $20 \mathrm{~s}$.

- La flota calculada es de 29 trenes situación normal; 18 trenes situación degradada.

\subsubsection{Configuración algoritmo genético.}

Mismo procedimiento que para CASO 1.

\subsubsection{Genotipo}

El sistema presenta problemas de sobretensión en retorno en diversas zonas. Por este motivo, se ha intentado optimizar la catenaria por tramos. Tal y como se explicó en Capítulo 4, hará falta por tanto un gen para cada interzona de catenaria.

Para las subestaciones, 1 gen por cada una. Para algunas subestaciones, se intenta comprobar si podrían trabajar con menos potencia. Por lo que se integran en el genotipo los grupos de transformación. Las subestaciones que lo permiten son 5, por tanto, 5 genes más. El genotipo está formado por 25 componentes. En las pruebas será interesante contar cómo afecta esta gran cantidad al rendimiento temporal y a los resultados obtenidos. 


\begin{tabular}{|c|c|c|c|c|c|c|c|c|c|c|c|c|c|c|c|}
\hline $\mathrm{cat}_{1}$ & $\mathrm{cat}_{2}$ & $\mathrm{Cat}_{3}$ & $\mathrm{cat}_{4}$ & cat $_{5}$ & $\mathrm{Cat}_{6}$ & $\mathrm{Cat}_{7}$ & $\mathrm{cat}_{8}$ & cat $_{9}$ & $c a t_{10}$ & $S S_{1}$ & $s s_{2}$ & $S S_{3}$ & $S S_{4}$ & $S S_{5}$ & $S S_{6}$ \\
\hline$S S_{7}$ & $S S_{8}$ & $S S_{9}$ & $S S_{10}$ & $g r \_s s$ & $g r$ & & $S S_{3}$ & $g r_{-} S S_{4}$ & $g r_{-} S s_{5}$ & & & & & & \\
\hline
\end{tabular}

Figura 68 Distribución genes en Genotipo CASO 2

\subsubsection{Configuración para la Discretización Zonal y Ponderación de elementos}

La distribución de pesos asignados se muestra a continuación.

- Ponderación de los elementos significativos:

\begin{tabular}{|c|c|c|c|c|c|c|c|}
\hline Tipo Elemento & $O H L_{1}$ & $\overline{\mathrm{OHL}}$ & $g r_{-} s s_{1}$ & $g r_{-} s s_{2}$ & $g r_{-} s s_{3}$ & $g r_{-} s s_{4}$ & $g r_{-} s s_{5}$ \\
\hline Peso asignado & 300 & 500 & 2000 & 2000 & 2222 & 2222 & 2222 \\
\hline
\end{tabular}

Tabla 18 Pesos asignados a cada elemento

El peso de la catenaria 1 es más bajo porque analizando el material del que está formado y el precio, se ha estimado en un 66\% mayor el coste de una respecto de la otra. Las subestaciones se han valorado todas igual, por lo que no tiene influencia en la decisión su valoración directa. En cuanto a los grupos de subestaciones, decir que la inclusión de una modificación se le ha asignado un valor ponderado alto. La idea es influir lo menos posible en el dimensionamiento inicial en cuanto a la potencia de las subestaciones se refiere. En caso que tenga que introducir un cambio, estará justificado.

\begin{tabular}{|c|c|c|}
\hline $\begin{array}{l}\mathbf{N}^{0} \\
\text { Zona }\end{array}$ & Tipo Zona & $\begin{array}{l}\text { Peso } \\
\text { asignado }\end{array}$ \\
\hline Zona1 & \multirow{8}{*}{ Instalación } & 135 \\
\hline Zona2 & & 50 \\
\hline Zona3 & & 90 \\
\hline Zona4 & & 50 \\
\hline Zona5 & & 135 \\
\hline Zona6 & & 50 \\
\hline Zona7 & & 50 \\
\hline Zona8 & & 135 \\
\hline
\end{tabular}

Tabla 19 Información completa acerca de la Discretización Zonal elegida

Respecto a la discretización zonal, la subdivisión de las zonas de instalación y el número de ellas se corresponde con el algoritmo diseñado. Por cada subestación equivale una zona de instalación. Al tener una posición asignada, no tiene sentido asignar una zona más amplia. El algoritmo en este caso no trabaja con la longitud de la zona, solo con los pesos asignados.

El propósito de este proyecto se ha delimitado en encontrar un sistema eléctrico que cumpla con los requisitos normativos y operacionales. Con estas bases, la inclusión de los otros tipos de zonas para la evaluación, se ha descartado ya que no se disponía de ningún dato y no se quería desvirtuar el resultado final. Esta poca dispersión de ponderación y relativamente bajas posibilidades de modelar el sistema, usando la potencia del mismo en cuanto a otro tipo de zonas ya comentado, posición de las subestaciones, el repositorio de catenarias, hace que la diversidad se vaya a ver afectada, 
y en los resultados se conforme una homogeneidad en los genotipos-fenotipos finales obtenidos.

\subsubsection{Resultados eléctricos configuración inicial}

Tensiones extremas: Retorno

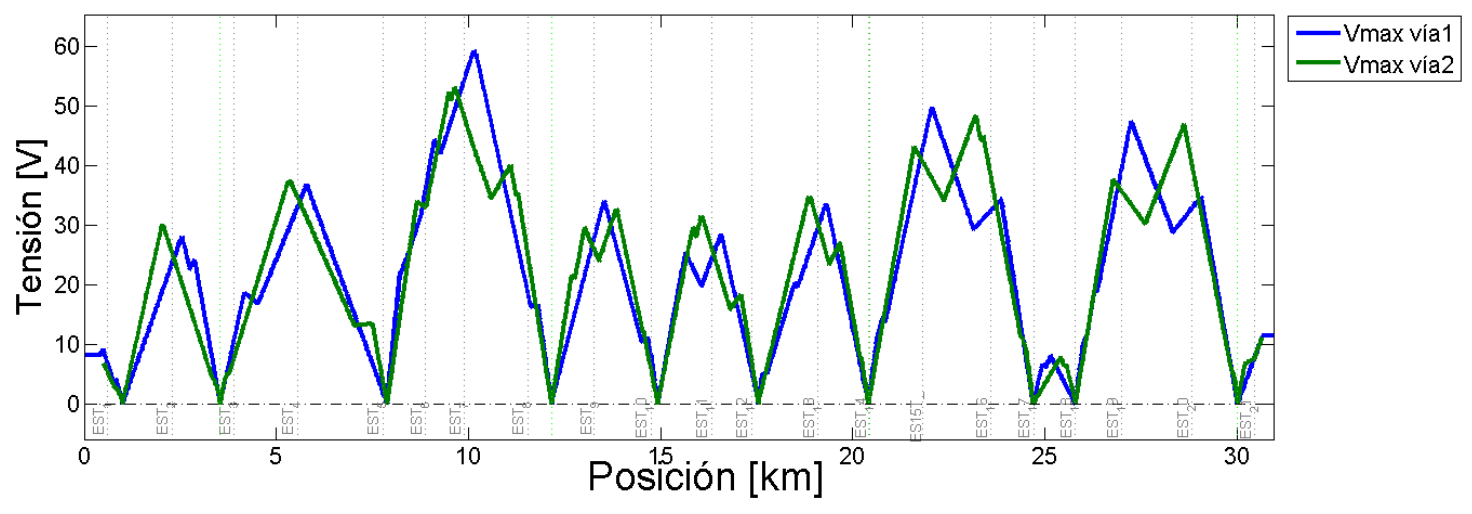

Figura 69 Tensiones máximas retorno carril-tierra

Tensiones mínimas: Catenaria

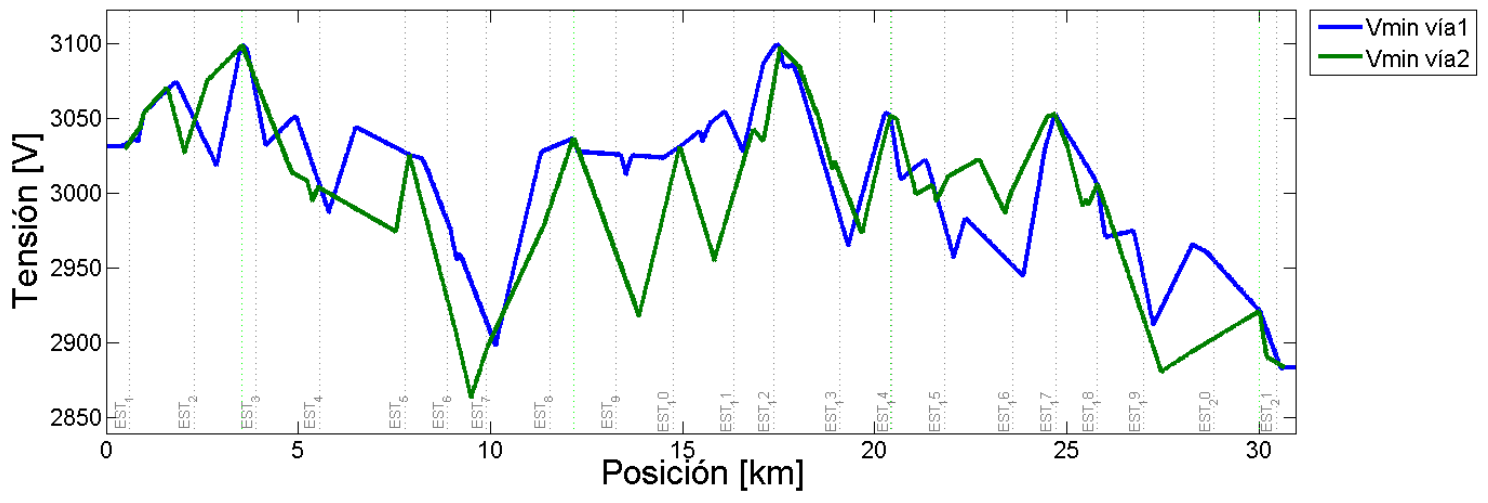

Figura 70 Tensiones mínimas en contacto catenaria-pantógrafo

Con las gráficas de tensiones se puede prever que pueden existir zonas de sobredimensionamiento eléctrico. Estas son las que el algoritmo debería encontrar y dar una solución alternativa que se ajuste a las necesidades reales y con buen valor de los fenotipos calculados.

\subsubsection{Proceso optimización del CASO 2. Configuración y resultados. Análisis.}

Los tres algoritmos genéticos finalmente elegidos para utilizar en las optimizaciones se han usado para hallar las mejores opciones en este proyecto. Trabajar con varios métodos de optimización va a permitir conocer el impacto de cada uno de ellos en la metodología, y poder establecer pautas de funcionamiento para próximas optimizaciones. Con este propósito, se ha pensado en una batería de pruebas, con distintos valores de configuración, teniendo en cuenta las características de cada algoritmo, de modo que con los resultados de cada una, se puedan extraer conclusiones.

El apartado siguiente se va a dividir en dos partes. En la primera se van a describir las pruebas realizadas para cada algoritmo. Los valores asignados a los parámetros de 
configuración importantes. La segunda parte consistirá en el análisis de los resultados obtenidos. Las conclusiones acerca de la evolución de las poblaciones con el transcurso de las generaciones, y los apuntes significativos de la generación final, y por tanto del frente de Pareto obtenido para los modelos aplicados.

\subsubsection{Configuración parámetros del algoritmo genético.}

Las pruebas realizadas han tratado de generar una serie de posibilidades diferentes para poder chequear el rendimiento de cada algoritmo y el comportamiento ante cambios en los valores de sus parámetros más importantes. Las distintas pruebas realizadas para cada algoritmo tienen su particularidad, que se mostrará en la descripción que viene a continuación. El factor sobre el que pivota el recorrido de los distintos casos de prueba va a ser el tiempo. En el detalle de las pruebas, se va a dividir por paquetes, donde cada cual pertenecerá a un conjunto de pruebas donde la respuesta de la optimización se prevé similar. El propósito debe estar encaminado a conseguir conocer el comportamiento de los algoritmos trasladados a optimizaciones como las que plantea el sistema eléctrico de esta línea ferroviaria. El siguiente parámetro importante a investigar es, lógicamente, el Frente de Pareto resultante, y la calidad del mismo.

\subsection{Espectro bajo de población y número de generaciones.}

Es interesante comprobar cómo afecta un número bajo de población y espacio evolutivo, a los algoritmos genéticos implementados. Esto conlleva que el número de variables sea relativamente alto, y con la complicación añadida de que en la mayoría de casos, los genes son de tipo Real, lo que dificulta el acceso al rango total del espacio del problema, y por tanto de añadir genotipos de distinta índole para que puedan ser analizados para su viabilidad por el fenotipo asociado.

De modo que el objetivo es ver cómo de buena puede ser la solución contando con un análisis rápido proporcionado por los algoritmos.

- $\varepsilon$-MOEA

○ Configuración

\begin{tabular}{l|ccc}
\hline Población & Prueba1 & Prueba2 & Prueba3 \\
\hline Población & 10 & 15 & 20 \\
No Eval & 100 & 100 & 150 \\
Épsilon & 1500 & 1500 & 1500 \\
Prob. Cruce & 0.8 & 0.8 & 0.8 \\
Prob. Mutación & 0.2 & 0.2 & 0.2 \\
Elim. Ss & -0.3 & -0.09 & -0.2 \\
\hline
\end{tabular}

Tabla 20 Configuración épsilon-MOEA nivel bajo 
○ Resultados

\begin{tabular}{lrccrrrc}
\hline & Fitness1 & Fitness2 & Tiempo & Rest1 & Rest2 & Rank1 & Rank2 \\
\hline Prueba1 & 625900 & 628147.9 & $25^{\prime}$ & $\square$ & $\mathbf{\square}$ & 1 & 3 \\
Prueba2 & 625900 & 628046.1 & 29 & $\square$ & $\square$ & 2 & 4 \\
Prueba3 & 501100 & 507024 & 32 & $\square$ & $\square$ & 7 & 4 \\
\hline
\end{tabular}

Tabla 21 Resultados épsilon-MOEA caso bajo

Las restricciones se refieren a la comprobación de si alguna de las restricciones no las cumple el/los individuo/s del frente de Pareto final.
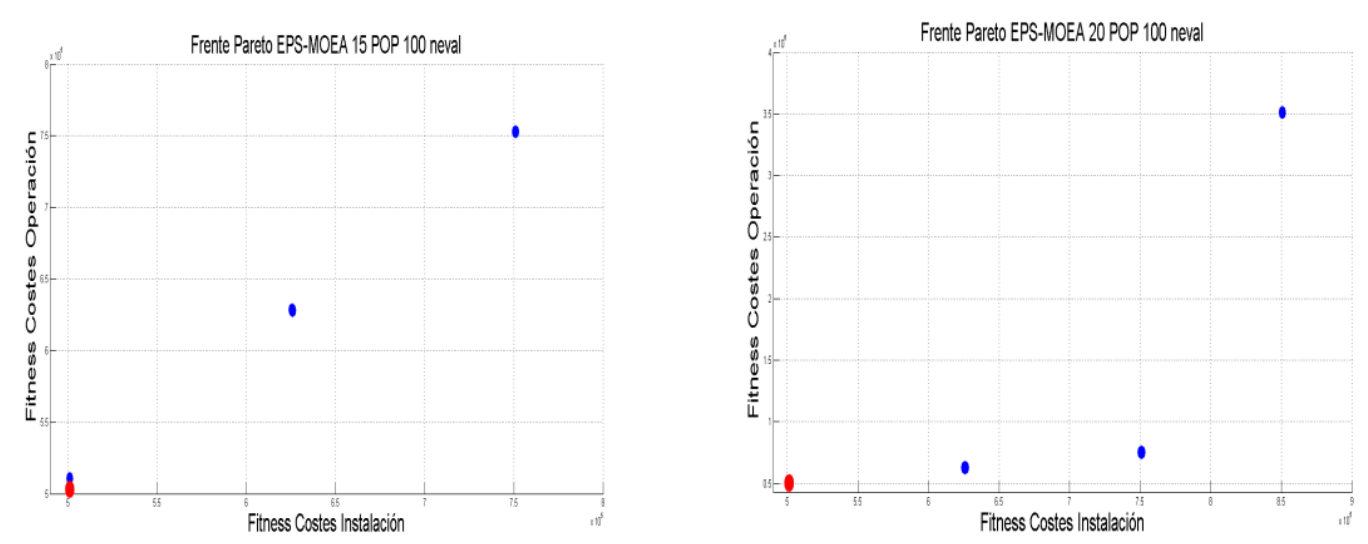

Figura 71 Frentes de Pareto. a) Prueba2;b) Prueba 3

Una de las características que se va a repetir en este ejemplo, es la poca diversidad y por otra parte, la consecución de un óptimo claro, reflejado en un Frente de Pareto compuesto por un solo valor. Esto es debido a la baja flexibilidad del sistema ferroviario estudiado y a las restricciones impuestas para validar el sistema eléctrico. No se permitían prácticamente reposicionamiento de subestaciones, y no se dispuso de información para realizar discretización zonal. En este caso se comprueba lo que se anticipaba, poca diversidad, aunque lo importante es que para valores pequeños de población y evaluaciones, parece que encuentra una configuración con un valor mínimo de las funciones objetivo, muy bueno en ambos casos.

\section{- AMGA-II}

○ Configuración

\begin{tabular}{lcccc}
\hline Población & Prueba1 & Prueba2 & Prueba3 & Prueba4 \\
\hline Población final & 15 & 15 & 25 & 25 \\
No Eval & 100 & 100 & 100 & 100 \\
Generaciones & 8 & 8 & 12 & 12 \\
Prob. Cruce & 0.9 & 0.75 & 0.9 & 0.75 \\
Prob. Mutación & 0.1 & 0.15 & 0.08 & 0.12 \\
Elim. Ss & -0.08 & -0.3 & -0.08 & -0.3 \\
\hline
\end{tabular}


Para este algoritmo, el número de individuos en la población final se establece por configuración, tal y como se comentó en el Capítulo 4, a partir de una población muy pequeña (para todos los casos 10), se van generando individuos hasta completar los deseados. Para la prueba en concreto se pensó en contrastar el funcionamiento del algoritmo para cambios en parámetros clave, como se puede ver en la tabla anterior. El problema es que la flexibilidad del ejemplo es muy baja, como ya se ha advertido en el ejemplo con el algoritmo MOEA.

\section{○ Resultados}

\begin{tabular}{lrrrrrrc}
\hline & Fitness1 & Fitness2 & Tiempo & Rest1 & Rest2 & Rank1 & Rank2 \\
\hline Prueba1 & 626100 & 628901.2 & 22 & $\mathbf{a}$ & $\mathbf{\square}$ & 1 & 1 \\
Prueba2 & 501100 & 507018.5 & 22 & $\mathbf{\square}$ & $\mathbf{\square}$ & 1 & 1 \\
Prueba3 & 501100 & 507061.8 & 25 & $\mathbf{\square}$ & $\mathbf{a}$ & 1 & 1 \\
& 626100 & 628153.8 & 25 & $\mathbf{\square}$ & $\mathbf{\square}$ & & \\
Prueba4 & 648320 & 628058.1 & 25 & $\mathbf{\square}$ & $\mathbf{\square}$ & 3 & 4 \\
& 625900 & 628583.3 & 25 & $\mathbf{\square}$ & $\mathbf{\square}$ & & \\
\hline
\end{tabular}

Tabla 23 Resultados épsilon-MOEA caso bajo
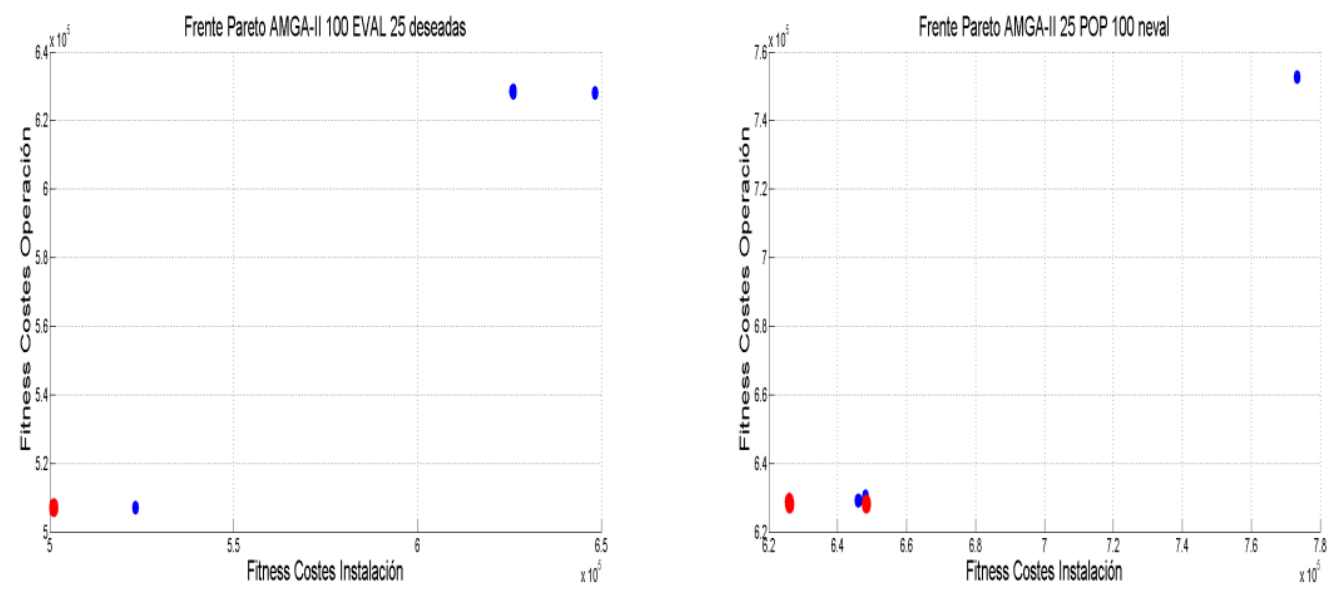

Figura 72 Frentes de Pareto. a) Prueba3;b) Prueba 4

Las pruebas 2 y 3 mejoran la primera debido al factor de eliminación de subestaciones y al mayor número de población deseada en la tercera. El caso extraño se encuentra en la prueba 4. No aparece ninguna señal que pueda derivar hacia una solución de los resultados obtenidos, ya que son peores que para el mismo caso de 15 población deseada. En próximas optimizaciones se tratará de buscar alguna respuesta, que no sea la de que la búsqueda heurística, no ha fluido por la rama óptima. En las gráficas aparece de nueva la falta de diversidad ya pronosticada. 
- NSGA-II

○ Configuración

\begin{tabular}{lcccc}
\hline Población & Prueba1 & Prueba2 & Prueba3 & Prueba4 \\
\hline Población final & 15 & 15 & 25 & 25 \\
Generaciones & 8 & 8 & 8 & 8 \\
Prob. Cruce & 0.7 & 0.7 & 0.7 & 0.7 \\
Prob. Mutación & 0.2 & 0.2 & 0.2 & 0.2 \\
Elim. Ss & -0.3 & -0.09 & -0.3 & -0.09 \\
\hline
\end{tabular}

Tabla 24 Configuración épsilon-MOEA nivel bajo

Con las pruebas realizadas en otros proyectos (ver CASOS 1 y 2 en Capitulo 6 y CASO 1 de este capítulo), se ha podido comprobar que el coste temporal de este algoritmo es mucho más alto que para los otros dos usados. Para este paquete de pruebas se ha decidido mantener las probabilidades de operar el cruce y mutación, ya que se quería mucha probabilidad de búsqueda por el espacio de soluciones.

○ Resultados

\begin{tabular}{cccccccc}
\hline & Fitness1 & Fitness2 & Tiempo & Rest1 & Rest2 & Rank1 & Rank2 \\
\hline Prueba1 & 626100 & 628034.1 & 45 & $\mathbf{a}$ & $\mathbf{\square}$ & 1 & 3 \\
Prueba2 & 626100 & 628728.7 & 46 & $\mathbf{\square}$ & $\mathbf{\square}$ & 15 & 0 \\
& 625900 & 628751.4 & 45 & $\mathbf{\square}$ & $\mathbf{\square}$ & & \\
Prueba3 & 625900 & 629386.6 & 55 & $\mathbf{\square}$ & $\mathbf{\square}$ & 2 & 3 \\
Prueba4 & 626100 & 628032 & 55 & $\mathbf{\square}$ & $\mathbf{\square}$ & 3 & 1 \\
\hline
\end{tabular}

Tabla 25 Resultados épsilon-MOEA caso bajo
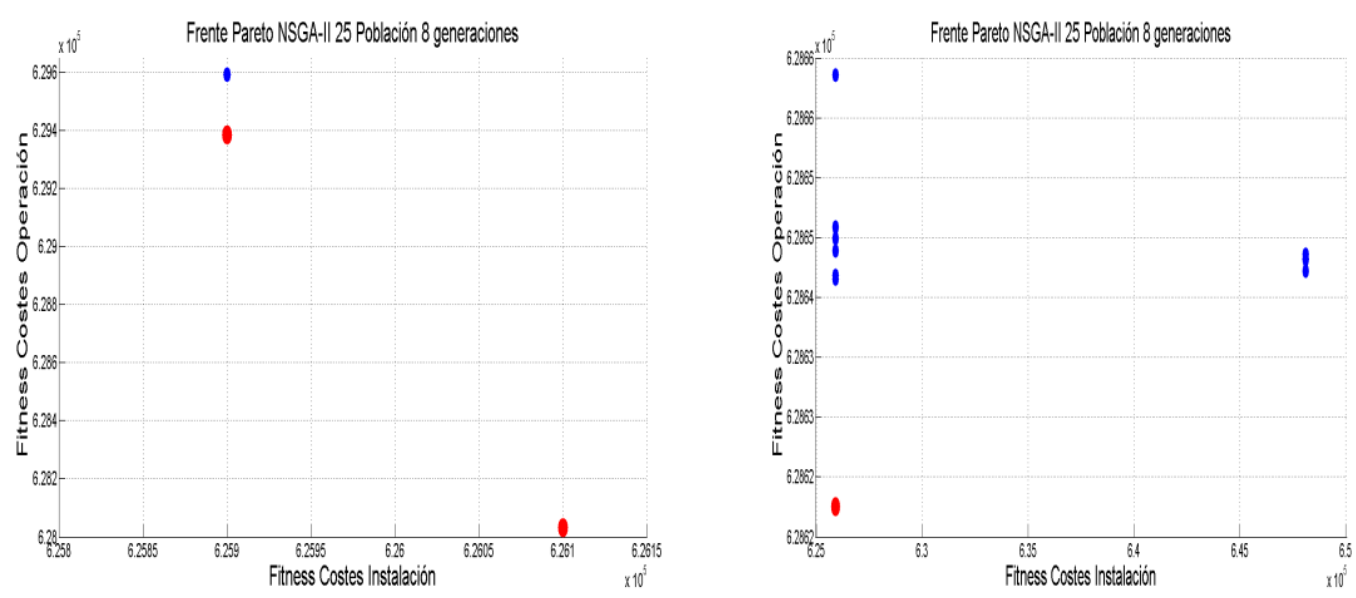

Figura 73 Frentes de Pareto. a) Prueba3;b) Prueba 4

El ránking muestra los individuos pertenecientes a cada espectro de soluciones. Para la Prueba 2, 15 individuos se situaron en el Frente de Pareto óptimo y 0 en el segundo escalón, aunque muchos tenían valores iguales. Esto es una diferencia clara respecto a los otros algoritmos, ya que cada individuo tiene una evolución diferenciada del resto, confluyendo en más homogeneidad, sobre todo con estas condiciones iniciales de 
proyecto. En cuanto a los resultados, los valores son bastante similares, sin acercarse al óptimo global, para ninguno de los dos valores.

\subsection{Espectro medio de población y número de generaciones.}

Recogiendo la experiencia obtenida en los estudios previos realizados solo con el algoritmo NSGA-II, se ha establecido este nivel medio de análisis por parte de los algoritmos genéticos.

\section{- $\quad$-MOEA}

○ Configuración

\begin{tabular}{lcccc}
\hline Población & Prueba1 & Prueba2 & Prueba3 & Prueba4 \\
\hline Población & 15 & 35 & 50 & 50 \\
No Eval & 500 & 200 & 200 & 200 \\
Épsilon & 500 & 500 & 1500 & 500 \\
Prob. Cruce & 0.8 & 0.8 & 0.8 & 0.8 \\
Prob. Mutación & 0.2 & 0.2 & 0.2 & 0.2 \\
Elim. Ss & -0.3 & -0.3 & -0.09 & -0.3 \\
\hline
\end{tabular}

Tabla 26 Configuración épsilon-MOEA nivel bajo

Se siguen manteniendo los valores de probabilidad de aplicar operadores.

○ Resultados

\begin{tabular}{lrccrrrc}
\hline & Fitness1 & Fitness2 & Tiempo & Rest1 & Rest2 & Rank1 & Rank2 \\
\hline Prueba1 & 500900 & 507086.7 & 70 & $\square$ & $\square$ & 1 & 2 \\
Prueba2 & 500900 & 507047 & 43 & $\square$ & $\square$ & 4 & 4 \\
Prueba3 & 625900 & 628045.4 & 54 & $\square$ & $\square$ & 1 & 8 \\
Prueba4 & 500900 & 507047 & 52 & $\square$ & $\square$ & 1 & 5 \\
\hline
\end{tabular}

Tabla 27 Resultados épsilon-MOEA caso bajo

En general, se puede confirmar que a partir de las 200 evaluaciones, el algoritmo encuentra un dimensionamiento válido, ya que las restricciones no se incumplen. Lo más significativo se encuentra en la Prueba3. Estos valores tan altos de Fitness, se producen debido a la incapacidad del algoritmo de eliminar de forma fácil subestaciones de tracción que provocan sobredimensionamiento. El bajo valor del parámetro Elim_ss, es el causante de este efecto.. 

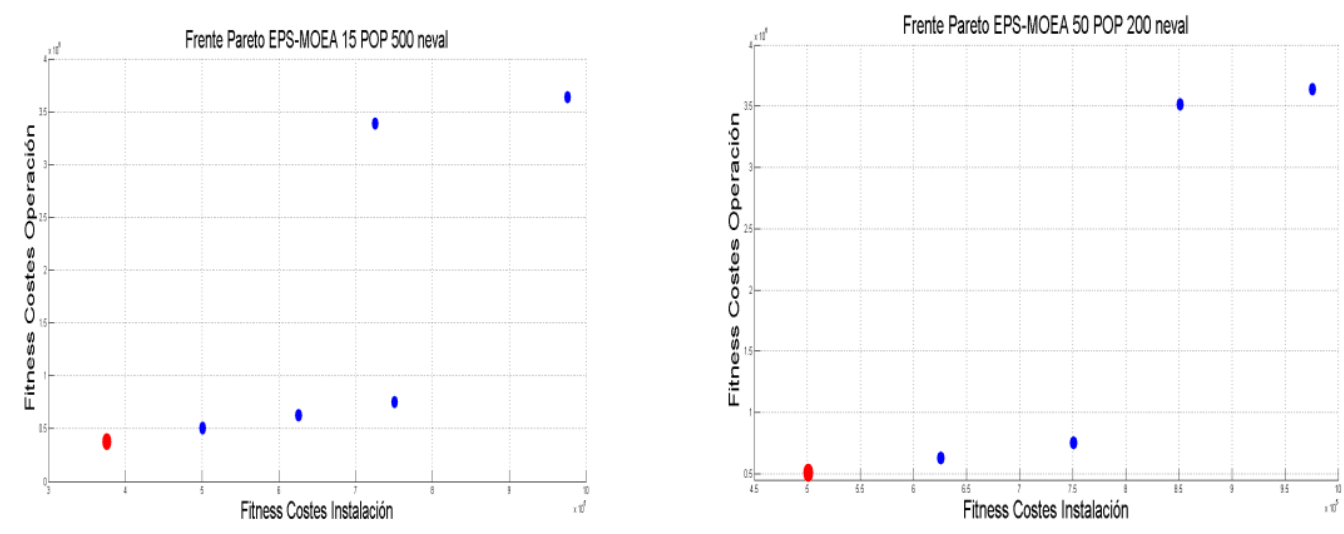

Figura 74 Frentes de Pareto. a) Prueba1;b) Prueba 3

En general se va a seguir con esta tónica en los diagramas de Frentes de la población final en todas las pruebas realizadas en el proyecto. Poca diversidad y muy concentrados los genotipos finales.

- AMGA-II

○ Configuración

\begin{tabular}{lcccc}
\hline Población & Prueba1 & Prueba2 & Prueba3 & Prueba4 \\
\hline Población final & 25 & 25 & 30 & 30 \\
$\mathbf{N}^{0}$ Eval & 250 & 250 & 250 & 250 \\
Generaciones & 26 & 26 & 12 & 12 \\
Prob. Cruce & 0.9 & 0.75 & 0.9 & 0.75 \\
Prob. Mutación & 0.1 & 0.1 & 0.15 & 0.15 \\
Elim. Ss & -0.08 & -0.3 & -0.09 & -0.3 \\
\hline
\end{tabular}

Tabla 28 Configuración épsilon-MOEA nivel bajo

La misma idea de comparar poblaciones similares con el límite de eliminación de subestación y la probabilidad de cruce, como mayores cambios entre ellas.

○ Resultados

\begin{tabular}{lccccccc}
\hline & Fitness1 & Fitness2 & Tiempo & Rest1 & Rest2 & Rank1 & Rank2 \\
\hline \multirow{3}{*}{ Prueba1 } & 648320 & 628275.4 & 35 & $\mathbf{a}$ & $\mathbf{\square}$ & & \\
& 625900 & 628672.3 & 35 & $\mathbf{\square}$ & $\mathbf{\square}$ & 3 & 2 \\
Prueba2 & 648120 & 628395.1 & $35^{\prime}$ & $\mathbf{\square}$ & $\mathbf{\square}$ & & \\
Prueba3 & 501100 & 507018.5 & 35 & $\mathbf{\square}$ & $\mathbf{\square}$ & 1 & 3 \\
Prueba4 & 501100 & 507338 & 39 & $\mathbf{\square}$ & $\mathbf{\square}$ & 1 & 2 \\
\hline
\end{tabular}

Tabla 29 Resultados épsilon-MOEA caso bajo 

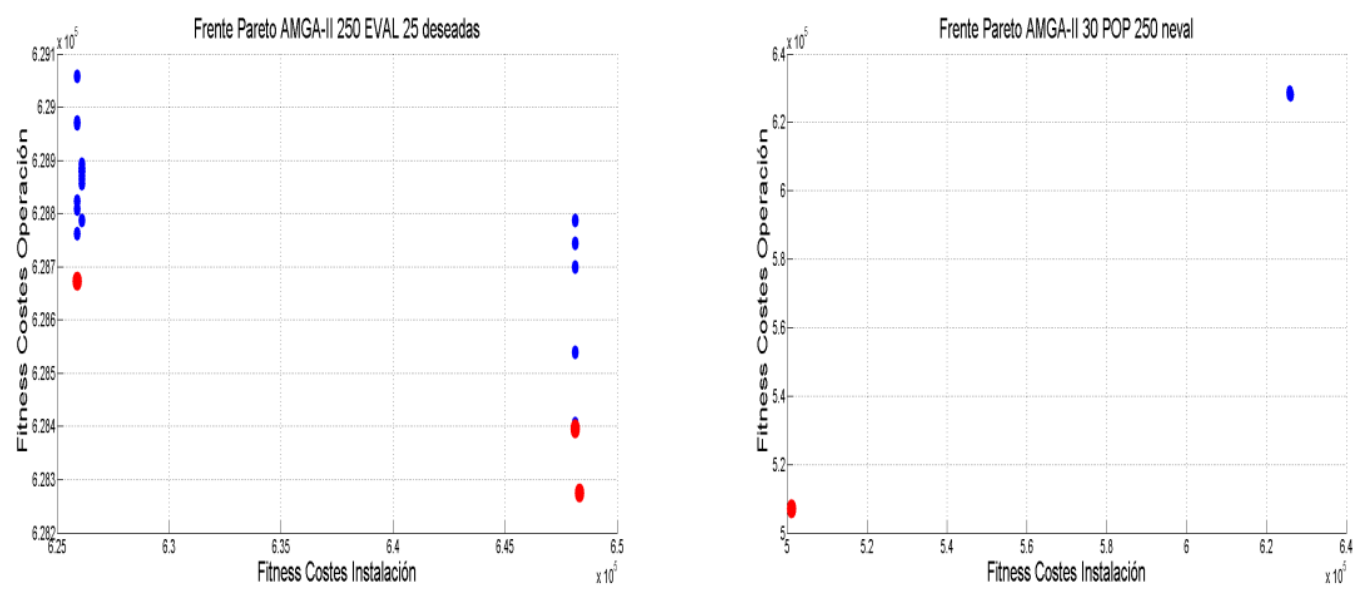

Figura 75 Frentes de Pareto. a) Prueba3;b) Prueba 4

Los resultados de estas pruebas confirmaron que para cortos espacios de decisión en cuento a poblaciones deseadas, el algoritmo puede dirigirse hacia un óptimo local, olvidando cualquier cambio genético que le lleve a uno global. La diversidad sigue dependiendo de la baja capacidad de maniobra para realizar cambios, y finalmente, se limita a pequeños cambios en las pérdidas de energía en la línea que afectan al fitness 2.

- NSGA-II

○ Configuración

\begin{tabular}{lcccc}
\hline Población & Prueba1 & Prueba2 & Prueba3 & Prueba4 \\
\hline Población final & 62 & 62 & 82 & 82 \\
Generaciones & 15 & 15 & 15 & 15 \\
Prob. Cruce & 0.7 & 0.7 & 0.7 & 0.7 \\
Prob. Mutación & 0.2 & 0.2 & 0.2 & 0.2 \\
Elim. Ss & -0.3 & -0.09 & -0.3 & -0.09 \\
\hline
\end{tabular}

Tabla 30 Configuración épsilon-MOEA nivel bajo

Aumenta significativamente la población y el número de generaciones.

○ Resultados

\begin{tabular}{|c|c|c|c|c|c|c|c|}
\hline & Fitness 1 & Fitness2 & Tiempo & Rest1 & Rest2 & Rank1 & Rank2 \\
\hline Prueba1 & 501100 & 508030 & $3 \mathrm{~h} 15^{\prime}$ & $\square$ & $\square$ & 1 & 1 \\
\hline Prueba2 & 501100 & 507058.15 & $3 \mathrm{~h} 21^{\prime}$ & च & $\mathbf{\square}$ & 1 & 2 \\
\hline Prueba3 & 501100 & 507027.28 & $4 h 35^{\prime}$ & 口 & 口 & 1 & 1 \\
\hline Prueha4 & 501100 & 507016.09 & $4 h 33^{\prime}$ & 口 & 口 & \multirow{2}{*}{3} & \multirow{2}{*}{3} \\
\hline & 500900 & 508390.4 & 4h 29' & [ & [ & & \\
\hline
\end{tabular}

Tabla 31 Resultados épsilon-MOEA caso bajo 

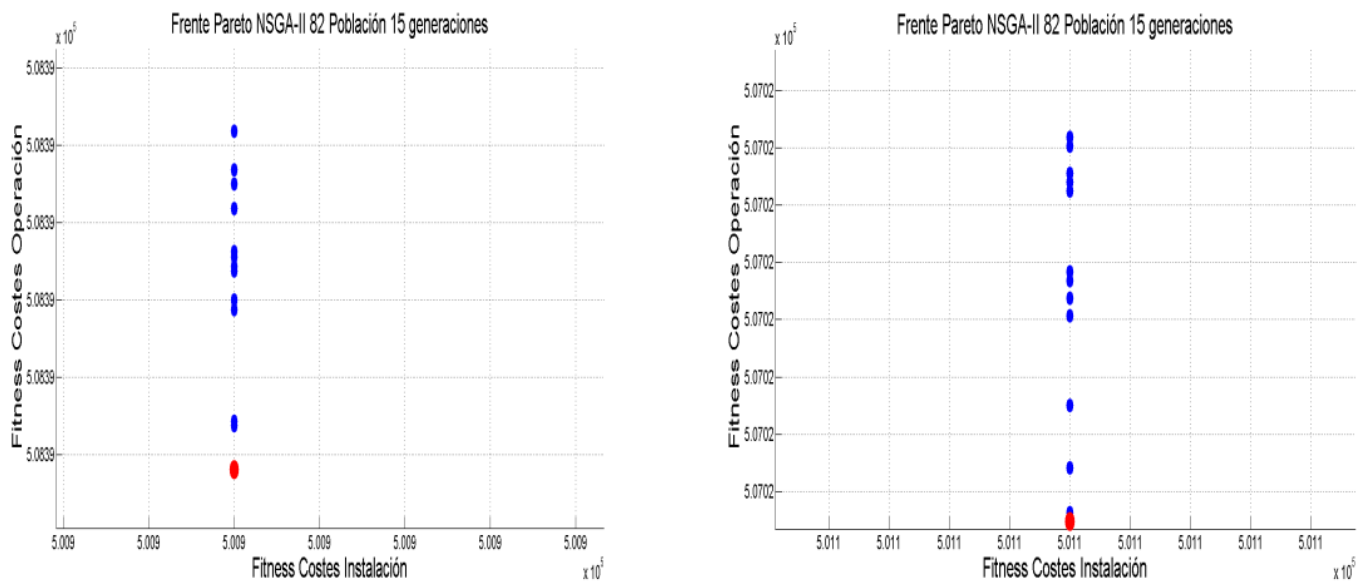

Figura 76 Frentes de Pareto. a) Rango sol1 Prueba4;b) Rango sol2 Prueba4

Significativo es el coste temporal de este algoritmo. Los resultados para la gama media de complejidad del problema ya se asemejan a los valores del óptimo global encontrados en casos anteriores. Se ha graficado en esta ocasión, las dos zonas donde pivotan las soluciones del Frente de Pareto para la prueba4, demostrando una metodología similar a las anteriores.

\subsection{Espectro alto de población y número de generaciones.}

Finalmente, se comprueba si para un gran espectro de búsqueda y de evolución, el impacto en la solución final realmente mejora las prestaciones de los grupos anteriores. Como se ha podido constatar anteriormente, para este CASO, al tener ciertas restricciones y limitaciones en cuanto a la capacidad de variar el dimensionamiento eléctrico, se tenía la incertidumbre de si ya se había alcanzado el óptimo en las pruebas precedentes.

- $\varepsilon$-MOEA

○ Configuración

\begin{tabular}{lcccc}
\hline Población & Prueba1 & Prueba2 & Prueba3 & Prueba4 \\
\hline Población & 80 & 120 & 150 & 180 \\
$N^{0}$ Eval & 500 & 500 & 600 & 700 \\
Épsilon & 3000 & 500 & 1500 & 500 \\
Prob. Cruce & 0.8 & 0.8 & 0.8 & 0.8 \\
Prob. Mutación & 0.2 & 0.2 & 0.2 & 0.2 \\
Elim. Ss & -0.09 & -0.3 & -0.09 & -0.3 \\
\hline
\end{tabular}

Tabla 32 Configuración épsilon-MOEA nivel bajo

○ Resultados

\begin{tabular}{|c|c|c|c|c|c|c|c|}
\hline & Fitness 1 & Fitness2 & Tiempo & Rest1 & Rest2 & Rank1 & Rank2 \\
\hline Prueba1 & 500900 & 508300.9 & $63^{\prime}$ & 口 & $\bar{\square}$ & 1 & 2 \\
\hline Prueba2 & 500900 & 507044.4 & $67^{\prime}$ & $\mathbf{\square}$ & 口 & 1 & 2 \\
\hline Prueba3 & 500900 & 507040.7 & $73^{\prime}$ & 口 & 口 & 1 & 3 \\
\hline
\end{tabular}




\begin{tabular}{lrccrrrr}
\hline & Fitness1 & Fitness2 & Tiempo & Rest1 & Rest2 & Rank1 & Rank2 \\
\hline Prueba4 & 500900 & 507039.4 & $80^{\circ}$ & - & a & 4 & 3 \\
\hline
\end{tabular}

Tabla 33 Resultados épsilon-MOEA caso bajo

Las restricciones se refieren a la comprobación de si alguna de las restricciones no las cumple el/los individuo/s del frente de Pareto final.

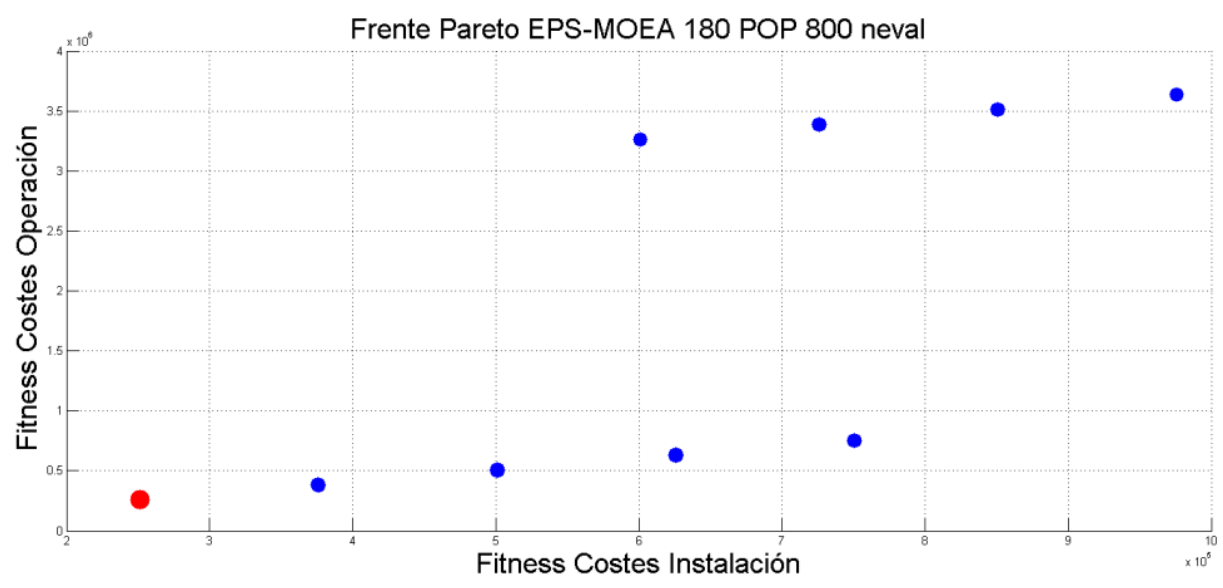

Figura 77 Frente de Pareto Prueba4

Los cambios en la configuración, y la mayor búsqueda por el espacio de soluciones debido al mayor número de individuos, no inciden en una mejora sustancial. De hecho, para este caso de poca flexibilidad, se puede afirmar que para un término medio de individuos y población, se alcanza más rápidamente un resultado satisfactorio, desaconsejando continuar con exámenes más costosos temporalmente.

- AMGA-II

○ Configuración

\begin{tabular}{lcc}
\hline Población & Prueba1 & Prueba2 \\
\hline Población final & 120 & 120 \\
N$^{0}$ Eval & 1500 & 1500 \\
Generaciones & 152 & 152 \\
Prob. Cruce & 0.76 & 0.9 \\
Prob. Mutación & 0.15 & 0.15 \\
Elim. Ss & -0.3 & -0.09 \\
\hline
\end{tabular}

Tabla 34 Configuración épsilon-MOEA nivel bajo

Por último, se trata de buscar un amplio margen del espacio de soluciones para ver el comportamiento del algoritmo. Modificaciones como siempre en probabilidad de cruce y eliminación de subestación.

○ Resultados

\begin{tabular}{lrccrrrc}
\hline & Fitness1 & Fitness2 & Tiempo & Rest1 & Rest2 & Rank1 & Rank2 \\
\hline Prueba1 & 500900 & 507038.2 & 93 & 口 & 口 & 2 & 3 \\
\cline { 2 - 7 }
\end{tabular}




\begin{tabular}{rrrrrrrr}
\hline & Fitness1 & Fitness2 & Tiempo & Rest1 & Rest2 & Rank1 & Rank2 \\
\hline \multirow{5}{*}{ Prueba2 } & 501100 & 507011.1 & 94 & $\mathbf{a}$ & $\mathbf{\square}$ & & \\
& 500700 & 508379.6 & 94 & $\mathbf{a}$ & $\mathbf{\square}$ & & \\
& 500900 & 5070403.3 & 93 & $\mathbf{a}$ & $\mathbf{\square}$ & 3 & 2 \\
& 501100 & 507012.9 & 93 & $\mathbf{\square}$ & $\mathbf{\square}$ & & \\
\hline
\end{tabular}

Tabla 35 Resultados épsilon-MOEA caso bajo
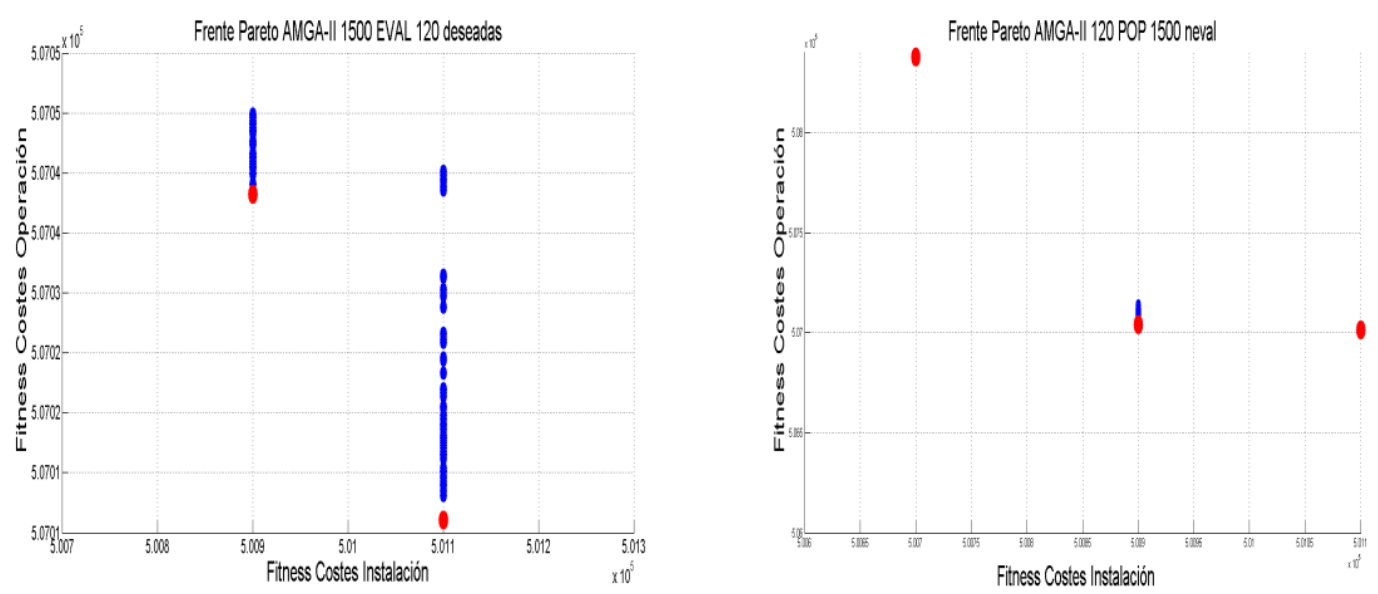

Figura 78 Frentes de Pareto. a) Prueba3;b) Prueba 4

En este caso si que se aprecian novedades. La más significativa es el caso 1 de la Prueba2, el fenotipo para la función objetivo 1 es el mejor obtenido. De igual manera, para el caso 2 de la prueba 1, el fitness de la función de costes de instalación es el más bajo de todos. Aumentar en gran número el espacio de búsqueda si ha dado resultado. El tiempo de espera no es muy importante, ya que con poco más de hora y media se obtiene una solución al dimensionamiento. En cuanto a las gráficas, se mantiene la misma tónica de agruparse por cercanía al mejor valor de costes de operación, que parece fijar el resto de soluciones posibles.

- NSGA-II

○ Configuración

\begin{tabular}{lc}
\hline Población & Pruebal \\
\hline Población final & 150 \\
Generaciones & 20 \\
Prob. Cruce & 0.7 \\
Prob. Mutación & 0.2 \\
\hline Elim. Ss & -0.3 \\
\hline
\end{tabular}

Tabla 36 Configuración épsilon-MOEA nivel bajo

El coste temporal del algoritmo, es progresivamente incrementado conforme se aumenta el número de población que interviene. Se ha probado un solo caso, para verificar el comportamiento del algoritmo, sin dar más relevancia a la influencia de los demás parámetros. Con la configuración dada, se busca gran diversidad. 
○ Resultados

\begin{tabular}{rrrrrrrc}
\hline & Fitness1 & Fitness2 & Tiempo & Rest1 & Rest2 & Rank1 & Rank2 \\
\hline Prueba1 & 501100 & 507020 & $9 \mathrm{~h} \mathrm{41}$ & $\mathbf{0}$ & $\mathbf{0}$ & 13 & 76 \\
\hline
\end{tabular}

Tabla 37 Resultados épsilon-MOEA caso bajo

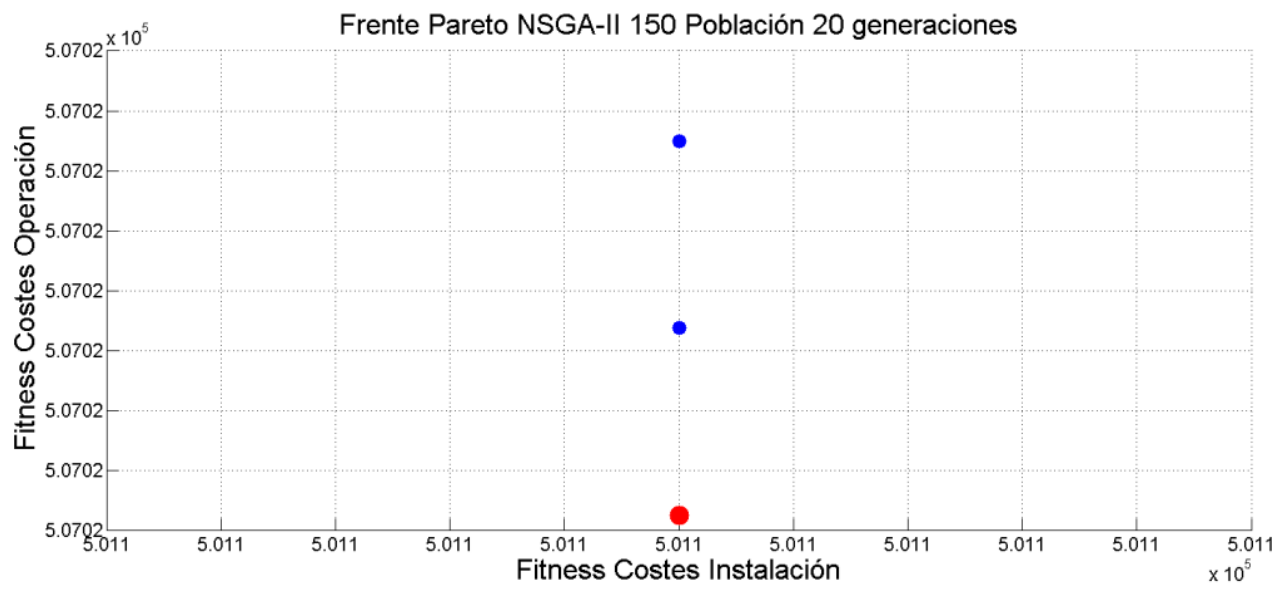

Figura 79 Frentes de Pareto de la Prueba1

El resultado final contiene buenos valores tanto para el fitness1 como para el fitness2, aunque en ninguno de los casos es el mejor de los encontrados y tabulados en las distintas pruebas realizadas. Se percibe el desarrollo del algoritmo, como se concentra en los individuos generados inicialmente y como van evolucionando sin tener en cuenta el impacto sobre los demás, así se llega a tener 13 individuos con el mismo fenotipo, y en ránking2, 76. El coste temporal, es mucho mayor que las otras dos técnicas de optimización, pero aun así, no es inasumible.

\subsection{Conclusiones.}

En general se ha ido comentando los distintos resultados aplicados a cada algoritmo. Está claro que el beneficio obtenido por usar el NSGA-II en estas condiciones queda muy diluido al compararlo con los otros dos. Mucho más tiempo de cálculo y las soluciones finales son prácticamente iguales. Una consecuencia a extraer es que para modelos con escasa flexibilidad, es muy útil usar el algoritmo MOEA o el AMGA, ya que son mucho más rápidos y obtienen una solución muy cercana al óptimo global, como se ha demostrado con los conjuntos de pruebas realizados.

Para el análisis eléctrico de las soluciones aportadas por los algoritmos, se ha fijado la atención en un par de configuraciones pertenecientes a los frentes de Pareto. La mayor diferencia entre ambas es la eliminación de subestaciones distintas, por lo que añade un factor de comprobación muy interesante para afinar correctamente las condiciones del sistema eléctrico final. 


\subsubsection{Diseño dimensionamiento con eliminación de dos subestaciones de tracción.}

Esta ha sido la configuración más repetida en los Frentes de Pareto finales obtenidos. En concreto el algoritmo eliminaba dos subestaciones centrales, que en el proyecto inicial no son de las ya construidas y en operación.

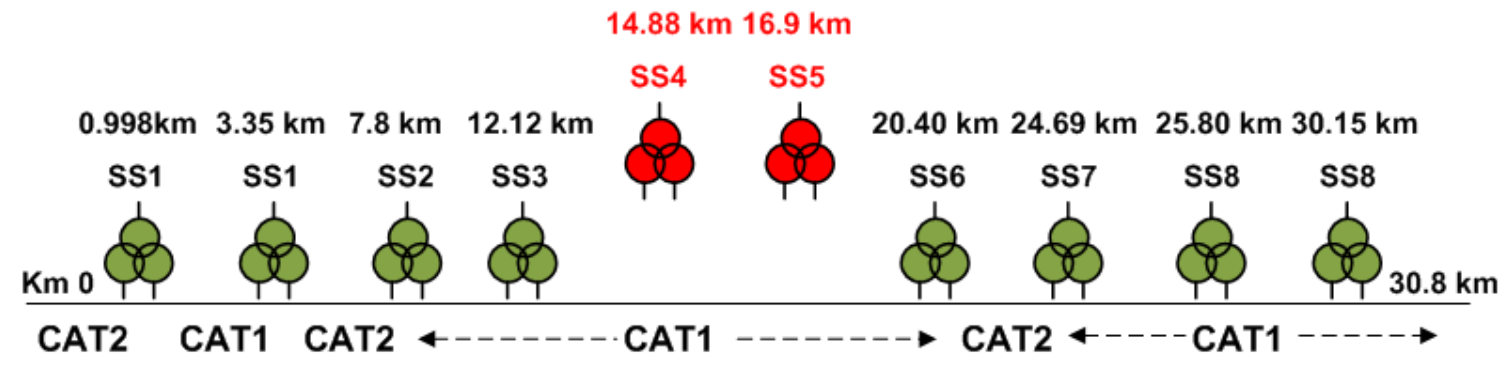

Figura 80 Escenario ejemplo 1 casos optimización Frente Pareto

En cuanto a los grupos de transformación, el genotipo no realiza cambios salvo para la subestación del pk 30.15, que deja la cantidad de grupos en 2, en vez de los tres con los que estaba dimensionado inicialmente.

El trabajo de análisis del sistema eléctrico conlleva la comprobación de muchos factores. En este apartado se ha tratado de escoger las situaciones degradadas que, por la situación y análisis previo, se presumía podía dar más problemas de viabilidad. El planteamiento pues, es mostrar las gráficas de tensiones mínimas en pantógrafo y de retorno carril-tierra para la situación normal del escenario optimizado, para a continuación mostrar los resultados más relevante de las situaciones degradadas complejas desde el punto de vista de estabilidad del sistema.

\section{- Funcionamiento normal}
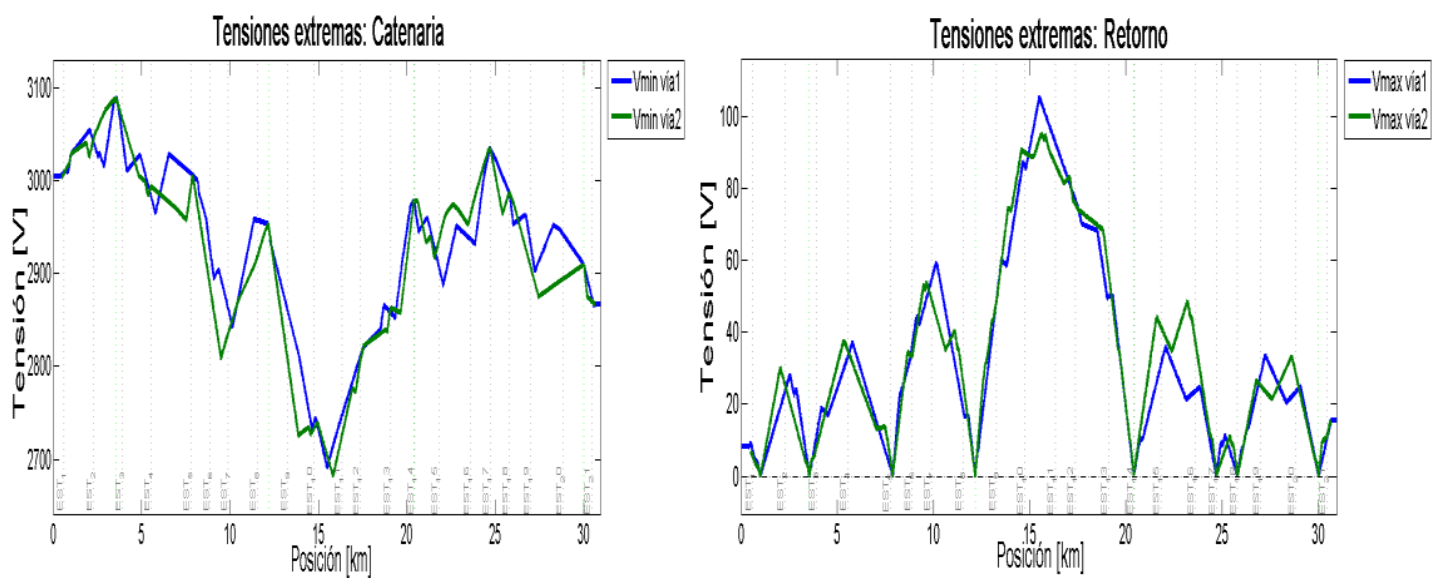

Figura 81 Ejemplo 1 Funcionamiento normal. a) tensiones mínimas catenaria;b) tensiones máximas retorno

Donde antes estaban las subestaciones, ahora al ser eliminadas se advierte una caída más pronunciada en el potencial, y en retorno una subida cercana a los 100V. Este primer análisis de la situación para el nuevo dimensionamiento, coloca ese punto como 
el más crítico. Va a ser objeto de estudio este y los extremos, siempre muy sensibles a situaciones degradadas, debido a no tener subestaciones adyacentes por una parte y en ocasiones, a operaciones de cambios de cambio de sentido, con grandes esfuerzos de tracción.

- Situaciones degradada simple, o caída de un grupo en la subestación
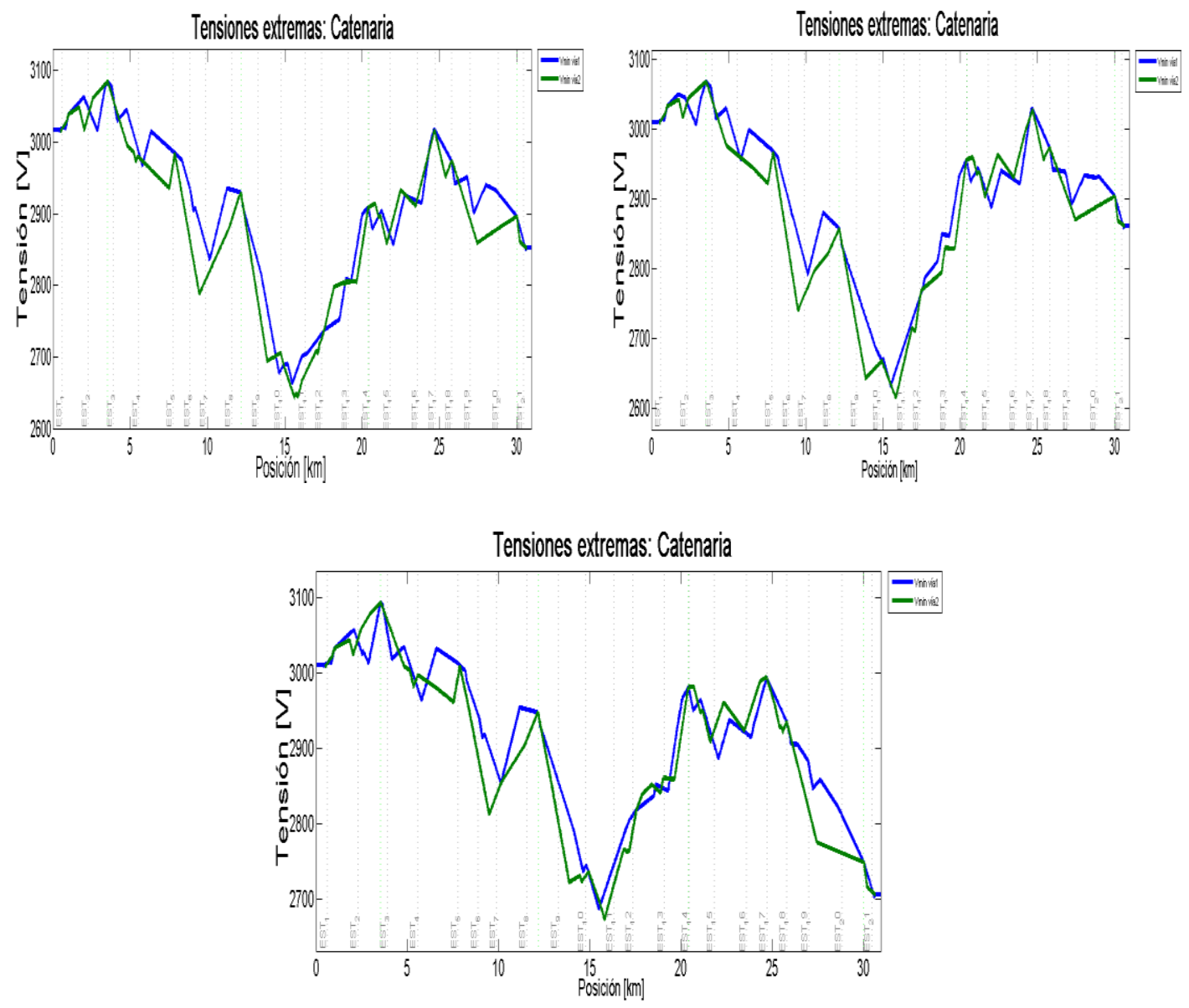

Figura 82 Tensiones mínimas catenaria. a) fallo simple ss7; b) fallo simple ss4; c) fallo simple ss10

La tensión mínima se sitúa alrededor de los $2600 \mathrm{~V}$ para el caso b), muy lejos del mínimo permitido de $2100 \mathrm{~V}$.
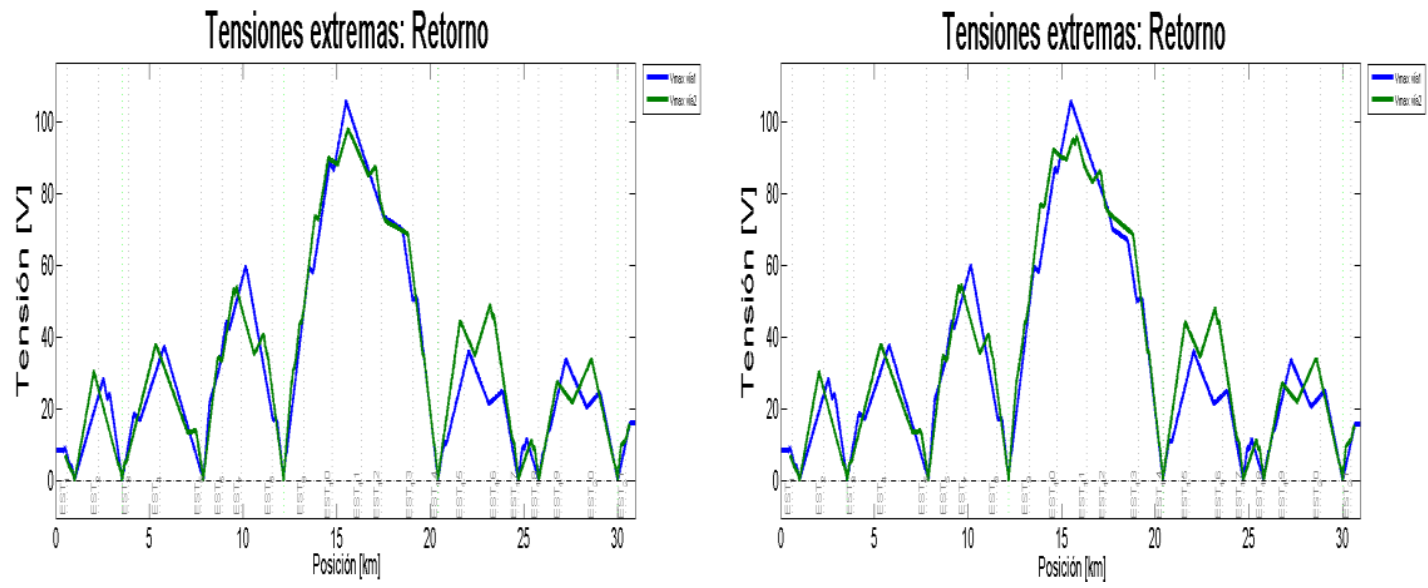


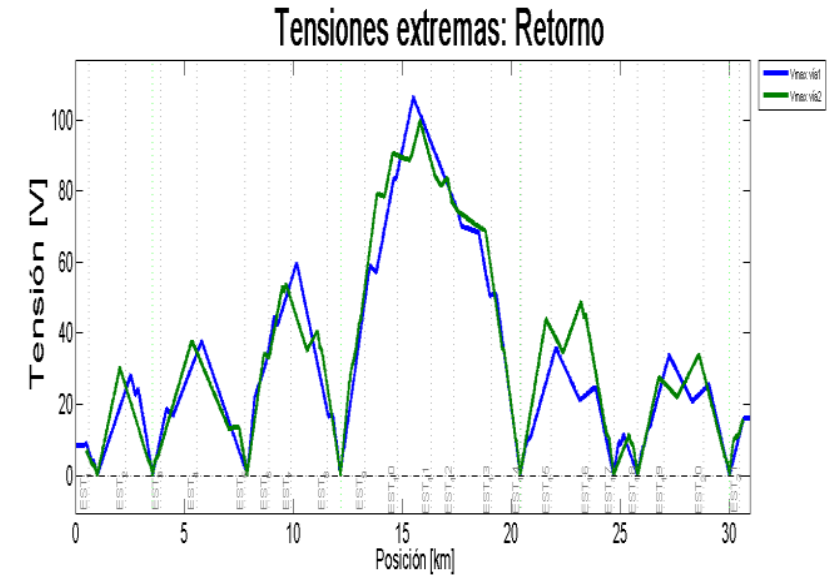

Figura 83 Tensiones de retorno para los tres casos mencionados anteriormente

La tensión máxima se sitúa alrededor de los 105V, no superando el límite de los $120 \mathrm{~V}$, a partir del cual se debería estudiar el transitorio para 5minutos. 


\section{- Situaciones degradadas doble o fuera de servicio una subestación}

Las situaciones críticas examinadas son las mismas que para el fallo simple. Para esta parte se ha añadido el caso de la otra subestación terminal.
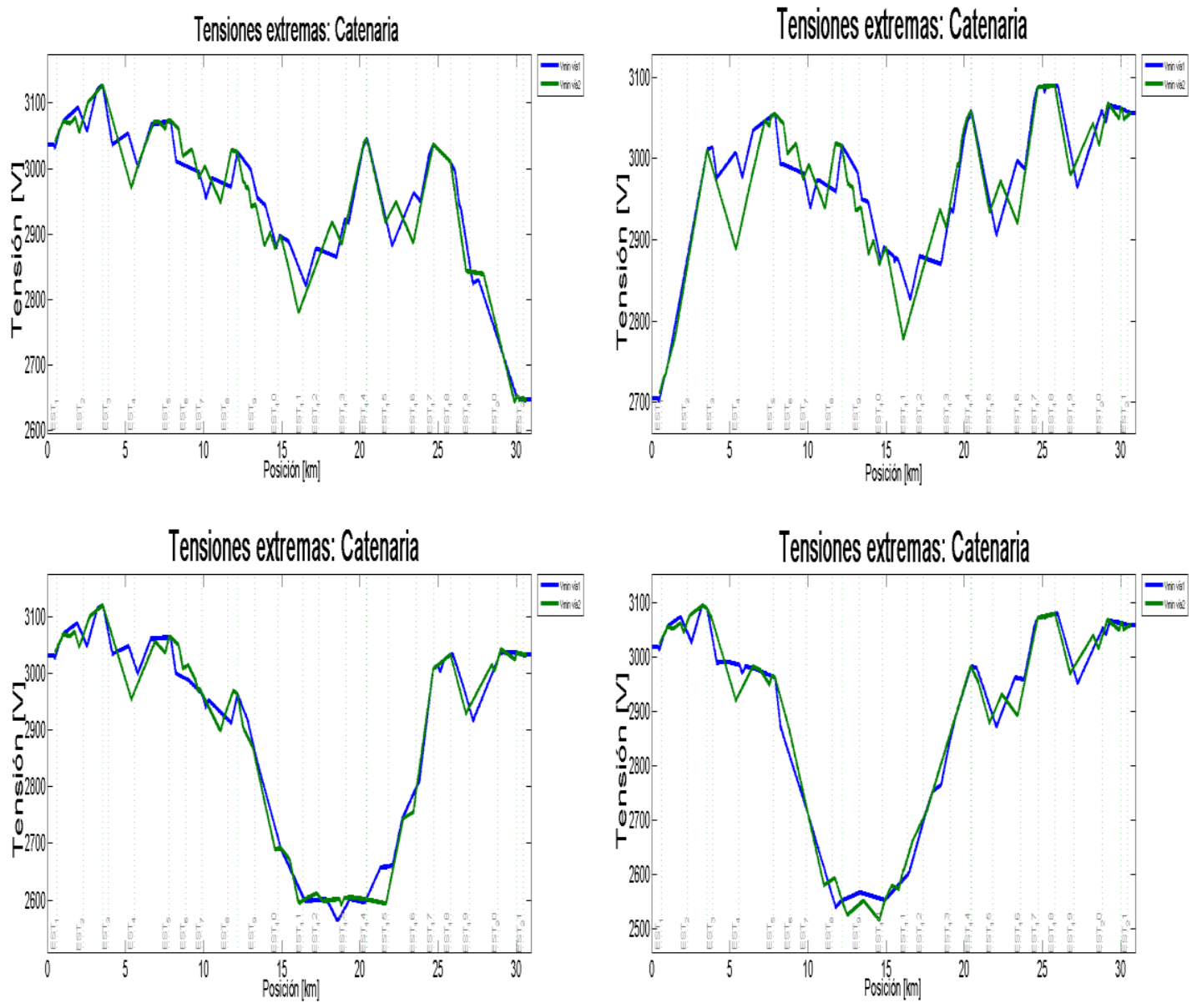

Figura 84 Fallo completo subestación. a) ss10; b) ss1;c) ss4; d) ss7 

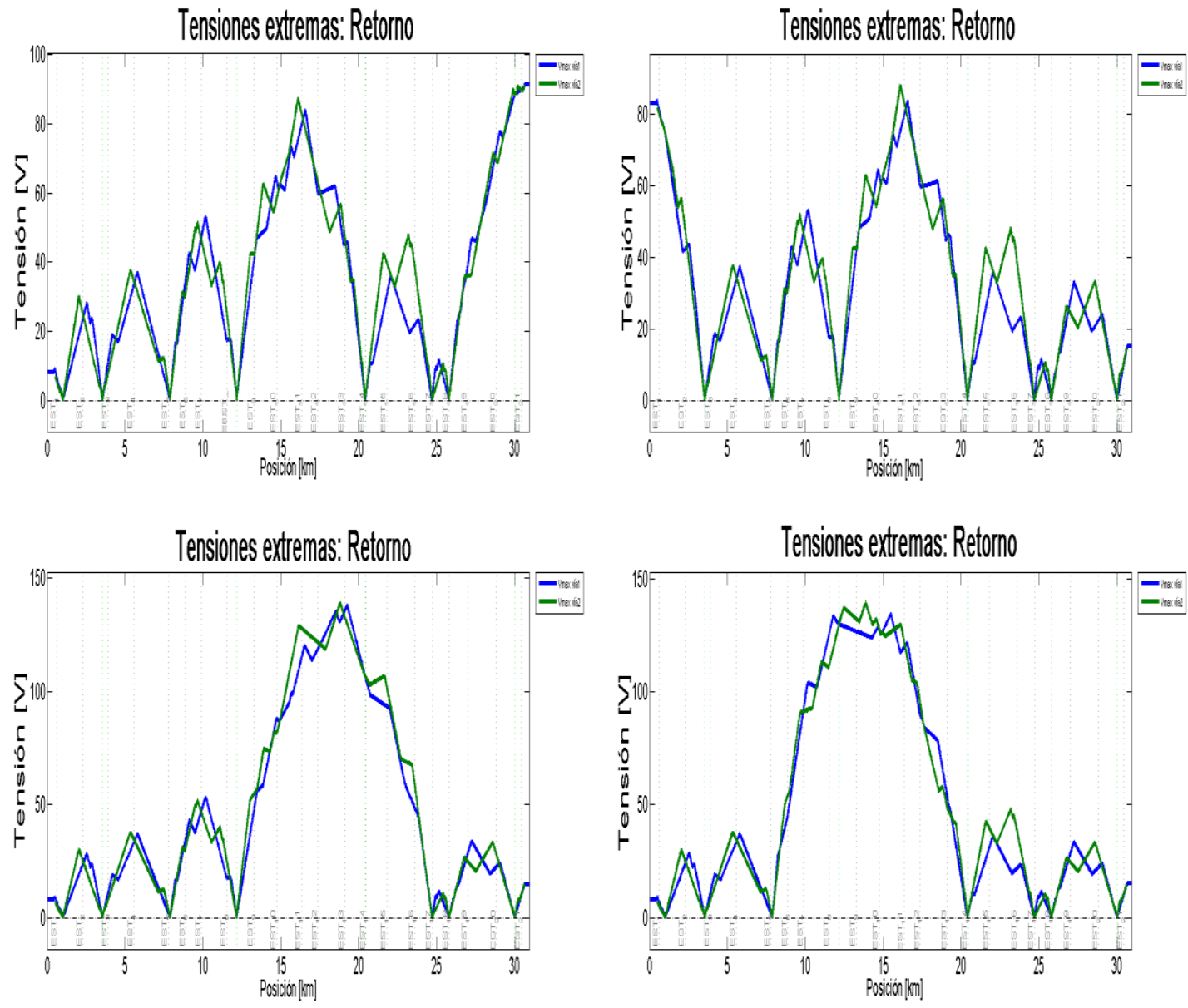

Figura 85 Tensiones máximas de retorno para los casos elegidos

Por último se presentan los valores de intensidades medias cuadráticas, que permiten evaluar si una subestación puede llegar a tener problemas de sobrecarga.

\begin{tabular}{lllll}
\hline $\begin{array}{l}\text { Fallo } \\
\text { simple }\end{array}$ & Normal & SS4 & SS7 & SS10 \\
\hline SS1 & 1271 & 1301 & 1276 & 1273 \\
SS2 & 1889 & 1958 & 1901 & 1893 \\
SS3 & 2373 & 2593 & 2407 & 2377 \\
SS4 & 2801 & 2323 & 2923 & 2814 \\
SS7 & 2700 & 2823 & 2229 & 2763 \\
SS8 & 2100 & 2134 & 2276 & 2299 \\
SS9 & 1496 & 1512 & 1579 & 1702 \\
SS10 & 1606 & 1615 & 1650 & 1146 \\
\hline
\end{tabular}

Tabla 38 Irms a 3600s para los casos estudiados en fallo simple

En naranja se han destacado las situaciones que estarían por encima de la intensidad nominal de las subestaciones, 1000 y 2000 A respectivamente. En esta situación hay que estudiar el ciclo de carga para conocer si existe o no sobrecarga. 
Los criterios de carga para los grupos rectificadores deben cumplir con el siguiente ciclo:

○ $100 \%$ en régimen permanente.

- $150 \%$ durante 2 horas, con intervalo de 3 horas.

○ $300 \%$ durante 1 minuto, con intervalo de 30 minutos.

La siguiente figura muestra la curva de duración de carga límite para tres grupos de 3.0 MW; la curva de cada subestación será proporcional según el número de grupos que tenga en paralelo.

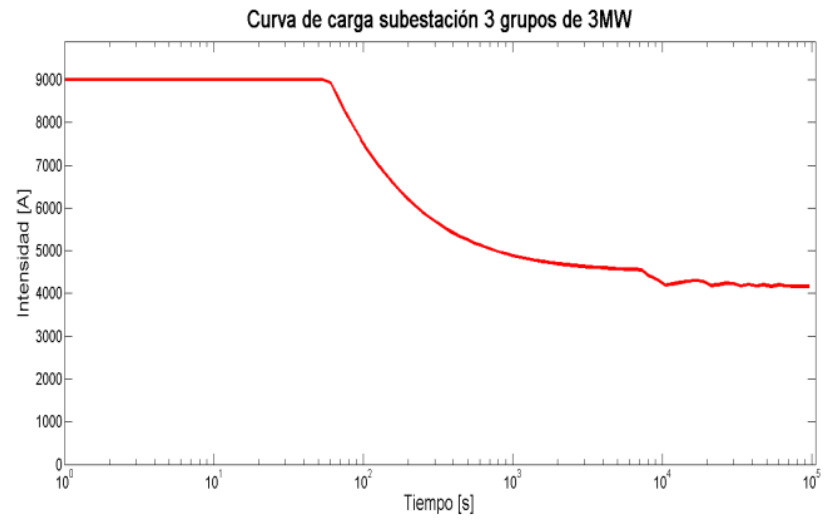

Figura 86 Curva de carga para subestación 3 grupos de 3 MW

Se calculan las curvas de carga, cuyo resultado se muestra a continuación: 

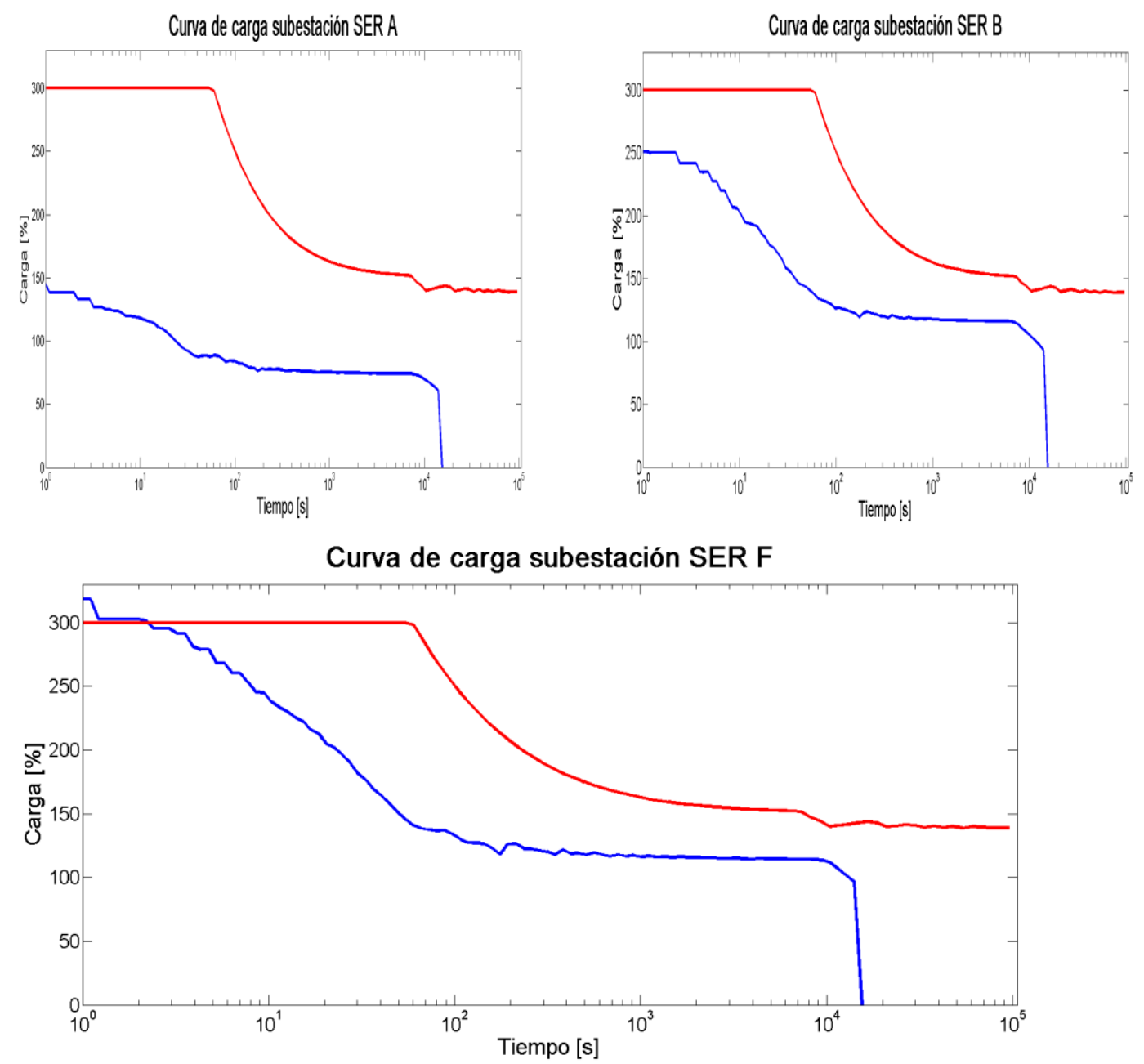

Figura 87 Curvas de sobrecarga para estudio degradadas simple. a) caso deg simple SS4 estudio SS7; b) caso deg simple SS7 estudio SS5; c) caso deg simple SS10 estudio SS10

Representando la línea azul la carga calculada para la subestación en la situación de degradada simple. Para los casos de degradar parcialmente las subestaciones 4 y 7 , no habría ningún problema como se ve en la gráfica. En cambio en cuando la subestación 10, cae un transformador, se ve que los picos de intensidad superan lo permitido, por lo que se necesita pensar en una solución para evitar este fallo del sistema. La solución más sencilla es aumentar la potencia de la subestación 10, o bien incluyendo un nuevo grupo transformador, tal y como tienen el resto de subestaciones, o bien aumentando la potencia de los existentes.

\begin{tabular}{lllll}
\hline $\begin{array}{l}\text { Fallo } \\
\text { doble }\end{array}$ & SS1 & SS4 & SS7 & SS10 \\
\hline SS1 & & 963 & 885 & 830 \\
SS2 & 1902 & 1545 & 1340 & 1247 \\
SS3 & 1661 & 2312 & 1632 & 1471 \\
\hline
\end{tabular}




\begin{tabular}{lllll}
\hline $\begin{array}{l}\text { Fallo } \\
\text { doble }\end{array}$ & SS1 & SS4 & SS7 & SS10 \\
\hline SS4 & 1916 & & 2306 & 1870 \\
SS7 & 1822 & 2271 & & 1939 \\
SS8 & 1486 & 1602 & 2157 & 1876 \\
SS9 & 995 & 1051 & 1317 & 1416 \\
SS10 & 1054 & 1081 & 1208 & \\
\hline
\end{tabular}

Tabla 39 Irms a 3600s para los casos estudiados en fallo completo 


\subsubsection{Diseño para mejor valor función objetivo Costes de Operación}

La configuración de este segundo ejemplo se ha escogido para mostrar porque, al pesar tan poco las subestaciones A-F, el algoritmo podía eliminar una y no causar un gran cambio en el valor total fitness1. Por lo que los algoritmos han encontrado otro diseño de las subestaciones con la siguiente propuesta:

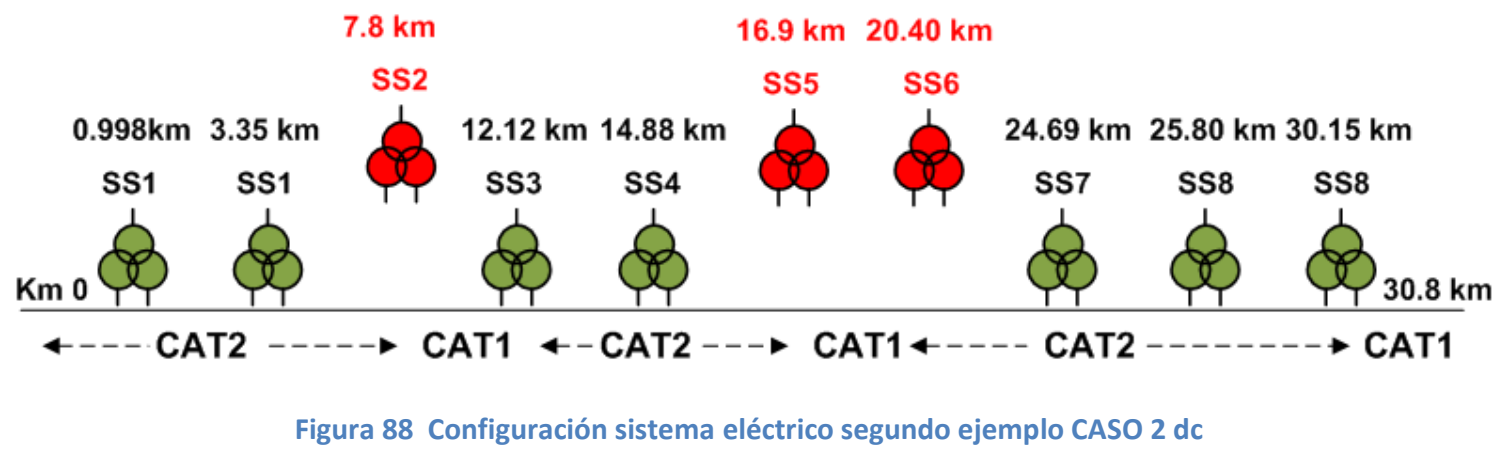

A diferencia de la anterior propuesta, esta elimina una subestación más, pero también añade mucho más cable para feeder de retorno, seguramente para compensar la subida que se va a producir en las tensiones de retorno, debido a la subestación que ya no está. Como se ha examinado antes, no parece que la línea como estaba diseñada inicialmente tuviera problemas de caídas de tensión, por lo que los mayores problemas al ajustar el dimensionamiento, van a aparecer en posibles sobrecargas en subestaciones o en tensiones de retorno demasiado elevadas, por encima de los $170 \mathrm{~V}$ permitido en tensiones instantáneas.

Siguiendo el procedimiento ya habitual para el lector, se van a mostrar los resultados eléctricos más significativos para situaciones normal y degradada, para posteriormente comentar el impacto de los datos más relevantes.

\section{- Funcionamiento normal}
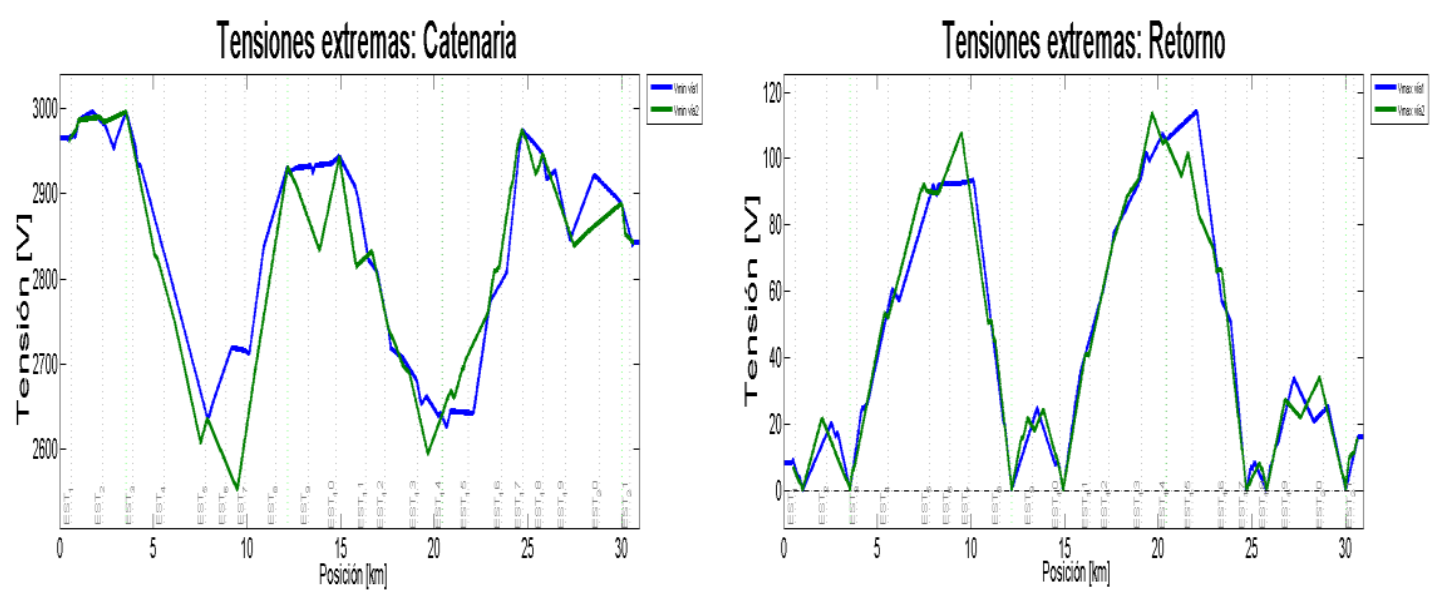

Figura 89 Ejemplo 1 Funcionamiento normal. a) tensiones mínimas catenaria;b) tensiones máximas retorno

Observando las gráficas de tensiones extremas, se confirma que los espacios eléctricos liberados por las subestaciones eliminadas, son los que se han de examinar 
con más detenimiento. Es muy probable que si bien no haya problemas con la caída de tensión, para la tensión de retorno se haya de volver a calcular las tensiones instantáneas en puntos kilométricos donde se prevé, vayan a superarse los $120 \mathrm{~V}$.

Como la situación en las cabeceras de la línea no ha variado mucho, debido probablemente a la falta de respuesta del dimensionamiento si se hubiera eliminado alguna de las cabeceras o adyacentes, no se va a volver a probar la incidencia de las situaciones degradadas en terminales. Por el contrario se van a estudiar las adyacentes a las zonas donde se produce, según se observa en las gráficas antes mostradas, las caídas de tensión mayores, es decir se va a trabajar con las SS2, SS4, SS5 y SS8

\section{- Situaciones degradada simple, o caída de un grupo en la subestación}
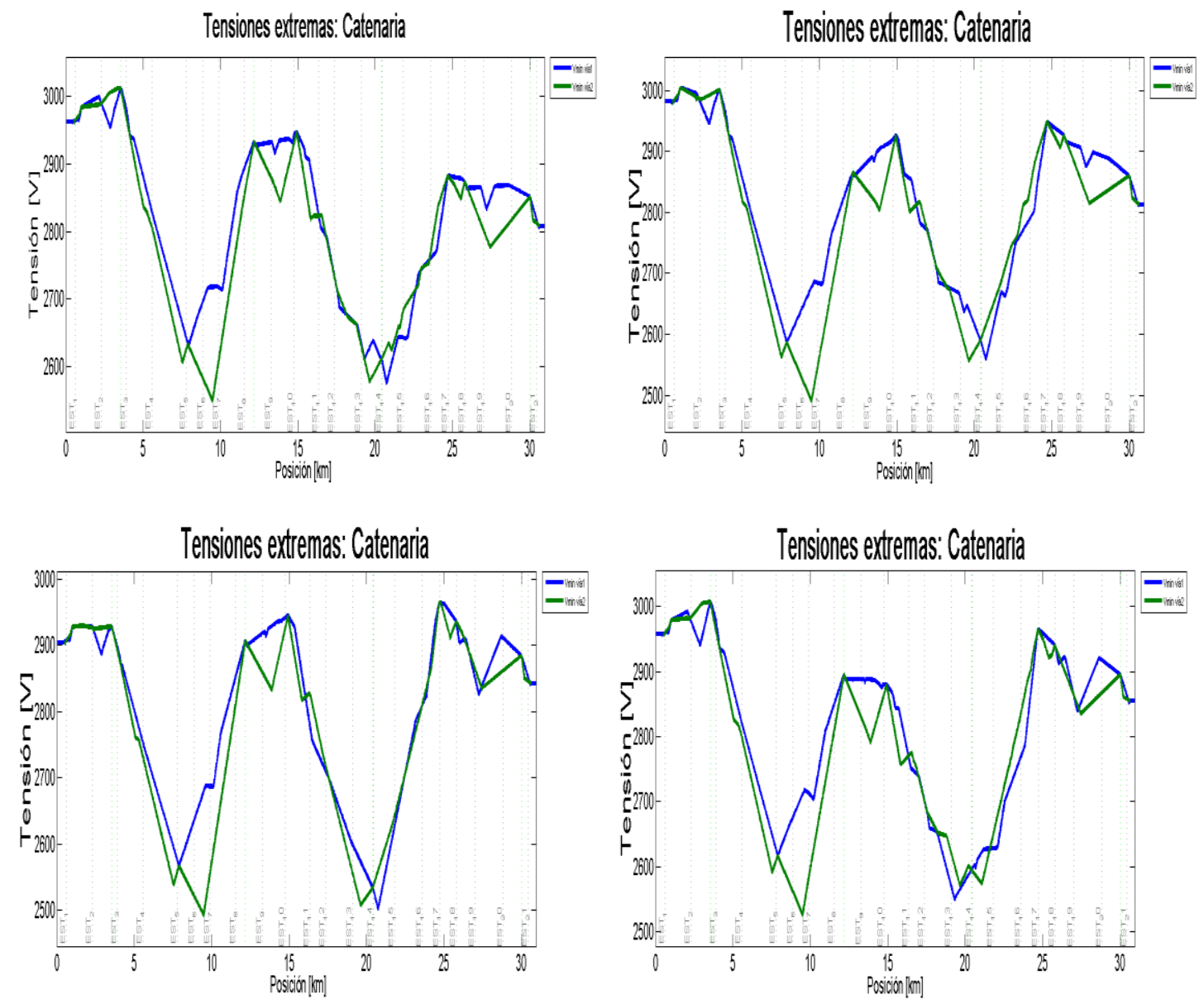

Figura 90 Tensiones mínimas catenaria. a) fallo simple ss5; b) fallo simple ss4; c) fallo simple ss10; d) fallo simple ss2

La tensión mínima se sitúa alrededor de los $2600 \mathrm{~V}$ para el caso b), muy lejos del mínimo permitido de $2100 \mathrm{~V}$. 

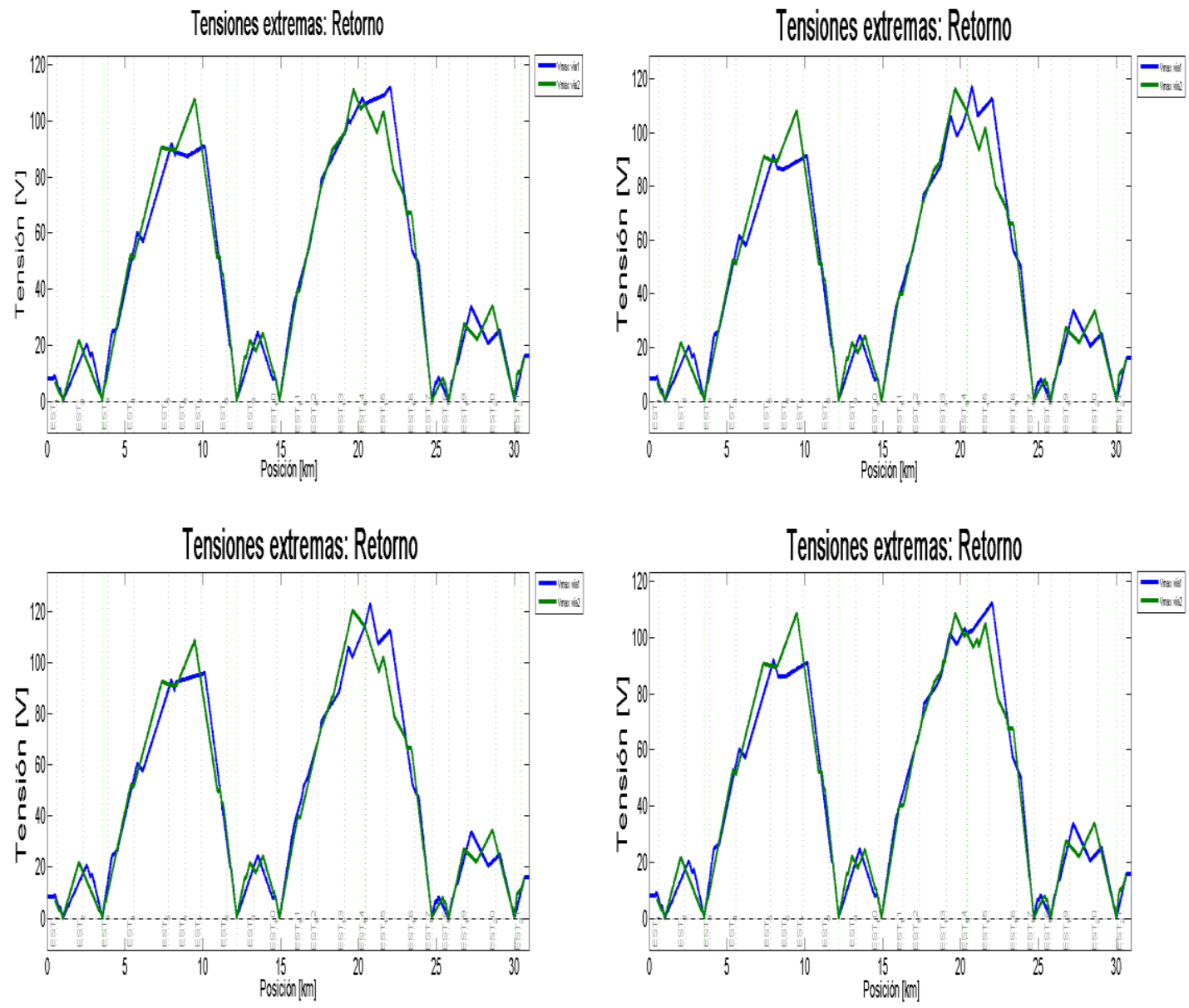

Figura 91 Tensiones de retorno para los tres casos mencionados anteriormente

En esta ocasión si que se superan los valores de $120 \mathrm{~V}$, por lo que ha hecho falta el estudio del régimen permanente de 5 minutos, para comprobar la tensión en este intervalo de tiempo. En todos los casos, ese resultado no superaba los $80 \mathrm{~V}$, por lo que no se prevé un problema para el sistema, las tensiones de retorno en operación. 


\section{- Situaciones degradadas doble o fuera de servicio una subestación}

Las situaciones críticas examinadas son las mismas que para el fallo simple. Para esta parte se ha añadido el caso de la otra subestación terminal.
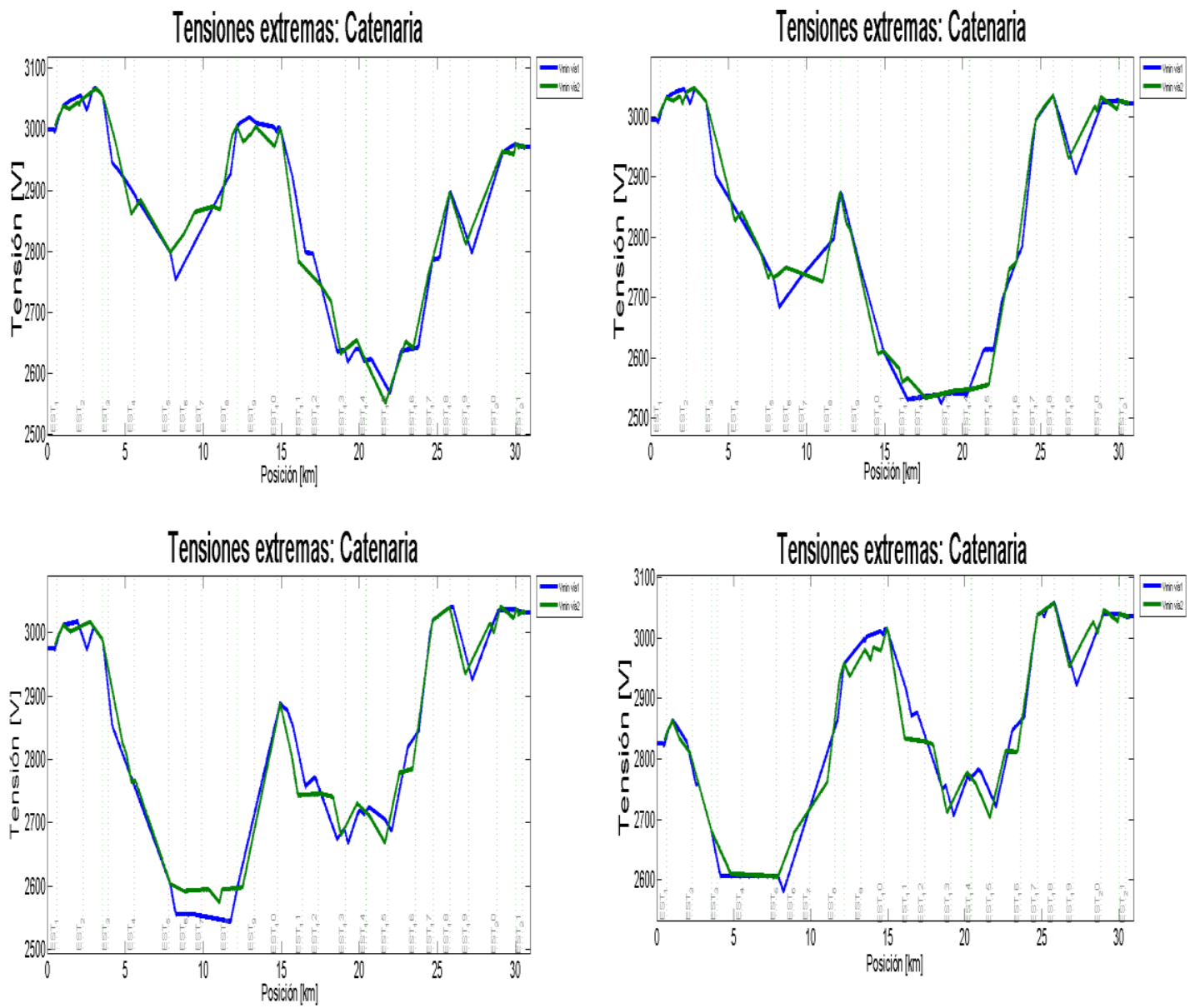

Figura 92 Fallo completo subestación. a) ss8; b) ss5;c) ss4; d) ss2 

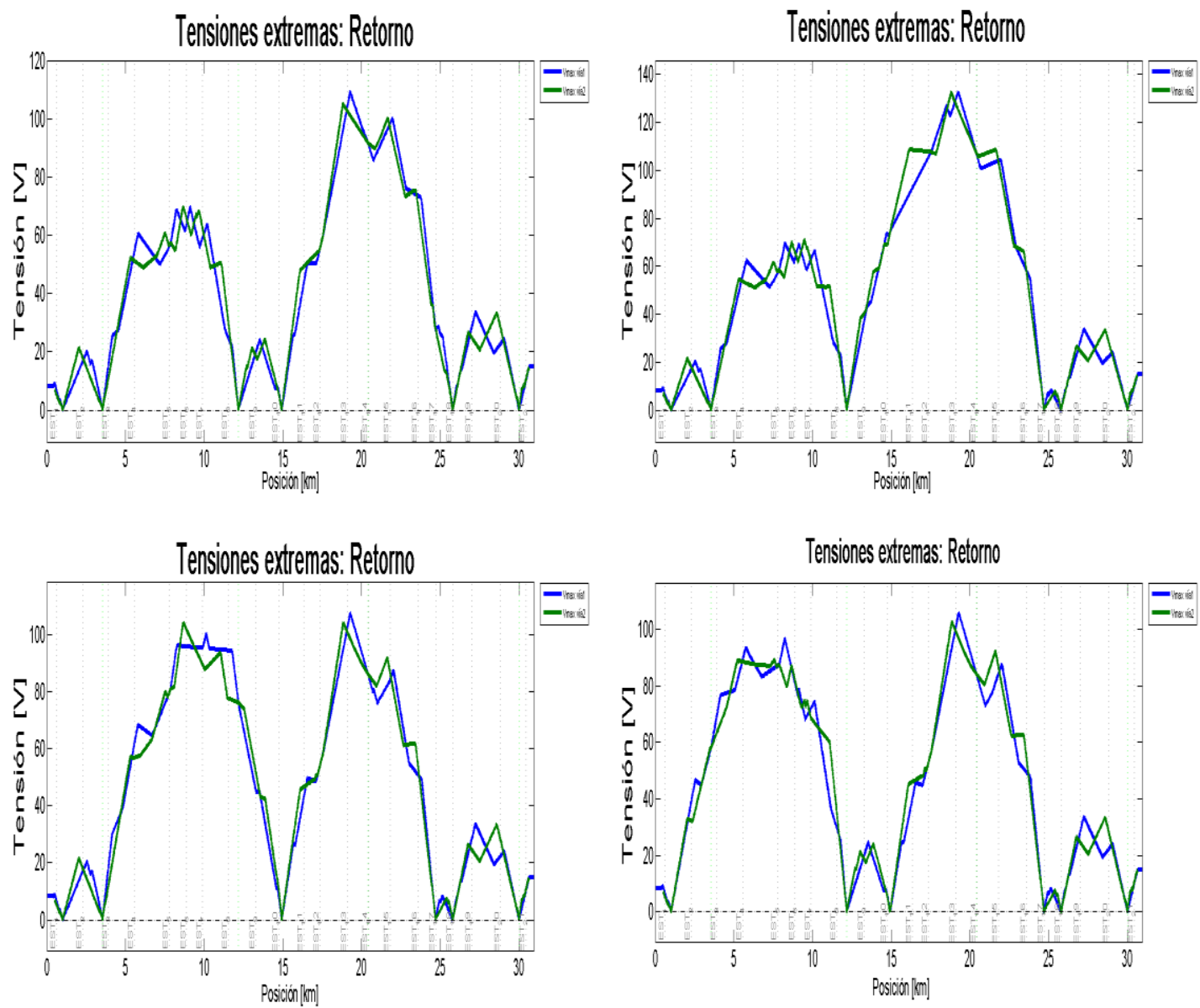

Figura 93 Tensiones máximas de retorno para los casos elegidos

Por último se presentan los valores de intensidades medias cuadráticas, que permiten evaluar si una subestación puede llegar a tener problemas de sobrecarga.

\begin{tabular}{llllll}
\hline $\begin{array}{l}\text { Fallo } \\
\text { simple }\end{array}$ & Normal & SS2 & SS4 & SS5 & SS8 \\
\hline SS1 & 1753 & 1855 & 1624 & 1596 & 1573 \\
SS2 & 2610 & 2176 & 2729 & 2664 & 2610 \\
SS4 & 2921 & 3033 & 2372 & 3219 & 2953 \\
SS5 & 2947 & 2995 & 3233 & 2406 & 3027 \\
SS8 & 2743 & 2751 & 2784 & 2833 & 2206 \\
SS9 & 1807 & 1811 & 1828 & 1850 & 2084 \\
SS10 & 1763 & 1766 & 1777 & 1785 & 1905 \\
\hline
\end{tabular}

Tabla 40 Irms a 3600s para los casos estudiados en fallo simple

En naranja se han destacado las situaciones que estarían por encima de la intensidad nominal de las subestaciones, 1000 y 2000 A respectivamente. En esta situación hay que estudiar el ciclo de carga para conocer si existe o no sobrecarga.

Se calculan las curvas de carga, cuyo resultado se muestra a continuación: 

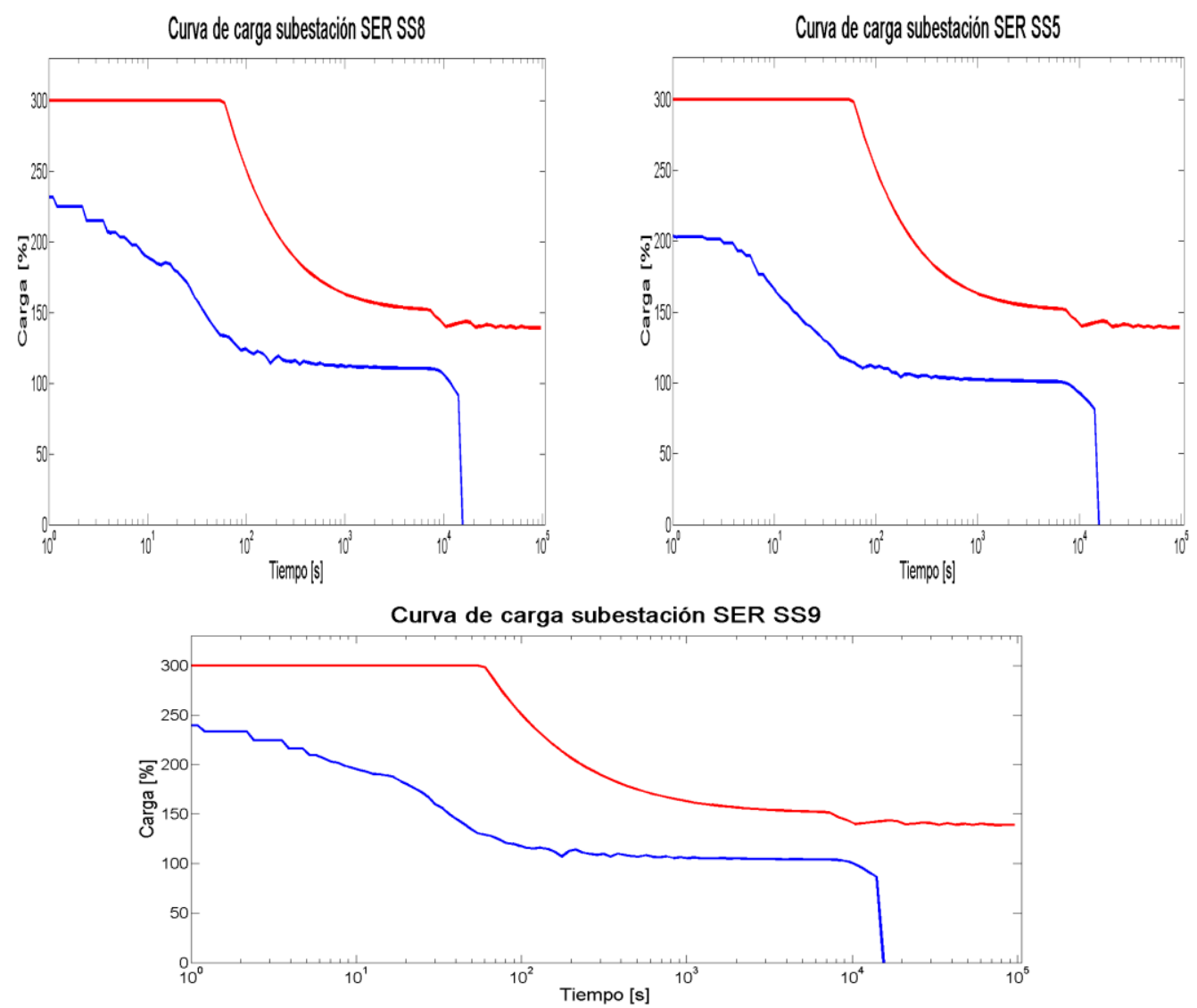

Figura 94 Curvas de sobrecarga para situación de estudio SS8
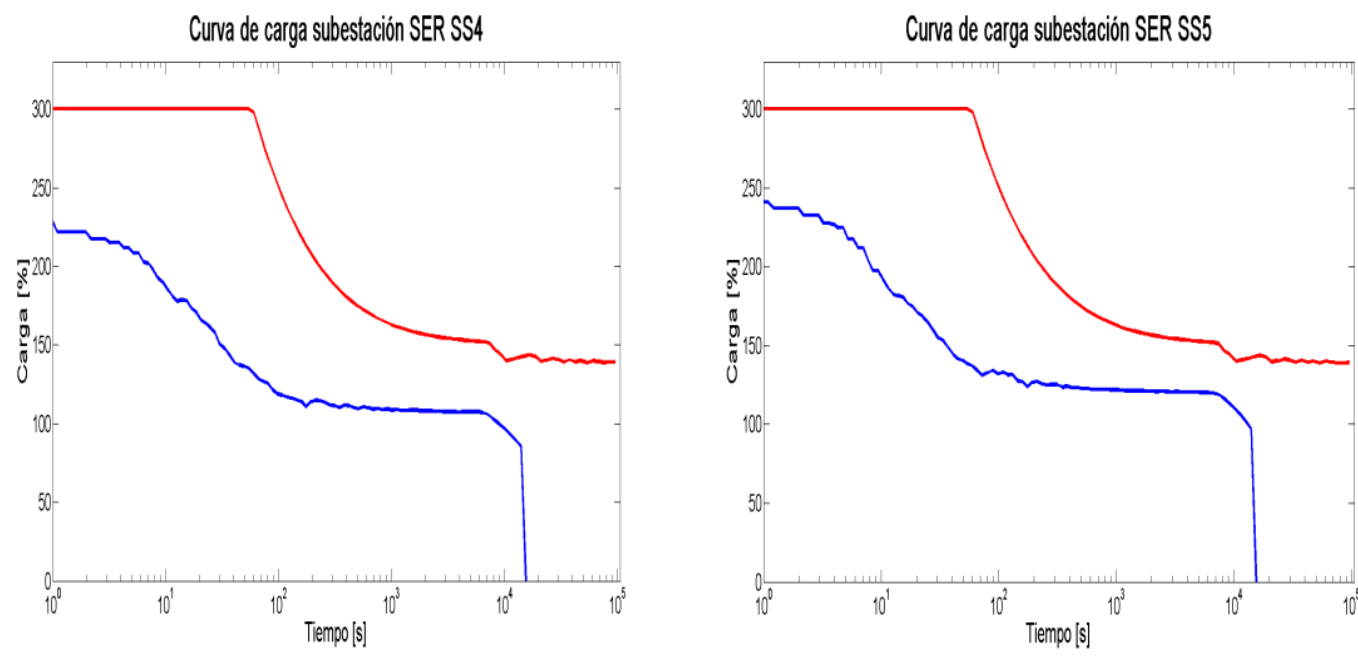

Figura 95 Curvas de sobrecarga para situación de estudio SS5 

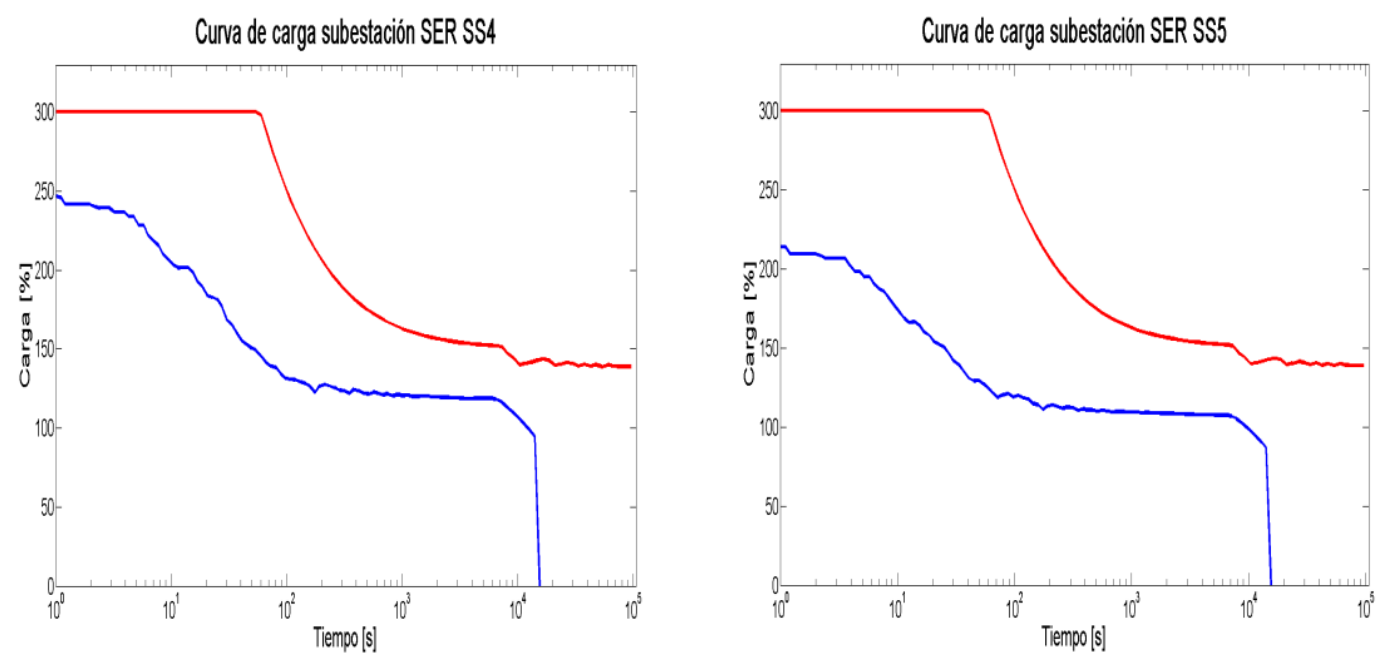

Figura 96 Curvas de sobrecarga para situación de SS4
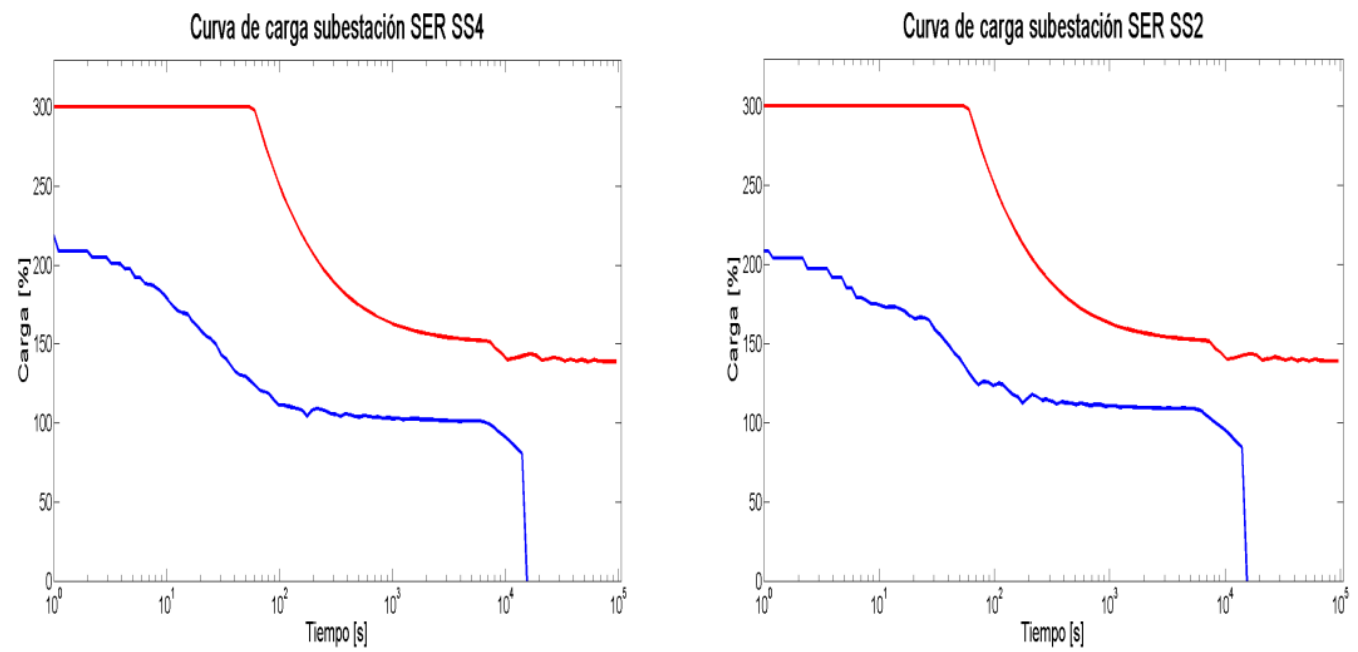

Figura 97 Curvas de sobrecarga para situación de SS2

En este caso, no se aprecia para ninguna de las situaciones límite analizadas con los valores rms de las intensidades, que sobrepase la carga permitida para la Clase de material rodante elegida.

\begin{tabular}{lllll}
\hline $\begin{array}{l}\text { Fallo } \\
\text { doble }\end{array}$ & SS2 & SS4 & SS5 & SS8 \\
\hline SS1 & 2100 & 1200 & 1224 & 1238 \\
SS2 & & 2152 & 1922 & 1745 \\
SS4 & 2340 & & 2991 & 2017 \\
SS5 & 2138 & 2978 & & 2238 \\
SS8 & 1909 & 2024 & 2229 & \\
SS9 & 1196 & 1253 & 1351 & 2139 \\
SS10 & 1149 & 1178 & 1107 & 1138 \\
\hline
\end{tabular}

Tabla 41 Irms a 3600s para los casos estudiados en fallo doble 


\subsection{Comparación y conclusiones}

Para sistemas de corriente continua la metodología se ha mostrado bastante útil atendiendo a los resultados de los ensayos realizados. La adecuación formal de los parámetros relativos a la manipulación del comportamiento de los algoritmos genéticos, sobre todo los propios de la Metodología, parámetros de coste y discretización zonal, han permitido conocer hasta qué punto influyen en la diversidad y homogeneidad de los resultados finales. Para el Caso 2 se ha elegido un ejemplo de cómo afectaría la optimización a un proyecto de sistema eléctrico, donde algunos de los principales criterios se manejaban con márgenes muy escasos de variación. Los Frentes de Pareto óptimos finales van en consonancia con esta característica de rigidez en algunos componentes del sistema eléctrico, y reflejan tal y como se esperaba cierto grado de homogeneidad en los resultados finales. En todo caso el diseñador se encontraría con conjuntos de soluciones que superan las prestaciones de los restantes posibles escenarios. Acudiendo a otro baremo para clasificar los algoritmos según los resultados, se tiene los tiempos de cálculo empleados por cada uno de ellos. En las tablas aparece claramente destacado el algoritmo NSGA-II como el que más tiempo ocupa, sin que se obtenga un beneficio claro respecto de las otras dos, por lo que se puede concluir que para Proyectos donde existan condiciones iniciales muy estrictas, sería más aconsejable utilizar las técnicas AMGA-II y épsilon-MOEA antes que la NSGA-II.

Sobre los análisis eléctricos de validación de los sistemas propuestos por la Metodología, se ha mostrado bastante eficiente en la solución final, y aportando siempre mejoras en costes tanto presupuestario como de adecuación a un dimensionamiento más acorde con las necesidades de potencia del sistema. En los dos casos elegidos, las situaciones degradadas estudiadas cumplen las restricciones marcadas por la normativa, salvo en un caso de degradada completa, que supera por un pequeño margen el límite de sobrecarga. Esto se solventaría añadiendo más potencia a la subestación adyacente, liberando de carga a la que sufre este régimen de sobrecarga. 


\section{Bibliografía}

[1] W. D. Johnston, «Mainline railway electrification, an economic feasibility model. Ph. D Thesis,» Commerce and Business administration, University of British Columbia, 1975.

[2] C. H. Bae, «A simulation study of installation locations and capacity of regenerative absorption inverters in DC 1500 V electric railways system," Simulation Modelling Practice and Theory, vol. 17, no 5, pp. 829-838, 2009.

[3] T. Capuder, L. Lugaric, J. Brekalo-Strbic y S. Krajcar, «Optimizing the train power system in Zagreb,» de Vehicle Power and Propulsion Conference VPPC'O9 IEEE, 2009.

[4] F. González y E. Manzanedo, «Optimal Design of a D.C. Railway Power Supply System,» de IEEE Electrical Power \& Energy Conference, 2008.

[5] H. J. Chuang, K. Yuan y Lu-Chu, «Optimization of inverter placement for mass rapid transit systems by immune algorithm,» IEEE Proceeding Electric Power Applications, vol. 152, pp. 61-71, 2005.

[6] J. Doyne-Farmer y H. P. Norman, «The immune system, adaptation, and machine learning," Physica D: Nonlinear Phenomena, vol. 22, no 1-3, pp. 187-204, 1986.

[7] F. Henrique-Pereira, C. Lobo-Pires y I. Ikuyo-Nabeta, «Optimal placement of rectifiers substation on DC traction systems, "IET Electrical System in Transportation, vol. 4, no 3, pp. 62-69, 2013.

[8] M. Wall, GAlib: A C++ library of genetic algorithm components, Massachusetts: Mechanical Engineering Department. MIT, 1996.

[9] N. H. Viet, H. S. Song y K. Nam, «Locating power supplies on a Personal Rapid Transit System to minimize system losses, 》 IEEE Transactions on Industry Applications, vol. 40, no 6, pp. 1671-1677, 2004.

[10] C. Chang, J. Low y D. Srinivasan, «Application of tabu search in optimal design and operation of MRT power supply systems,» IEEE Proc. Electric Power, vol. 146, pp. 75-80, 1999.

[11] F. Glover, «Tabu search: A tutorial interfaces,» vol. 20, no 4, pp. 74-94, 1990.

[12] C. Chang, A. L. Wang, F. Wen y D. Srinivasan, «Genetic algorithm based bicriterion optimization for traction substation in dc railway systems, 》 de IEEE International Conference on Evolutionary Computation, 1995.

[13] M. Olofsson, G. Andersson y L. Söder, «Optimal operation of the Swedish railway electrical system,» de International Conference on Electric Railways in a United Europe, 1995. 
[14] T. Kneschke, "Simple Method for Determination of Substation Spacing for AC and DC electrification systems, "I IEEE Transactions on industry applications, Vols. \%1 de \%2IA-22, no 20, pp. 763-780, 1986.

[15] C. Hui-Jen, L. Chu, C. S. Chen, C. H. Lin y J. Y. Chen, «Optimal Expansion Planning of Traction Substations for an Electrified Mass Rapid Transit System,» de International Conference on Power System Technology, Chongquing, 2006.

[16] Y. C. Liu y J. F. Chen, «Control scheme for reducing rail potential and stray current in MRT systems, 》 IEEE Proceedings electric power applications, vol. 152, no 3, pp. 612-618, 2005.

[17] C. Schifers y G. Hans, «IEEE standard for communications based train control (CBTC) performance and functional requirements, " de Vehicular Technology Conference Proceedings, VTC 1581-1585, 2002. 


\section{Aplicación de la Metodología en Líneas Ferroviarias de AC. Pruebas y Resultados.}

Este segundo apartado de Aplicación está dedicado a los sistemas ferroviarios que tienen sistemas de electrificación en corriente alterna. Al igual que para los sistemas explicados en el capítulo anterior, tras la implantación de los sistemas informáticos, se ha abierto una puerta para la aplicación de técnicas aplicadas, que combinadas con el conocimiento de las características del dimensionamiento eléctrico en $\mathrm{AC}$, han hecho que diversos investigadores creyeran interesante aportar metodologías para mejorar las prestaciones o minimizar costes del sistema.

Tras una explicación del sistema en general, estado del arte y adaptación al sistema experto, se explicarán las características principales de las líneas escogidas para las pruebas y un breve análisis del dimensionamiento de cada configuración inicial. Finalmente se mostrarán y detallarán el proceso de optimización, los resultados finales dependiendo de parámetros y algoritmo genético usado y por supuesto, un breve análisis del dimensionamiento resultante para cada caso.

\subsection{Introducción y Estado del Arte.}

Las investigaciones y trabajos que tienen como sistema eléctrico en alterna, ya sea monotensión o bitensión, tienen como fundamentos principales los explicados en el Capítulo 3. En el Capítulo 5 se ha conectado la abstracción del modelo eléctrico a la metodología elegida. Estas son las bases en las que se suele cimentar los estudios, por lo que a continuación se pasará a relatar el estado del arte en investigaciones cercanas o que han sido de interés para progresar en nuestras investigaciones y desarrollo del sistema.

\subsubsection{Sistemas de corriente alterna}

La tarea de desarrollar el método para corriente alterna ha sido gradual. Como primer enfoque se trató de aplicar a sistemas de 1x25, conocidos como monotensión. Con este propósito se obtuvieron resultados que se presentarán en el CASO 1. En este caso se aplica el algoritmo genético NSGA-II.

La siguiente etapa debía comprender ya la inclusión del sistema dual/bitensión ó $2 \times 25$. Para la correcta aplicación se debía buscar un sistema de alta velocidad y por supuesto, comprobar el funcionamiento instalando autotransformadores y zonas neutras. En definitiva, se ha escogido un caso real para el estudio completo de la casuística de este tipo de sistemas.

\subsubsection{Estado del arte}

Las estrategias para mejorar el dimensionamiento eléctrico en $\mathrm{AC}$, debido a que la complejidad es mayor que para los sistemas de corriente continua, deben atenerse a más condicionantes y por tanto, los sistemas de optimización serán más complejos. Por 
supuesto, la posibilidad de sistemas en modo simple (1x25) y dual (2x25), añaden tanto más complejidad como mayor extensión en las áreas de estudio, que en el sistema anterior en corriente continua. Debido a esto, los procesos de mejora estudiados hasta el momento, todos tratan de conseguir un avance sustancial, teniendo en cuenta áreas específicas del sistema. Las consecuencias que tiene la instalación de sistemas de alta tensión a lo largo de la línea, también deben ser analizadas y ver qué implicaciones tienen sobre su entorno. Y finalmente, los costes presupuestarios y de impacto, también son diferentes a los que se tratan en sistemas, normalmente de menos envergadura, que los tratados en el Capítulo 5.

El relato de los distintos trabajos asociados a este tipo de sistemas eléctricos, se va a realizar de forma que se transmita lo más relevante de las investigaciones referidas a esta temática. La diferenciación no va a tener en cuenta el tipo de sistema, es decir si es monotensión o dual, si en cambio va a primar la relevancia de estudios y ámbito de aplicación. El trabajo presentado por Batistelli y otros [1] establece un modelo para estudiar las condiciones básicas de un sistema eléctrico en $2 \times 25$. Este modelo sirve tanto para observar las conclusiones aportadas por las pruebas realizadas por los autores, como para dar un primer enfoque interesante para el trabajo de pruebas con sistemas en alterna $2 \times 25$.

El trabajo realizado por Knestchke [2], del que ya se comentó en el Capítulo anterior, puede definir claramente lo expuesto acerca de la complejidad de uno y otro tipo de sistemas. El trabajo se realizó pensando sobre todo en adaptarlo a sistemas de corriente alterna, pero la estructura del trabajo permitía a su vez, adaptarlo también a sistemas de corriente continua, como se puede leer la referencia en el Capítulo 5. El autor además de la zonificación que hace respecto de la situación de las subestaciones y material rodante, aplica el mismo modelo para obtener el número y posición de autotransformadores de la línea, por lo que el mallado eléctrico contempla esta etapa de mayor nivel. Con el estudio puede llegar a conclusiones acerca del espaciamiento entre autotransformadores en la línea ferroviaria que esté siendo comprobada. El análisis e impacto de los armónicos de las corrientes producidas por el sistema de AC, también es tenido en cuenta por el autor.

De entre los trabajos más destacados están los realizados por E. Pilo. Ya se había hablado del grupo de trabajo ya que uno de los principales pilares es el desarrollo del simulador Silvia. La mayor parte de la investigación de optimización del diseño, la han realizado en sistemas de corriente alterna. Uno de los primeros avances, fue la simplificación realizada de sistemas de 2x25 [120], que también aparece en su Tesis [4]. Lógicamente debe tener en cuenta para la simplificación tanto la catenaria como la acción de los autotransformadores, la principal diferencia respecto al sistema mono. El trabajo asume diversas suposiciones. Entre ellas que las caídas de tensión en las celdas tienen el mismo valor en catenaria y feeder negativo, pero con distinto signo, y la segunda es que los autotransformadores se suponen ideales. Solo los autotransformadores que estén en las celdas adyacentes al tren tienen flujo de corriente. 
La aplicación de la simplificación es inmediata para la optimización en sistemas ferroviarios de alta velocidad, y que en España, suelen trabajar en sistema 2x25 ó mixtos. Por partes el trabajo examina cuál debería ser la óptima relación entre repositorio de catenarias posible en la línea, respecto al impacto de aumentar/disminuir el número de autotransformadores [5]. Para finalmente concluir con el trabajo [6], en el cual se combinan todos los anteriores para calcular el mejor diseño para sistemas de alta velocidad funcionando en $2 \times 25$. Tratan de buscar el número y posición adecuados para subestaciones, autotransformadores y zonas neutras. Para ello usan la técnica MIP (Mixed Integer Programming). Otra aproximación al problema, acogiendo la mayor parte de investigaciones de su predecesor es la que se puede leer en [7]. El autor realiza un cambio en el método de optimización para trabajar con un algoritmo genético. Esta vía de investigación permitió el planteamiento de diversas cuestiones para aplicar en nuestra Tesis, como la afectación de nuevos pesos para calcular las funciones objetivo (zonificación), planteamiento de análisis de nuevas restricciones (presupuesto), o integración de algoritmos genéticos más novedosos y compararlos para obtener una nueva métrica de utilización de los mismos en trabajos de Ingeniería.

Analizar el sistema eléctrico de tracción para ver cómo se puede mejorar a partir de los resultados, es una de las aportaciones más comunes que se han realizado hasta el momento, en cuestión de optimización de este tipo de sistemas. El análisis de los sistemas $2 \times 25 \mathrm{kV}$, tiene en el trabajo [8] uno de los precursores. De modo que al modelar a una escala pequeña un sistema eléctrico dual en alterna, permite a los usuarios una herramienta de análisis sencilla de entender y lo más importante, de aplicar tanto directamente como en simuladores integrados. Otro modelo de sistema en alterna, que en definitiva tiene la misma idea final, es la representada por [9]. La mayor novedad de este trabajo es que los módulos eléctricos están implementados en Matlab/Simulink. Esta herramienta está orientada sobre todo para la mejora de la estabilidad del sistema, ya que han integrado distintos fallos que suelen presentarse en este tipo de sistemas. Como precursor de este tipo de simuladores [10], cuyo objetivo es conseguir una herramienta lo suficientemente potente para que al analizar los resultados, esto permita que se pueda optimizar la alimentación de energía para la tracción y mejorar la regulación de la tensión en catenaria. Otros trabajos están dirigidos a mitigar los posibles efectos de afectaciones o distorsiones que se producen en sistemas monofásicos. En [11], para sistemas 1x25, tratan de mejorar la calidad de la potencia emitida por los generadores. Con filtros de la potencia activa inyectada en la red y controlada por un sistema con histéresis integrada, pretenden rebajar la posible distorsión de los armónicos en los cables conductores.

El efecto de los armónicos en los sistemas eléctricos puede provocar el fallo total de los dispositivos. La aparición de este efecto en los sistemas de los motores de los trenes adquiere cierta importancia, por lo que el control de los armónicos suele ser uno de los factores a tener en cuenta cuando se está diseñando un sistema eléctrico de tracción. Los autores Lee y otros [12], buscan a partir de simulaciones y de datos obtenidos de sistemas reales en ferrocarriles de Corea, hallar la mejor localización para los bancos de 
rectificadores (Rc-Bank). Este sistema lo han aplicado a sistemas 2x25, líneas de alta velocidad.

A partir de los trabajos de modelar y simular el comportamiento de los sistemas de corriente alterna, se puede observar un aumento de la capacidad de analizar otros aspectos más concretos del dimensionamiento. Un ejemplo se encuentra en [13], donde los autores en base al cálculo y comportamiento de los flujos de carga de las pruebas realizadas con simuladores, pretenden llegar a conclusiones acerca de la estabilidad del dimensionamiento tal y como está configurado en el momento de realizar los tests. Otro de los problemas importantes que se han de acometer a la hora de realizar estudios de este tipo, es la influencia de la tensión en la catenaria entre sectores dispuestos por autotransformadores. El trabajo realizado por [14] está enmarcado y dirigido a sistemas de alta velocidad.

Los trabajos puramente analíticos conforme ha ido evolucionando las tecnologías de la información, han ido perdiendo peso. Al igual que en los estudios con corriente continua, los investigadores han ido incorporando nuevas técnicas de optimización aplicada. Una de estas técnicas, son las Redes Neuronales [15]. El aprendizaje del comportamiento de un sistema determinado, que es la principal característica de las Redes Neuronales, fue aprovechado para aprender acerca de las respuestas de un sistema eléctrico [16]. El estudio se sitúa en el sistema eléctrico sueco, que funciona a $15 \mathrm{kV}$ y $16.7 \mathrm{~Hz}$. El aprendizaje tiene en cuenta el tráfico, la velocidad máxima de los trenes y los costes de los principales componentes involucrados. El segundo paso de la metodología, cuando ya la Red es capaz de prever cuál sería el resultado de un sistema determinado, se basaría en evaluar los distintos escenarios que se propongan como solución del dimensionamiento. Los autores tienen diversos trabajos de interés en este campo y en el estudio del dimensionamiento eléctrico, tanto en continua, como sobre todo en alterna. En [134], estudian el impacto de los flujos de carga sobre el diseño del dimensionamiento eléctrico. Otro interesante aproximación la realizan con la creación de un modelo de optimización de los flujos de carga (OPF), aplicando una técnica de optimización de programación mixta (MINLP). Este trabajo está dirigido [18] a sistemas de alta velocidad.

En general se puede convenir que el cómputo general de investigaciones relacionadas con el tema principal de esta Tesis no es muy elevado. Las causas pueden ser debidas al complejo modelo global que se ha de construir para llevar a cabo este tipo de optimizaciones. Los trabajos elegidos como más representativos en el campo de mejoras en sistemas eléctricos de tracción en corriente alterna, se suelen focalizar en aspectos muy concretos, como pueden ser la eliminación de armónicos o el impacto de los flujos de carga en el sistema eléctrico de tracción. Los trabajos relacionados fuertemente con esta Tesis, han aportado un importante punto de partida para que desde este trabajo se pueda intentar aportar otros enfoques para encarar el problema, como también tratar de mejorar en algunos aspectos como puede ser la introducción de diversos algoritmos genéticos, aplicación de un modelo de simulación distintos, o 
permitir análisis de otro tipo de cumplimiento de restricciones. Todo esto se va a presentar en los dos ejemplos que aparecen en los apartados siguientes. 


\subsection{Líneas ferroviarias en AC para optimizar. Configuración inicial y Resultados.}

El CASO 1 aplicado a la Metodología, pertenece una línea ferroviaria con datos creados expresamente para las pruebas de la primera fase de la corriente alterna monotensión. En este sentido, se preparó una línea con un sobredimensionamiento eléctrico y que además, presentaba una fuerte pendiente en la mitad del recorrido. Al ser una línea no-real permite la inclusión de flexibilidad absoluta a la hora de trabajar con la discretización zonal y la ponderación de los elementos involucrados. Esto último proporciona un conjunto de posibles pruebas para comprobar el comportamiento de la Metodología.

Para el CASO 2, y tratándose de pruebas para el caso dual (2x25), si que se configuró y trabajó con un sistema real. El sistema ferroviario escogido es muy interesante, desde el punto de vista de validar un dimensionamiento eléctrico, ya que contenía en las especificaciones propias del proyecto una parte fija de 1x25 y el resto de $2 \times 25$, lógicamente separadas ambas por zona neutra. Además el proyecto contenía varias zonas de muy baja permisividad para la instalación de componentes. Esto hace que las pruebas hayan permitido examinar diversas situaciones de trabajo de la Metodología, y la complejidad del estudio también ha sido elevada, incrementando así el examen de validez de las conclusiones halladas. 


\subsection{CASO 1}

\subsubsection{Infraestructura en la línea}

La línea tiene una longitud de $60 \mathrm{~km}$ y se han establecido 3 estaciones. Incluye limitaciones de velocidad distribuidas a lo largo de la línea. El diseño del trazado incorpora elevados gradientes para el alzado, por lo que el tren deberá intentar rebasar las dificultades que imponen las rampas y pendientes. La limitación de velocidad en la línea se ha determinado muy alta, $350 \mathrm{~km} \cdot / \mathrm{h}$.

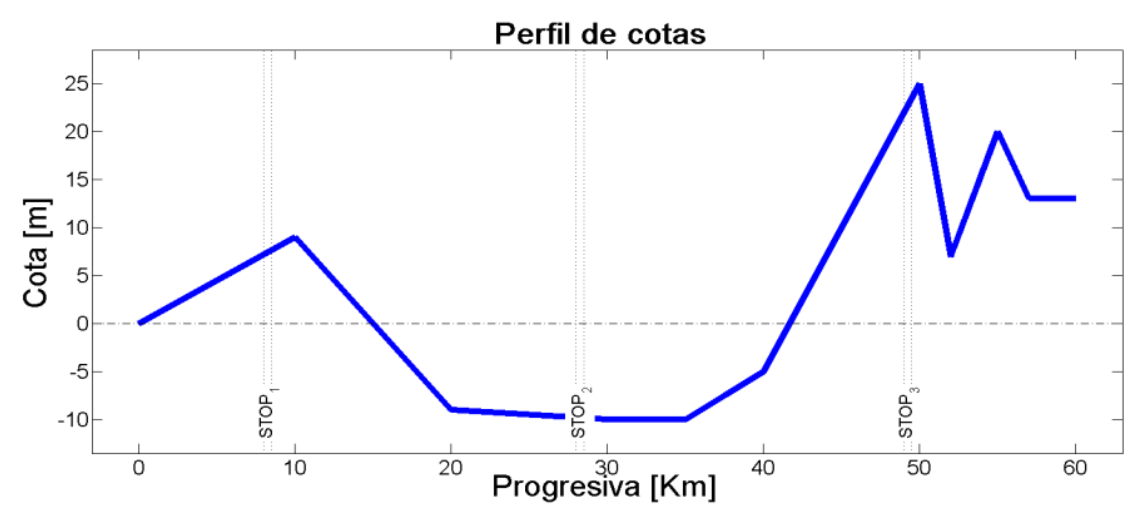

Figura 98 Perfil de alzado de línea para CASO 1

\subsubsection{Material rodante}

Configuración de la distribución de los coches del tren:

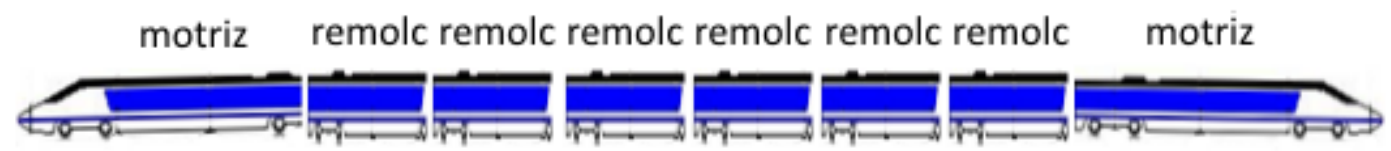

Figura 99 Ejemplo ilustrativo distribución coches en tren

M-R-R-R-R-R-R-M $\rightarrow$ Dos coches motrices cabeceros y los 6 restantes motrices remolcados.

\begin{tabular}{c|c}
\hline Propiedades & Valores \\
\hline Masa & $485 \mathrm{Tm}$ \\
Masas Giratorias & $48 \mathrm{Tm}$ \\
Pasajeros & 500 \\
Coeficientes resistencia avance & $\mathrm{A}[\boldsymbol{N}]=\mathbf{3 5 7 0}$ \\
& $\mathrm{B}[\boldsymbol{N} * \boldsymbol{s} / \boldsymbol{m}]=\mathbf{1 2 0 . 3 4 4}$ \\
Resistencia en curva (Kc) & $\mathrm{C}\left[\boldsymbol{N} * \boldsymbol{s}^{\mathbf{2}} / \mathbf{m}^{\mathbf{2}}\right]=7.7295$ \\
Deceleración emergencia & 500 \\
Deceleración servicio & $0.8 \mathbf{m} / \boldsymbol{s}^{\mathbf{2}}$ \\
Aceleración tracción & $0.56^{\mathbf{m}} / \boldsymbol{s}^{\mathbf{2}}$ \\
\hline
\end{tabular}




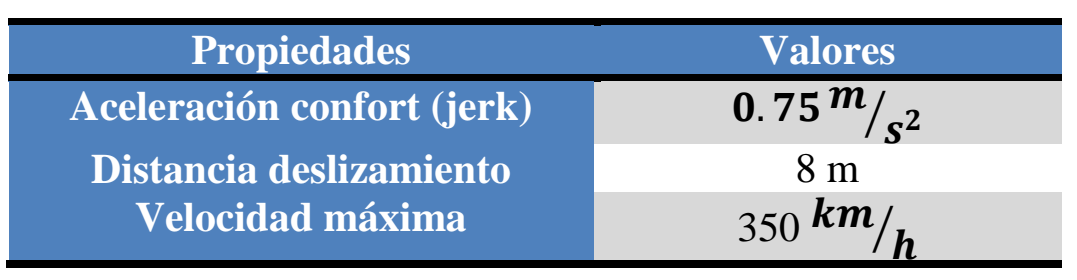

Tabla 42 Propiedades material rodante CASO 1

En la Tabla 1 se han escogido los parámetros más relevantes para conseguir un comportamiento fiel del material rodante en las simulaciones. Tal y como se explicó en Capítulo 3, el procedimiento habitual incluye un conjunto de parámetros de configuración del tren, como son las curvas de tracción y frenado entre otros. Los datos del material rodante si han respetado los utilizados para otra línea ferroviaria, en la que se utilizó el tren con estas características básicas.

\subsubsection{Señalización}

El sistema de señalización que se ha implantado en las simulaciones es el ERTMS2 [19]. El sistema de señalización permitirá seguridad en las circulaciones y también dotará al sistema de un eficaz proceso para calcular la distancia efectiva libre que tienen los trenes por delante. Al igual que para los casos relatados en el Capítulo 5, esta distancia aporta al sistema la información necesaria para calcular la curva de frenado del tren, y por tanto, la velocidad máxima que debería llevar. Esta velocidad permitirá conocer la demanda de potencia en cada ciclo.

\subsubsection{Sistema eléctrico inicial}

Las características principales del dimensionamiento eléctrico en la fase inicial son:

- Sistema de electrificación de corriente alterna AC con tensión nominal de 25 $\mathrm{kV}$.

- Tipos de catenaria posibles:

\begin{tabular}{lcccc}
\hline & $\begin{array}{c}\text { Sección[ } \\
\left.\mathrm{mm}^{2}\right]\end{array}$ & Material & Resistividad $[\Omega / K m]$ & Permeabilidad $[\mathrm{H} / \mathrm{m}]$ \\
\hline$O H L_{1}$ & 107 & Cu Ac 107 & 0.01777 & 1.23 \\
$O H L_{2}$ & 150 & Cu Mg & 0.02778 & 1.23 \\
\hline
\end{tabular}

Tabla 43 Tipos de catenaria en CASO 1. Características principales 
En la configuración inicial se contemplaba la instalación de 4 subestaciones de tracción:

\begin{tabular}{|c|c|c|c|}
\hline & $\begin{array}{l}\text { Potencia } \\
\text { [MW] }\end{array}$ & $\begin{array}{l}\text { Resistencia interna de } \\
\text { pérdidas }[i \Omega]\end{array}$ & Reactancia de pérdidas $[\Omega]$ \\
\hline Subestación & $\begin{array}{l}2 \text { grupos de } \\
10\end{array}$ & 0.001 & 51.8 \\
\hline
\end{tabular}

Tabla 44 Características básicas Subestaciones usadas en CASO 1

Las subestaciones de tracción en esta línea de prueba van a tener dos transformadores cada una. Ambos transformadores estarán situados en sectores eléctricos distintos, por lo que es necesario que en la configuración de las subestaciones se planifique la instalación de una zona neutra para separar ambos transformadores y fases. Teniendo en cuenta esta característica de las subestaciones, la configuración del escenario inicial queda de la siguiente manera:

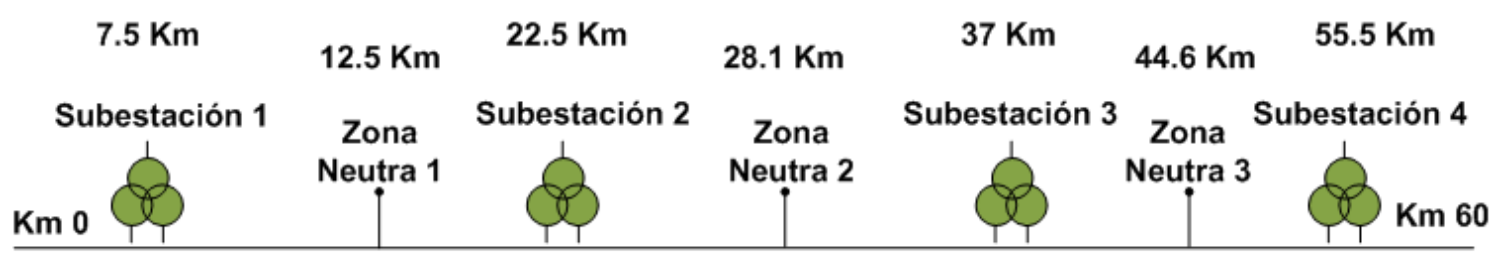

\section{Catenaria Tipo 1}

Figura 100 Configuración elementos sistema eléctrico inicial

\subsubsection{Plan de operación de la línea}

En este caso al no trabajar con un sistema real, se ha tenido que diseñar un plan acorde con las necesidades del estudio. Por una parte no tener ese plan dificulta la tarea inicialmente, pero por otra parte se tiene una gran flexibilidad para alcanzar un plan satisfactorio inicial. Para el plan de explotación se ha tenido que fijar una operación normal de explotación y una degradada, para el caso de fallo de servicio de alguna subestación:

- Escenario normal:

○ Trenes con intervalo de 320s en cada dirección. Tiempos de parada de $50 \mathrm{~s}$ en las paradas STOP1 y STOP3 y de 60s en STOP2.

- Flota de 22 trenes

\section{- Escenario degradado:}

- El intervalo es de 620s. Tiempos de parada en estación similares a situación normal.

- Flota de 14 trenes.

\subsubsection{Configuración algoritmo genético.}

Una de las premisas iniciales de este ejemplo es que los datos del sistema ferroviario son ficticios. Esto provoca que toda la parte implicada en la configuración 
del algoritmo genético también sea ficticia, tanto la ponderación de costes como la discretización zona completa. La idea es que la configuración busque probar ciertos comportamientos previstos de la Metodología. Esto derivaría en análisis específicos de la idoneidad de estos valores y la capacidad del algoritmo.

Para este ejemplo, se usó el algoritmo genético NSGA-II, ya que la implementación del algoritmo se llevó a cabo al mismo tiempo que la configuración de la línea, y posibilitó un trabajo completo para aplicación de la técnica a problemas de monotensión.

\subsubsection{Genotipo}

Según algoritmo explicado en Capítulo 4, por cada subestación de tracción será necesario un gen. Para el sistema 1x25, es necesario la inclusión de zonas neutras, por lo que se añaden 3 genes más, uno por cada posible separación que se pueda llegar a realizar entre las subestaciones de alterna. Para cada posible separación de fase, se plantea también qué tipo de catenaria sería mejor, por lo que también habrán 3 genes más. En total completan el genotipo 10 genes:

\begin{tabular}{|l|l|l|l|l|l|l|l|l|l|}
\hline cat $_{1}$ & cat $_{2}$ & cat $_{3}$ & $s s_{1}$ & $s s_{2}$ & $s s_{3}$ & $s s_{4}$ & $z n_{1}$ & $z n_{2}$ & $z n_{3}$ \\
\hline
\end{tabular}

Figura 101 Distribución genes en Genotipo CASO 1

\subsubsection{Configuración para la Discretización Zonal y Ponderación de elementos}

La distribución de pesos asignados se muestra a continuación.

- Ponderación de los elementos significativos:

\begin{tabular}{lllllll}
\hline Tipo Elemento & $\mathrm{OH}_{1}$ & $\mathbf{O H} L_{2}$ & $S S_{1}$ & $S S_{2}$ & $S S_{3}$ & $S S_{4}$ \\
\hline Peso asignado & 300 & 600 & 100 & 400 & 100 & 100 \\
\hline
\end{tabular}

Tabla 45 Pesos asignados a cada elemento

Los pesos de las catenarias han sido ponderados. La relación de 2:1 en precio, se ha estimado debido a que el material de la primera, conjuntamente con el tamaño de la sección y resistividad, permiten pensar que una es mucho mejor que la otra, y se ha dejado establecido en la relación ya comentada, al no poseer datos concretos de precios exactos. En cuanto a las subestaciones, al igual que en los otros casos, se ha determinado un peso distinto para seguir observando la esperada incidencia de la ponderación en la elección final de los elementos.

\begin{tabular}{lcccc}
\hline $\begin{array}{l}\mathbf{N}^{\text {O }} \\
\text { Zona }\end{array}$ & Tipo Zona & $\begin{array}{l}\text { Posición inicial } \\
(\mathbf{m})\end{array}$ & $\begin{array}{l}\text { Posición final } \\
(\mathbf{m})\end{array}$ & $\begin{array}{l}\text { Peso } \\
\text { asignado }\end{array}$ \\
\hline Zona1 & & 0 & 20000 & 135 \\
Zona2 & Instalación & 15000 & 20000 & 50 \\
Zona3 & & 30000 & 40000 & 90 \\
Zona4 & & 45000 & 60000 & 90 \\
Zona5 & Medioambiental & 9500 & 10500 & 100 \\
Zona6 & & 20000 & 30000 & 100 \\
\hline
\end{tabular}




\begin{tabular}{lcccc}
\hline $\begin{array}{l}N^{0} \\
\text { Zona }\end{array}$ & Tipo Zona & $\begin{array}{l}\text { Posición inicial } \\
(\mathbf{m})\end{array}$ & $\begin{array}{l}\text { Posición final } \\
(\mathbf{m})\end{array}$ & $\begin{array}{l}\text { Peso } \\
\text { asignado }\end{array}$ \\
\hline Zona7 & Conexión con la red & 40000 & 42500 & 3000 \\
Zona8 & general & 5000 & 17000 & 200 \\
Zona9 & & 25000 & 27000 & 200 \\
Zona10 & Mantenimiento & 1 & 1000 & 350 \\
Zona11 & & 10000 & 35500 & 650 \\
Zona12 & & 8000 & 8500 & 3350 \\
\hline
\end{tabular}

Tabla 46 Información completa acerca de la Discretización Zonal elegida

Respecto a la discretización zonal, la subdivisión de las zonas de instalación y el número de ellas se corresponde con el algoritmo diseñado. Por cada subestación equivale una zona de instalación y además todas deben tener la misma longitud, en este caso de $20 \mathrm{~km}$.

Al ser un proyecto ficticio de línea ferroviaria, es obvio que no existían datos fidedignos del impacto de Zonas respecto de la estimación global del proyecto. Al igual que en otros casos, se han asignado valores con el principal propósito de encontrar una pauta de comportamiento válido del algoritmo respecto de la ponderación. Asimismo se quiere comprobar que, en la medida de lo posible, el algoritmo evita la instalación en zonas con un alto coste ponderado, como son la zona 7 Medioambiental y la zona $12 \mathrm{de}$ Mantenimiento.

\subsubsection{Comentarios configuración inicial}

Esta herramienta puede ser usada tanto para casos de sobredimensionamiento inicial como para detectar problemas irresolubles del sistema. El planteamiento para este CASO ha sido sobredimensionar el sistema de energía, para ver cómo discurre el método y a qué conclusión se llega. Será interesante ver las opciones finales del Frente de Pareto cómo han excluido de la solución final alguna o varias subestaciones de tracción. Uno de los caminos para asegurar el efecto buscado, es modelar el plan de explotación con un tramo de menor longitud del que presenta la línea. El plan va a circular solo sobre 40km de la línea, es decir, comenzará en la STOP1 para finalizar en la STOP3.

Los resultados finales despejan dudas acerca del sobredimensionamiento diseñado inicialmente, como se va a detallar en el siguiente apartado.

\subsubsection{Proceso optimización del CASO 1. Configuración y resultados. Análisis.}

\subsubsection{Configuración parámetros del algoritmo genético.}

Para el CASO 1 se aplicó el algoritmo genético NSGA-II. Como primera aproximación al problema, se analizó el impacto de los parámetros determinantes en la búsqueda heurística del Frente óptimo. También se fueron evaluando el impacto, y la necesidad, de incrementar el número de individuos y poblaciones para poder examinar un rango mayor de posibilidades de escenarios de dimensionamiento eléctrico final. 
En la tabla 6 aparecen los valores asignados a los parámetros asociados a los operadores del algoritmo. Estos parámetros se fueron ajustando conforme se realizaban las pruebas y se comprobaba la efectividad del algoritmo.

\begin{tabular}{lllllll}
\hline Población & $\begin{array}{l}\text { No } \\
\text { Generaciones }\end{array}$ & $\begin{array}{l}\text { Probabilidad } \\
\text { de Cruce } \\
(0-1)\end{array}$ & $\begin{array}{l}\text { Probabilidad } \\
\text { mutación } \\
(0-1)\end{array}$ & $\begin{array}{l}\text { Valor índice } \\
\text { distribución } \\
\text { de } \\
\text { mutación } \\
(0-100)\end{array}$ & $\begin{array}{l}\text { Valor índice } \\
\text { distribución } \\
\text { cruce } \\
(0-100)\end{array}$ & $\begin{array}{l}\text { Factor } \\
\text { eliminación } \\
\text { subestación }\end{array}$ \\
\hline $\begin{array}{l}20 \\
\text { individuos } \\
40\end{array}$ & 20 & 0.7 & 0.25 & 350 & 25 & -0.15 \\
$\begin{array}{l}\text { individuos } \\
80\end{array}$ & 20 & 0.8 & 0.25 & 400 & 30 & -0.2 \\
$\begin{array}{l}80 \\
\text { individuos } \\
80\end{array}$ & 20 & 0.85 & 0.2 & 350 & 25 & -0.15 \\
$\begin{array}{l}\text { individuos } \\
80\end{array}$ & 20 & 0.75 & 0.25 & 400 & 30 & -0.2 \\
\hline individuos & 35 & 0.8 & 0.25 & 400 & 35 & -0.3 \\
\hline
\end{tabular}

Tabla 47 Valor parámetros finales NSGA-II CASO 1

La estimación de la probabilidad de cruce y mutación se pensó para incentivar la diversidad genética, ya que son valores muy altos y por tanto, permiten en gran medida la aplicación de ambos operadores. Los valores de distribución son muy cercanos al estándar y el de factor de eliminación no es un valor muy alto, ronda entre el 15 y el $20 \%$ del incremento. 
6.3.8.2 Resultados y análisis. Conclusiones.
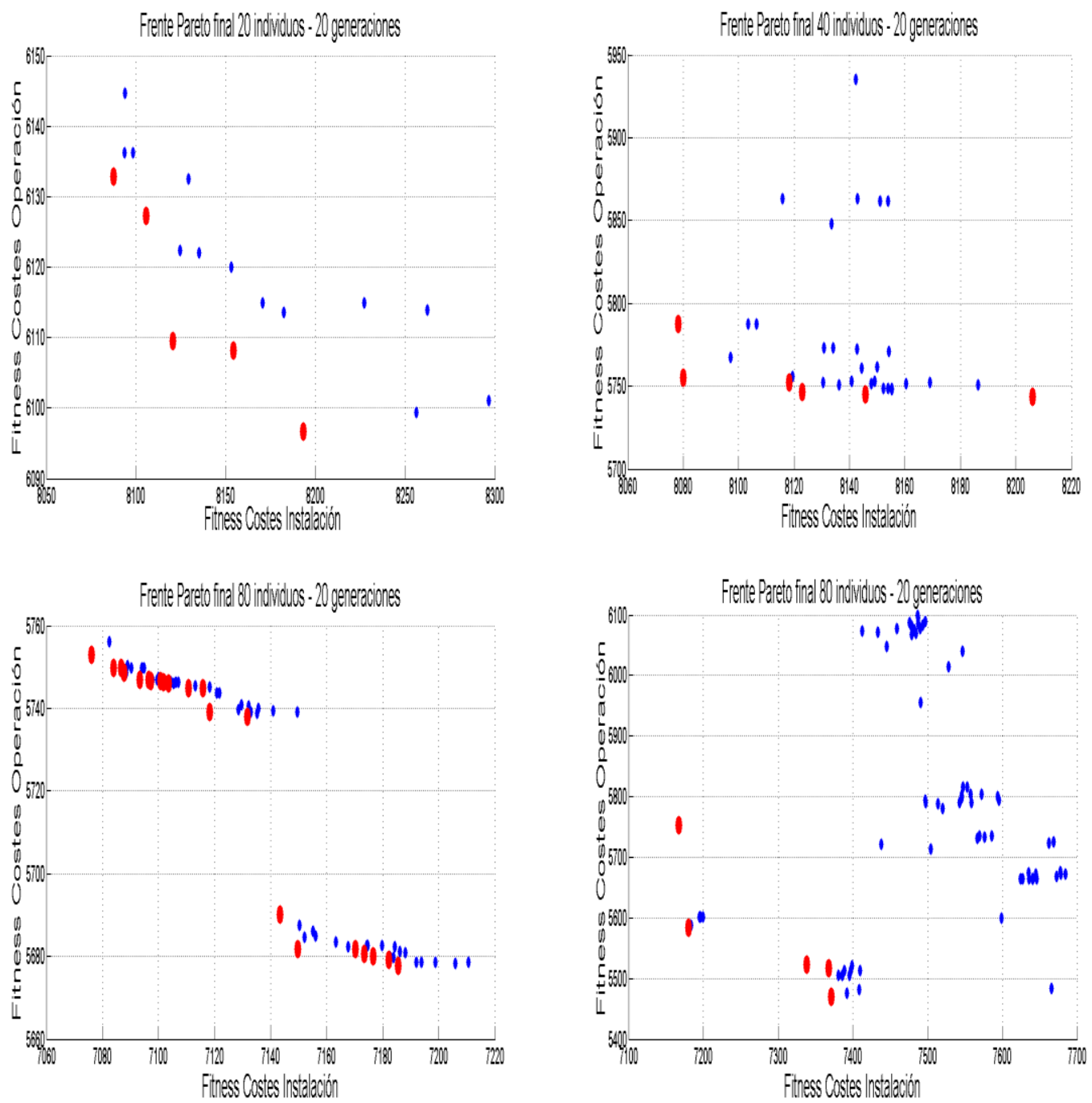

Figura 102 Frentes de Pareto finales para tests previos. a) test 20 individuos 0.7 pcross, 0.25 pmut, -0.15 elim_ss; b) test 40 individuos 0.8 pcross, 0.25 pmut, $-\mathbf{- 0 . 2}$ elim_ss; c) 80 individuos 0.85 pcross, 0.2 pmut, -0.15 elim_ss; d) 80 individuos 0.75 pcross, 0.25 pmut, -0.2 elim_ss

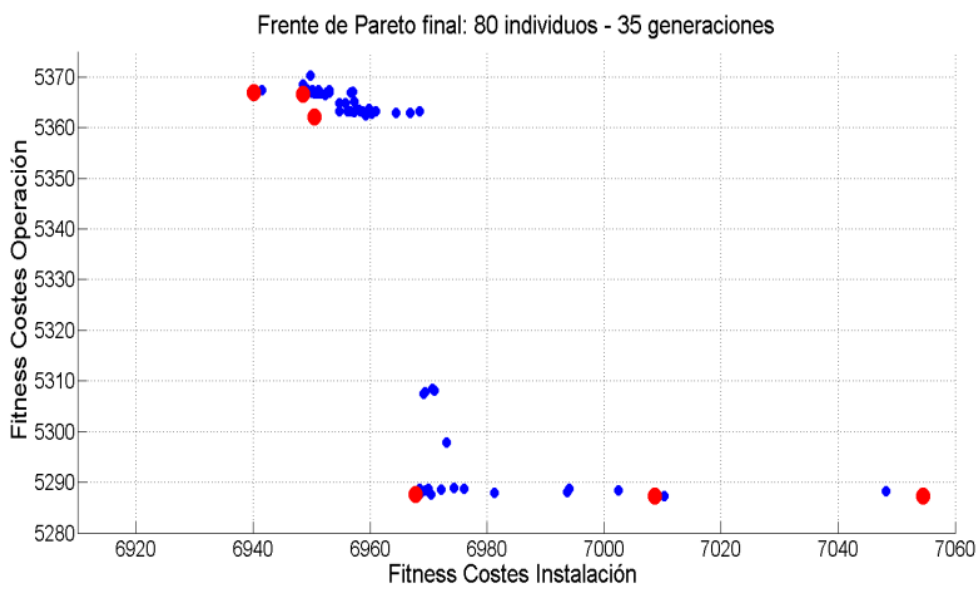

Figura 103 Frentes última generación prueba final 
Previo paso a analizar los resultados del algoritmo genético, es importante describir la máquina sobre la que se han realizado las pruebas y los tiempos de cálculo recogidos. Para no desvirtuar el trabajo de investigación con diferentes máquinas, se siguió realizando las simulaciones y optimizaciones con la misma máquina que se describió en el Capítulo 5. A continuación, en la tabla siguiente se muestran los valores de cálculo más relevantes:

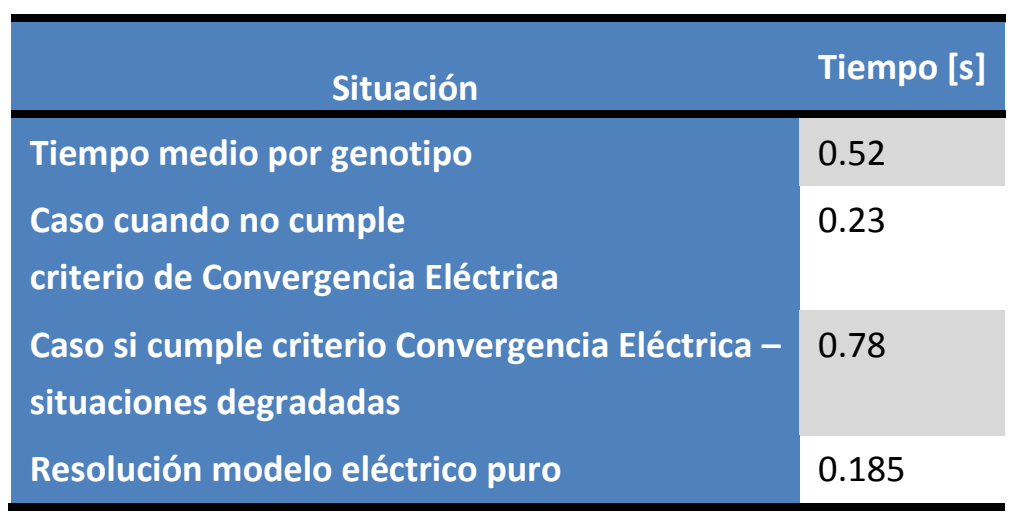

Tabla 48 Tiempos de cálculo para situaciones corriente alterna

En las figuras anteriores se ven reflejados los resultados finales del algoritmo de las pruebas realizadas. En azul se muestran los genotipos dominados y los rojos son los que forman el conjunto de no-dominados, y por tanto el Frente de Pareto, o solución final.

Debido al sobredimensionamiento que se presuponía ya por el diseño inicial, se ha configurado con un valor de eliminación de subestación muy alto, proporcionalmente hablando. Esto debe incidir en descartar alguna de las subestaciones que componen la configuración inicial. Los otros parámetros, también se han valorado bastante elevados, con la intención de buscar un rango mayor en el espacio de soluciones de la configuración.

En términos de examinar la diversidad de las soluciones aportadas, se examina que en las configuraciones a) y b), cuando menor es el número de la población, las soluciones finales son más homogéneas, en cuanto a ocupar todas un área más o menos reconocible. Conforme se ha incrementado el número de individuos, el algoritmo ha dividido las soluciones en dos frentes diferenciados completamente, como se observa muy claramente en la figura c) y en la Figura 103 Frentes última generación prueba final. Si se realiza un examen más profundo, se verá que los valores objetivo finales, a pesar de que están separados espacialmente, cuantitativamente no es tan grande la diferencia en términos relativos. Aparte de los valores de distribución de los operadores, es muy probable que al ser tan pequeño el área diferencial que se ha asignado como valor inicial, que aunque se prolongaran las generaciones, siempre surgiría esa disparidad espacial que se observa en los gráficos de la Figura 5. Por otra parte este espaciamiento permite analizar diversas soluciones distintas, y por tanto se gana en cuanto a la gran diversidad de las posibles soluciones a tener en cuenta para el escenario final. 
En cuanto a los valores resultantes de los fenotipos, se constata una mejora substancial conforme se va incrementando la potencia de cálculo del algoritmo. Mientras que para 20 individuos se encuentra un baremos de solución entre [8100,6050] para mejores valores de cada función objetivo, en la solución final ese baremo pasa a mejorar casi un $20 \%$, quedándose en [6940, 5280].

Es importante señalar que en todas las soluciones aportadas para todos los casos, el Frente de Pareto obtiene soluciones que cumplen las restricciones. Es de importancia, ya que como corolario se puede afirmar que con un procesamiento rápido de la metodología, que implica un bajo nivel relativo de individuos y de generaciones, sumado a un alto nivel del búsqueda de diversidad generacional, en este caso, se obtendría un conjunto de soluciones de dimensionamiento. En caso de querer obtener una mejor opción, es necesario aumentar la población proporcionalmente al número de genes. De esta manera se comprueban más opciones y el algoritmo es más concluyente.

La elección en este caso dependerá de la necesidad inmediata de obtener solución. Como se verá más adelante, para un problema de este tamaño, se debería siempre explorar el proceso, hasta un límite cercano al marcado por los parámetros finales aplicados en este test. 


\subsubsection{Diseño del sistema eléctrico utilizando genotipo con mejor valor función objetivo Costes de Operación}

La configuración propuesta por el genotipo es la siguiente:

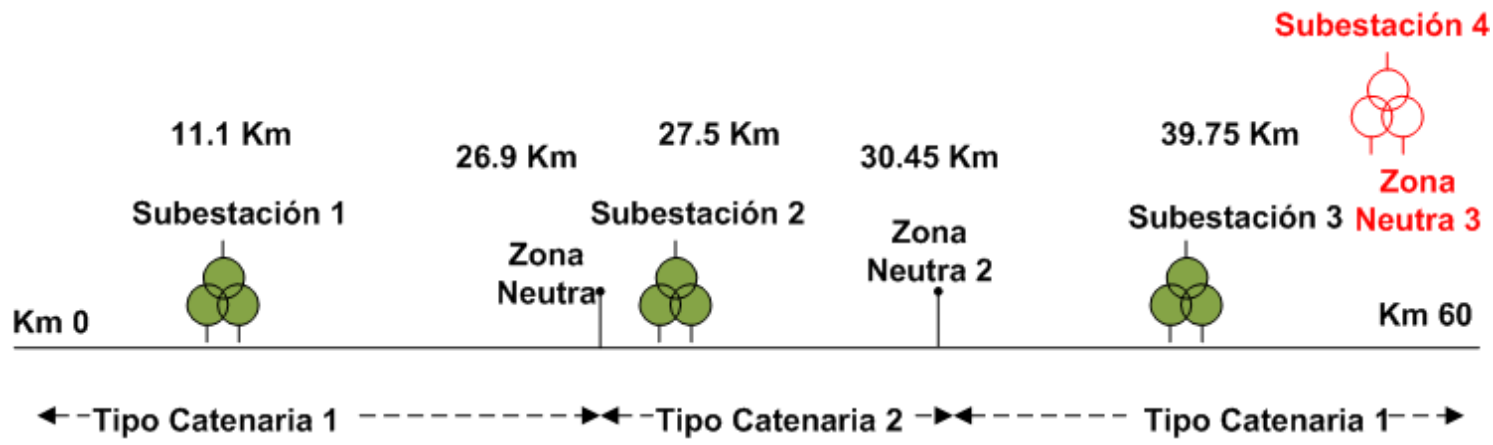

Figura 104 Configuración para mejor valor para costes de instalación CASO 1

Los análisis previos al proceso de optimización, en los cuales se intuía un sobredimensionamiento, provocado, se han confirmado con el diseño proporcionado por el genotipo elegido para este apartado. La subestación 4 quedaría fuera del dimensionamiento eléctrico, y por tanto, la zona neutra asociada no sería necesaria instalarla.

Los tipos de catenaria escogidos ha seguido un criterio de reforzar la parte central, que es donde se concentra la mayor parte del tráfico, mejorando así las pérdidas en la línea. Esto a pesar del sobrecoste que implica la preferencia de una sobre la otra, el algoritmo encuentra mejor esa opción final.

Tensiones mínimas: Catenaria

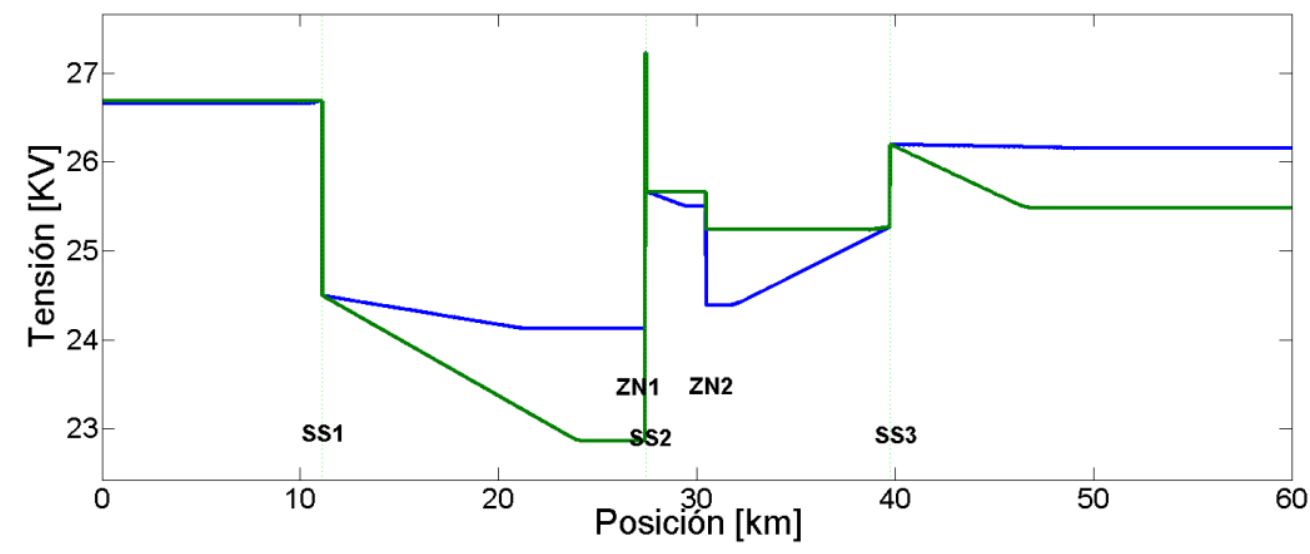

Figura 105 Tensiones mínimas para escenario con mejor valor para costes de operación 


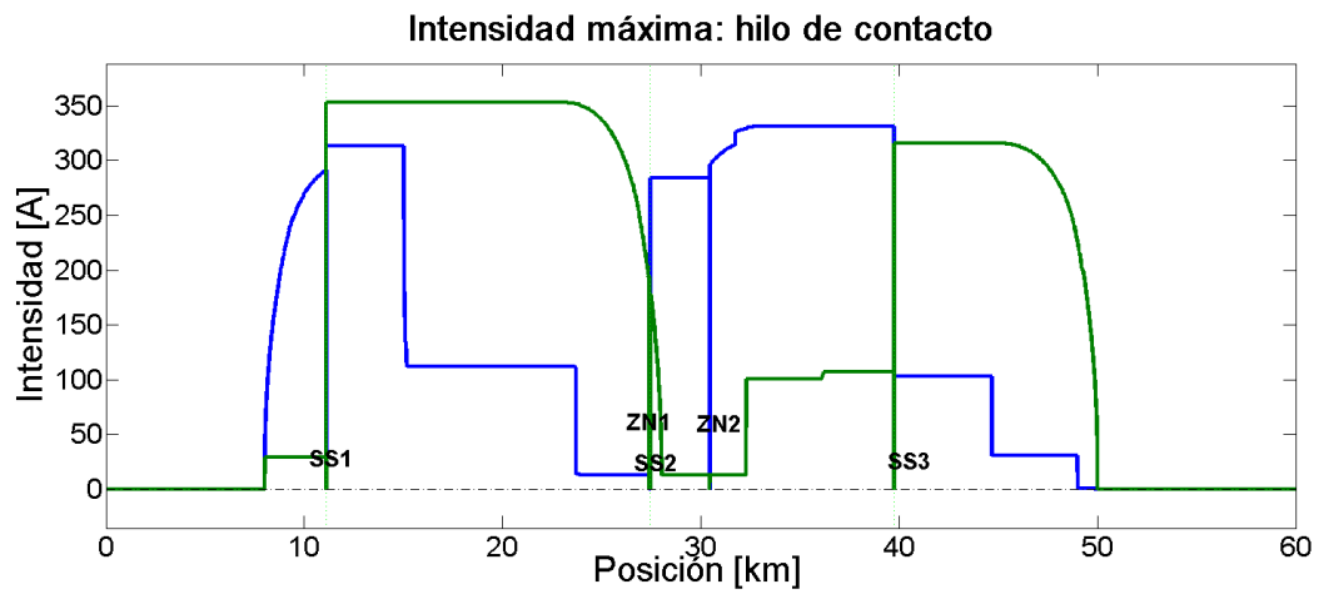

Figura 106 Intensidades máximas para escenario con mejor valor para costes de operación

En las gráficas anteriores se puede observar que donde hay más exigencia para el sistema eléctrico es en la zona eléctrica entre las subestaciones 1 y 2 . A continuación se van a mostrar los resultados de las tensiones en catenaria para las situaciones degradadas y un ejemplo de intensidad máxima, también para situación degradada.
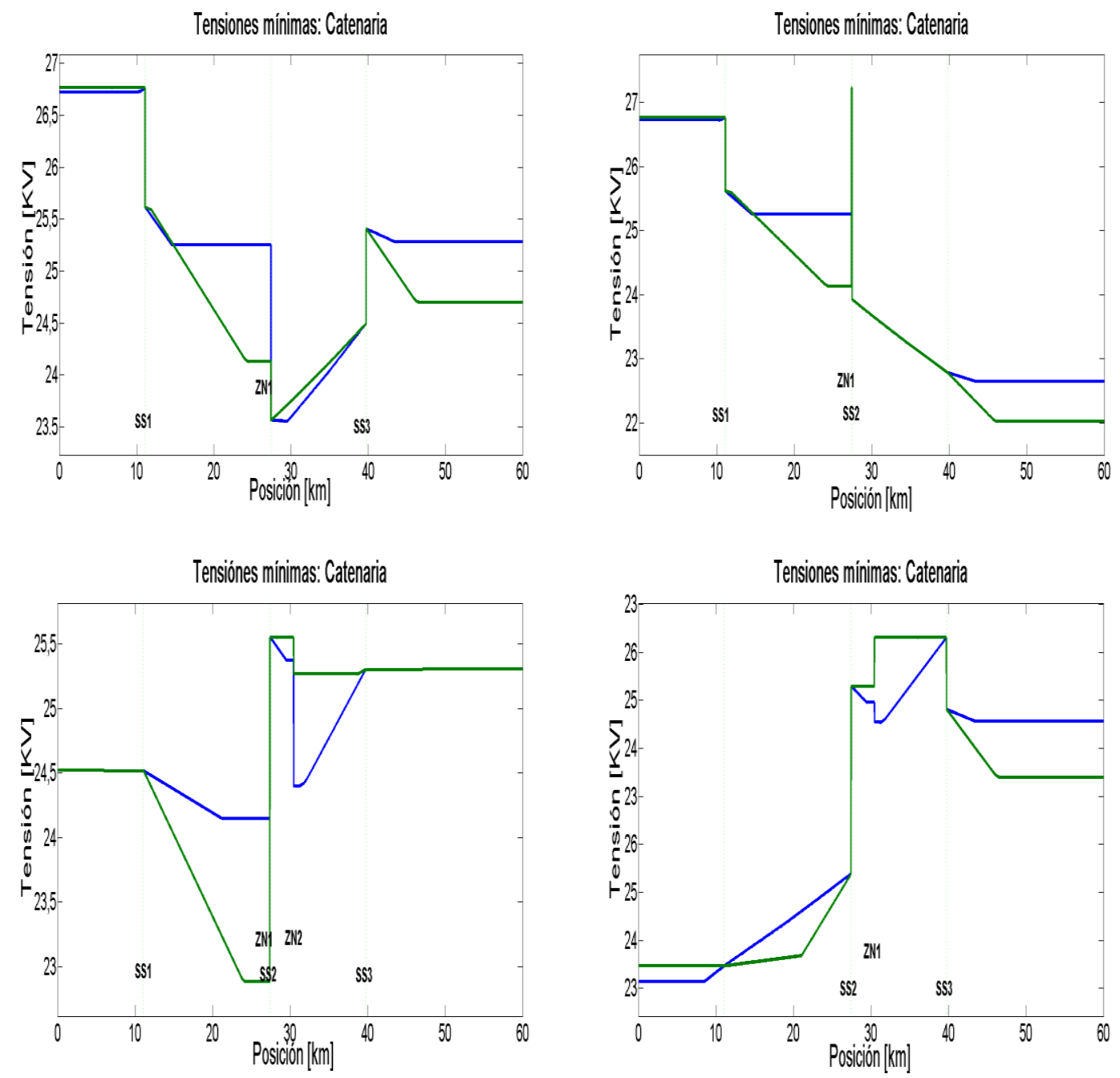

Figura 107 Tensiones mínimas en catenaria para situaciones degradadas de mejor caso para costes operación. a) $\sin \mathrm{SS} 2$;b) $\sin \mathrm{SS3}$;c) degradada simple; d) $\sin \mathrm{SS1}$ 
Intensidad máxima: hilo de contacto

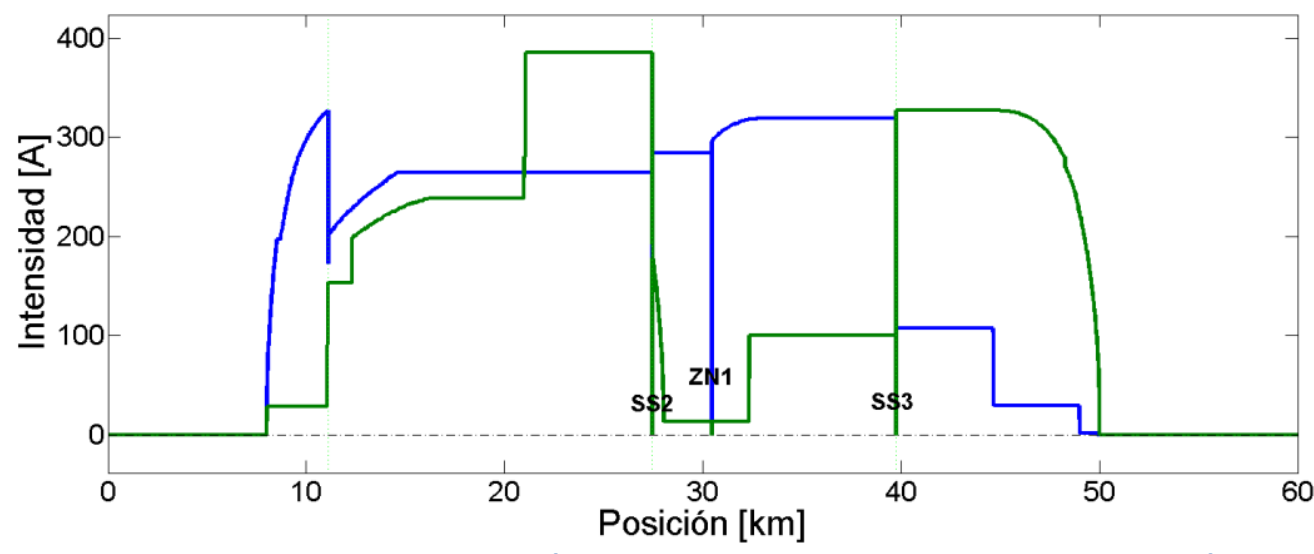

Figura 108 Intensidad máxima para caso degradado completo de subestación 1

Analizando el conjunto de gráficas de tensiones mínimas se observa que la caída más grande se produce cuando la subestación 3 se queda fuera de servicio. La tensión se queda bordeando los $22 \mathrm{kV}$. El resto de gráficas muestran valores por encima, por lo que la solución aportada por el algoritmo, eliminando una subestación, se podría dar por satisfactoria, teniendo en cuenta el análisis inicial que se ha considerado. Las intensidades máximas, debido a las características de la línea de electrificación, han sido recogidas y son relativamente bajas, no llegado a los $400 \mathrm{~A}$.

\subsubsection{Diseño para mejor valor función objetivo Costes de Instalación}

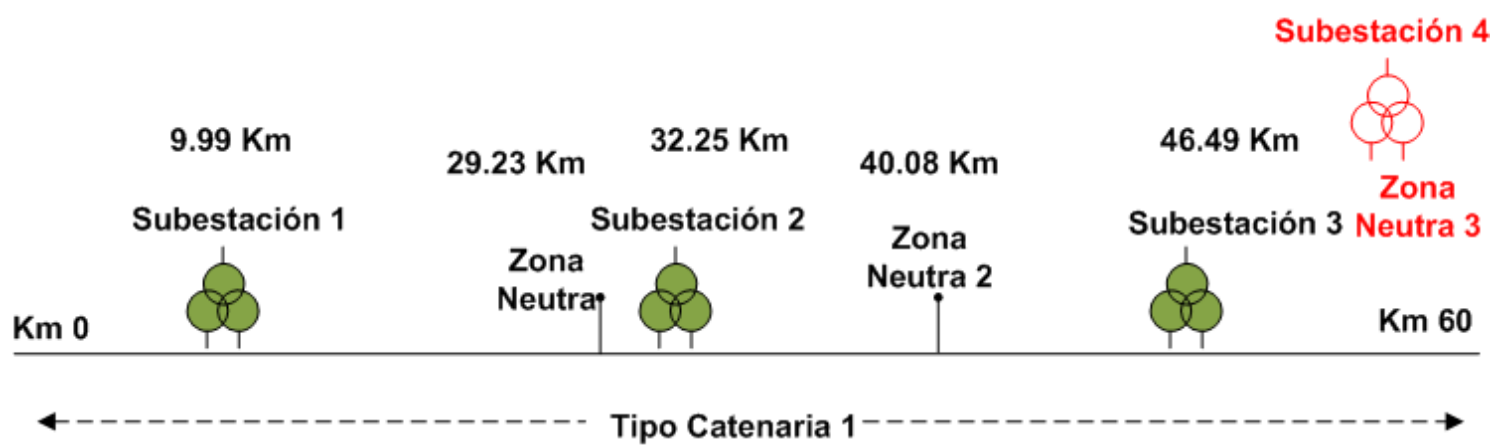

Figura 109 Configuración para mejor valor para costes de instalación CASO 1

Se muestra la solución mejor para valor de función objetivo asociado a costes de Instalación. Los resultados finales son muy parejos a la solución anterior, en cuanto a diseño final del dimensionamiento y posición. Quizá lo más destacado es la opción de instalar la catenaria solo con el Tipo 1, que es menos costoso, y por tanto decrementa el valor total focalizado en esta función objetivo. 

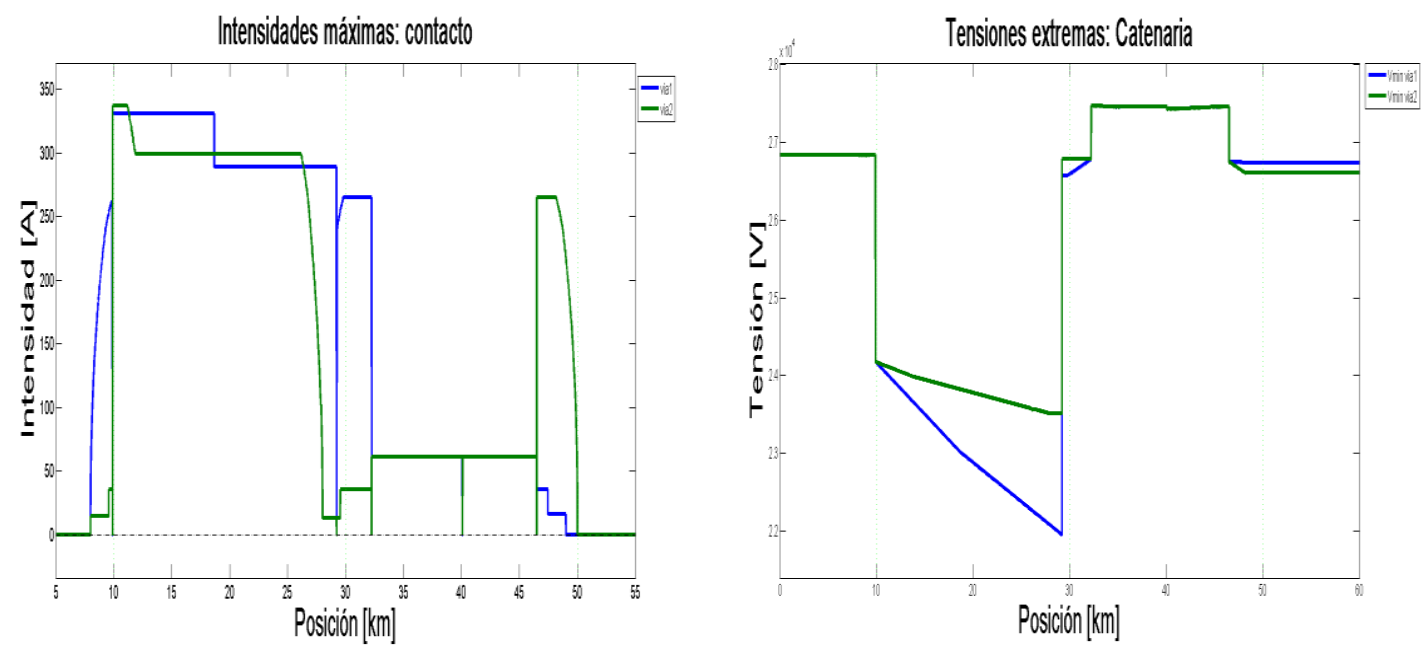

Figura 110 Resultados eléctricos situación normal. a) intensidades máximas; b) tensiones mínimas

La simulación del escenario con el dimensionamiento optimizado, ha resultado como más relevante en las gráficas 13 a y 13 b.
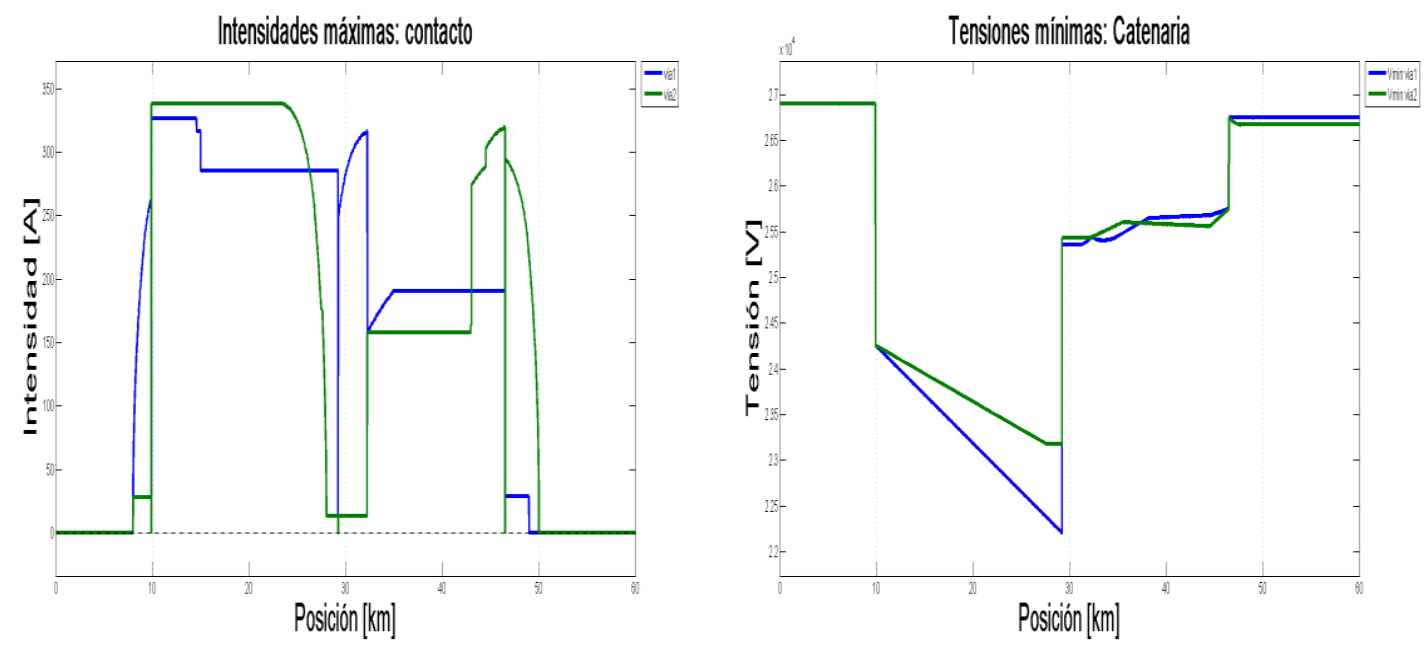

Figura 111 Resultados eléctricos situación degradada completa subestación 2. a) intensidades máximas; b) tensiones mínimas

Los resultados eléctricos no difieren mucho de los cosechados en el estudio para el mejor valor con coste de instalación. Se han seleccionado las gráficas de intensidades y tensiones del escenario con la subestación 2 fuera de servicio. La caída de la subestación provoca a su vez una caída de tensión en el ramal eléctrico afectado, pero no implica una caída importante, de cara a peligrar el límite normativo. En cuanto a las intensidades, no se prevén problemas ya que de nuevo, son intensidades relativamente bajas.

\subsubsection{Comparación y conclusiones}

Se repite el comportamiento y análisis de anteriores casos referidos a los tiempos de obtención de resultados por medio de esta metodología. Mientras que para una sola simulación, se puede consumir alrededor de los 50 minutos, para el proceso global de 
optimización, dependiendo obviamente de la población y generaciones a examinar por el algoritmo NSGA-II, se obtendría en unas 3-4 horas.

El sistema propuesto en este CASO estaba diseñado para provocar un sobredimensionamiento eléctrico en la línea. Esto se debía manifestar en una disminución de los elementos involucrados. En el Frente de Pareto final con mejor ránking, las soluciones optaban siempre por eliminar una subestación. En ambos casos mostrados, la subestación 4.

La comparación de los resultados elegidos deviene en una similitud bastante alta. La subestación 4 es la que el algoritmo decide eliminar en ambos casos. A nivel de estudio de parámetros eléctricos, ambas soluciones tienen características también similares en cuanto a nivel de caídas de tensión y de intensidades máximas, entre otros. La variación de las configuraciones no es significativa, salvo para aumentar o disminuir en pequeña medida los valores de los fenotipos de la funciones objetivo examinadas.

A pesar de no tener muchos genes el genotipo, se ha demostrado la mejora del resultado final con una variedad poblacional mayor. Este aumento poblacional provoca que el método prolongue más tiempo la búsqueda del óptimo. En casos como el visto en el capítulo 5 y el siguiente, caso 2 de corriente alterna, se deberá tener en cuenta el tamaño del problema y el tiempo para obtener una solución, confrontándolo con la mejora que se desea, ya que es probable que con una dimensión pequeña del espacio de búsqueda, se obtenga una solución satisfactoria. 


\subsection{CASO 2}

\subsubsection{Infraestructura de la línea}

La línea pertenece a un proyecto real, a partir del cual se han extraído todos los datos necesarios para aplicar la Metodología. La línea tiene una longitud aproximada de $210 \mathrm{~km}$ y al ser un sistema pensado para alta velocidad, el plan diseñado no contempla paradas durante el recorrido. Limitaciones de velocidad incluidas en la configuración. El tipo de sistema ferroviario, alta velocidad, implica que uno de los objetivos es mantener la velocidad durante el recorrido. Para facilitar esta tarea al material rodante, el alzado no suele tener grandes pendientes, por lo que las fuerzas resistentes serán menor y el esfuerzo a realizar también será menor que en un trazado con fuertes pendientes positivas. La limitación de velocidad en la línea es de $300 \mathrm{~km}$. $/ \mathrm{h}$

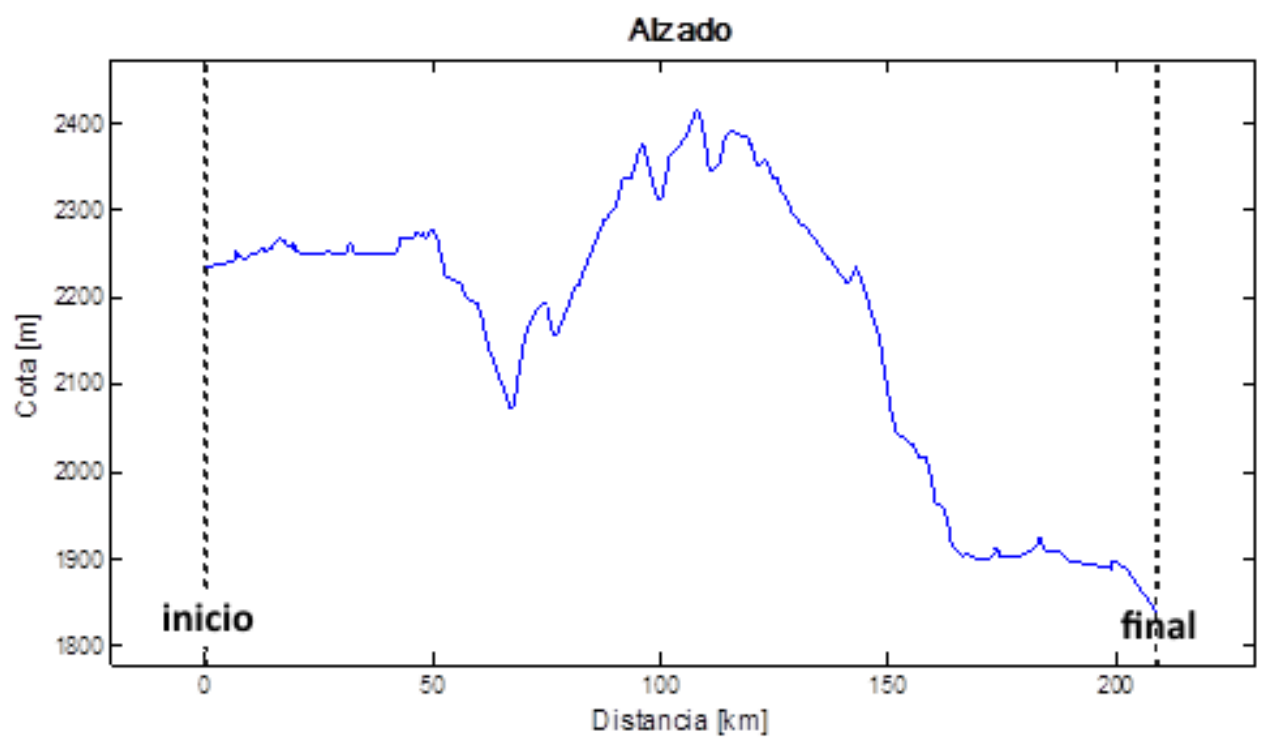

Figura 112 Perfil del alzado para la línea ferroviaria usada en CASO 2

\subsubsection{Material rodante}

Configuración de la distribución de los coches del tren:

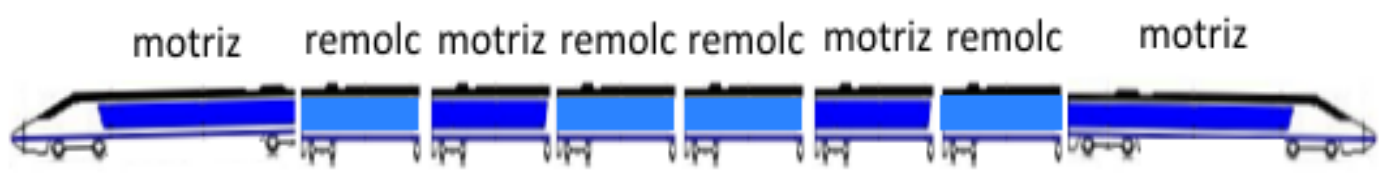

Figura 113 Composición del tren para CASO 2

M-R-M-R-R-M-R-M $\rightarrow$ Dos coches motrices cabeceros, dos intermedios y los 4 restantes no aportan tracción. 


\begin{tabular}{|c|c|}
\hline Propiedades & Valores \\
\hline Masa & $495 \mathrm{Tm}$ \\
\hline Masas Giratorias & $50 \mathrm{Tm}$ \\
\hline Pasajeros & 500 \\
\hline Coeficientes resistencia avance & $\begin{array}{c}\mathrm{A}[\boldsymbol{N}]=\mathbf{3 5 7 0} \\
\mathrm{B}[\boldsymbol{N} * \boldsymbol{s} / \boldsymbol{m}]=\mathbf{1 2 0 . 3 4 4} \\
\mathrm{C}\left[\boldsymbol{N} * \boldsymbol{s}^{2} / \boldsymbol{m}^{2}\right]=7.7295\end{array}$ \\
\hline Resistencia en curva (Kc) & 500 \\
\hline Deceleración emergencia & $0.8^{\mathrm{m}} / \mathrm{s}^{2}$ \\
\hline Deceleración servicio & $0.56^{m} / s^{2}$ \\
\hline Aceleración tracción & $0.55^{\mathrm{m}} / \mathrm{s}^{2}$ \\
\hline Aceleración confort (jerk) & $0.75 \mathrm{~m} / \mathrm{s}^{2}$ \\
\hline Distancia deslizamiento & $8 \mathrm{~m}$ \\
\hline Velocidad máxima & $300 \mathrm{~km} / \mathrm{h}$ \\
\hline
\end{tabular}

Tabla 49 Propiedades material rodante CASO 2

Las características habituales que se han conformado para crear el modelo del tren y poder llevar a cabo las simulaciones y demandas de potencia eléctrica del material rodante en circulación.

\subsubsection{Señalización}

ERTMS-2 idéntico sistema integrado que para el CASO 1, que por otra parte es muy usado en sistemas ferroviarios de este tipo.

\subsubsection{Sistema eléctrico inicial}

Al tratarse de una línea de alta velocidad, se ha implantado uno de los sistemas eléctricos más comunes para este tipo de líneas ferroviarias, $50 \mathrm{~Hz}$ y tensión $25 \mathrm{kV}$ (se alimenta desde los $27,5 \mathrm{kV}$ para asegurar en catenaria los $25 \mathrm{kV}$ ). Esta línea ferroviaria tiene una particularidad y es que tiene una zona inicial en que funciona hasta el momento en sistema monotensión, 1x25, y el resto en 2x25.

Se ha trabajado con dos tipos de catenaria distintos. En la tabla siguiente se pueden examinar los diferentes cables usados y características más relevantes de cada uno como material, sección o resistividad.

\begin{tabular}{lllrl}
\hline \multicolumn{1}{c}{ Conductor } & Catenaria & Material & Sección & Resistividad \\
\hline Sustentador & OHL 1 & $\mathrm{Cu}$ & $95 \mathrm{~mm}^{2}$ & $0,02778 \mu \Omega \cdot \mathrm{m}$ \\
Contacto & OHL 1 & $\mathrm{Cu} \mathrm{Mg}$ & $150 \mathrm{~mm}^{2}$ & $0,02778 \mu \Omega \cdot \mathrm{m}$ \\
Sustentador & OHL 2 & $\mathrm{Cu}$ & $120 \mathrm{~mm}^{2}$ & $0,02778 \mu \Omega \cdot \mathrm{m}$ \\
Contacto & OHL 2 & $\mathrm{Cu} \mathrm{Mg}$ & $200 \mathrm{~mm}^{2}$ & $0,02778 \mu \Omega \cdot \mathrm{m}$ \\
\hline
\end{tabular}




\begin{tabular}{ccccc}
\hline \multicolumn{1}{c}{ Conductor } & Catenaria & Material & Sección & Resistividad \\
\hline Carril estándar & OHL 1/OHL 2 & UIC 60 & $7693 \mathrm{~mm}^{2}$ & $0.207 \mu \Omega \cdot \mathrm{m}$ \\
Feeder Negativo & OHL 1/OHL 2 & LA 280 & $281.1 \mathrm{~mm}^{2}$ & $0,02887 \mu \Omega \cdot \mathrm{m}$ \\
\hline
\end{tabular}

Tabla 50 Características básicas catenarias utilizadas

La intención era comprobar cómo aplicaba la metodología el conjunto de catenarias, teniendo una sensiblemente mejor que la otra, aunque como se verá en la distribución de pesos, un poco más cara también.

La configuración inicial constaba de instalación de 5 subestaciones de tracción y de 16 autotransformadores, tanto intermedios como final de zona eléctrica. Cada subestación proporciona una potencia de 40 MVA y los autotransformadores intermedios de 2x10 MVA y los finales de zona 4x10 MVA.

La configuración inicial es la siguiente:

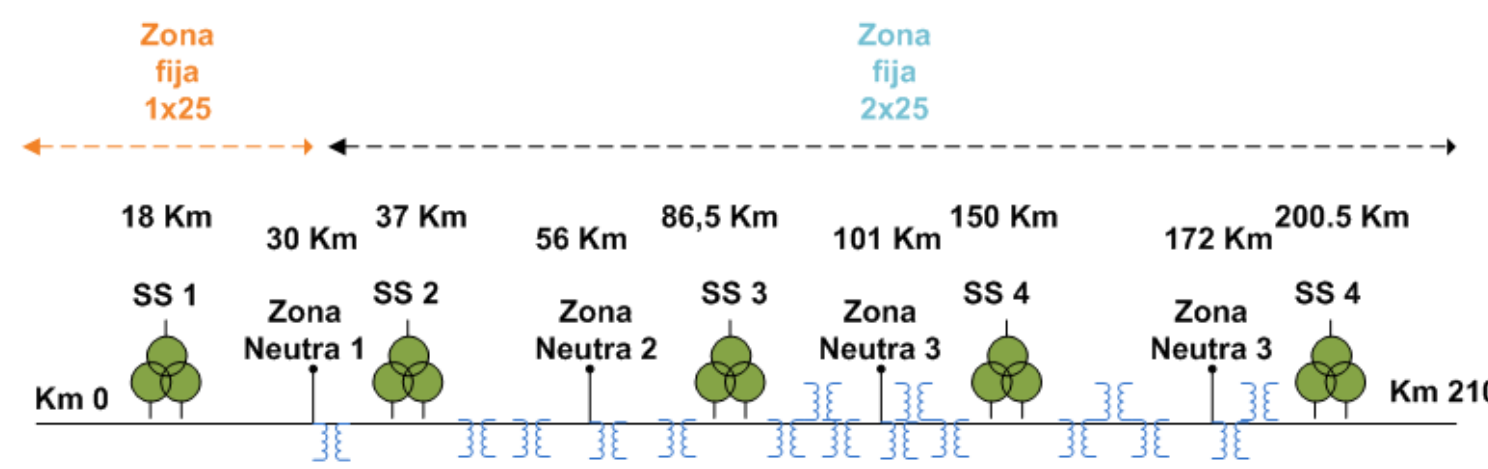

Autotransformador

Catenaria Tipo 1

Figura 114 Diagrama explicativo dimensionamiento eléctrico inicial

\subsubsection{Plan de operación de la línea}

- Los trenes deben cumplir un intervalo de 20 minutos tanto para la situación normal como para situación degradada de funcionamiento del sistema eléctrico.

- El plan de explotación no contempla paradas intermedias, por lo que el tráfico es directo entre la estación inicial y final. Los cambios de cabina y reanudación de la nueva circulación tiene un tiempo estimado de 639 segundos.

- La flota que se pone en circulación es de 7 trenes. 


\subsubsection{Configuración algoritmo genético.}

En este caso sí que existen ciertas zonas que ya sea por condiciones medioambientales o por zonas de difícil acceso para la conexión a la red general, se han explicitado como muy complicadas de situar un elemento eléctrico del dimensionamiento. Además para dar más riqueza a los resultados obtenidos por los algoritmos genéticos, se han aumentado las posibilidades de discretización y por tanto, se tratará de comprobar la diferencia respecto a la uniformidad encontrada en los resultados del CASO2 de corriente continua.

Para este ejemplo se han utilizado los tres algoritmos genéticos.

\subsubsection{Genotipo}

Al ser un sistema dual en alterna, y como ya se explicó en el Capítulo 4, se debe pensar en un genotipo completo:

- 4 tipos de zonas de catenaria distintas

- 5 subestaciones de tracción

- 4 zonas neutras posibles

- 11 zonas posibles de autotransformadores

- Como máximo se ha establecido que se podrán instalar 3 autotransformadores en cada zona.

\begin{tabular}{|l|l|l|l|c|c|c|c|c|c|c|c|c|}
\hline cat $_{1}$ & cat $_{2}$ & cat $_{3}$ & cat $_{4}$ & $s s_{1}$ & $s s_{2}$ & $s s_{3}$ & $s S_{4}$ & $s s_{5}$ & $z n_{1}$ & $z n_{2}$ & $z n_{3}$ & $z n_{4}$ \\
\hline $\operatorname{trf}_{1}$ & $\operatorname{trf}_{2}$ & $\operatorname{trf} f_{3}$ & $\operatorname{trf}_{4}$ & $\operatorname{trf} f_{5}$ & $\operatorname{trf}$ & $\operatorname{trf}_{7}$ & $\operatorname{trf} f_{8}$ & $\operatorname{trf} f_{9}$ & $\operatorname{trf} f_{10}$ & $\operatorname{trf} f_{11}$ & & \\
\hline
\end{tabular}

Figura 115 Distribución genes en Genotipo CASO 2

\subsubsection{Configuración para la Discretización Zonal y Ponderación de elementos}

La distribución de pesos asignados se muestra a continuación.

- Ponderación de los elementos significativos:

\begin{tabular}{llllllllll}
\hline Tipo Elemento & $O H L_{1}$ & $O H L_{2}$ & $S S_{1}$ & $S S_{2}$ & $S S_{3}$ & $S S_{4}$ & $S S_{5}$ & $A T_{i}$ & $A T_{f}$ \\
\hline Peso asignado & 300 & 500 & 500 & 500 & 500 & 500 & 500 & 500 & 700 \\
\hline
\end{tabular}

Tabla 51 Pesos asignados a cada elemento

El peso de la catenaria se ha pensado que instalar una sección mayor siempre es más costoso, por lo que si se puede evitar, sería la opción mejor. La ponderación es mucho más elevada por esta razón. En cuanto a las subestaciones, se quiere comprobar cuál es el comportamiento teniendo todas un peso similar. Por último, es evidente que los autotransformadores menos potentes que son los intermedios debe tener un coste menor que los que soportan la posible caída de tensión en los intercambios de zonas eléctricas, divididas por zonas neutras. 


\begin{tabular}{lcccc}
\hline $\begin{array}{l}\text { No } \\
\text { Zona }\end{array}$ & Tipo Zona & $\begin{array}{l}\text { Posición inicial } \\
(\mathbf{m})\end{array}$ & $\begin{array}{l}\text { Posición final } \\
(\mathbf{m})\end{array}$ & $\begin{array}{l}\text { Peso } \\
\text { asignado }\end{array}$ \\
\hline Zona1 & Instalación & 0 & 30000 & 135 \\
Zona2 & & 30000 & 75000 & 135 \\
Zona3 & & 75000 & 120000 & 135 \\
Zona4 & & 120000 & 165000 & 135 \\
Zona5 & & 165000 & 206000 & 135 \\
Zona6 & Medioambiental & 0 & 3250 & 10000 \\
Zona7 & & 93500 & 99750 & 10000 \\
Zona8 & & 162000 & 162500 & 10000 \\
Zona9 & & 129000 & 135000 & 10000 \\
Zona10 & Conexión con la red & 10000 & 155000 & 1200 \\
Zona11 & general & 45000 & 50000 & 1300 \\
Zona12 & & 87000 & 89000 & 5000 \\
\hline Zona13 & & 112000 & 118000 & 3000 \\
Zona10 & Mantenimiento & 145000 & 150000 & 2500 \\
Zona11 & & 190000 & 193000 & 1200 \\
Zona12 & & 195000 & 198000 & 1200 \\
\hline
\end{tabular}

Tabla 52 Información completa acerca de la Discretización Zonal elegida

Respecto a la discretización zonal, la subdivisión de las zonas de instalación y el número de ellas se corresponde con el algoritmo diseñado. La primera zona debe corresponder con la zona eléctrica $1 \times 25$, que finaliza en el punto kilométrico 30. Las siguientes tienen una longitud bastante parecida para poder situar una subestación en cada posición posible.

Las zonas medioambientales todas son reales, y todas tienen un peso muy elevado para evitar en la mayor medida posible la inclusión de algún elemento dentro de las mismas. También existía una zona de conexión con la red general con un alto valor restrictivo, como se puede observar en la tabla. Los demás valores y áreas de zonas, son todas ficticias y todas con valores menores de las restrictivas por las condiciones iniciales del proyecto. En las soluciones finales analizadas, se comprobará si en algún caso se ha tenido que violar esta alta restricción inicial.

\subsubsection{Resultados eléctricos configuración inicial}

Siguiendo el esquema de trabajo del algoritmo de funcionamiento de la Metodología, se estudia el comportamiento del escenario inicial, que en este caso se corresponde con el dimensionamiento inicial propuesto en el proyecto. Las comprobaciones de posibles fallos de funcionamiento llevarían a que la optimización posiblemente no sería capaz de ahorrar en elementos eléctricos y tan solo podría manejar cambios de posicionamiento de subestaciones o autotransformadores para chequear si subsanaría los errores iniciales. Por el contrario, si los resultados son correctos, se podría analizar un posible sobredimensionamiento inicial, que el optimizador debería ser capaz de mejorarlo, tanto en costes de instalación como de mantenimiento.

Para esta comprobación incluimos las gráficas de tensiones mínimas en catenariapantógrafo y las tensiones de retorno obtenidas. 


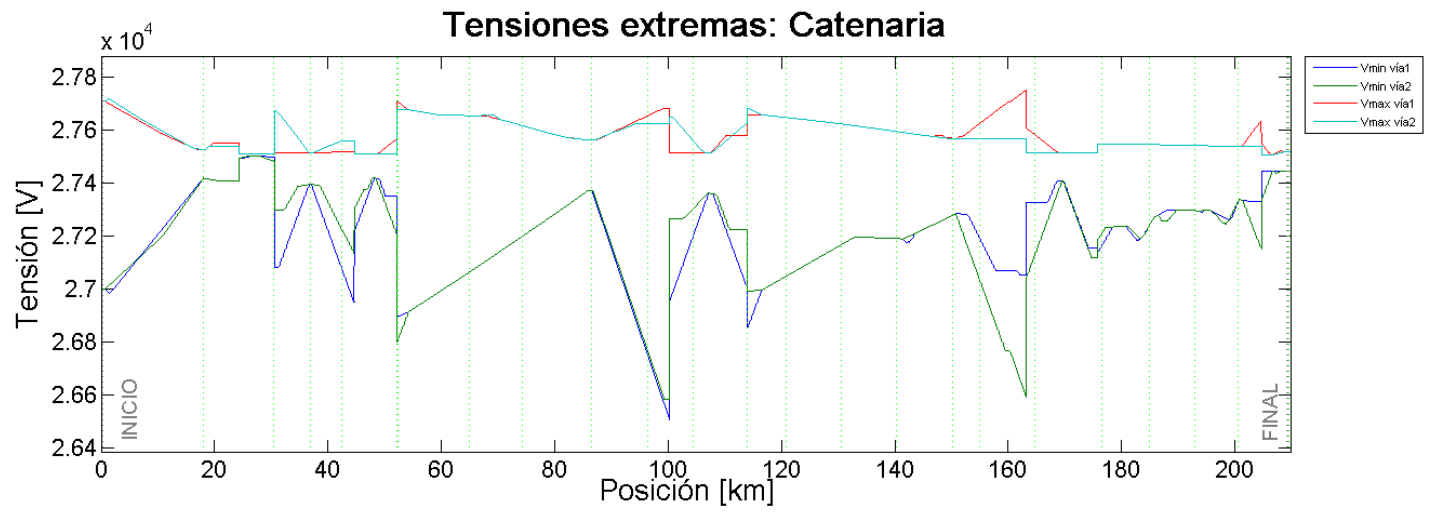

Figura 116 Tensiones extremas dimensionamiento inicial CASO 2

Tensiones extremas: Retorno

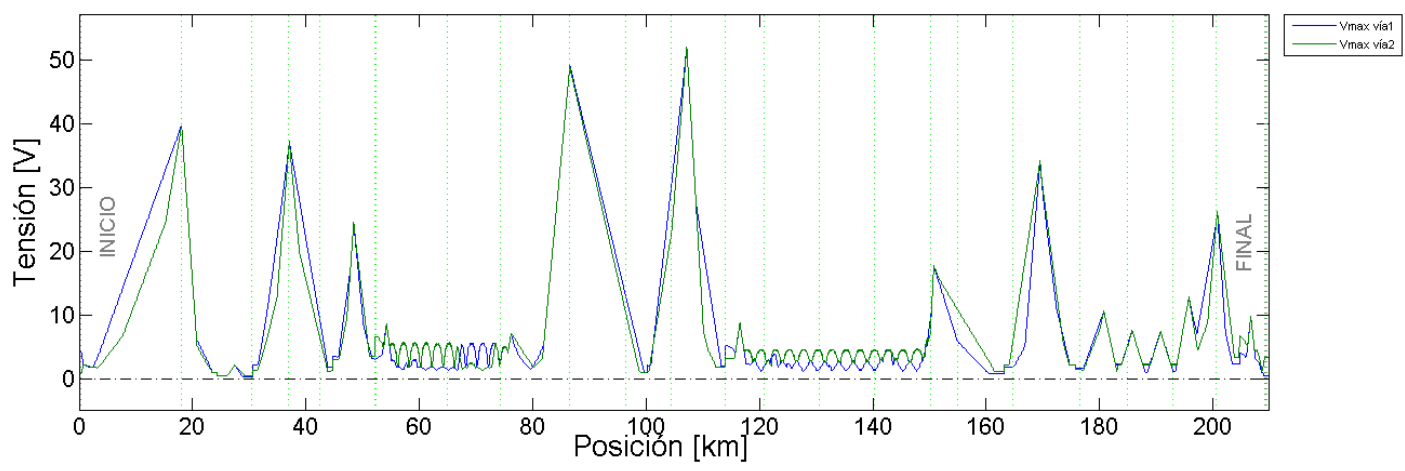

Figura 117 Tensiones máximas en retorno para dimensionamiento inicial CASO 2

Un primer análisis de los datos, se comprueba que para las tensiones extremas de catenaria no ofrece un claro diagnóstico de ajuste, por lo que es posible que una optimización de costes pueda ser llevada a cabo por la Metodología, sin causar por ello un fallo en el sistema eléctrico del nuevo dimensionamiento eléctrico propuesto. En cuanto a las tensiones de retorno, por ser un valor acondicionado a la conductancia de los carriles, ya que la puesta a tierra en alterna debe ser compensada, para evitar las sobretensiones, va a ser un aspecto secundario en el análisis. Según la norma, las tensiones de retorno en alterna deberían comenzar a estudiar el régimen permanente cuando superen los $65 \mathrm{~V}$, invalidando el sistema eléctrico cuando supere los $80 \mathrm{~V}$. Con los datos obtenidos en la simulación del escenario inicial, no se observa sobretensión, aunque la conductancia carril-tierra se podría elevar mucho más según características propias de este tipo de sistema eléctrico.

Es interesante detenerse en el análisis de las tensiones de retorno, ya que la gráfica resultante necesita una explicación para poder interpretar los datos. Esta explicación deviene de la comparación con las gráficas de tensión de retorno que se han podido observar en las pruebas realizadas en el Capítulo 5 de esta Tesis. En ese caso, los picos de tensión máxima de retorno se producían en las proximidades de las cargas, o sea de los trenes. En ese instante la corriente de fuga fluía hacia los carriles desde los trenes, con un valor de conductancia bajo [20]. En ese punto la tensión, en el retorno, es la más alta del sector eléctrico. La corriente circularía de vuelta hasta las subestaciones adyacentes. El proceso en alterna es similar, salvo que el valor de la conductancia es 
mucho mayor. Tal y como se detalla en [21] la relevancia del valor de la conductancia en sistemas DC es mucho mayor que para sistemas en AC. Además en DC se suelen aplicar valores bajos, mientras que en alterna, se aplican valores más altos. Entre otros peligros de afectación al sistema ferroviario, el más relevante para evitar es el peligro de corrosión [22] [23] del material de los carriles es menor, y se permite por tanto una corriente de fuga mayor. El análisis de la malla sigue siendo similar, existe una caída de tensión desde la carga hacia la subestación perteneciente al tramo eléctrico, pero a diferencia del caso en continua, se puede observar en algunas gráficas que la tensión máxima de retorno, es mayor en las proximidades de las subestaciones que en las cargas. Esto es debido a que el valor mostrado es un valor absoluto. La caída de tensión es mucho mayor en alterna, y pasa a ser negativa, incluso superior en valor absoluto a la inicial en el circuito eléctrico formado por el tren (inicial) y la subestación.

En resumen, se puede intentar la optimización y no solo en posicionamiento, sino en tratar de ahorrar costes. A continuación primero se hará un repaso a los resultados obtenidos del proceso de optimización, para cada algoritmo y rangos de trabajo y parámetros de estudio elegidos, para finalmente mostrar los resultados eléctricos de las dos configuraciones más significativas de cara a analizar su comportamiento.

\subsubsection{Proceso optimización CASO2. Configuración y resultados. Análisis.}

Las pruebas aplicadas a este caso han sido diseñadas de modo que los conjuntos fueran incrementando en complejidad, y por tanto, también en consumo temporal. Debido a esto último se ha pensado en un par de pruebas para cada conjunto, es decir complejidad temporal baja, complejidad temporal media y complejidad temporal alta. Lo que se pretende es llegar a conclusiones de cómo afecta el número de generaciones y población, en situaciones de sistema eléctrico en alterna, y más concretamente con este proyecto, para una envergadura más grande de proyecto y de línea ferroviaria.

\subsubsection{Espectro bajo de población y número de generaciones.}

Como antecedente se demostró que los resultados para un espectro bajo de población y generaciones no eran lo suficientemente buenos, comparados con poblaciones mayores, pero en todo caso, siempre se puede optar por una configuración de este tipo, para poder comenzar a evaluar los cambios a realizar en el dimensionamiento eléctrico. 
- $\quad$-MOEA

○ Configuración

\begin{tabular}{lcc}
\hline Población & Prueba1 & Prueba2 \\
\hline Población & 20 & 20 \\
No Eval & 150 & 150 \\
Épsilon & 5000 & 5000 \\
Prob. Cruce & 0.75 & 0.75 \\
Prob. Mutación & 0.25 & 0.25 \\
Elim. Ss & -0.3 & -0.09 \\
\hline
\end{tabular}

Tabla 53 Configuración épsilon-MOEA nivel bajo

○ Resultados

\begin{tabular}{lcccrrrc}
\hline & Fitness1 & Fitness2 & Tiempo & Rest1 & Rest2 & Rank1 & Rank2 \\
\hline Prueba1 & 24872 & 25239.8 & $89^{\prime}$ & $\mathbf{a}$ & $\mathbf{a}$ & 1 & 2 \\
Prueba2 & 35407.6 & 35392.84 & $90^{\prime}$ & $\mathbf{a}$ & $\mathbf{a}$ & 1 & 3 \\
\hline
\end{tabular}

Tabla 54 Resultados épsilon-MOEA caso bajo

Una apreciación diferente respecto de las pruebas realizadas con el sistema en corriente continua, es que la solución final con estos parámetros contienen genotipos en cuyo sistema eléctrico se detectan fallos en las restricciones del modelo. La causa principal es que en casos complejos, como este diseño eléctrico, para un número de generaciones bajas, y aun más determinante, un número bajo de individuos, puede dar lugar a este comportamiento no deseado. La advertencia de no pasar alguna restricción directamente descarta el genotipo de la elección final del diseñador.
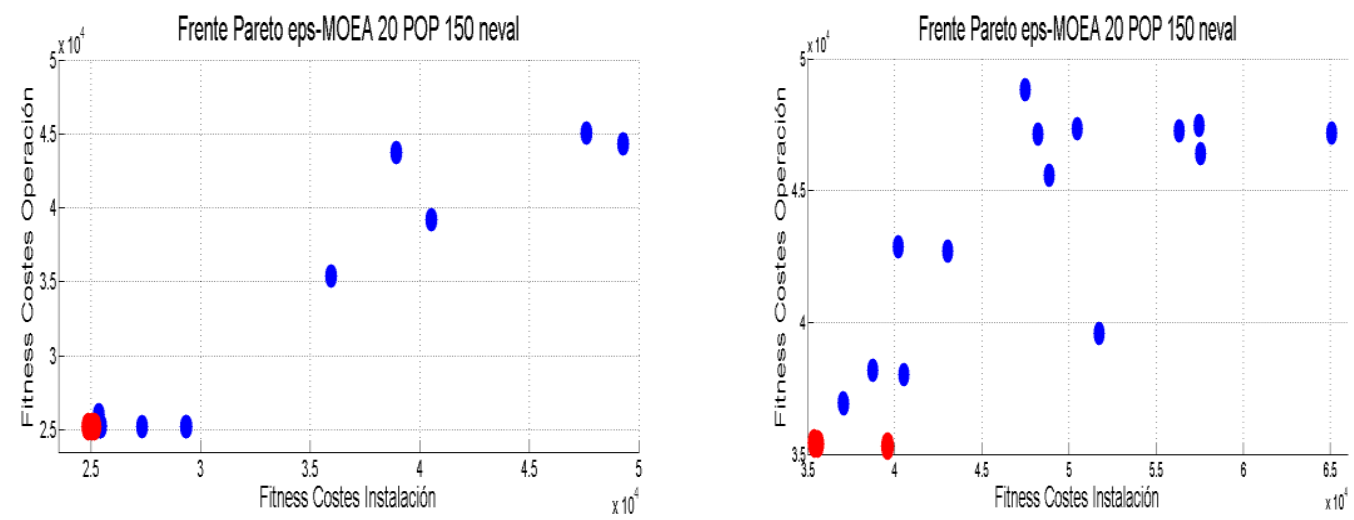

Figura 118 Frentes de Pareto. a) Prueba1;b) Prueba 2

Se aprecia que a diferencia de los casos de corriente continua, en este proyecto y para las condiciones iniciales del algoritmo de optimización, la diversidad que contiene es mucho mayor. Sin embargo lo que más llama la atención en este primer análisis de las pruebas es que para el caso en el que el algoritmo es capaz de eliminar las subestaciones con mayor probabilidad, llega a unos resultados mucho mejores que para el otro caso, llegando la diferencia a ser aproximadamente de un $50 \%$ en ambos fenotipos calculados. 
- AMGA-II

○ Configuración

\begin{tabular}{lcc}
\hline Población & Prueba1 & Prueba2 \\
\hline Población final & 20 & 20 \\
No Eval & 150 & 150 \\
Generaciones & 10 & 10 \\
Prob. Cruce & 0.8 & 0.75 \\
Prob. Mutación & 0.25 & 0.25 \\
Elim. Ss & -0.08 & -0.28 \\
\hline
\end{tabular}

Tabla 55 Configuración AMGA-II nivel bajo

Vamos a ver cómo se comporta el algoritmo AMGA-II con parámetros iniciales equivalentes a los usados en los otros algoritmos.

○ Resultados

\begin{tabular}{lccccccc}
\hline & Fitness1 & Fitness2 & Tiempo & Rest1 & Rest2 & Rank1 & Rank2 \\
\hline Prueba1 & 38512.9 & 38546.3 & $3 \mathrm{~h} \mathrm{20}$ & $\mathbf{\square}$ & $\mathbf{\square}$ & 2 & 3 \\
& 45083 & 37309.9 & & & & & \\
Prueba2 & 31137.4 & 28875.7 & $3 \mathrm{~h} 17$ & $\mathbf{\square}$ & $\mathbf{\square}$ & 2 & 4 \\
\hline
\end{tabular}

Tabla 56 Resultados AMGA-II caso bajo
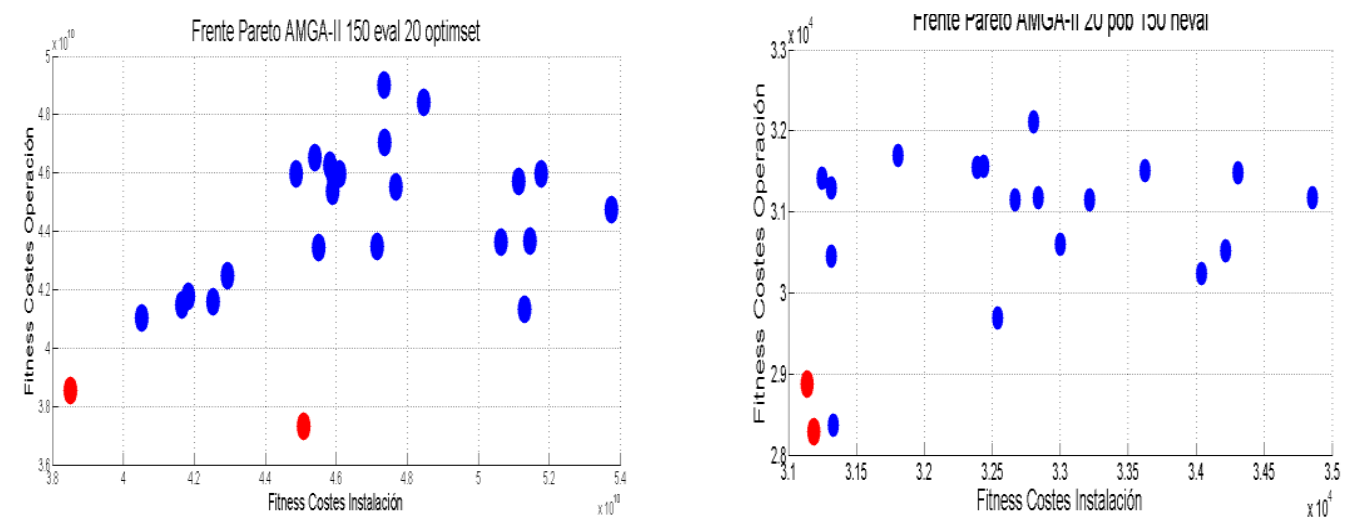

Figura 119 Frentes de Pareto. a) Prueba1;b) Prueba 2

Se repite el patrón cuando se aplica una alta probabilidad de eliminación de subestación: mejores resultados en paso final del algoritmo. En este caso vuelve a ser considerable la diferencia. Si se considera que el sistema está sobredimensionado, para instancias bajas de lanzamiento de la Metodología, está siendo siempre más eficaz usar una probabilidad elevada de eliminación de subestaciones. Por otra parte, se confirma que la diversidad del espacio de búsqueda es ampliamente mejor que para el caso comentado anteriormente de Corriente Continua.

Otro punto a destacar es el tiempo consumido en alcanzar la generación final. La conclusión a la que se llega es que se ha producido debido a un mayor número de generaciones, lo que ha hecho consumir mucho más tiempo para hallar el resultado. 
- NSGA-II

○ Configuración

\begin{tabular}{lcc}
\hline Población & Prueba1 & Prueba2 \\
\hline Población final & 16 & 16 \\
Generaciones & 8 & 8 \\
Prob. Cruce & 0.75 & 0.9 \\
Prob. Mutación & 0.2 & 0.1 \\
Elim. Ss & -0.08 & -0.3 \\
\hline
\end{tabular}

Tabla 57 Configuración NSGA-II nivel bajo

Se ha introducido una variante en las pruebas que se centra en cambiar más de un parámetro entre ellas. El valor alto de probabilidad para eliminar la subestación debería poder compensarse con valores bajos de probabilidad de cruce y mutación. En cambio en la otra prueba los valores de los operadores clásicos, son altos.

○ Resultados

\begin{tabular}{|c|c|c|c|c|c|c|c|}
\hline & Fitness 1 & Fitness2 & Tiempo & Rest1 & Rest2 & Rank1 & Rank2 \\
\hline \multirow{4}{*}{ Prueba1 } & 31922 & 29391 & 1h 40' & $\mathbf{\square}$ & $\square$ & 1 & 8 \\
\hline & 37857 & 38020 & & & & & \\
\hline & 37861 & 38019 & & & & & \\
\hline & 37855 & 38021 & & & & & \\
\hline \multirow{4}{*}{ Prueba2 } & 37878 & 38019 & 1h $41^{\prime}$ & च & [ & 7 & 2 \\
\hline & 37847 & 38113 & & & & & \\
\hline & 37880 & 38017 & & & & & \\
\hline & 37861 & 38019 & & & & & \\
\hline
\end{tabular}

Tabla 58 Resultados NSGA-II caso bajo
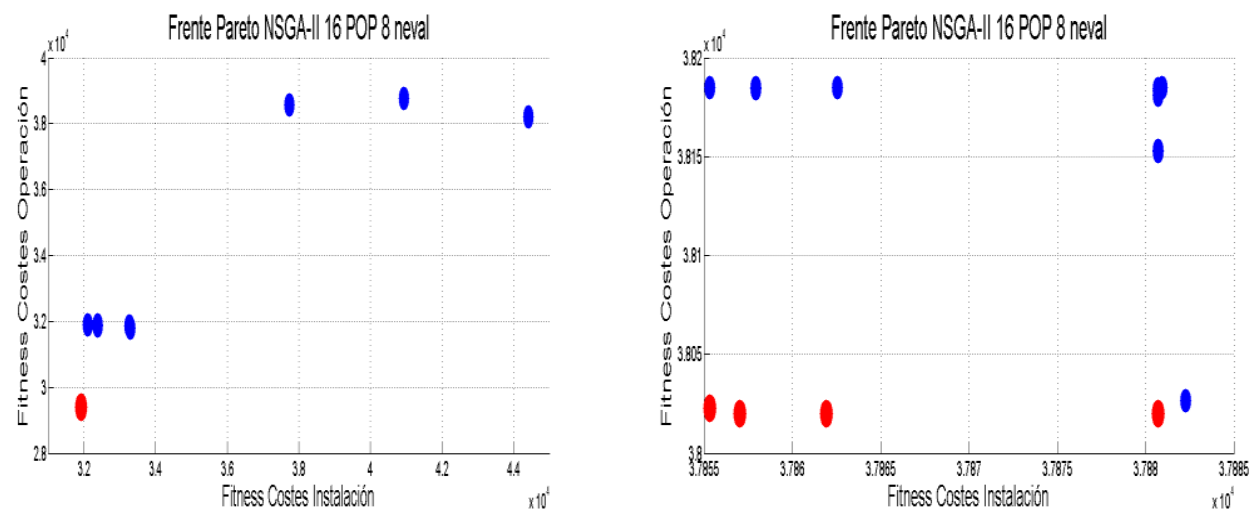

Figura 120 Frentes de Pareto. a) Prueba1;b) Prueba 2

Las consecuencias del experimento con los valores de los parámetros origina varios efectos. Uno es que la alta disparidad entre resultados ahora se invierte, el mejor lo obtiene la prueba con probabilidad baja de eliminar subestaciones. Aunque se puede decir que la diferencia no es tan amplia. En cuanto a la prueba 2 es evidente que la falta de capacidad para alterar las generaciones, con valores bajos de probabilidad para los 
operadores, ha afectado mucho a conseguir un frente más reducido, teniendo como frente de Pareto final un conjunto de 7 individuos, con valores muy parecidos entre ellos.

\subsubsection{Espectro medio de población y número de generaciones.}

Recogiendo la experiencia obtenida en los estudios previos realizados solo con el algoritmo NSGA-II, se ha establecido este nivel medio de análisis por parte de los algoritmos genéticos.

- $\quad$-MOEA

○ Configuración

\begin{tabular}{lcc}
\hline Población & Prueba1 & Prueba2 \\
\hline Población & 50 & 40 \\
No Eval & 300 & 350 \\
Épsilon & 2500 & 5500 \\
Prob. Cruce & 0.75 & 0.75 \\
Prob. Mutación & 0.25 & 0.25 \\
Elim. Ss & -0.09 & -0.3 \\
\hline
\end{tabular}

Tabla 59 Configuración épsilon-MOEA nivel intermedio

Se siguen manteniendo los valores de probabilidad de aplicar operadores.

○ Resultados

\begin{tabular}{lccccccc}
\hline & Fitness1 & Fitness2 & Tiempo & Rest1 & Rest2 & Rank1 & Rank2 \\
\hline Prueba1 & 27094.7 & 27136.4 & $3 \mathrm{~h}$ & $\mathbf{0}$ & $\mathbf{\square}$ & 5 & 5 \\
Prueba2 & 24433.2 & 25270.7 & $3 \mathrm{~h} 21$ & $\mathbf{\square}$ & $\mathbf{\square}$ & 3 & 4 \\
\hline
\end{tabular}

Tabla 60 Resultados épsilon-MOEA caso intermedio

Los resultados del frente de Pareto para las 5 son muy parecidos, por este motivo no se muestran ya que no significan mucha más información relevante. Para la prueba 2 si que hay un poco más de relevancia entre dos fenotipos en concreto. Son los que se han representado en la tabla. 

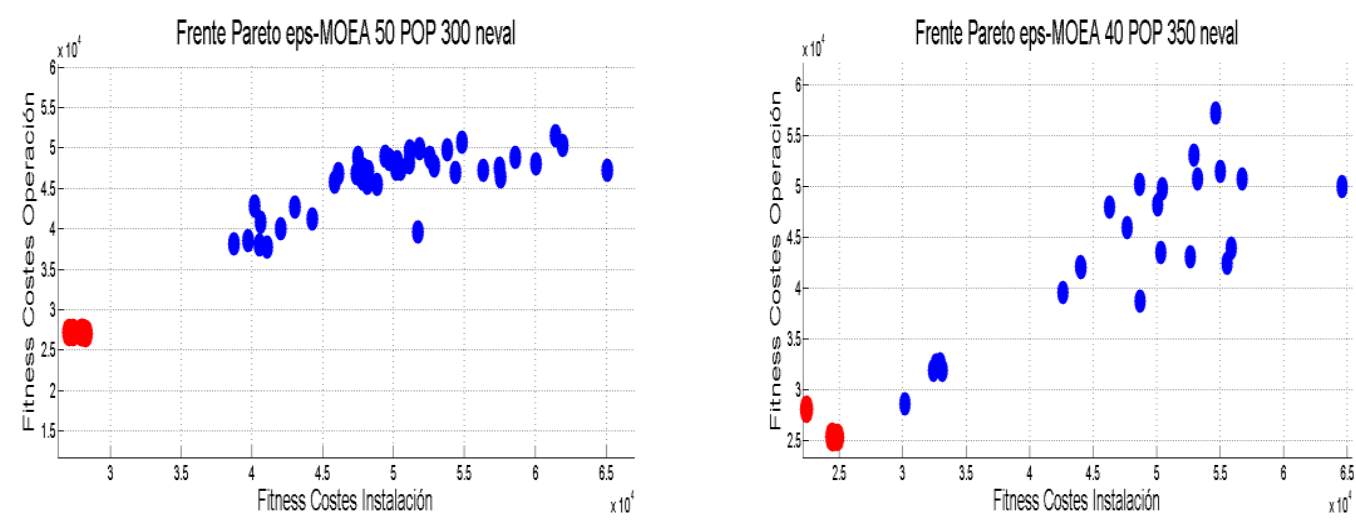

Figura 121 Frentes de Pareto. a) Prueba1;b) Prueba 2

Como se verá en los últimos ejemplos con más alta cantidad de población y generaciones, estos valores se acercan mucho más a los definitivos. Se puede decir que debido al alto valor de épsilon, se está separando claramente entre los valores mejores de cada generación del resto, como queda demostrado en ambos ejemplos.

- AMGA-II

○ Configuración

\begin{tabular}{lcc}
\hline Población & Prueba1 & Prueba2 \\
\hline Población final & 26 & 26 \\
$\mathrm{~N}^{0}$ Eval & 250 & 250 \\
Generaciones & 26 & 26 \\
Prob. Cruce & 0.8 & 0.8 \\
Prob. Mutación & 0.15 & 0.15 \\
Elim. Ss & -0.28 & -0.08 \\
\hline
\end{tabular}

Tabla 61 Configuración AMGA-II caso intermedio

Se sigue con el mismo criterio de ir comparando poblaciones similares atendiendo a los parámetros de límite de eliminación de subestación y la probabilidad de cruce, como mayor variación entre las distintas pruebas.

○ Resultados

\begin{tabular}{lccccccc}
\hline & Fitness1 & Fitness2 & Tiempo & Rest1 & Rest2 & Rank1 & Rank2 \\
\hline Prueba1 & 40021.5 & 40979.3 & 4 h 42 & 口 & 口 & 1 & 3 \\
Prueba2 & 27906 & 28883 & 4h 42 & $\mathbf{\square}$ & $\mathbf{\square}$ & 2 & 3 \\
\hline
\end{tabular}

Tabla 62 Resultados AMGA-II caso intermedio 

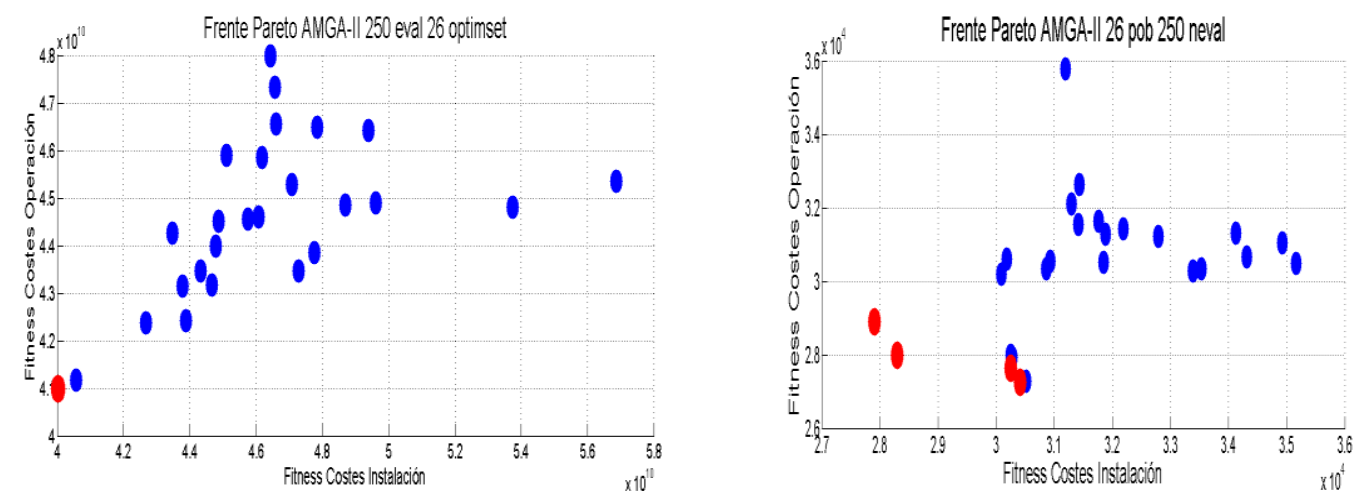

Figura 122 Frentes de Pareto. a) Prueba1;b) Prueba 1

Los resultados de la pruebal muestran una disparidad muy elevada con los resultados mejores obtenidos hasta el momento. Esto es debido a la poca probabilidad de eliminar subestaciones, por lo que deja poco margen para mejorar los costes, y a una población inicial aleatoria con muy altos costes. La segunda prueba se acerca mucho más a lo esperado, con un Frente de Pareto conformado por dos conjuntos de 2 genotipos distintos, y en valores fitness muy próximos al óptimo.

\section{- NSGA-II}

○ Configuración

\begin{tabular}{lcc}
\hline Población & Prueba1 & Prueba2 \\
\hline Población final & 72 & 72 \\
Generaciones & 16 & 16 \\
Prob. Cruce & 0.7 & 0.9 \\
Prob. Mutación & 0.25 & 0.1 \\
\hline Elim. Ss & -0.09 & -0.3 \\
\hline
\end{tabular}

Tabla 63 Configuración NSGA-II caso intermedio

En esta prueba aumenta significativamente la población y el número de generaciones.

○ Resultados

\begin{tabular}{|c|c|c|c|c|c|c|c|}
\hline & Fitness 1 & Fitness 2 & Tiempo & Rest1 & Rest2 & Rank1 & Rank2 \\
\hline \multirow{3}{*}{ Prueba1 } & 28864 & 27117 & \multirow{2}{*}{ 7h 38' } & \multirow{3}{*}{ [ } & \multirow{3}{*}{ 口 } & \multirow{3}{*}{15} & \multirow{3}{*}{22} \\
\hline & 26534 & 27963.1 & & & & & \\
\hline & 27305.8 & 28385 & & & & & \\
\hline \multirow{2}{*}{ Prueba2 } & 27214 & 28442.2 & \multirow[t]{2}{*}{$7 \mathrm{~h} 25^{\prime}$} & \multirow[t]{2}{*}{ घ } & \multirow[t]{2}{*}{$\mathbf{\square}$} & \multirow[t]{2}{*}{21} & \multirow[t]{2}{*}{26} \\
\hline & 27618.9 & 27492.3 & & & & & \\
\hline
\end{tabular}

Tabla 64 Resultados NSGA-II caso intermedio 

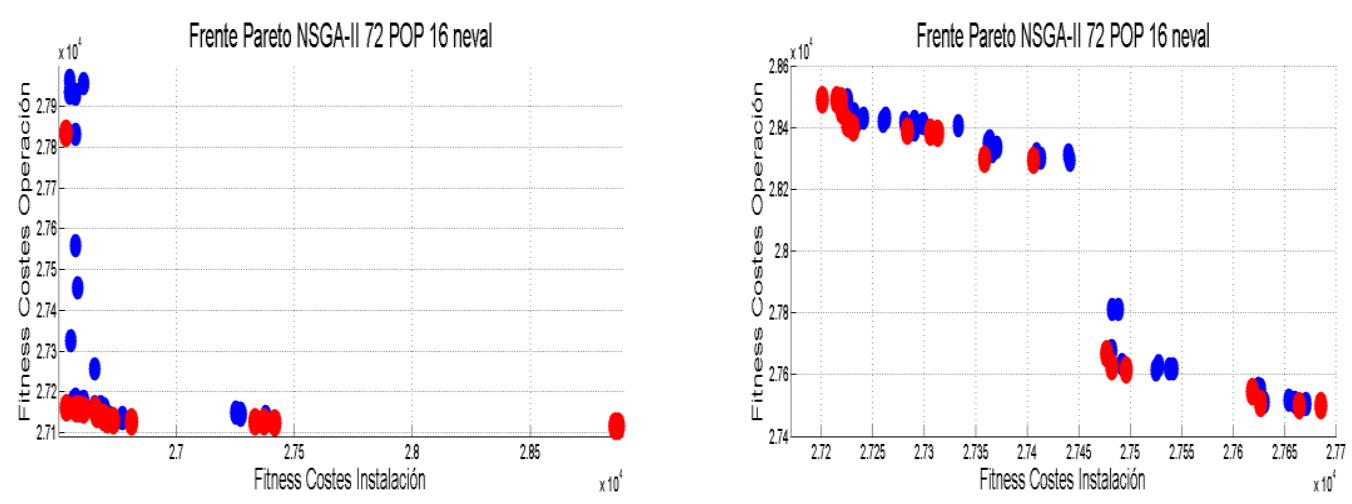

Figura 123 Frentes de Pareto. a) Prueba1;b) Prueba2

Destaca en esta prueba el alto número de integrantes del Frente de Pareto final. Existe una gran diversidad que provoca que haya muchos puntos óptimos encontrados, en términos de dominancia clásica de unos fenotipos sobre otros. Este suceso en un algoritmo como el épsilon-moea si se escoge correctamente el valor del épsilon no debería ocurrir y las élites estarían mucho más separadas del resto de niveles de valoración. A tener en cuenta también el coste temporal, mucho más alto de nuevo que para los otros algoritmos.

\subsubsection{Espectro alto de población y número de generaciones.}

Los tiempos de espera son muy importantes, y para un nivel alto de población y generaciones se debe considerar el beneficio que se va a obtener respecto de la penalización de la complejidad temporal añadida. Por este motivo se van a mostrar los resultados obtenidos y tener una herramienta más para poder determinar si es práctico o no, realizar optimizaciones con este número elevado de participantes. .

- $\quad$-MOEA

○ Configuración

\begin{tabular}{lcc}
\hline Población & Prueba1 & Prueba2 \\
\hline Población & 100 & 100 \\
N$^{0}$ Eval & 700 & 700 \\
Épsilon & 3000 & 1000 \\
Prob. Cruce & 0.9 & 0.75 \\
Prob. Mutación & 0.1 & 0.25 \\
Elim. Ss & -0.3 & -0.09 \\
\hline
\end{tabular}

Tabla 65 Configuración épsilon-MOEA nivel alto

○ Resultados

\begin{tabular}{lccccccc}
\hline & Fitness1 & Fitness2 & Tiempo & Rest1 & Rest2 & Rank1 & Rank2 \\
\hline Prueba1 & 24990 & 25146.5 & $8 \mathrm{~h} \mathrm{18}$ & $\mathbf{0}$ & $\mathbf{0}$ & 4 & 5 \\
Prueba2 & 26519.8 & 26936.2 & $7 \mathrm{~h} \mathrm{58}$ & $\mathbf{\square}$ & $\mathbf{\square}$ & 1 & 2 \\
\hline
\end{tabular}



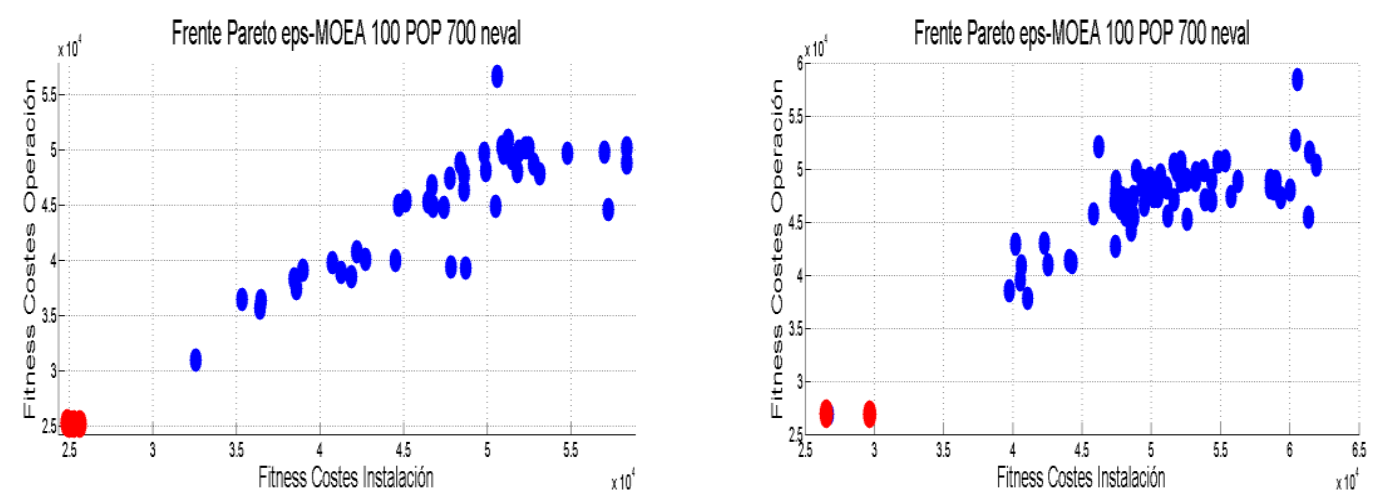

Figura 124 Frente de Pareto a)Prueba1;b)Prueba2

Se sigue manifestando en los resultados finales el funcionamiento de este algoritmo. Mucha diversidad y separación entre los genotipos del frente de Pareto respecto de los demás. En cuanto a los fenotipos no son mucho mejores que los anteriores, por lo que se puede decir que no es necesario, en un problema de este tipo, llegar a evaluar con más rango de población.

- AMGA-II

○ Configuración

\begin{tabular}{lcc}
\hline Población & Prueba1 & Prueba2 \\
\hline Población final & 150 & 150 \\
No Eval & 1500 & 1500 \\
Generaciones & 152 & 152 \\
Prob. Cruce & 0.8 & 0.75 \\
Prob. Mutación & 0.2 & 0.2 \\
Elim. Ss & -0.08 & -0.28 \\
\hline
\end{tabular}

Tabla 67 Configuración AMGA-II caso alto

Por último, se trata de buscar un amplio margen del espacio de soluciones para ver el comportamiento del algoritmo. Modificaciones como siempre en probabilidad de cruce y eliminación de subestación.

- Resultados

\begin{tabular}{cccccccc}
\hline & Fitness1 & Fitness2 & Tiempo & Rest1 & Rest2 & Rank1 & Rank2 \\
\hline Prueba1 & 30091 & 29881.1 & $8 \mathrm{~h} \mathrm{50}$ & $\mathbf{0}$ & $\mathbf{0}$ & 1 & 5 \\
& 24840.2 & 26087.2 & & & & & \\
Prueba2 & 25241.1 & 25263.8 & 9h 10, & $\mathbf{\square}$ & $\mathbf{0}$ & 3 & 2 \\
& 26177 & 25176.8 & & & & & \\
\hline
\end{tabular}

Tabla 68 Resultados AMGA-II caso alto 

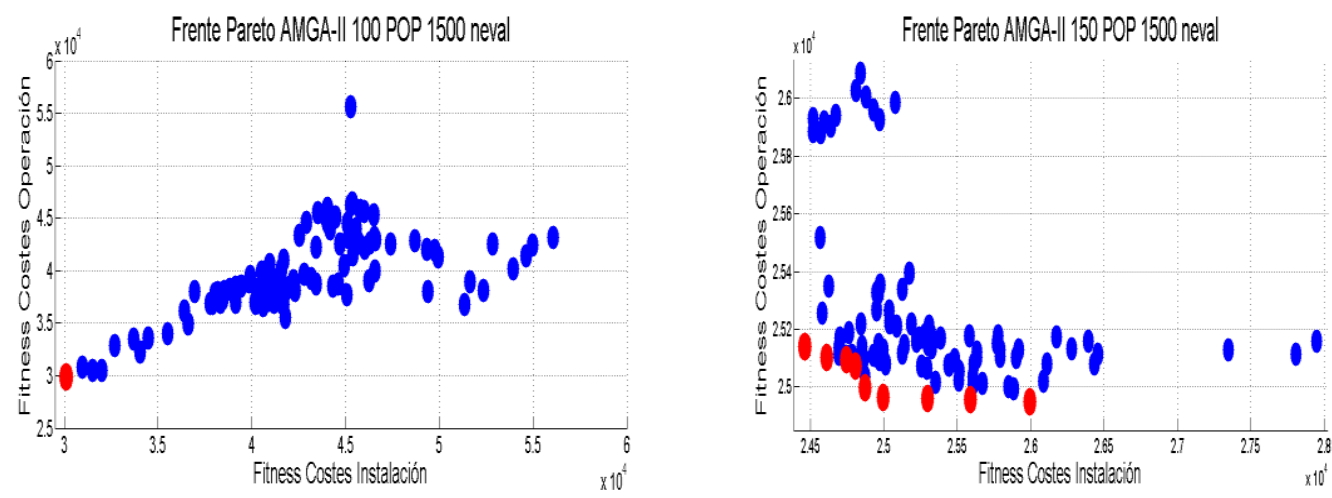

Figura 125 Frentes de Pareto. a) Prueba1;b) Prueba 2

De nuevo se demuestra que para este sistema eléctrico, inicialmente sobredimensionado, el parámetro más influyente para conseguir un mejor resultado es la probabilidad de eliminar subestaciones. Esto hace que se halle de forma más segura una configuración sin las subestaciones que se consideren, tal y como se ve en la gráfica de la prueba2. Además con altos valores para operadores de cruce y mutación se obtienen frentes con diversidad y, en definitiva, un frente de Pareto mucho más relevante que para el de prueba1.

\section{- NSGA-II}

○ Configuración

\begin{tabular}{lc}
\hline Población & Prueba1 \\
\hline Población final & 130 \\
Generaciones & 20 \\
Prob. Cruce & 0.7 \\
Prob. Mutación & 0.2 \\
Elim. Ss & -0.3 \\
\hline
\end{tabular}

Tabla 69 Configuración NSGA-II nivel alto

La prueba trata de conseguir resultados ya con un valor de probabilidad de eliminación de subestación muy alto, y con los valores de operadores de cruce y mutación también altos.

○ Resultados

\begin{tabular}{cccccccc}
\hline & Fitness1 & Fitness2 & Tiempo & Rest1 & Rest2 & Rank1 & Rank2 \\
\hline \multirow{2}{*}{ Prueba1 } & 24412.1 & 25155.2 & $18 \mathrm{~h} 40$ & $\mathbf{0}$ & $\mathbf{0}$ & 8 & 14 \\
\hline
\end{tabular}




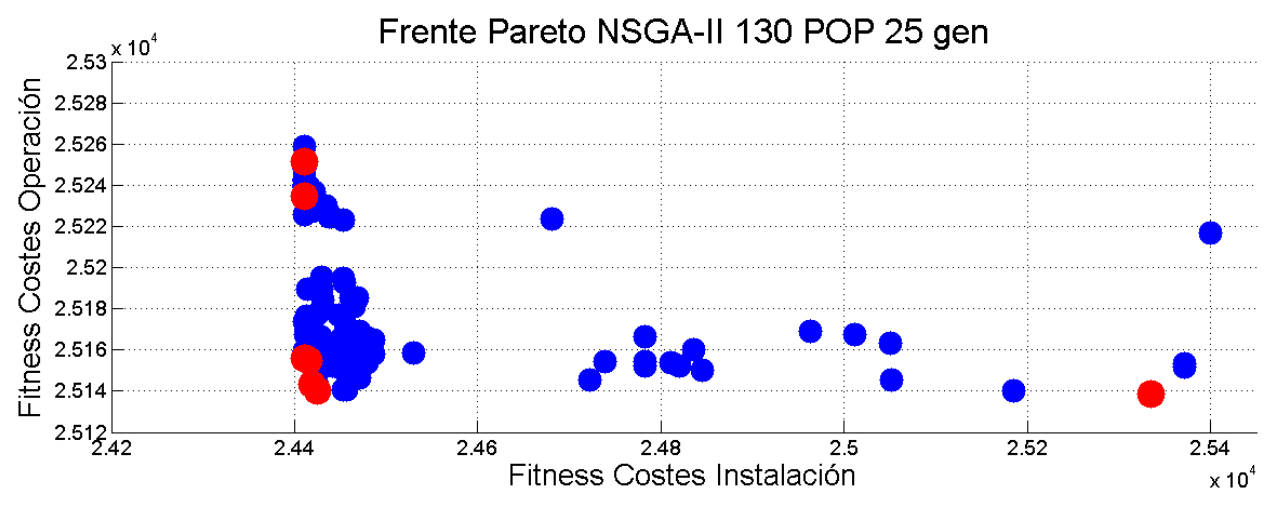

Figura 126 Frentes de Pareto de la Prueba1

De nuevo se tiene un resultado mucho más homogéneo que para las otras alternativas de algoritmos genéticos. Esto provoca que la movilidad de los genotipos sea mucho menor, ya que cualquier alteración genética se resolverá entre individuos con características muy similares, por lo que el recorrido de la población a lo largo de las generaciones, muy difícilmente será variado, tal y como se ha podido comprobar en nuestros experimentos. De nuevo el tiempo consumido es muy elevado, aunque finalmente, se obtienen también resultados satisfactorios en cuanto a encontrar un dimensionamiento eléctrico más ajustado a las necesidades reales..

\subsubsection{Conclusiones.}

Lo que más destaca es que para realizar una optimización para un proyecto de este tipo, no es necesario iniciar con una población muy alta, ni con muchas generaciones de evaluación genética de los componentes. Con la configuración media, en general, se han obtenido buenos resultados y muy cercanos a lo que se pretendía, que es mejorar el diseño inicial con un aceptable valor en coste, tanto para la función objetivo de instalación como para la de los costes de operación, añadiendo además que se puede elegir entre sistemas eléctrico en un tiempo mucho menor que si se hubiera trabajado con la Metodología tradicional.

Como se preveía, y siempre en comparación con el CASO 2 de corriente continua, en este caso, para todos los ejemplos se observa una diversidad que no existía en el otro. Esto demuestra que el algoritmo es capaz de hallar soluciones en un amplio rango del espacio de posibilidades, y de que la complejidad del método, en cuanto a examinar múltiples casos, da al ingeniero una herramienta capaz de analizar diferentes sistemas eléctricos, teniendo en cuenta las consecuencias de cada uno, para que finalmente se pueda escoger una variante examinada y resuelta.

En cuanto a las diferencias entre los algoritmos, es importante destacar que los tiempos de resolución del NSGA-II son mucho más altos que para los otros dos. Si se analizan los resultados finales no se observa una mejora que canalice ese tiempo de más en una solución mejor. Por lo que de nuevo, para este tipo de proyectos, con los parámetros y genotipos de este tamaño de bits, y sobre todo, con el objetivo final, que no es otro que hallar un dimensionamiento mejorado, la mejor opción siempre podrá ser 
el algoritmo épsilon-moea o el amga-ii, ya que en términos temporales son mucho mejores.

\subsubsection{Elección 1 dimensionamiento eléctrico con 4 subestaciones de tracción y 12 autotransformadores.}

Este primer ejemplo, más conservador que el que se mostrará posteriormente, dictamina que se puede eliminar una subestación de tracción del diseño inicial. Esto en costes, evidencia un ahorro considerable. En cuanto a la catenaria, sigue quedándose con la catenaria original en prácticamente todo el recorrido.

En los resultados se mostrarán las tensiones mínimas en pantógrafo para cada uno de los escenarios posibles más relevante de cara a examinar la validez del nuevo dimensionamiento eléctrico.

Para elegir el escenario de los casos presentados por los algoritmos con la característica principal de eliminar una subestación, se ha cogido el que tenía mejores costes de operación.

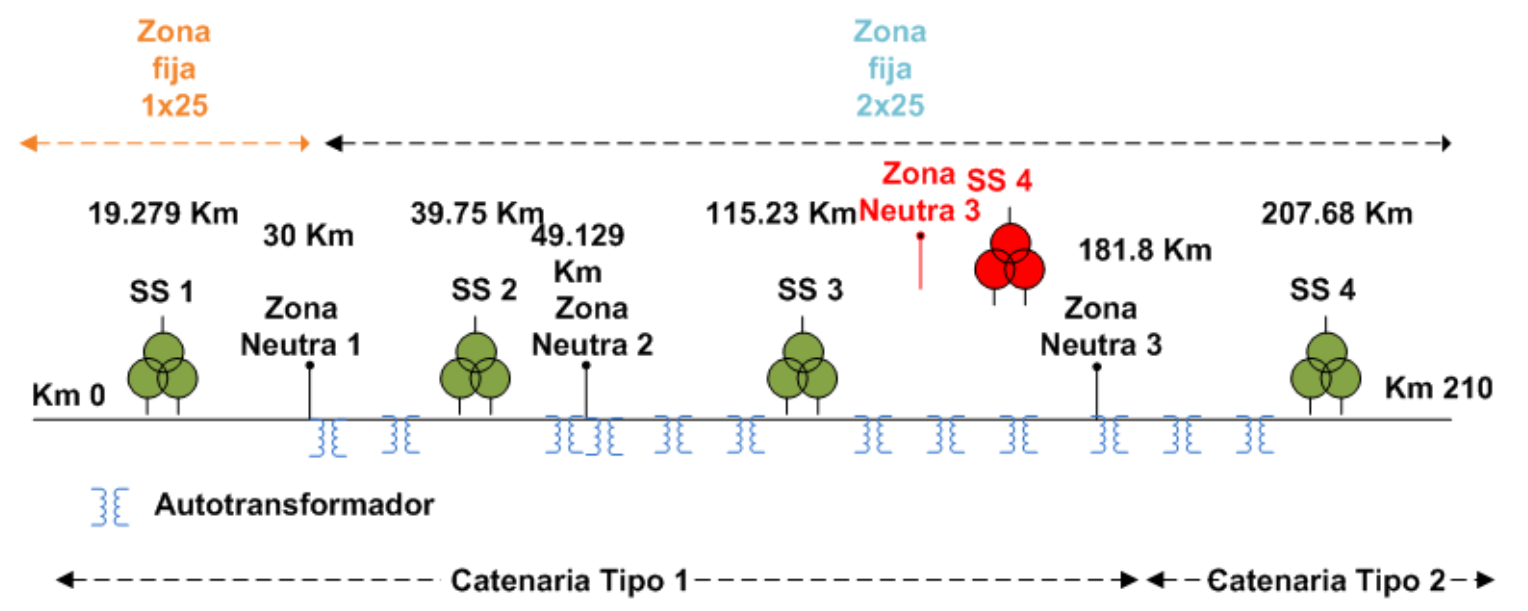

Figura 127 Escenario sistema eléctrico caso 4 subestaciones de tracción mejores costes de operación

Un tema muy importante es el posicionamiento de los elementos eléctricos y saber en qué medida han podido evitar aquellas zonas críticas de las que se hablaba en la introducción. Hay que decir que si bien las subestaciones todas han evitado alguna zona crítica de gran impacto, debido a la gran cantidad de autotransformadores y a la elevada discretización zonal, ha habido un autotransformador que ha sido instalado en una de esas zonas, en concreto en el pk 95.100, violando la zona medioambiental crítica. En el análisis de la mejor opción para costes de instalación se comprobará si a pesar de la elevada construcción de elementos eléctricos que son necesarios, es posible no intersectar con una zona de elevado valor, en este caso ecológico. 


\section{- Funcionamiento normal}

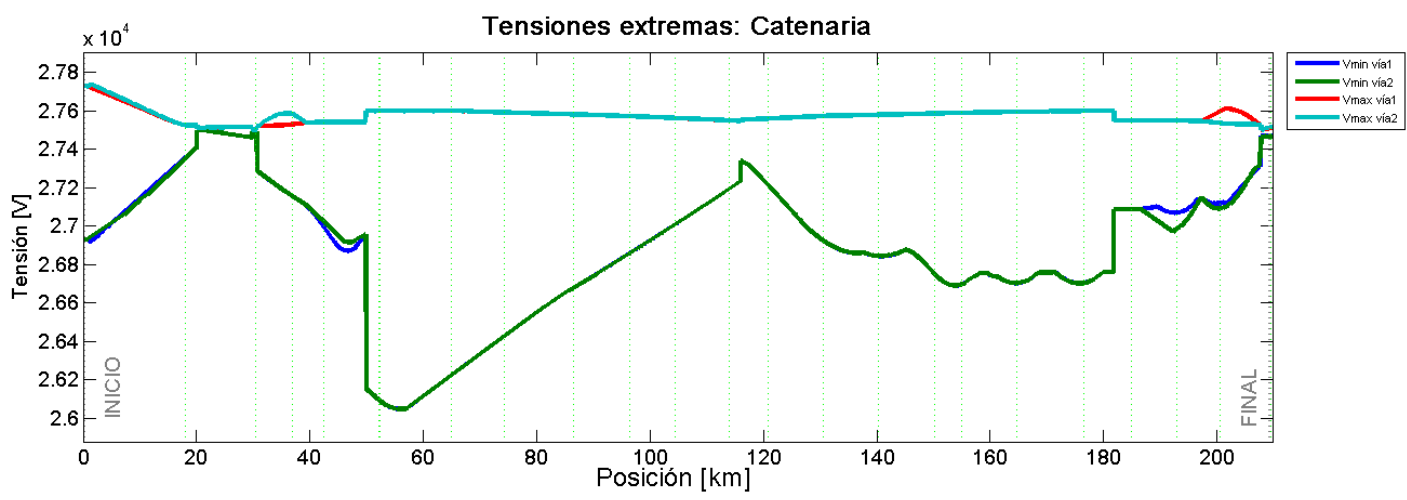

Figura 128 Tensiones extremas funcionamiento normal ejemplo 1 caso 2x25

Claramente se observa que la mayor caída en la zona posterior a la zona neutra que separa las subestaciones 1 y 2 de la zona de $2 \times 25$. En todo caso, los niveles de tensión están muy por encima del límite de los $19000 \mathrm{~V}$, situándose en los $26000 \mathrm{~V}$. Por este motivo, es muy probable que las situaciones degradadas no tengan mayores inconvenientes en cuanto a superar límites de tensión. A continuación, como se ha venido haciendo hasta ahora, se muestran los resultados obtenidos para las situaciones de fallo, y de esta manera comprobar si los test realizados durante el proceso de optimización, marcados por la Metodología explicada en el Capítulo 5, efectivamente hacen el filtro correctamente y permiten conocer situaciones adecuadas para dimensionamientos eléctricos.

\section{- Situaciones degradada simple, o caída de un grupo en la subestación}

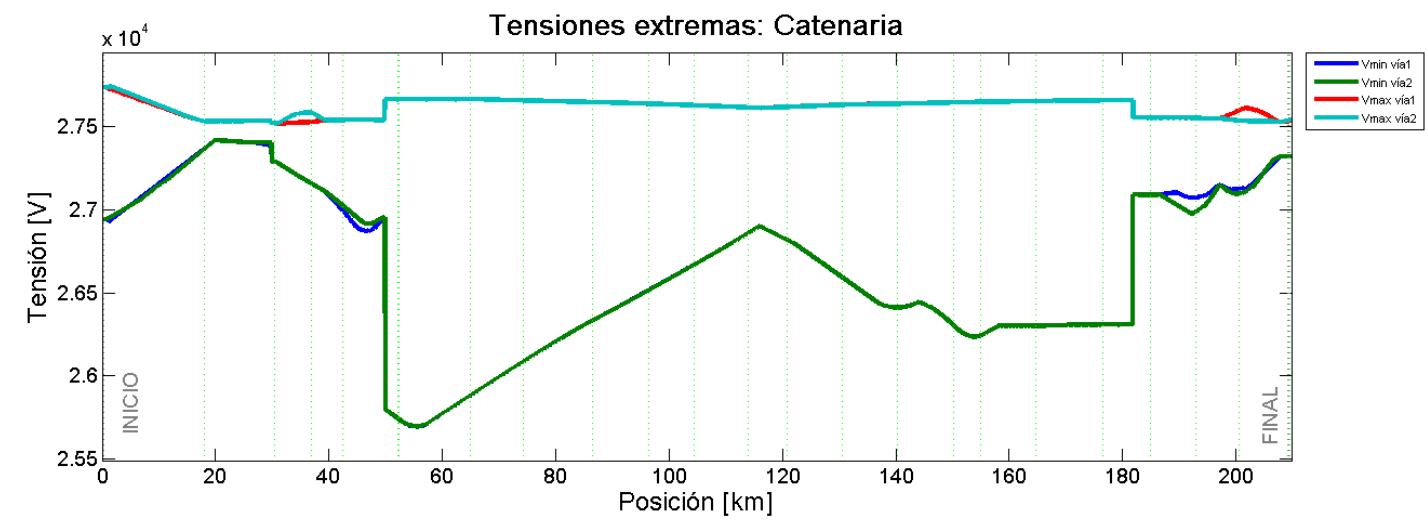

Figura 129 Degradada simple ejemplo 1 alterna $2 \times 25$

Comparando directamente con la gráfica anterior, existe una previsible mayor caída de tensión pero siempre dentro de los límites permitidos. 

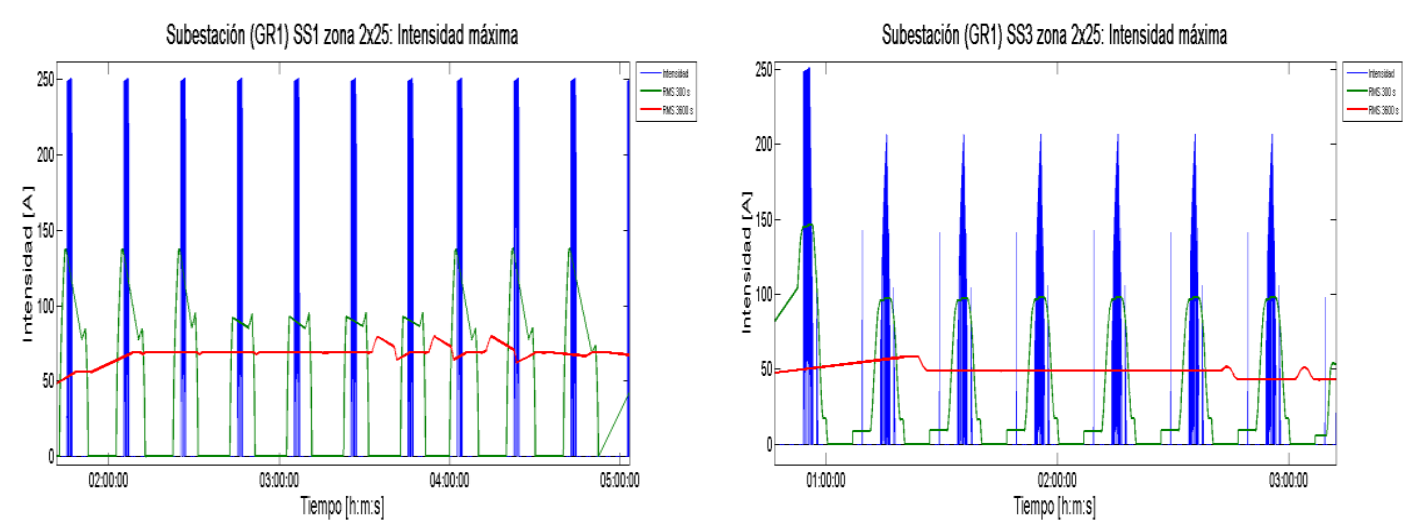

Figura 130 Intensidades máximas en a)Grupo 1 de subestación 1 zona 2x25;b)Grupo 3 zona 2x25

Para evaluar la capacidad de las subestaciones para soportar la potencia demandada, se va a proponer en estos dos ejemplos, las intensidades máximas y medias cuadráticas de las subestaciones. En este caso, como se ve en las gráficas, las intensidades están muy lejos del límite de la subestación, teniendo en cuenta los $25 \mathrm{kV}$ de tensión nominal de la línea, por lo que deducimos que no están en peligro de sobrecarga.

A continuación se simulan las situaciones de fallo de las subestaciones posibles para este dimensionamiento eléctrico.

- Situación degradada doble, caída subestación zona mono 1x25

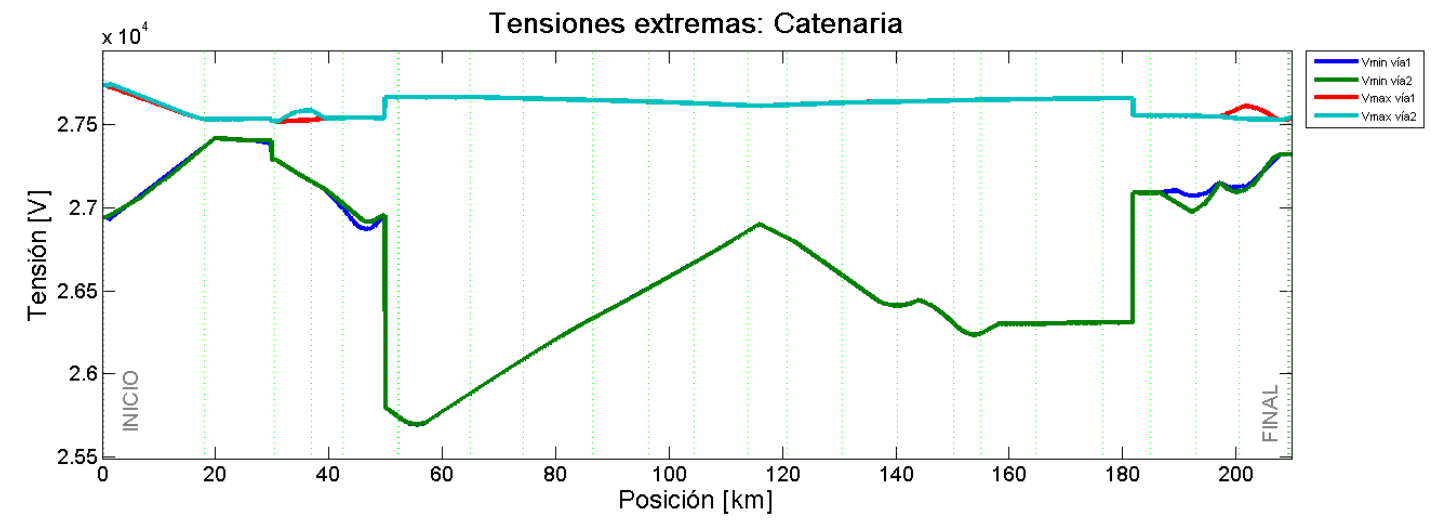

Figura 131 Situación degradada simple fallo de un transformador en cada subestación

Para esta línea con el plan de explotación diseñado de tal manera que es el mismo tanto para situación de funcionamiento normal como degradado doble, la situación degradada simple no debía ser la situación más complicada a la que se podría enfrentar el sistema eléctrico. Como se puede ver en la gráfica, las tensiones caen un poco en relación con el funcionamiento normal, pero muy alejado del valor límite.

Además de las caídas de tensión, o tensiones mínimas en catenaria, se van a estudiar el impacto de los fallos de las subestaciones calculando las intensidades en subestación, tanto las máximas como las medias cuadráticas para 5 minutos como para 1 hora de funcionamiento. 

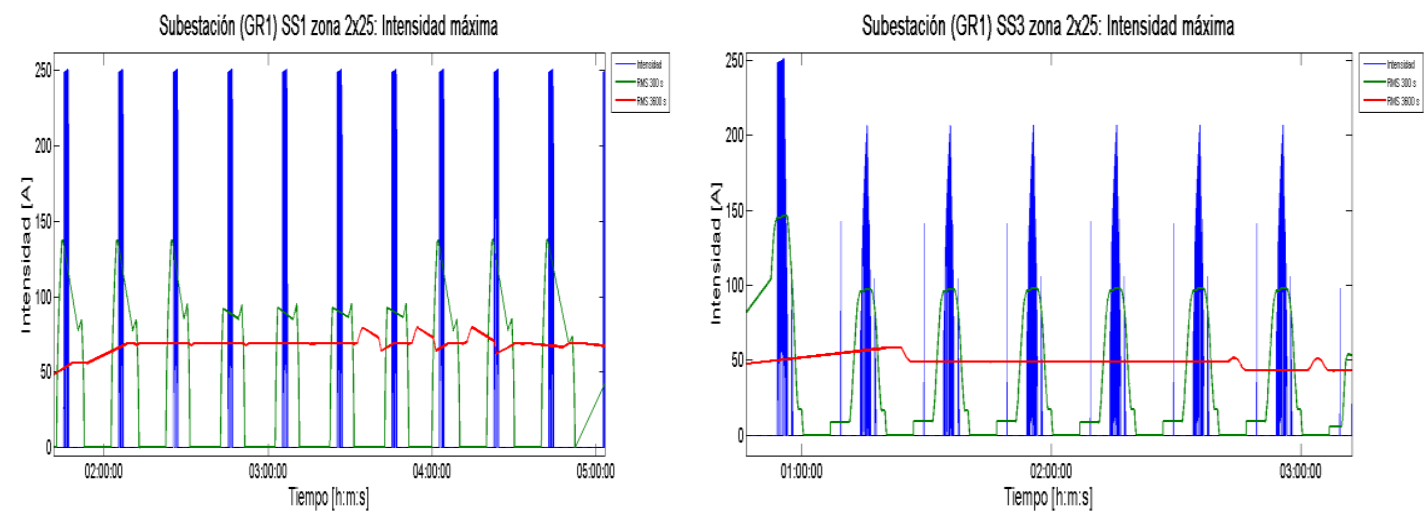

Figura 132 Intensidades máximas en a) Grupo 1 SS1 zona 2×25;b)Grupo 1 SS3 zona 2x25

De nuevo se buscan posibles sobrecalentamientos en las subestaciones. A tenor de las Intensidades rms que se pueden ver en las gráficas anteriores, los valores están muy por debajo del límite marcado por la Intensidad nominal para los grupos de transformación de las subestaciones, por lo que no habría peligro.

- Situación degradada doble, caída subestación 1 zona bitensión 2x25

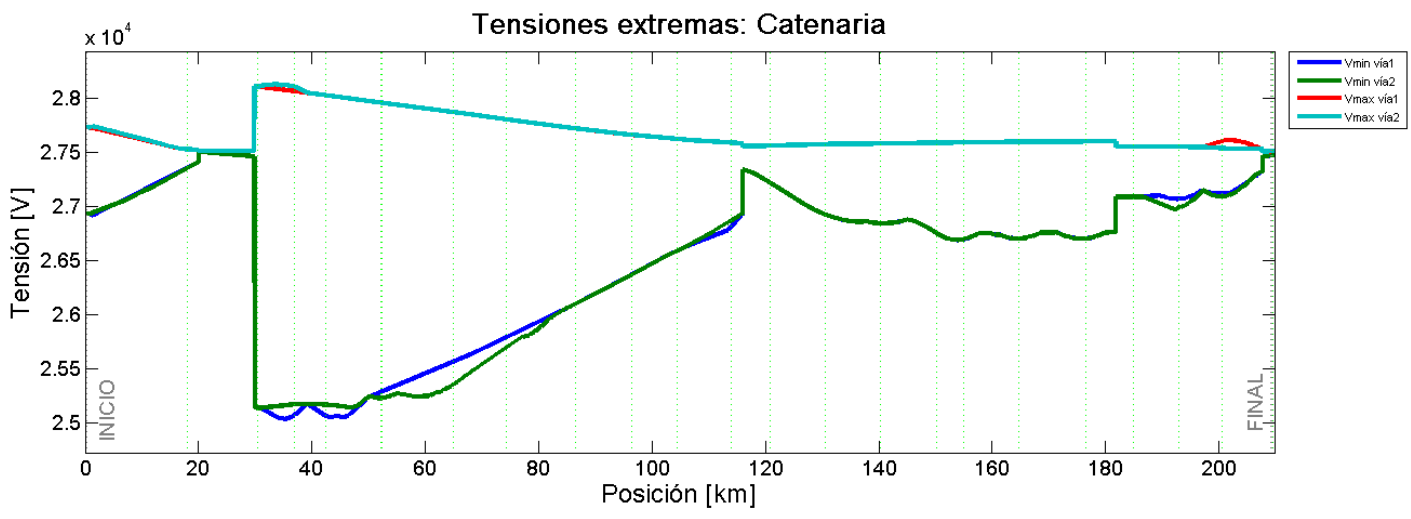

Figura 133 Tensiones extremas situación degradada completa SS1 zona 2×25
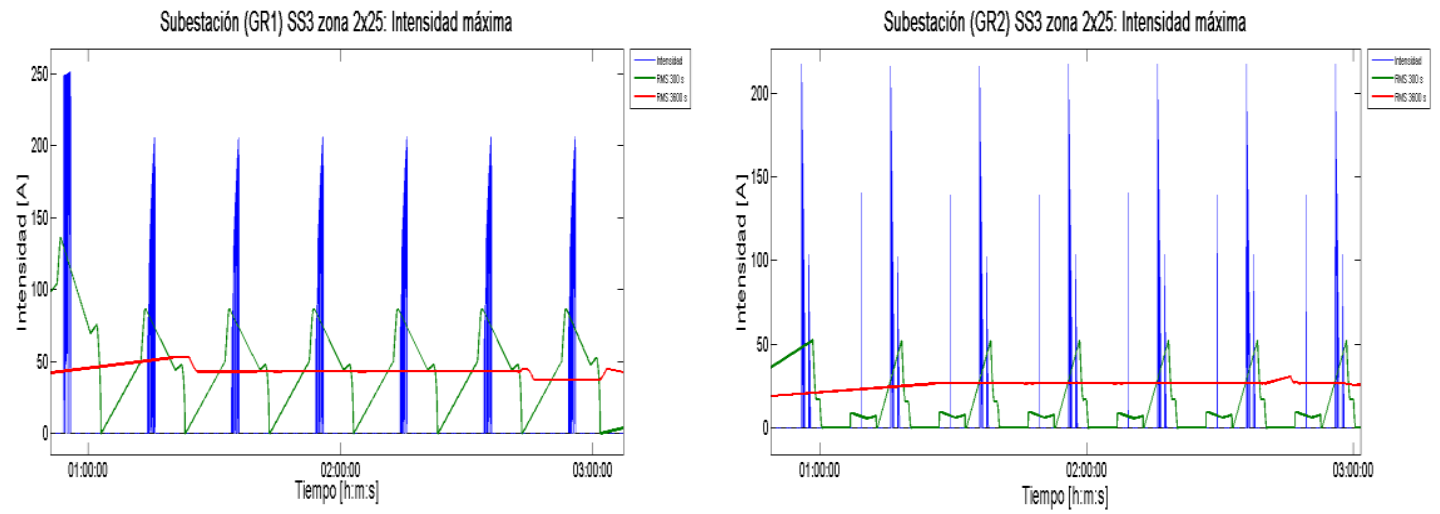

Figura 134 Intensidades máximas en a)Grupo 1 SS3 zona 2x25;b) Grupo 2 SS3 zona 2x25

El fallo doble de la subestación 1 de la zona 2x25 no representa muchos problemas en términos de caída de tensión $(25 \mathrm{kV})$ ni en posibles sobrecargas en las subestaciones 
como se puede ver en las gráficas ejemplo. En estas últimas se representan las más significativas para cada caso.

- Situación degradada doble, caída subestación 2 zona dual 2x25

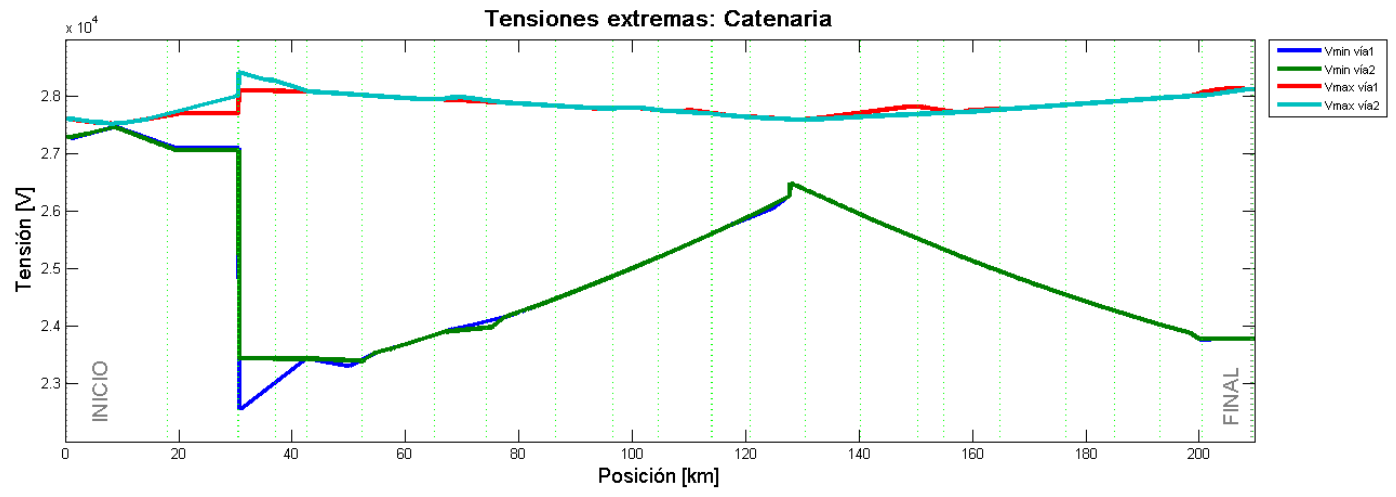

Figura 135 Tensiones extremas situación degradada en subestación 2 de zona 2×25

Esta es la situación más cercana al límite a la que se va a someter a este sistema eléctrico. La caída de tensión llega a niveles cercanos a los $22,5 \mathrm{kV}$, debido a la distancia entre la subestación en fallo y la adyacente que se ha elegido para alimentar a la zona eléctrica afectada. En todo caso, lejos del límite de los $19 \mathrm{kV}$.
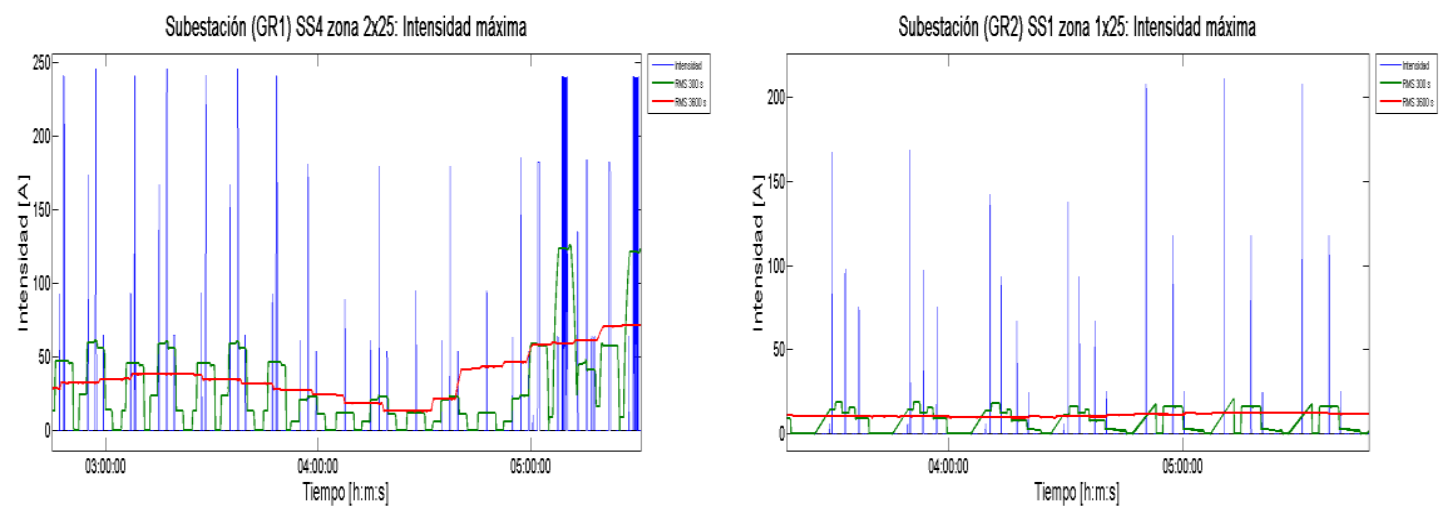

Figura 136 Intensidad máxima en a)Grupo 1 de SS4 de zona 2×25;b)Grupo 2 de SS1 zona 1×25

Las subestaciones, según los datos de las intensidades máximas y medias cuadráticas, estarían lejos de estar en sobrecarga para esta situación, tal y como se observa en los resultados significados en las gráficas anteriores. 
- Situación degradada doble, caída subestación 3 zona dual 2x25

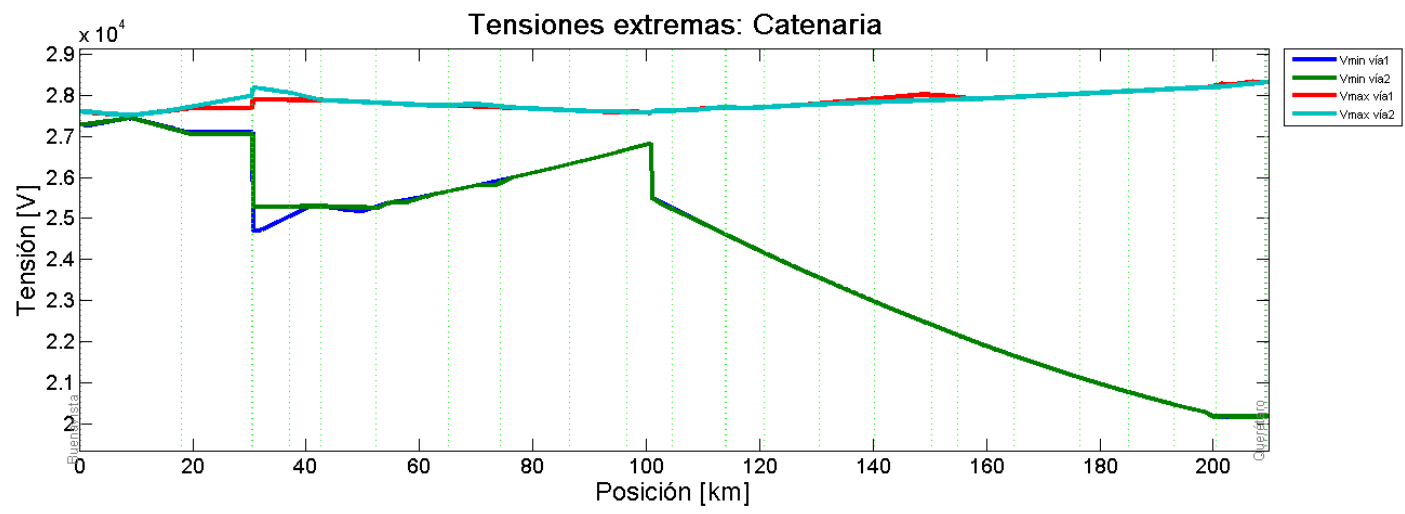

Figura 137 Tensiones extremas en situación degradada doble subestación 3 de zona 2x25

Finalmente, la situación degradada más compleja se encuentra en el fallo doble de la última subestación de la zona de $2 \times 25$. Además de tener que suministrar potencia a una longitud muy grande, y a pesar de los autotransformadores instalados, la tensión cae hasta los $20 \mathrm{kV}$, en este caso muy cerca de los $19 \mathrm{kV}$. También influyen los cambios de sentido y sobre todo los arranques de estación terminal, que necesitan más potencia que para cuando circulan en velocidad máxima.

De todos modos sigue dentro de los límites, así que seguiría validándose el sistema eléctrico en estudio.
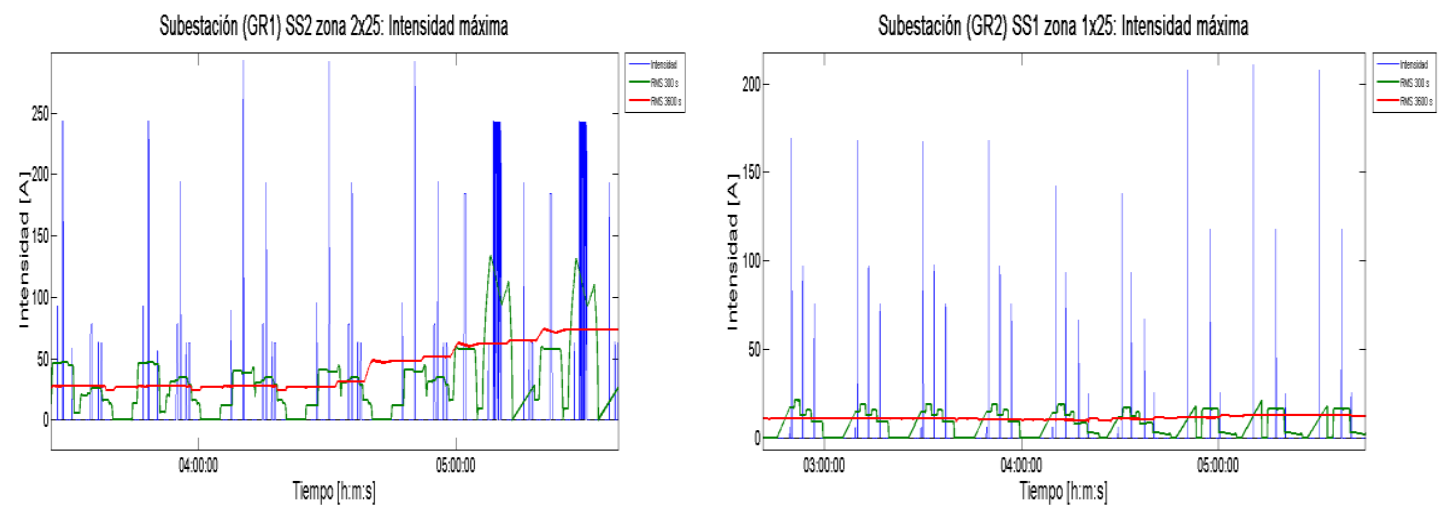

Figura 138 Intensidades máximas en a)Grupo 1 SS2 zona 2×25;b)Grupo 1 SS1 zona 2×25

El comportamiento de las subestaciones en sobrecarga para estas situaciones siguen lejos de los límites marcados por la intensidad/potencia nominal, tal y como se observa en estas gráficas y en todas las anteriores mostradas.

A pesar que tal y como se ha podido comprobar, las corrientes no se acercan a los límites, se descarta el sobredimensionamiento, ya que las tensiones mínimas, como se aprecia en la Figura 40, están bastante cerca del límite de los 19kV. 


\subsubsection{Elección 2 dimensionamiento eléctrico con 3 subestaciones de tracción y 13 autotransformadores.}

El segundo bloque significativo de resultados está representado por el siguiente ejemplo. En este se eliminan dos subestaciones de tracción, en concreto la número 2 y 4 de la zona dual del sistema eléctrico.

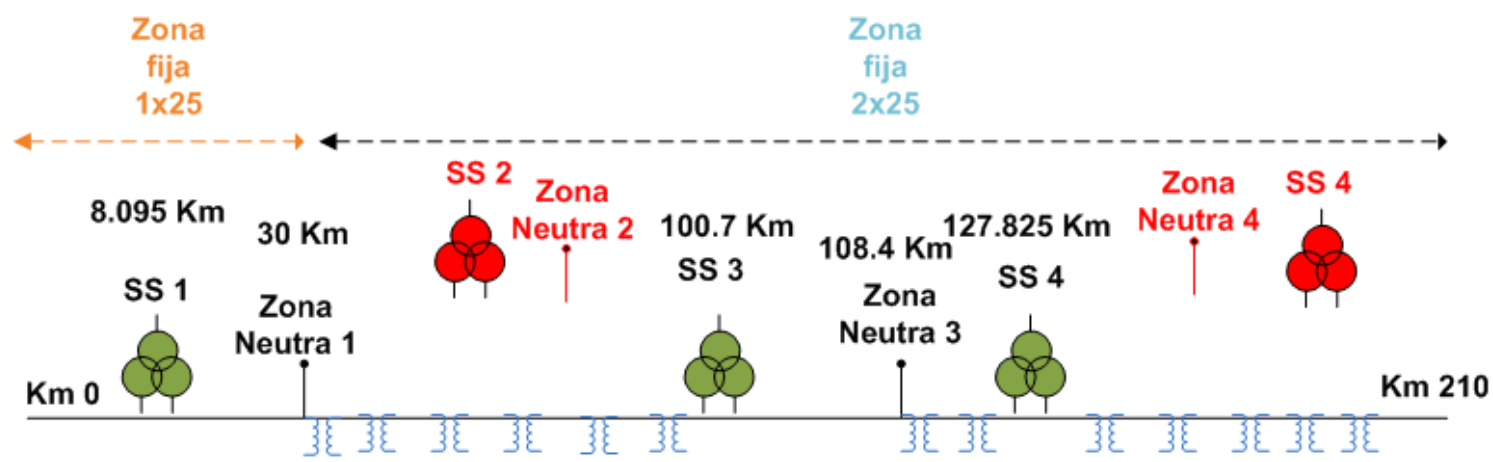

Autotransformador

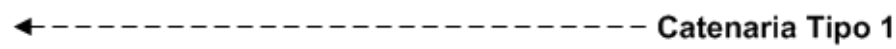

Figura 139 Ejemplo CASO 2 mejores costes instalación

Es interesante el análisis de este dimensionamiento eléctrico ya que el cambio entre este ejemplo y el anterior es muy grande, una subestación de tracción menos. Esta diferencia debería llevar mucho más al límite al sistema eléctrico, en términos de situaciones degradadas. Se van a examinar de nuevo las tensiones en catenaria y las intensidades de las subestaciones de tracción.

De nuevo el algoritmo ha esquivado las zonas con más peso, las medioambientales, y en esas zonas críticas no hay ninguna subestación ni, en este caso, autotransformador. Además de eliminar una subestación, el hecho fundamental para el ahorro de costes ha sido el provocado por la evitación de zonas muy críticas. Cabe señalar que por los pesos asociados en este ejemplo, al ser tan elevados, las pérdidas en la línea tienen una importancia relativamente pequeña. El ingeniero debería conocer cuáles son sus objetivos primordiales y si uno de ellos son las pérdidas, debería equiparar los costes/pesos de elementos/zonas a los valores normales de pérdidas debido a las corrientes por la catenaria. 


\section{- Funcionamiento normal}

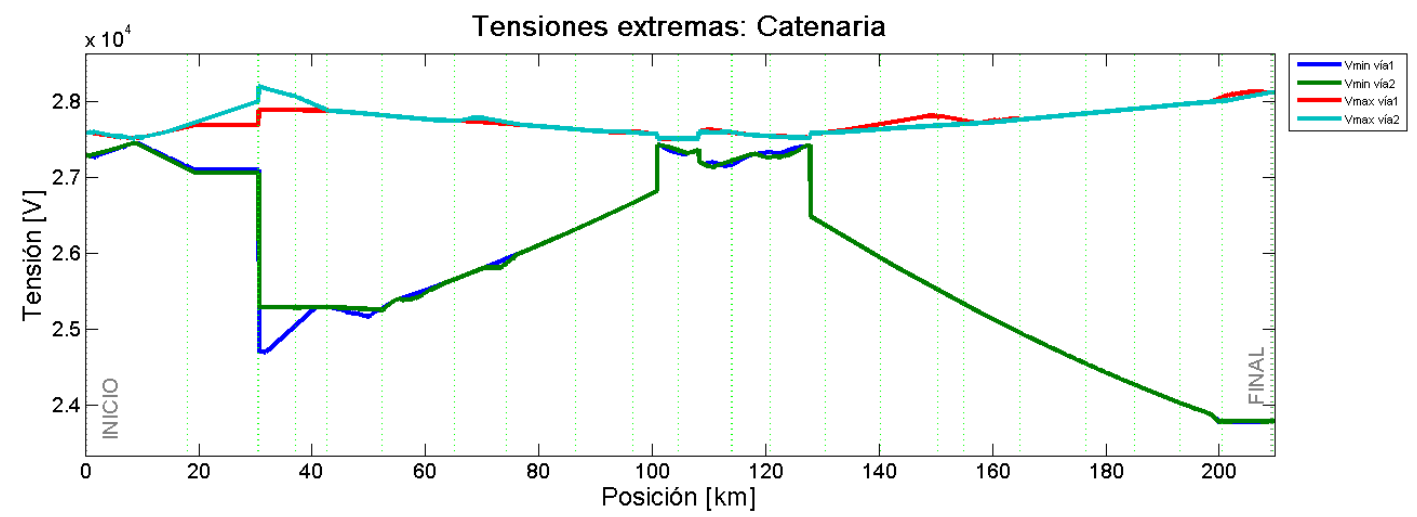

Figura 140 Tensiones extremas catenaria funcionamiento normal

El funcionamiento normal del dimensionamiento escogido define la mayor caída de tensión a partir de la tercera subestación, ya que debe alimentar hasta el final de la línea. De todos modos el límite lo marcan los aproximadamente 24000 V de tensión mínima para el caso normal.

\section{- Situaciones degradada simple, o caída de un grupo en la subestación}

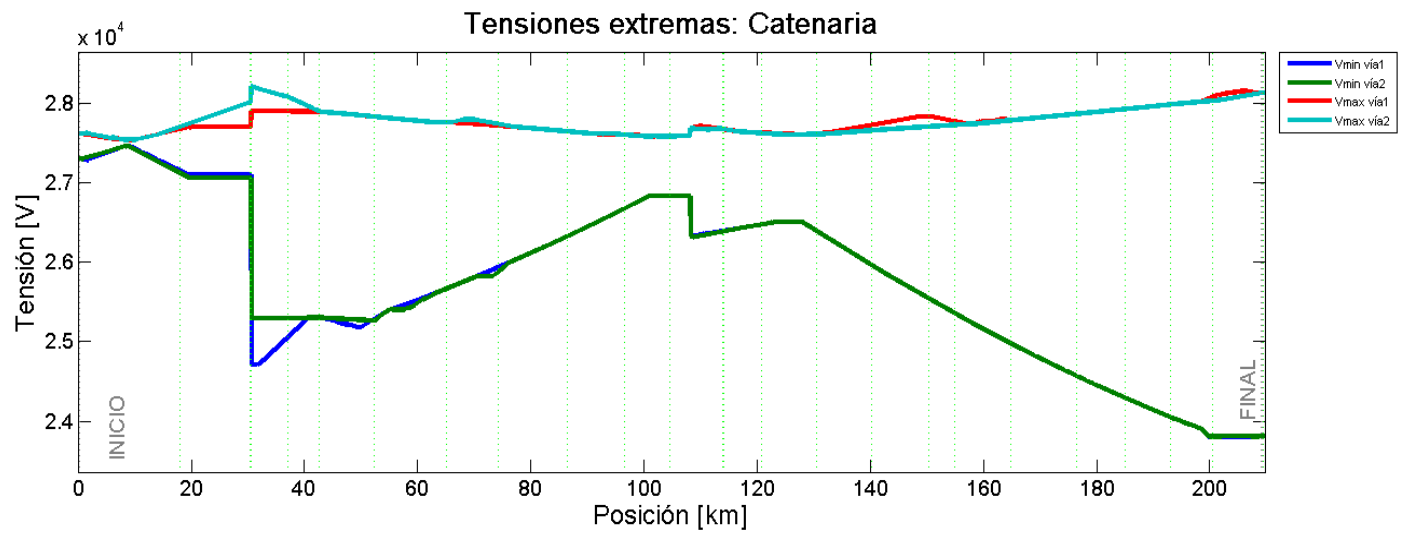

Figura 141 Degradada simple ejemplo 2 alterna dual $2 \times 25$

A pesar de la caída de un transformador de tensión, y como consecuencia la potencia suministrada por las subestaciones pasa a la mitad, la caída de tensión no es muy considerable, quedándose el límite por los $23500 \mathrm{~V}$. Cabe destacar que esta reducción del diseño del sistema eléctrico se debe fundamentalmente a que el plan de explotación no es muy exigente. Según los cálculos del ciclo de demanda máxima de potencia, se situaría sobre los $47 \mathrm{MW}$ de potencia, como se resalta, el momento donde los trenes exigen más potencia, por lo que todas estas pruebas sobre este diseño de sistema eléctrico llegan a tener una base consistente, en términos de adecuación a la demanda. 

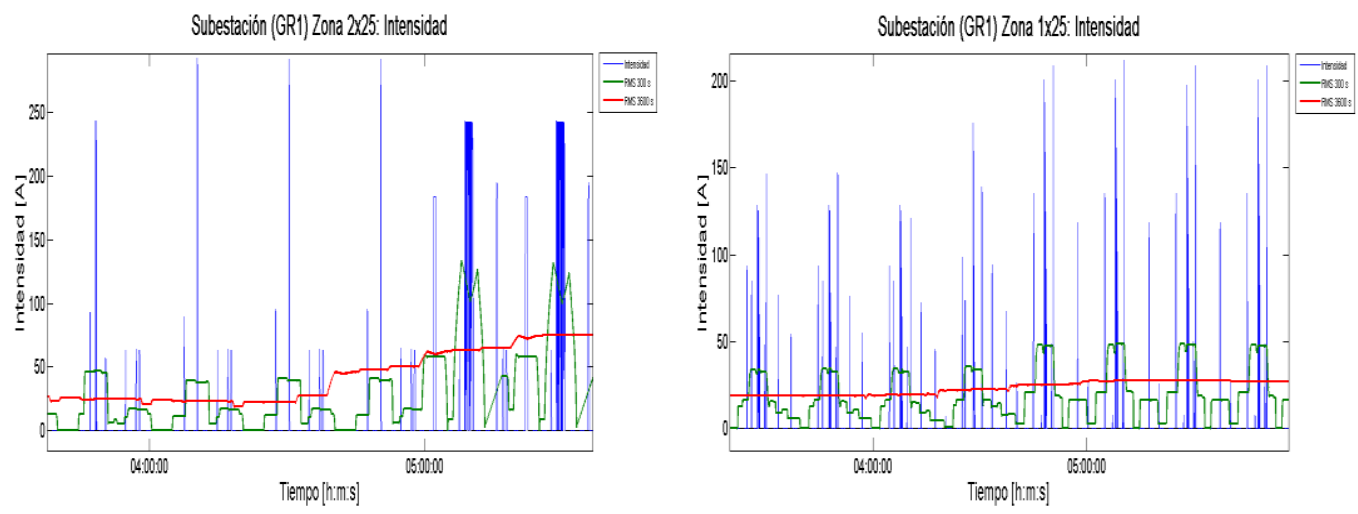

Figura 142 Intensidad máxima en las subestaciones. a) grupo 1 zona $2 \times 25$;b) grupo 1 zona $1 \times 25$

Los valores obtenidos de las intensidades en los transformadores de las subestaciones, en este caso se han elegido dos grupos de subestaciones distintas, indican que las corrientes están muy por debajo de una posible sobrecarga de las mismas, que se situaría sobre los $400 \mathrm{~V}$ :

$$
\frac{20 M W}{25000}=800 \mathrm{~A}
$$

En este caso, se sitúan los valores máximos alrededor de los 250 A

\section{- Situación degradada doble, caída subestación zona mono 1x25}

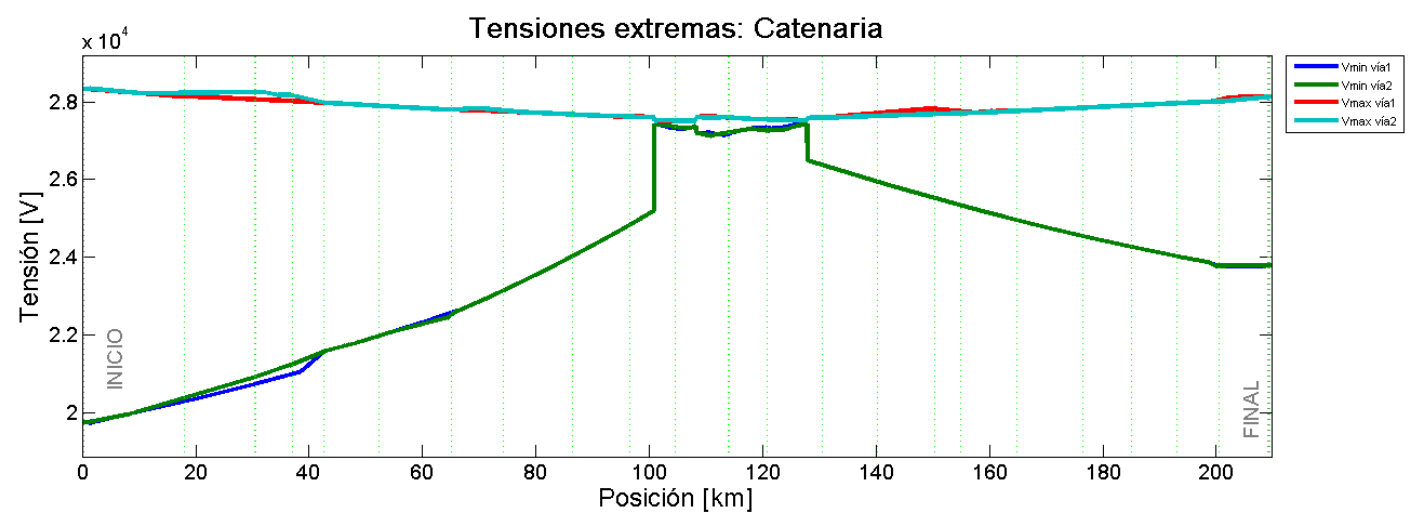

Figura 143 Tensiones extremas catenaria fallo subestación zona 1x25

Las tensiones en este caso se sitúan muy cercanas al límite de los $19000 \mathrm{~V}$ debido al fallo simulado de la subestación de la zona de $1 \times 25$. Se tiene que alimentar todo desde la subestación 3 de la zona de $2 \times 25$ y esto provoca, como es predecible una situación muy complicada, aunque según los resultados no sobrepasa los límites.

De nuevo, las intensidades en las subestaciones no superan los límites para tener que examinar las curvas de carga: 

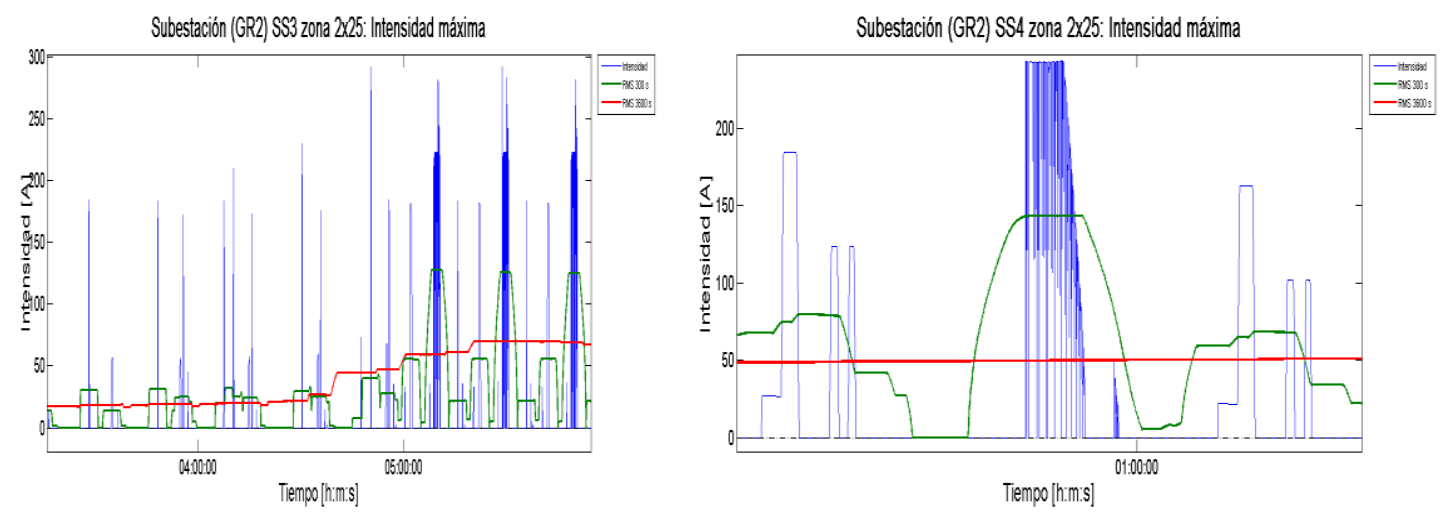

Figura 144 Intensidades Máximas en a) Grupo 2 SS3;b) Grupo 2 SS4

- Situación degradada doble, caída subestación 3 zona dual 2x25

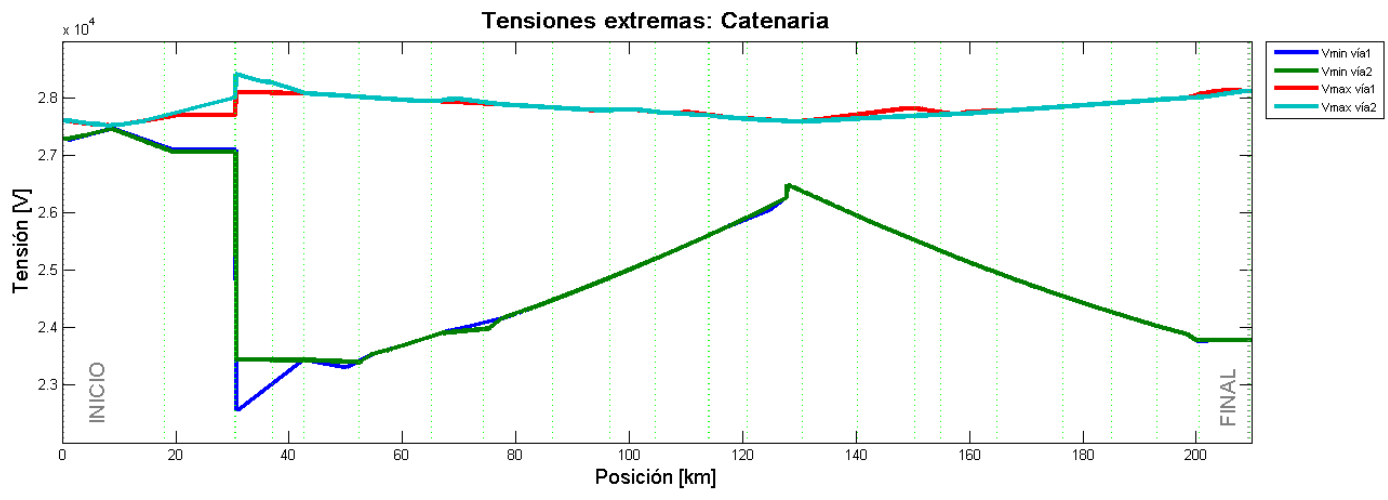

Figura 145 Tensiones extremas fallo subestación SS3 en ejemplo 2
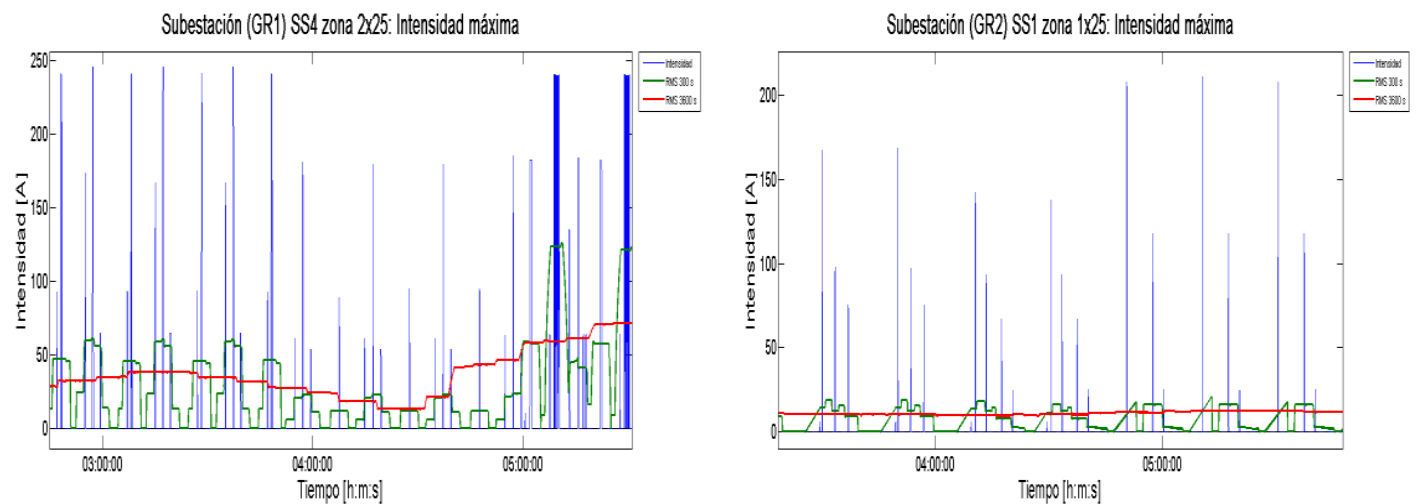

Figura 146 Intensidad máxima en a) Grupo 1 SS4; b) Grupo 2 SS1 zona 1×25

No cambia lo fundamental, que no hay peligro de sobrepasar los valores límites. En las tensiones extremas, siempre está por encima, en este caso, con la subestación restante de la zona 2×25 más la aportación de los autotransformadores que se han situado a lo largo del trayecto, son capaces de mantener la tensión hasta los $22800 \mathrm{~V}$ de máxima caída. 
En cuanto a las intensidades, de nuevo en este caso, los grupos aguantan bien las acometidas que se demandan por el plan de explotación, situándose sobre los valores de 250 A, en sus valores máximos.

- Situación degradada doble, caída subestación 4 zona dual 2x25

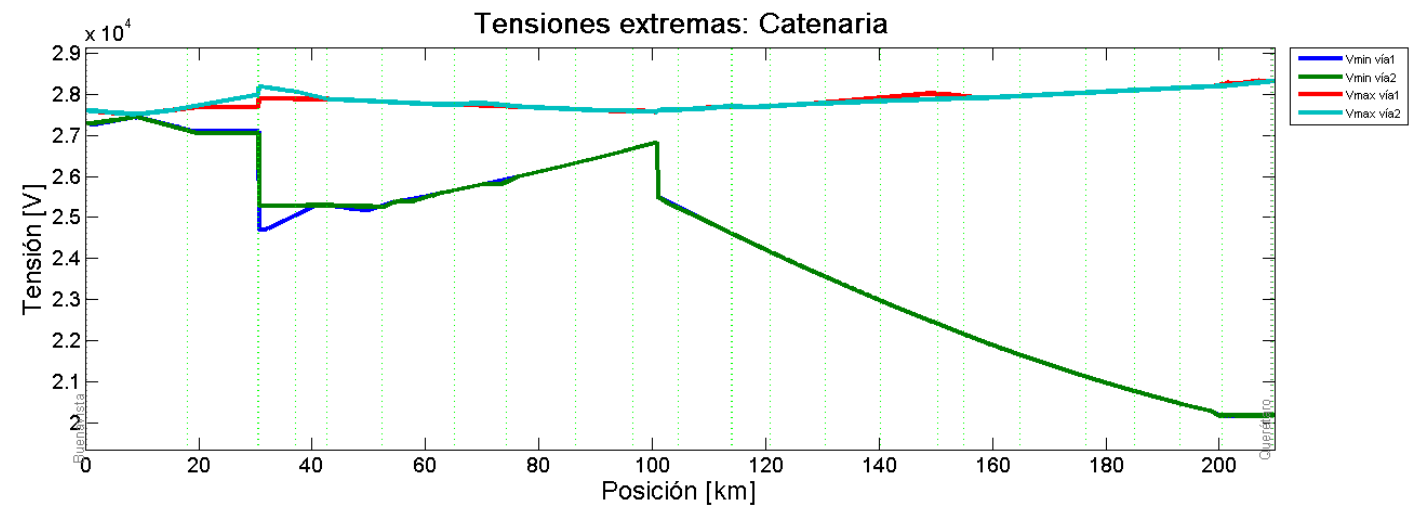

Figura 147 Tensiones extremas situación fallo en Subestación SS3 zona 2×25
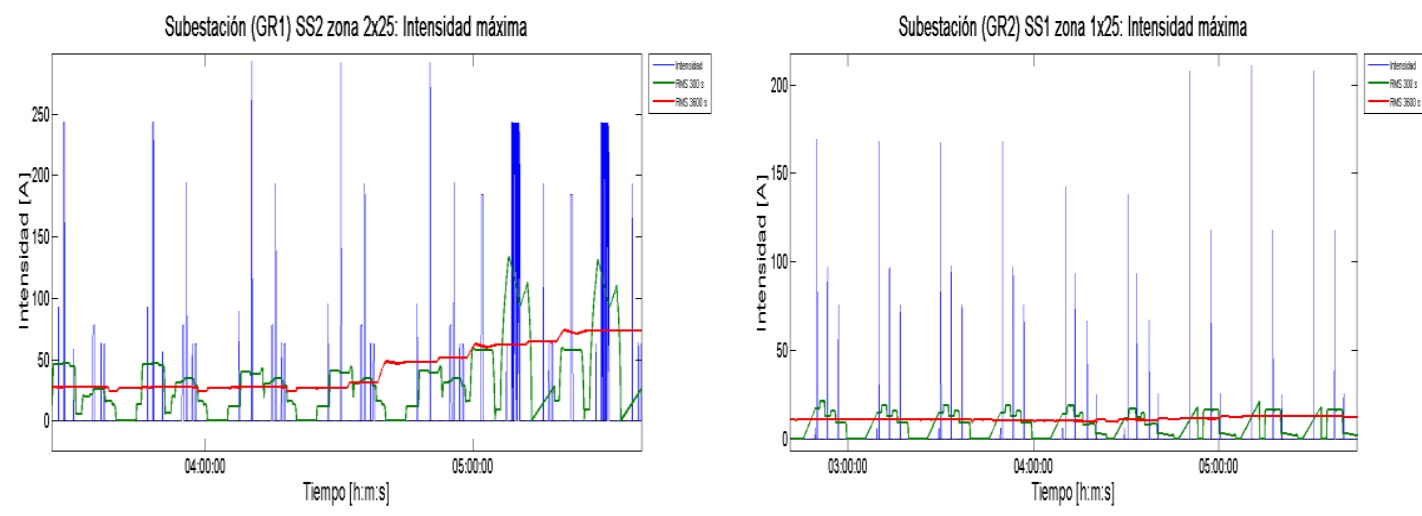

Figura 148 Intensidades máximas en a) Grupo 1 de SS2 zona 2×25;b) Grupo 1 zona 1×25

Con la simulación de un fallo en la subestación 3 de la zona 2x25, los resultados eléctricos son muy parecidos. La caída de tensión es un poco mayor que en el caso anterior y las intensidades en subestación también adquieren unos valores un poco más elevados. Pero en ningún caso se acercan al límite permitido para estos dos parámetros estudiados.

\subsubsection{Comparación entre ambos ejemplos y conclusiones.}

Los resultados de ambos dimensionamientos eléctricos han sido validados por las pruebas realizadas, tanto para caídas de tensiones mínimas y máximas en catenaria, como comprobando los niveles de intensidades máximas que soportan los transformadores de las subestaciones.

En toda validación es muy importante comprobar el comportamiento del sistema ante posibles situaciones de fallo, ya sea de un solo transformador (suponiendo más de un grupo en cada subestación), como fallo doble de la subestación. Para nuestra Metodología que evalúa durante el proceso de optimización estas posibilidades, era muy 
importante ver que el algoritmo era capaz de mejorar y ajustar los dimensionamientos eléctricos iniciales, de forma que sin menoscabar la capacidad del sistema, se pudiera ahorrar en costes, tanto de instalación como de operación. Para los ejemplos elegidos se han preparado de forma que uno tuviera un ahorro de costes más moderado y otro, el óptimo según el proceso de optimización y según los resultados recogidos de los algoritmos genéticos, el ahorro mayor posible, quitando hasta dos subestaciones.

La primera opción no tiene mayores complicaciones. Solo cuando falla la subestación última de la zona dual, se acerca a los $20 \mathrm{kV}$ de tensión mínima en la cabecera de la línea. La otra opción, la que debería ser más compleja validar, también se ha mantenido dentro de los márgenes, tanto en tensión en catenaria como intensidades máximas en subestaciones. Era de prever que a pesar de instalar un autotransformador más, la caída de tensión sería mayor que para el otro caso. Dos situaciones de fallo completo de las subestaciones dejan el límite cerca de los 19kV. Cuando falla la subestación de la zona 1x25, como en el caso de fallo de la segunda subestación de la zona $2 \times 25$ sucede esto.

Evidentemente este sistema experto o Metodología de optimización, pretende dar a conocer al ingeniero las posibilidades que puede tener para mejorar el sistema eléctrico con el que está trabajando. En función de los resultados, será él quien decida qué conviene realizar, y qué instalación debe llevar a cabo para cumplir con los requisitos del Proyecto Ferroviario. 


\subsection{Conclusiones}

Los sistemas eléctricos para ferrocarril que trabajan con corriente alterna, manejan otro tipo de factores diferentes a los que se han trabajado en el Capítulo 5, para corriente continua. La diferenciación entre ambos sistemas, a nivel de decisión de diseño de alto nivel, se ha incluido en la Metodología de esta Tesis, tal y como se ha descrito a lo largo de este Capítulo.

Conociendo que los proyectos de este tipo de electrificación, suelen trabajar tanto en sistemas monotensión ó 1x25, como en bitensión ó $2 \times 25$, se ha diferenciado la búsqueda del óptimo, para poder analizar mejor el comportamiento del optimizador. Para ambos casos se ha conseguido trabajar con escenarios aptos para el cometido de pruebas, con los que el diseñador puede evaluar perfectamente hacia dónde llegar con el sistema eléctrico, tanto a nivel de limitaciones eléctricas, así como comprobar el soporte de energía que proporciona a la explotación ferroviaria y también en cuanto a costes directos e indirectos. Focalizando en el desarrollo de las pruebas, y en los valores devueltos por los 3 algoritmos genéticos, se observa que a diferencia de los tests realizados en el Capítulo 5, en este caso, al tener una mayor flexibilidad para modificar el diseño inicial, los algoritmos proveen resultados finales con mucha más diversidad en el espacio de soluciones. La comparativa entre los 3 algoritmos, de nuevo dicta que el algoritmo NSGA-II necesita mucho más tiempo para obtener resultados, que en definitiva son muy parejos en valores de fitness respecto a los resultados de los otros dos algoritmos.

En cuanto a las verificaciones de los baremos eléctricos de los diseños elegidos, se muestra que éstos son robustos a las normativas planteadas tanto a nivel de caídas de tensión, intensidades en conductores como sobrecalentamientos de los transformadores de tensión de las subestaciones. Verificando de esta manera que el optimizador ha filtrado los diseños no aptos. Esto sumado a que efectivamente tratan de evitar las zonas con más peso, ya sea medioambiental, de conexión con la red de distribución o de mantenimiento, hace que las conclusiones sean muy positivas de cara a futuros trabajos con la Metodología. 


\section{Bibliografía}

[1] L. Batistelli, M. Pagano y D. Proto, «Short Circuit Modelling and Simulation of $2 \times 25$ kV High Speed Railways,» de Second Asia International Conference on Modelling \& Simulation, 2008.

[2] T. Kneschke, «Simple Method for Determination of Substation Spacing for AC and DC electrification systems, " IEEE Transactions on industry applications, Vols. \%1 de \%2IA-22, no 20, pp. 763-780, 1986.

[3] E. Pilo, L. Rouco y A. Fernández, «A reduced representation of $2 \times 25 \mathrm{kV}$ electrical,» de Proceedings of the IEEE/ASME Joint Rail Conference, Chicago, Illinois, 2003.

[4] E. Pilo, «Diseño óptimo de la electrificación de ferrocarriles de alta velocidad,» Madrid, 2003.

[5] E. Pilo, L. Rouco y E. Fernández, "Catenary and Autotransformer coupled optimization for 2x25kV systems planning," de Witt Transactions on the Built Environment, 2006.

[6] E. Pilo, L. Rouco y A. Fernández, "An Optimization Procedure to Determine the Topology of AC Railways Power Supply Networks," de ASME/IEEE 2007 Joint Rail Conference and Internal Combustion Engine Division, Pueblo, Colorado, 2007.

[7] J. R. Jiménez-Octavio y E. Pilo, "Optimal design of power supply systems using genetic algorithms,» Power Supply, Energy Management and Catenary Problems, p. 135, 2010.

[8] T.-H. Chen y Y.-F. Hsu, «Systematized short-circuit analysis of a $2 \times 25 \mathrm{kV}$ electric traction network, " Electric Power Systems Research, vol. 47, no 2, pp. 133-142, 1998.

[9] H. Zhengqing, Z. Yuge, L. Shuping y G. Shibin, «Modeling and Simulation for Traction Power Supply System of High-Speed Railway, " de Power and Energy Engineering Conference (APPEEC), Asia-Pacific, 2011.

[10] R. Hill y I. J. Cevik, "Parallel computer simulation of autotransformer-fed AC traction networks,» de Railroad Conference, Technical Papers Presented at the 1990 ASME/IEEE Joint, 1990.

[11] P. C. Tan, P. C. Loh y D. G. Holmes, «A robust multilevel hybrid compensation system for 25-kV electrified railway applications," IEEE Transactions on Power Electronics, vol. 19, no 4, pp. 1043-1052, 2004.

[12] H. M. Lee, G. D. Kim, S. C. Oh y S. H. Park, «Optimal Location and design of Rc-Bank on Korean Electric Railway System,» pp. 118-128, 2005.

[13] T. K. Ho, Y. L. Chi, J. Wang y K. K. Lueng, «Probabilistic load flow in AC electrified railways,» 
IEE Proceedings Electric Power Applications, vol. 152, no 4, pp. 1003-1013, 2005.

[14] S. H. Lee, J.-O. Kim y H. S. Jung, «Analysis of catenary voltage of an AT-fed AC HSR system,» IEEE Transactions on Vehicular Technology, vol. 53, no 6, pp. 1856-1862, 2004.

[15] T. Kohonen, «An introduction to neural computing,» Neural networks 1.1, pp. 3-16, 1988.

[16] L. Abrahamsson y L. Söder, «Railway power supply investment decisions considering the voltage drops - Assuming the future traffic to be known," de ISAP in Proceedings 15th IEEE International Conference, 2009.

[17] M. Olofsson, G. Andersson y L. Söder, «Optimal operation of the Swedish railway system,» de Proceedings International Conference Elect. Railways United Europe, 1995.

[18] L. Abrahamsson, S. Östlund y L. Söder, «Optimal PowerFlow (OPF) Model with Unified ACDC Load Flow and Optimal Commitmentfor an AC-catenary Railway Power Supply System (RPSS) fed by aHigh Voltage DC (HVDC) transmission line,» IEEE Press, 2012.

[19] E. R. Agency, "Set of Specifications 2 (ETCS baseline 3 and GSM-R Baserailway, European Railway Agency (ERA))," 2013, Valenciennes. [En línea]. Available:

http://www.era.europa.eu/Core-Activities/ERTMS/Pages/Set-of-specifications-2.aspx.

[20] R. J. Hill, D. C. Carpenter y T. Tasar, «Railway track admittance, earth-leakage effects and track circuit operation,» de Proceedings Technical Papers Presented at the 1989 IEEE/ASME Joint. IEEE, 1989, 1989.

[21] A. Mariscotti, «Distribution of the traction return current in AC and DC electric railway systems, " IEEE Transactions on Power Delivery, vol. 18, no 4, pp. 1422-1432, 2003.

[22] Z. D. Alan y T. Barlo, "Stray Current Corrosion in Electrified Rail Systems -- Final Report,» Infrastructure Technology Institute, Northwestern University, Chicago, 1995.

[23] M. Niasati y A. Gholami, "Overview of stray current control in DC railway systems,» de International Conference on ICRE 2008, 2008. 


\section{Conclusiones. Aportaciones y planteamiento futuro de mejora. Publicaciones}

El diseño del sistema eléctrico del sistema ferroviario es una tarea compleja y con muchos factores involucrados. Aplicando los conocimientos adquiridos en validación de sistema eléctrico y a partir de investigaciones referentes al tema, el principal objetivo de esta Tesis estaba enmarcado en el desarrollo de una Metodología dirigida a la optimización de los diseños de los sistemas eléctricos ferroviarios.

Para los objetivos marcados en esta Tesis se consideró que se debían tener en cuenta los elementos tradicionales en la toma de decisiones, a la hora de trabajar con un proyecto de diseño de sistema eléctrico o dimensionamiento del mismo. La solución aportada se ha trasladado tanto a los sistemas ferroviarios que trabajan con corriente continua, como a los de corriente alterna, tanto en $1 \times 25$ como en $2 \times 25$.

El Capítulo 1 se introduce al lector en el muestreo de los diversos sistemas de energía que se han ido aplicando al movimiento sobre raíles. La evolución desde las primeras máquinas de vapor hasta la predominancia de nuestros días de la electricidad aplicada, tanto a sistemas ferroviarios para distancias cortas, como para largos recorridos de trenes de alta velocidad.

La intención del Capítulo 2 es situar al lector en aspectos básicos que se van a ser fundamentales en la aplicación de la Metodología. Se divide en tres apartados donde el primero tiene como principal meta el describir el por qué de la diferenciación entre los distintos sistemas eléctricos de tracción y cuáles son sus características más relevantes. El segundo subapartado se centra en enumerar los principales elementos que están involucrados en la Metodología, sobre todo los pertenecientes a los críticos para los sistemas eléctricos. Finalmente el tercero habla de la importancia de investigar acerca de las consecuencias en temas como mantenimiento, instalación, impacto medioambiental y conexión con la red general, en función del diseño final ferroviario, y en concreto del sistema eléctrico.

El Capítulo 3 se ha dedicado a explicar los detalles principales del simulador Hamlet.

En el Capítulo 4 se explica la Metodología completa, realizando un repaso a las técnicas de optimización. Se detallan los algoritmos genéticos elegidos. La adaptación del modelo al problema que se está tratando, la focalización del sistema eléctrico y el rango de construcción para la solución permitida por la Metodología, también se explican en este Capítulo.

Finalmente en los Capítulos 5 y 6 se exponen diversos casos de prueba, tanto para modelos de corriente continua, como para sistemas en alterna. En ambos capítulos se ha tratado de exponer casos reales de sistemas ferroviarios donde se planteó en su momento el problema de validación del sistema eléctrico, y donde la propuesta aportada 
por el sistema experto llegó a ser satisfactoria y tenida en cuenta para la toma de decisión final.

\subsection{Aportaciones obtenidas}

A lo largo de la Tesis se ha desgranado los trabajos realizados, investigaciones llevadas a cabo y resultados de las pruebas más significativas. Uno de estos trabajos importantes ha sido el desarrollo de un simulador completo, Hamlet, que ha permitido eficazmente contrastar tanto las simulaciones de los sistemas eléctricos, como trabajar con las optimizaciones. Así como el desarrollo del simulador existen otras tareas que se han ido realizando paralelas a aquellas que se han pensado son aportaciones obtenidas, de modo que pueden ser empleadas en nuevas investigaciones acerca de la optimización de diseños de sistemas eléctricos ferroviarios. Estas serían:

1. Implantación y comparación de resultados de tres algoritmos genéticos en el proceso de optimización.

2. Aplicación directa a sistemas de electrificación

3. Ponderación y discretización zonal

4. Aplicación directa con el Pico de Demanda Máxima de Potencia

5. Obtención de resultados aplicando Tests de Convergencia para Restricciones aplicadas en el modelo de optimización

\subsubsection{Implantación de Algoritmos genéticos en el proceso de optimización}

La múltiple variabilidad de los sistemas ferroviarios, y sobre todo el planteamiento inicial de que la optimización tuviera en cuenta la mayor parte de los componentes de estos sistemas, ha hecho que nos decantáramos por un sistema de optimización que permitiera ante todo, flexibilidad de estudio del modelo.

En un paso previo se analizaron todas las posibilidades posibles de integrar un algoritmo de optimización. Finalmente se concluyó que lo mejor era adaptar algoritmos genéticos. Además permitía trabajar la dicotomía planteada en el análisis de resultados al tener por una parte costes de instalación y por otra parte costes de operación, que en principio son contrapuestos.

Se implantaron tres algoritmos genéticos de última generación y con buenos resultados en términos de complejidad temporal.

- Épsilon-MOEA

- AMGA-II

- NSGA-II

Los tres han sido adaptados e implementados para que pudieran interactuar con el simulador, más concretamente con la parte eléctrica, y también con el sistema de funciones objetivo y restricciones del modelo, que obviamente vienen acompañados de este tipo de técnicas. 


\subsubsection{Aplicación directa a sistemas de electrificación}

Tal y como se ha comentado anteriormente, todo ha ido encaminado a que la Metodología se pudiera aplicar a cualquier tipo de sistema de electrificación que en esos momentos hay implantados en las líneas ferroviarias. Las pruebas realizadas y mostradas en diversos casos y de diversa complejidad a sistemas de corriente continua, y corriente alterna, tanto en modo simple 1x25, como en $2 \times 25$, e incluso sistema mixto, así lo demuestran.

\subsubsection{Ponderación y Discretización Zonal}

Al escoger técnicas de optimización basadas en algoritmos genéticos, definió un modo de modelar el sistema de costes para que fuera adaptable a las funciones objetivo y restricciones que serían evaluadas por los algoritmos.

El sistema de ponderación es flexible para que el ingeniero pueda evaluar si poner costes directos de los elementos involucrados en el sistema eléctrico, o bien indirectos o mejor llamados estimados. Según la experiencia de proyectos anteriores o calidad de cada elemento, el ingeniero será capaz de asignar valoraciones a los elementos de forma que ese valor sea constitutivo de complementar una estabilidad respecto al conjunto de elementos tenidos en cuenta en el sistema. Es decir si se evalúa un elemento muy por encima de los demás, eso significaría que hay un valor muy fuera de la mediana de valores, por lo que el sistema, en la medida en que se pueda evitar, va a tratar de eliminarlo de la inclusión en los resultados optimizados. Y por supuesto al revés.

La posibilidad de poder combinar subestaciones con distinto grupo de transformadores/rectificadores, es otra de las inclusiones que se pueden añadir como aportaciones. Es interesante porque a pesar de que añaden más genes al genotipo, y por tanto más complejo temporalmente se convierte, el resultado final es más completo.

Se desarrolló también la implicación de zonas específicas de impacto, recorridas por el trayecto proyectado de la línea ferroviaria:

- Zonas de alto valor medioambiental

- Zonas con dificultad para conexión con la red general eléctrica

- Zonas de acceso y mantenimiento complicado del sistema eléctrico

- Zonas de instalación de los elementos eléctricos básicos.

La discretización divide el espacio general entre las zonas reales de impacto, y al dotar de valor a cada una de ellas, se le aplica un valor que deberán pagar cada vez que se quiera incluir un elemento eléctrico básico en esa zona. Muy importante para determinar el posicionamiento de las subestaciones, zonas neutras $y$ autotransformadores. 


\subsubsection{Aplicación directa del Pico de Demanda Máxima de Potencia.}

Para la aplicación directa de un sistema que optimice un sistema eléctrico completo de un sistema ferroviario, es necesario acudir a un tipo de modelo que consiga focalizar en la mayor medida posible, la capacidad y características del modelo general.

Para esta optimización se ha desarrollado y validado, un método que consiste en recoger los picos de demanda máxima de potencia de los trenes en circulación, para cada proyecto que se vaya a estudiar. De modo que primero se realiza una simulación con el sistema eléctrico inicial, el material rodante que se vaya a implantar, el sistema de señalización y por supuesto, la infraestructura sobre la que van a circular los trenes. Una vez terminada la simulación se obtienen los datos de:

- Instante de tiempo donde se ha demandado más potencia por parte de los trenes durante toda la simulación

- Para cada subestación de tracción, instante donde se le ha demandado más potencia a cada subestación. Esto se utilizará para examinar las situaciones degradadas de servicio de las mismas

La idea es que con este ciclo de máxima demanda, que será aplicable a cualquier situación, independientemente del sistema eléctrico, se examinará el estado del sistema eléctrico para cada optimización.

Permite aplicar comportamiento local a un sistema global y complejo del sistema ferroviario.

\subsubsection{Obtención de resultados aplicando Tests de Convergencia para Restricciones aplicadas en el modelo de optimización}

Planteándose como técnica de optimización principal los algoritmos genéticos, uno de las principales tareas que se resolvió fueron los modelos de ecuaciones para las funciones objetivo y las restricciones.

Las funciones objetivo han tratado de encapsular toda la información de la configuración, teniendo como meta la minimización de costes ponderados, tanto de los elementos involucrados en cada decodificación del genotipo planteado, como en los costes asociados a las zonas donde se plantean la construcción de algún elemento básico del sistema eléctrico. Por último en la función objetivo de los costes de Operación, se analizan las pérdidas de energía que sufre cada sistema eléctrico asociado, dando así una nueva forma de evaluación automática para conocer la capacidad de cada individuo chequeado.

Las restricciones también han sido un punto importante en el trabajo, ya que permiten acotar la verdadera validez del sistema analizado. Para esta Tesis se presentan restricciones al sistema eléctrico de forma que un genotipo no debería sobrepasar el límite presupuestario planificado por el Ingeniero y además se presenta un conjunto de restricciones englobadas en restricciones de tipo de convergencia eléctrica donde: 
- Convergencia del sistema eléctrico con la configuración en funcionamiento normal.

- Convergencia del sistema eléctrico en situación de fallo simple de las subestaciones de tracción.

- Convergencia del sistema eléctrico en situación de fallo completo de las subestaciones de tracción.

- Exámenes de ciclos de demanda de potencia global del sistema y a nivel de subestaciones afectadas y adyacentes.

- Exámenes de límites de tensión

- En contacto catenaria-pantógrafo o tercer carril-patín

- En tensiones de retorno carril-tierra

Todo esto devuelve una penalización creciente dependiendo de la gravedad de la violación de la restricción. Este método permite manejar las poblaciones en cada generación de modo que las características mejores se puedan perpetuar, aunque sean en fallo, ya que es probable que en algún momento el sistema eléctrico planteado no tenga solución factible y se tenga que optar por la mejor posible, para a posteriori poder mejorarla en los puntos donde se produzca el fallo más crítico.

\subsection{Planteamiento futuro de la investigación}

En los últimos pasos antes de la presentación de la memoria de la Tesis Doctoral, siempre surgen dudas acerca de la profundidad de la misma. A lo largo del trabajo de investigación, desarrollo y pruebas se va acotando el trabajo a realizar, dejando para más adelante otros que se consideran también interesantes o que en ese momento, no se tenía claro como encajar dentro del mecanismo creado para el acoplamiento de las piezas que hasta el momento se tenían dispuestas. En este apartado se van a proponer algunas de las ideas que no se han puesto en práctica por el momento, pero que se consideran bastante oportunas e interesantes para mejorar la Metodología.

\subsubsection{Incorporar algoritmo genético de última generación}

Los algoritmos incorporados como ya se ha dicho en repetidas ocasiones, tienen una buena complejidad temporal y tienen una funcionalidad bastante aceptable. La búsqueda del conjunto se pensó para obtener una perspectiva de comparación bastante buena. Otra perspectiva la daría el poder adaptar al método de optimización un algoritmo multiobjetivo de modo que se mejorase la complejidad temporal y de esta manera poder encontrar respuestas en un tiempo menor.

\subsubsection{Elementos estadísticos en la aplicación de análisis del frente de Pareto}

Una buena aportación sería incorporar en el tratamiento de resultados algún tipo de análisis estadístico, de modo que se pudiera encontrar un patrón de calidad del sistema eléctrico en función de los individuos de la población de la generación última, y en términos estadísticos, llegar a conclusiones respecto a la configuración inicial de los parámetros de ponderación y discretización zonal, contrapuestos con los resultados 
finales, no solo del Frente de Pareto, sino yendo más allá, en ránkings menores de fitness de las poblaciones.

\subsubsection{Proyecciones en las valoraciones de los elementos eléctricos}

En el caso de una subestación, no tan solo es importante el valor directo que se le pueda asignar, ya sea el real o el ponderado. El planteamiento es conocer por ejemplo otros puntos de vista de calidad de coste de estos elementos. Por ejemplo, conseguir el ratio vida estimada versus gasto energético $\mathrm{kWh}$. La valoración de, en este caso la subestación, sería más completa. Esto trasladado a todos los elementos involucrados proporcionaría un resultado final muy adecuado a las necesidades no tan solo presentes, sino a futuro, vida de duración de los elementos eléctricos.

\subsubsection{Combinación con sistemas de regeneración de energía}

La regeneración de energía está cada vez más en disposición de ser incorporada a los sistemas ferroviarios. Permite aprovechar mejor la energía, ya sea distribuyendo a los trenes adyacentes en fase de tracción la energía generada en sistemas de frenado regenerativo, o bien inyectándolas directamente en la catenaria, o por último cargando acumuladores, o bien a bordo de los trenes o posicionados a lo largo de la línea.

Este supuesto tiene mayor complejidad ya que implica tener en cuenta aspectos que no son fijos, tal y como si se pueden obtener usando el criterio de pico de demanda máxima de potencia. Requerirá un cambio en la filosofía fundamental de la herramienta pero se tendrá que analizar si el beneficio final es consecuente con el esfuerzo.

\subsubsection{Combinación con cálculos de optimización de consumo de energía}

En sintonía con el punto anterior, y teniendo en cuenta la multitud de estudios para obtener mejoras en el consumo de energía de los sistemas ferroviarios, el poder combinar ambos métodos de optimización, para que el resultado final tuviera en cuenta el consumo obtenido y como mejorarlo en función del sistema diseñado, se habría alcanzado un punto en el que el resultado o resultados finales, darían al ingeniero un sistema eléctrico muy terminado, muy complejo y muy optimizado en términos de ahorro presupuestario y energético. 


\subsection{Publicaciones y visibilidad investigadora}

El desarrollo de una Tesis Doctoral implica un trabajo arduo de investigación durante todo el proceso que conlleva el mismo. Incluso después de la finalización de la Memoria, también debería proseguir el autor con esa tarea investigadora que, se podría decir, comienza con la Tesis Doctoral. En nuestro caso, a lo largo de los años se ha podido trabajar en diversos ámbitos, todos relacionados con tareas de optimización de procesos ferroviarios, confluyendo en un área de vital importancia tanto a nivel operacional como presupuestario, como es el sistema eléctrico de las líneas ferroviarias. En este camino que se enmarcaría en el contexto de $\mathrm{I}+\mathrm{D}+\mathrm{i}$, se ha colaborado activamente en la consecución de diversos trabajos y publicaciones. A continuación se muestran los eventos más relevantes que se han conseguido a lo largo de este camino.

- Artículos en Revistas Científicas de Impacto englobadas en JCR

- M. Soler, J, López, JM Mera, J. Maroto. (2015) "Methodology for Multiobjective Optimization of the AC Railway Power Supply System”. IEEE Transactions on Intelligent Transportation Systems. Pags. 1-12, Vol: PP, Issue: 99. Mayo 2015.

Factor de impacto JCR: 2.377 Q1

- M. Soler, J. López, JM. Mera, S. Tapia. "Expert system using Multiobjective optimization of the Direct Current railway power supply system". Transport Taylor and Francis. Aceptado para publicación en mayo de 2015. En Producción hasta Octubre de 2015.

Factor de impacto JCR: 0.553 Q3

- E. Rodrigo, S. Tapia, JM. Mera, M.Soler.(2013) "Optimizing Electric Rail Energy Consumption using the Lagrange Multiplier Technique”. Journal of Transportation Engineering. Vol: 139, Issue: 3. Pags. 321-329. Marzo 2013.

Factor de impacto JCR: 0.797 Q2

- JM. Mera,E. Carabaño,M. Soler \& E. Castellote. (2014). "Increasing metro line capacity by optimisation of track circuit in a speed code Automatic Train Protection system". Proceedings of the Institution of Mechanical Engineers, Part F: Journal of Rail and Rapid Transit. Aceptada Marzo 2014. Pag. 1-14.

Factor de impacto JCR: 0.857 Q3 
- Otras publicaciones

- M. Soler, JM: Mera, J. López. (2013). "Simulation system for the optimization of a block distribution under the ERTMS-1 signalling system". COMPRAIL 2013. Pags 61-73.

- JM. Mera, E. Castellote, MA. Pérez, M.Soler. (2009). "Flexibility, modularity and interactivitiy. A new working strategy on railway line simulators". Models and technologies for intelligent transportation systems in Proceedings of the International Conference. Rome, Junio 2009.

\section{- Congresos y Conferencias}

- "Methodology of optimization design on railway high speed electric dimensioning". JR-EAST UIC Tokio 2015 Highspeed 9th World Congress on High Speed Rail. 7-10 Julio 2015 